\title{
Search for Sterile Neutrinos with
}

\section{the MINOS Long-Baseline \\ Experiment}

\author{
Ashley Michael Timmons
}

School of Physics and Astronomy

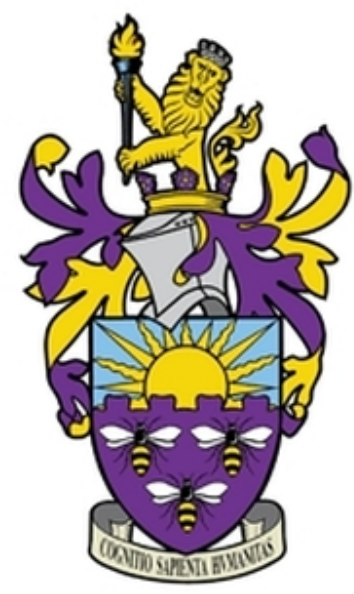

A thesis submitted to the University of Manchester for the degree of Doctor of Philosophy

in the Faculty of Science and Engineering 


\section{Contents}

$\begin{array}{lr}\text { Declaration } & 18\end{array}$

$\begin{array}{lr}\text { Copyright } & 19\end{array}$

$\begin{array}{lr}\text { Acknowledgements } & 20\end{array}$

$\begin{array}{ll}\text { Dedication } & 22\end{array}$

1 Introduction $\quad 23$

2 Neutrino Physics $\quad 26$

2.1 The History of the Neutrino . . . . . . . . . . . . . . . . 26

2.1 .1 The First Hints of a Neutrino . . . . . . . . . . . . 26

2.1.2 The Neutrino is Discovered . . . . . . . . . . . . . 28

2.1 .3 Different Neutrino Types . . . . . . . . . . . . . . . . . . 29

2.1.4 Properties of the Neutrino . . . . . . . . . . . . . . . 31

2.2 Neutrino Oscillations . . . . . . . . . . . . . . . . . . . . . 32

2.2 .1 Solar Neutrinos . . . . . . . . . . . . . . . . . 33

2.2.2 Atmospheric Neutrinos . . . . . . . . . . . . . . . 36

2.2.3 Reactor Neutrinos . . . . . . . . . . . . . . . . . . 38

2.2.4 Accelerator Neutrino Beams . . . . . . . . . . . . . . . 39

2.2.5 Oscillation Formalism _. . . . . . . . . . . . . . 40

2.2.6 Three-Flavour Model . . . . . . . . . . . . . . . . . . . 44 
2.3 Sterile Neutrinos . . . . . . . . . . . . . . . . . . . . . . . 47

2.3.1 Electron Neutrino Appearance Anomalies . . . . . . . . . 47

2.3.2 Reactor Anomalies . . . . . . . . . . . . . . . . . 49

2.3.3 Gallium Anomalies . . . . . . . . . . . . . . . . 51

2.3.4 The Sterile Neutrino Model . . . . . . . . . . . . . . . . . 52

3 The MINOS and MINOS+ Experiments 55

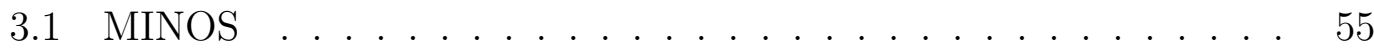

3.2 MINOS $+\ldots \ldots \ldots \ldots \ldots \ldots \ldots$

3.3 The NuMi Beam . . . . . . . . . . . . . . . . . . . . . 60

3.3.1 Slip-Stacking . . . . . . . . . . . . . . 62

3.3.2 The NuMI Target . . . . . . . . . . . . . . . . . 65

3.4 The MINOS Detectors . . . . . . . . . . . . . . . 66

3.4 .1 Overview ...................... 66

3.4.2 Two-Detector Experimental Setup . . . . . . . . . . . 67

3.5 Steel . . . . . . . . . . . . . . . . . . 70

3.6 Plastic Scintillator . . . . . . . . . . . . . . . . . 70

3.7 Photomultiplier Tubes . . . . . . . . . . . . . . . . 72

3.8 Magnetic Field . . . . . . . . . . . . . . . . . . . . 73

3.9 Electronics and Readout . . . . . . . . . . . . . . . 74

3.10 Light Injection . . . . . . . . . . . . . . . . . . 74

3.11 Triggering . . . . . . . . . . . . . . . . 75

3.12 The Calibration Detector . . . . . . . . . . . . . . 75

3.13 Neutrino Interactions in the Detectors . . . . . . . . . . 76

3.13 .1 Event Topologies . . . . . . . . . . . . . . 76

3.13 .2 Neutrino Cross Sections . . . . . . . . . . . . . 77

4 Calibration and Reconstruction $\quad 80$

4.1 Calibration ...................... . . 80 
4.1.1 Linearity Calibration . . . . . . . . . . . . . . . 81

4.1.2 Gain and Drift Calibration . . . . . . . . . . . . . . 82

4.1.3 Strip-to-Strip Non-Uniformity Calibration . . . . . . . . . 83

4.1.4 WLS Fibre Attenuation Correction . . . . . . . . . . . 84

4.1.5 Inter-Detector Calibration . . . . . . . . . . . . 84

4.1.6 Summary of Calibration Chain . . . . . . . . . 87

4.1.7 Absolute Energy Scale . . . . . . . . . . . . . . . 88

4.2 Simulation Software . . . . . . . . . . . . . . . . 89

4.2.1 Simulating the NuMI Beam . . . . . . . . . . . . 90

4.2.2 Simulating the MINOS Detectors . . . . . . . . . . . 92

4.3 Reconstruction ......................... 94

4.3.1 Track Reconstruction . . . . . . . . . . . . . . 95

4.3.2 Shower Reconstruction . . . . . . . . . . . . . . 97

4.3.3 Event Building . . . . . . . . . . . . . . . . . . 97

4.4 Shower Energy Estimator _. . . . . . . . . . . . . . . . . . . 98

4.4 .1 The $k \mathrm{NN}$ Algorithm . . . . . . . . . . . . . . . . . . . 98

4.4.2 Shower Energy Estimator Uncertainties . . . . . . . . . . . 99

5 Event Selection $\quad 102$

5.1 Preselection . . . . . . . . . . . . . . . . . . 102

5.2 NC Selection . . . . . . . . . . . . . . . . 107

5.2 .1 NC Acceptance . . . . . . . . . . . . . . 107

5.2 .2 NC Topology Cuts . . . . . . . . . . . . . . . . 108

5.3 CC Selection . . . . . . . . . . . . . . . . 112

5.3 .1 CC Acceptance . . . . . . . . . . . . . . . . . . 112

5.3.2 kNN-Based CC/NC Separation ........... . 113

5.4 Selector Performance . . . . . . . . . . . . . . . . 120 
6 Systematic Uncertainties

6.1 Introduction . . . . . . . . . . . . . . . . . . 123

6.2 Detector-Related Systematic Uncertainties for CC and NC samples 124

6.2 .1 Relative Normalisation . . . . . . . . . . . . . . . . . . 124

6.2.2 Acceptance and Selection Efficiency in the ND . . . . . . . 125

6.2.3 Track and Shower Energy Scale . . . . . . . . . . . 127

6.2.4 Poorly-Reconstructed Events . . . . . . . . . . . . . 129

6.2.5 Neutrino Cross-Sections . . . . . . . . . . . . . . . 130

6.2.6 CC and NC Backgrounds . . . . . . . . . . . . . . 132

6.2.7 The FD Cleaning and Cosmic Uncertainties . . . . . . . . 133

6.3 Creation of the Covariance Matrices . . . . . . . . . . . . . . . 134

6.4 Hadron Production . . . . . . . . . . . . . . . . . . . . 135

6.5 Beam Focusing . . . . . . . . . . . . . . . . . . . . . . . 138

6.6 Total CC and NC Systematic Uncertainties . . . . . . . . . . . . 142

7 The MINOS Sterile Neutrino Analysis 146

7.1 Sterile Neutrino Oscillations at MINOS . . . . . . . . . . . . . . 146

7.2 The MINOS Neutrino Energy Spectra . . . . . . . . . . . . . . . . 151

7.2.1 The MINOS Dataset Epoch . . . . . . . . . . . . 151

7.2 .2 Inter-Detector Flux Differences . . . . . . . . . . . . . 152

7.2.3 Far Detector Extrapolation . . . . . . . . . . . . . 153

7.2.4 Neutral Current $R$-Values at the Far Detector . . . . . . . 155

7.3 Fitting with the Far-over-Near Ratio . . . . . . . . . . . . . 158

7.3.1 Near Detector Oscillations . . . . . . . . . . . . . 158

7.3.2 The $\chi^{2}$ Expression . . . . . . . . . . . . . . 161

7.3.3 Performing the Fit . . . . . . . . . . . . . . . 164

7.3.4 The Likelihood Surfaces . . . . . . . . . . . . . . 165

7.3.5 Degeneracies in the $3+1$ Sterile Neutrino Model . . . . . . 166

7.3.6 Confidence Limits with Exact Coverage . . . . . . . . . . . 169 
7.3.7 Sterile Mixing Angle Limits with a Fixed $\Delta m_{41}^{2} \ldots \ldots$. . 171

7.4 Effect of Systematic Uncertainties . . . . . . . . . . . . . 173

7.5 Combination with the Bugey-3 Reactor Experiment . . . . . . . . 175

8 Feldman-Cousins Confidence Intervals $\quad 179$

8.1 Introduction . . . . . . . . . . . . . . . . . . . . 179

8.2 Some Statistical Theory _. . . . . . . . . . . . . . . . 180

8.2.1 It began with Neyman the Frequentist . . . . . . . . . 180

8.2.2 Why not take a Bayesian Approach? . . . . . . . . . . 181

8.3 Global and Raster Scans . . . . . . . . . . . . . . . . . . . . 182

8.4 Does the MINOS Sterile Neutrino Analysis need an FC correction? 186

8.5 The Feldman-Cousins Procedure . . . . . . . . . . . . . . . . . 189

8.5.1 Applying the Feldman-Cousins procedure to the Sterile Neutrino Analysis . . . . . . . . . . . . . . 190

8.6 Statistical and Systematic Fluctuations in Fake Data . . . . . . 196

9 Near Detector Data Quality Monitoring 199

9.1 Data Quality Monitoring at the ND . . . . . . . . . . . . 199

9.1.1 Data Quality for the MINOS Era . . . . . . . . . 200

9.1.2 Data Quality for the MINOS+ Era . . . . . . . . . . 202

9.2 Does MINOS need an Intensity Correction? . . . . . . . . . . . 207

9.3 Calculating the Intensity Corrections . . . . . . . . . . . . . . 210

9.4 Proof of Linearity . . . . . . . . . . . . . . . . . . . 218

10 Conclusions and Future Outlook 224

10.1 Summary . . . . . . . . . . . . . . . . . . 224

10.2 Future Outlook . . . . . . . . . . . . . . . . 225

10.3 Conclusion . . . . . . . . . . . . . . . . . . 226

$\begin{array}{lr}\text { Appendices } & 228\end{array}$ 
A.1 PMNS Matrix for a 3+1 Model . . . . . . . . . . . . . . . . 229

A.2 Neutrino Oscillation Probabilities . . . . . . . . . . . . . 231

A.2.1 Muon neutrino survival: $P\left(\nu_{\mu} \rightarrow \nu_{\mu}\right) \ldots \ldots . \ldots 231$

A.2.2 Sterile neutrino appearance: $P\left(\nu_{\mu} \rightarrow \nu_{s}\right) \ldots \ldots 233$

A.2.3 Electron neutrino appearance: $P\left(\nu_{\mu} \rightarrow \nu_{e}\right) \ldots \ldots . . .235$

References

Word count: 37,240 


\section{List of Figures}

2.1 Measurements of the hadron production cross-section around the $Z$ resonance. . . . . . . . . . . . . . . . . . . 30

2.2 Prediction of the solar neutrino energy spectrum from the Standard Solar Model. . . . . . . . . . . . . . . . . . . . . . 34

2.3 Flux of $\nu_{e}$ compared to the flux of $\nu_{\mu}+\nu_{\tau}$ measured by the SNO experiment. . . . . . . . . . . . . . . 37

2.4 Short-baseline reactor-antineutrino anomaly. . . . . . . . . . . 50

2.5 LSND and MiniBooNE 90\% and 99\% allowed regions for sterile anti-neutrinos. . . . . . . . . . . . . . . 53

3.1 The MINOS neutrino beam-line. . . . . . . . . . . . . . 56

3.2 The number of protons on target and the beam configurations as a function of time for the MINOS and MINOS+ experiments. . . 57

3.3 Measurements of $\Delta m_{32}^{2}$ and $\theta_{32}$ from the MINOS three-flavour analysis. . . . . . . . . . . . . . . . . . . . . . 58

3.4 The neutrino energy spectra for $\nu_{\mu}$ charged-current events observed at the Near Detector for MINOS, MINOS+ and the NO $\nu$ A experiments. . . . . . . . . . . . . . . . . . . 59

3.5 The NuMI beamline. . . . . . . . . . . . . . . . . . . . . 60

3.6 The flux of neutrino parents as a function of true neutrino-energy at the Near Detector. . . . . . . . . . . . . . . 62 
3.7 The true energy distribution of Near Detector events from the three different NuMI configurations. . . . . . . . . . . . . . . 63

3.8 Energy spectra at the MINOS Near Detector for the $\nu_{\mu}$-dominated and $\bar{\nu}_{\mu}$-enhanced beams. . . . . . . . . . . . . . . . . . 64

3.9 An example of slip-stacking seen by the MINOS Near Detector in $6+3$ mode. . . . . . . . . . . . . . . . . . 65

3.10 Technical drawing of the NuMI target for MINOS running. . . . . 66

3.11 The arrangement of a plastic scintillator and steel plane in the MINOS detectors. . . . . . . . . . . . . . . 67

3.12 The MINOS Near Detector. . . . . . . . . . . . . . 68

3.13 The MINOS Far Detector. . . . . . . . . . . . . . . 70

3.14 A strip of MINOS scintillator with a wavelength-shifting fibre installed. . . . . . . . . . . . . . . . . 71

3.15 MINOS Near Detector scintillator modules. . . . . . . . . . . . . 72

3.16 MINOS Far Detector scintillator modules. . . . . . . . . . . . 73

3.17 Neutrino interaction topologies observed in the MINOS detectors. 77

3.18 Total muon-flavoured neutrino and anti-neutrino charged-current cross-sections. . . . . . . . . . . . . . . . 79

4.1 The attenuation of the wavelength-shifting fibre measured by both cosmic-ray muons and a $\gamma$ source. . . . . . . . . . . . . . . 85

4.2 The stopping power of muons in copper as a function of muon momentum. ................... 86

4.3 True energy lost per scintillator plane for a simulated cosmic muon. 87

4.4 The response of both Near and Far Detectors before, during and after calibration. . . . . . . . . . . . . . . . 88

4.5 Calorimetric response of the MINOS detectors to pions and electrons of different momenta. . . . . . . . . . . . . . . . 89 
4.6 The Near Detector reconstructed neutrino energy spectra for data and simulation with and without beam reweighing. . . . . . . 92

4.7 A simulated charged current event in the MINOS Far Detector. . 95

4.8 A simulated neutral current event in the MINOS Far Detector. . . 96

4.9 Distributions of the ratio of reconstructed to true shower energies for various true shower energy ranges, comparing $k \mathrm{NN}$ to calorimetric shower energy estimation. . . . . . . . . . . . 100

4.10 Total systematic uncertainty on the $k \mathrm{NN}$ shower energy estimator as function of true shower energy. . . . . . . . . . . . . . . 101

5.1 Number of Far Detector events as a function of difference in time between the nearest spill to an event and the time of the first hit in the event. . . . . . . . . . . . . . . . 103

5.2 Maximum number of consecutive planes selection cut at the Near Detector. . . . . . . . . . . . . . . . 104

5.3 The pulse-height fraction pre-selection cut at the Near Detector. . 105

5.4 The fiducial volume at the Near Detector for neutral-current events.108

5.5 The fiducial volume at the Far Detector for neutral-current events. 109

5.6 Event-length cut at the Near Detector for neutral-current events. 110

5.7 Event-length cut at the Far Detector for neutral-current events. . 110

5.8 Neutral-current track-extension cut at the Near Detector. . . . . . 111

5.9 Neutral-current track-extension cut at the Far Detector. . . . . . 111

5.10 The fiducial volume at the Near Detector for charged-current events.113

5.11 The fiducial volume at the Far Detector for charged-current events. 113

5.12 Distribution at the Near Detector for the four input variables used to calculate the roID. . . . . . . . . . . . . . . 116

5.13 The roID variable distribution at the Near Detector. . . . . . . 117

5.14 Distributions at the Near Detector for the three additional input variables used to calculate the jmID. . . . . . . . . . . . 119 
5.15 The jmID variable distribution at the Near Detector.

5.16 Purity and efficiency for the charged-current selector for both the Far and Near Detectors. . . . . . . . . . . . . . . . . . . 12

5.17 Purity and efficiency for the neutral-current selector for both the Far and Near Detectors. . . . . . . . . . . . . . . . . . 121

6.1 Errors on the Far-over-Near ratios arising from uncertainties on the relative normalisation. . . . . . . . . . . . . . . . 125

6.2 Errors on the Far-over-Near ratios arising from acceptance uncertainties. . . . . . . . . . . . . . . 127

6.3 Errors on the Far-over-Near ratios arising from energy uncertainties.128

6.4 Error on the neutral current Far-over-Near ratio arising from uncertainties on the number of poorly-reconstructed events in the sample. . . . . . . . . . . . . . . . . 130

6.5 Errors on the Far-over-Near ratios arising from cross section uncertainties. . . . . . . . . . . . . . . . 132

6.6 Errors on the Far-over-Near ratios arising from the uncertainties on the levels of backgrounds. . . . . . . . . . . . . . . . 133

6.7 Error on the neutral current Far-over-Near ratio arising from uncertainties in the removal of noise, light injection, and cosmic muons. 134

6.8 Invariant differential cross section for $\pi^{+}$production at $x_{f}=0.025$ for NA49 data compared to Fluka simulation. . . . . . . . . . . . 136

6.9 Error bands on the charged-current neutrino energy spectra arising from uncertainties in hadron production. . . . . . . . . . . . . 137

6.10 Error bands on the neutral-current neutrino energy spectra arising from uncertainties in hadron production. . . . . . . . . . . . . . 139

6.11 Fractional change in Far-over-Near ratio arising from beam focusing uncertainties. . . . . . . . . . . . . . . . . . . . . . 142 
6.12 Error on the Far-over-Near ratios arising arising from beam focusing uncertainties. . . . . . . . . . . . . . . . . . . . 143

6.13 Total covariance matrix and error band for the charged-current sample. . . . . . . . . . . . . . . . . . . . 144

6.14 Total covariance matrix and error band for the neutral-current sample. . . . . . . . . . . . . . . . . . 145

7.1 The effects of three-flavour oscillation probabilities in the MINOS detectors . . . . . . . . . . . . . . . . 147

7.2 Neutrino oscillation probabilities for muon survival and sterile neutrino appearance with and without including matter effects. . . . 148

7.3 The effect of the $\mathrm{CP}$ violating phases in the $3+1$ model for neutralcurrent disappearance. . . . . . . . . . . . . . . . . . . . 150

7.4 The geometry of the decays of neutrino parents in the NuMI decay pipe. ....................... 153

7.5 The charged- and neutral-current reconstructed neutrino energy spectra in the Near Detector. . . . . . . . . . . . . . . . . . 154

7.6 The charged- and neutral-current reconstructed neutrino energy spectra in the Far Detector. . . . . . . . . . . . . . . . . 156

7.7 The $\nu_{\mu}$ charged- and neutral-current disappearance probabilities as a function of $L / E$ for various values of $\Delta m_{41}^{2} \ldots \ldots \ldots$. . . . 159

7.8 The measured charged- and neutral-current Far-over-Near ratios. . 160

7.9 Distance travelled by neutrinos that interact in the Near Detector. 161

7.10 The likelihood surface in sterile neutrino parameter space from a combined fit to the charged- and neutral-current data. . . . . . . 166

7.11 The likelihood surface in sterile neutrino parameter space from separate fits to the charged- and neutral-current data. 
7.12 The $90 \%$ and $95 \%$ C.L. exclusion contours from the MINOS data and sensitivity in the $\left\{\Delta m_{41}^{2}, \sin ^{2} \theta_{24}\right\}$ plane prior to a FeldmanCousins correction. . . . . . . . . . . . . . . 169

7.13 The MINOS 90\% and 95\% C.L. exclusion contours compared to other $\nu_{\mu}$ disappearance measurements. . . . . . . . . . . 170

7.14 The $\chi^{2}$ surface for the sterile neutrino mixing angle $\theta_{24}$ at a fixed value of $\Delta m_{41}^{2}=0.5 \mathrm{eV}^{2} \ldots \ldots \ldots \ldots \ldots \ldots \ldots$

7.15 The $\chi^{2}$ surface for the sterile neutrino mixing angle $\theta_{34}$ at a fixed value of $\Delta m_{41}^{2}=0.5 \mathrm{eV}^{2} \ldots \ldots \ldots \ldots \ldots \ldots \ldots$. . . . . . . . 172

7.16 The effects of the Individual systematic uncertainties on the $90 \%$ C.L. sensitivity. . . . . . . . . . . . . . . . . 174

7.17 The effects of the Incremental systematic uncertainties on the $90 \%$ C.L. sensitivity. . . . . . . . . . . . . . . . . 174

7.18 Bugey-3 90\% C.L. exclusion contour. . . . . . . . . . . . . 176

7.19 MINOS and Bugey-3 combined 90\% C.L. exclusion contour, compared to the results from the LSND and MiniBooNE experiments. 177

8.1 An example of the $90 \%$ C.L. construction with statistically fluctuated Far Detector fake data. . . . . . . . . . . . . . . . . . 187

8.2 The $\Delta \chi^{2}$ distribution for 133 fake experiments. . . . . . . . 188

8.3 Feldman-Cousins $\Delta \chi^{2}$ distribution along with best fit values. Fake data produced using the underlying true values of $\Delta m_{41}^{2}=4 \mathrm{eV}^{2}$ and $\theta_{24}=0.32 \ldots \ldots \ldots \ldots \ldots \ldots$

8.4 Feldman-Cousins $\Delta \chi^{2}$ distribution along with best fit values. Fake data produced using the underlying true values of $\Delta m_{41}^{2}=0.03 \mathrm{eV}^{2}$

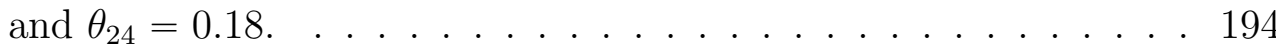

8.5 Feldman-Cousins correction surface used to construct a 90\% C.L. exclusion contour. . . . . . . . . . . . . . . . 195 
8.6 Comparison of the $90 \%$ C.L. exclusion contour from MINOS data and simulation before and after the application of the FeldmanCousins unified approach. . . . . . . . . . . . . . . . 196

8.7 Example of fluctuated fake Far-over-Near ratios. . . . . . . . . . . 197

9.1 Stability of the charged-current neutrino energy spectrum for all MINOS low-energy neutrino running. . . . . . . . . . . . . 200

9.2 Stability of the neutral-current neutrino energy spectrum for all MINOS low-energy neutrino running. . . . . . . . . . . . . . 201

9.3 Stability of the charged-current event rate for all MINOS lowenergy neutrino running. . . . . . . . . . . . . . . . 202

9.4 Stability of the neutral-current event rate for all MINOS lowenergy neutrino running. . . . . . . . . . . . . . . 203

9.5 Stability of the charged-current neutrino energy spectrum for MINOS+ Run 11. . . . . . . . . . . . . . . . . 204

9.6 Stability of the charged-current event rate for MINOS+ Run 11. . 204

9.7 Stability of the charged-current neutrino energy spectrum for MINOS+ Run 12. . . . . . . . . . . . . . . . 205

9.8 Stability of the charged-current event rate for MINOS+ Run 12. . 206

9.9 MINOS Run Period 1 batch structure. . . . . . . . . . . . . 208

9.10 MINOS Run Period 2 batch structure. . . . . . . . . . . . 208

9.11 MINOS Run Period 3 batch structure. . . . . . . . . . . . 208

9.12 MINOS Run Period 5 batch structure. . . . . . . . . . . . 209

9.13 MINOS Run Period 6 batch structure. . . . . . . . . . . . . 209

9.14 MINOS Run Period 10 batch structure. . . . . . . . . . . . . . . 209

9.15 A summary of the composition of the MINOS data for all lowenergy neutrino running showing the division of each Run period into high- and low-intensity batches. . . . . . . . . . . . 210 
9.16 Simulated energy spectra of charged- and neutral-current events (Run 3) for four different intensities. . . . . . . . . . . . . . 211

9.17 Ratios of simulated Near Detector energy spectra between high and low intensities, for both charged- and neutral-current events. . 212

9.18 Proportions of intensities used in the Near Detector Monte Carlo for all low-energy neutrino running. . . . . . . . . . . . . . . . . 214

9.19 The proton-per-batch intensities for all low-energy neutrino running. 215

9.20 The total intensity corrections to be applied to the Near Detector Monte Carlo for all low-energy neutrino running. . . . . . . . . . . 219

9.21 Linear fits to the Monte Carlo event rate as a function of intensity for Run Period 3. . . . . . . . . . . . . . . . . . . . . . 221

9.22 Comparison of the linearity of the charged-current event rate between data and Monte Carlo as a function of intensity. . . . . . . 222

9.23 Comparison of the linearity of the neutral-current event rate between data and Monte Carlo as a function of intensity. . . . . . . 223

10.1 Sensitivity of the sterile neutrino search with the addition of MINOS+ data. . . . . . . . . . . . . . . . 226

10.2 MINOS and MINOS+ sterile anti-neutrino sensitivity. . . . . . . . 227 


\section{List of Tables}

2.1 Values of oscillation parameters from a three-flavour global fit to experimental data. . . . . . . . . . . . . . . . . 4 46

5.1 Efficiency and purity of event selectors. . . . . . . . . . . . 122

7.1 Summary of the data collected in MINOS in terms of the protonson-target exposure. . . . . . . . . . . . . . . . . 152

7.2 Integrated number of neutrino events observed at the Far Detector. 157

7.3 $R$-values calculated from the Far Detector neutral-current neutrino energy spectrum. . . . . . . . . . . . . . . . 157

7.4 Values and constraints on neutrino oscillation parameters in the $3+1$ fit. . . . . . . . . . . . . . . 165

7.5 Summary of the best-fit parameter values at the combined chargedand neutral-current global minimum. . . . . . . . . . . . . . 168

8.1 $\chi^{2}$ quantile values for various confidence intervals for different numbers of degrees of freedom, $\alpha . \ldots \ldots \ldots$

9.1 The mean protons-per-batch and protons-on-target fraction in highand low-intensity batches for the MINOS dataset. . . . . . . . 216

9.2 The difference in intensity between data and simulation used for an intensity correction. . . . . . . . . . . . . . . 217 


\title{
Search for Sterile Neutrinos with the MINOS Long-Baseline Experiment
}

\author{
Ashley Michael Timmons \\ School of Physics and Astronomy \\ A thesis submitted to the University of Manchester for the degree of \\ Doctor of Philosophy \\ in the Faculty of Science and Engineering
}

2016

\begin{abstract}
This thesis will present a search for sterile neutrinos using data taken with the MINOS experiment between 2005 and 2012. MINOS is a two-detector on-axis experiment based at Fermilab. The NuMI neutrino beam encounters the MINOS Near Detector $1 \mathrm{~km}$ downstream of the neutrino-production target before travelling a further $734 \mathrm{~km}$ through the Earth's crust, to reach the Far Detector located at the Soudan Underground Laboratory in Northern Minnesota. By searching for oscillations driven by a large mass splitting, MINOS is sensitive to the existence of sterile neutrinos through looking for any energy-dependent perturbations using a charged-current sample, as well as looking at any relative deficit in neutral current events between the Far and Near Detectors. This thesis will discuss the novel analysis that enabled a search for sterile neutrinos covering five orders of magnitude in the mass splitting and setting a limit in previously unexplored regions of the parameter space $\left\{\Delta m_{41}^{2}, \sin ^{2} \theta_{24}\right\}$, where a $3+1$-flavour phenomenological model was used to extract parameter limits. The results presented in this thesis are sensitive to the sterile neutrino parameter space suggested by the LSND and MiniBooNE experiments.
\end{abstract}




\section{Declaration}

No portion of the work referred to in the thesis has been submitted in support of an application for another degree or qualification of this or any other university or other institute of learning. 


\section{Copyright}

The author of this thesis (including any appendices and/or schedules to this thesis) owns certain copyright or related rights in it (the "Copyright") and he has given The University of Manchester certain rights to use such Copyright, including for administrative purposes.

Copies of this thesis, either in full or in extracts and whether in hard or electronic copy, may be made only in accordance with the Copyright, Designs and Patents Act 1988 (as amended) and regulations issued under it or, where appropriate, in accordance with licensing agreements which the University has from time to time. This page must form part of any such copies made.

The ownership of certain Copyright, patents, designs, trade marks and other intellectual property (the "Intellectual Property") and any reproductions of copyright works in the thesis, for example graphs and tables ("Reproductions"), which may be described in this thesis, may not be owned by the author and may be owned by third parties. Such Intellectual Property and Reproductions cannot and must not be made available for use without the prior written permission of the owner(s) of the relevant Intellectual Property and/or Reproductions.

Further information on the conditions under which disclosure, publication and commercialisation of this thesis, the Copyright and any Intellectual Property and/or Reproductions described in it may take place is available in the University IP Policy ${ }^{1}$, in any relevant Thesis restriction declarations deposited in the University Library, The University Library's regulations ${ }^{2}$ and in The University's policy on Presentation of Theses.

\footnotetext{
${ }^{1}$ see http://documents.manchester .ac $\cdot$ uk/display $\cdot$ aspx?DocID=24420

${ }^{2}$ see http://www.library.manchester.ac.uk/about/regulations/
} 


\section{Acknowledgements}

The last four years have been an eventful time for me. I have been lucky enough to travel across the world meeting and working alongside many people who have all contributed in some way to the work in this thesis. It is impossible to name everyone but I believe some people deserve specific mention that without their support I would of struggled to complete my Ph.D.

First and foremost thanks goes to my supervisor Justin Evans. It is important to have a good relationship with ones supervisor and I could not of asked for better supervision. His patience, experience, and support shaped me into the scientist I am today.

I would like to thank the MINOS sterile neutrino analysis convenors João Coelho and Mike Kordosky for their helpful advice and ideas along the way. I would like to thank Andy Blake and Alex Sousa who were both analysis coordinators during my time at Fermilab. They both spent a significant amount of their time (out of their busy lives) to share with me their thoughts and advice which benefited me hugely, making me appreciate the impact you can have on someones understanding by spending the time to talk to them. Almost all ideas in physics stem from casual conversations around the workspace (usually with coffee nearby) and so I would like to thank Andy Perch, Alex Radovic, Adam Aurisano, Junting Huang, Adam Schreckenberger for all the great laughs and experiences we shared. Thanks to Robert Hatcher and Art Kreymer, their experience in computing massively benefited me. The MINOS collaboration has been a great pleasure to be apart of. The atmosphere and ethos towards encouraging young scientists to strive to their full potential contributed to the achievements I feel I have accomplished in my career so far. I would like to thank Karol Lang, Robert Plunkett and Jenny Thomas who were all the MINOS spokespeople at some point during my Ph.D.

I would like to thank everyone in the Manchester physics HEP department. During my two years in Chicago even though I was across the atlantic I still felt apart of my home intuition and in particular I would like to thank Stefan 
Söldner-Rembold for being easily approachable and handing any problems I encountered. Thanks goes to Sabah Salih for his entertaining conversations and technical support throughout my time at Manchester.

Family and friends played an important role in my Ph.D. At times the workload was tough and thanks to the love and support to those closest to me I was able to continue onwards and upwards. A special thanks to my long time friend Satnam Virdee for always being there. I also thank everyone in the "fake christmas" group, consisting of a wonderful collation of friends who always cheered me on and believed in me. I would like to thank my Dad, Sharon, and Jonathan for their love and support and for putting up with my persistent FaceTime calls whilst I was based in Chicago.

Unfortunately my mother passed away not long before the start of my $\mathrm{Ph}$.D. At first things looked bleak and I could not see how I would have the strength to get through my Ph.D. However, the love and support of my sister, Kelly, her husband, Adam, along with my niece, Harriet, and nephew, Jack, was enough to help me overcome all difficulties I have encountered - thank you very much. 


\section{Dedication}

I would like to dedicate this thesis to my mother, Maureen Timmons. I hope this achievement accurately reflects the sacrifices you made to ensure I received the opportunities in my life to do whatever I wanted. 


\section{Chapter 1}

\section{Introduction}

Neutrinos are the second most abundant particles in the universe, yet despite their ubiquitous presence they are proving to be one of the most difficult fundamental particles to study. The existence of the neutrino was not postulated until the 1930s and due to the neutrino interaction cross-sections being around ten orders of magnitude smaller than those of the lepton family the detection of the neutrino was not achieved until the late 1950s.

The challenge plaguing particle physicists interested in neutrinos is due to their almost almost negligible mass (neutrinos were considered massless until the late 1990s), and their infrequent weak interactions. Yet despite all these difficulties neutrino physics is emerging as a popular topic with many current and future experiments being dedicated to the properties of the neutrino.

It has been through the study of neutrino physics that the only beyondthe-Standard-Model physics has been observed: the fact that neutrinos oscillate between flavours as a function of distance and neutrino energy. This discovery implied that neutrinos must be massive, leading to so-far-unsuccessful efforts to measure the neutrino mass.

This thesis contributes to the understanding of neutrino oscillation, a field with many unknowns still present. There have been several anomalous results 
in the neutrino community which, amongst other possible explanations, could be accounted for by the existence of sterile neutrinos. The most famous anomalous results come from the LSND and MiniBooNE experiments which are discussed in detail later on. This thesis focuses on the topic of sterile neutrinos and presents an analysis that investigates the sterile neutrino oscillation parameter space suggested by these experiments.

Chapter 2 will introduce the history of the neutrino and the mathematical framework necessary to explain neutrino oscillation. This chapter will introduce the addition of sterile neutrinos to the current three-flavour model.

MINOS (the Main Injector Neutrino Oscillation Search) is described in detail in chapter 3 along with a description of the NuMI (Neutrinos at the Main Injector) facility. The NuMI beam delivers a neutrino beam that is observed by the MINOS experiment using two detectors separated by a distance of $734 \mathrm{~km}$. It is through the measurement of the reconstructed neutrino energy spectra at both detectors and any differences between those energy spectra that information about neutrino oscillations can be inferred.

Muon neutrinos interacting in the MINOS detectors can produce muon tracks and hadronic showers. A reconstructed neutrino energy spectrum can be measured by directly measuring the energies of any final state muon track and hadronic showers. To correctly measure these properties a calibration chain is implemented, which is described in chapter 4.

Neutrinos interact within the MINOS detectors through either the chargedcurrent or neutral-current interactions. From the event topology it is possible to discriminate between these two event types. This thesis utilises both interactions with chapter 5 discussing the topological cuts implemented and sophisticated algorithms used such that isolated samples of neutral-current and $\nu_{\mu}$ chargedcurrent events can be obtained.

All experimental analyses are plagued with some systematic uncertainties, 
that is, unknown quantities due to poorly-understood modelling that needs to be accounted for. A description of all systematic uncertainties relating to both the charged- and neutral-current samples is presented in chapter 6, with my contribution being work on the beam focusing systematic uncertainties.

Chapter 7 describes the fit, which I developed, that is performed on the two event samples using the ratio of the reconstructed neutrino energy spectra measured at both MINOS detectors. A fit is performed to probe a particular sterile neutrino model, the $3+1$ model. From this, limits are obtained that place constraints in the $\left\{\Delta m_{41}^{2}, \sin ^{2} \theta_{24}\right\}$ parameter space and also on the angle $\sin ^{2} \theta_{34}$. To enable a direct comparison to the sterile neutrino parameter space suggested by LSND and MiniBooNE a combination with a neutrino reactor experiment such as Daya Bay or Bugey-3 can be performed. Such a combination is presented at the end of chapter 7 .

The statistics of the MINOS $\nu_{\mu}$-disappearance analysis are studied in chapter 8 to understand any deviations from traditional confidence limit methods used that assume Gaussian statistics. The Feldman-Cousins unified approach is used to correctly set the confidence intervals. I address concerns of an intensity correction required for the data used in this thesis in chapter 9 along with the validation of data quality at the MINOS Near Detector for the data used in this thesis along with the first year of the MINOS+ era.

Finally, chapter 10 gives a summary of this thesis along with a conclusion on the results presented. A discussion on the future prospects for MINOS and the addition of MINOS+ data is given, focusing on how it will build upon the work presented in this thesis. 


\section{Chapter 2}

\section{Neutrino Physics}

This chapter begins by introducing the concept of the neutrino as a fundamental particle and the historical background since the neutrino was first postulated. This is followed by the mathematical interpretation of one of the surprises of the 21st century, neutrino oscillation, by which one can calculate the probability of a neutrino transitioning between flavour states. The last section will introduce the sterile neutrino into this mathematical model, a neutrino species even more difficult to detect (indirectly) than the elusive active neutrinos the scientific community is already aware of. A brief summary is given of the experimental data that first began to support theories of sterile neutrinos.

\subsection{The History of the Neutrino}

\subsubsection{The First Hints of a Neutrino}

The first mention of the neutrino was by another name, in 1930. Wolfgang Pauli postulated the existence of an undetectable (at the time) neutral particle called the "neutronen" (or neutron) that could solve the beta-decay kinematic problem [1]. The problem arose when in 1914 James Chadwick measured a continuous energy spectrum of electrons from beta decay using a magnetic spectrometer [2]. 
At the time this process was considered to be a two-body process; as such, one would expect a mono-energetic distribution. Pauli's suggestion of an additional undetectable particle,

$$
n \rightarrow p+e^{-}+\nu
$$

means this particle would take away some of the decay energy turning the electron energy distribution into the continuous spectrum of a three-body decay. Pauli did not differentiate between what we now know as the neutron inside a nucleus and the neutrino. He considered both to be the same particle.

It was not until 1932, when Chadwick discovered the neutron $[3,4]$ by observing the radiation emitted from beryllium upon bombarding the metal with alpha particles, that the neutrino and the neutron were understood as different particles. The radiation emitted was non-ionising and therefore must be neutral. Initial thoughts were that the radiation could be gamma rays. However, by placing paraffin wax as an absorber in the path of the radiation the wax was found to emit protons (paraffin wax consists mainly of hydrogen). By measuring the speeds and energies of these protons, Chadwick concluded that photons could not have provided the energies required to so easily eject the protons from the wax; and thus, the neutron was discovered.

Around the same time Francis Perrin [5] and Enrico Fermi [6] both put forward the idea that the neutrino is involved in beta decay, with the idea that the neutrino be massless. Much later, in 1968, Fermi did additional work in which he showed the dependence on the neutrino mass of the energy spectrum of the electron emitted in beta decay [7]. As a result the neutrino gained its own identity in particle physics. 


\subsubsection{The Neutrino is Discovered}

The discovery of the neutrino came about thanks to the efforts of Fred Reines and Clyde Cowan at the Hanford reactor site in 1953 [8, 9, 10, 11]. The discovery was made through inverse beta decay, $\bar{\nu}+p \rightarrow n+e^{+}$, by which a neutrino would interact with a proton resulting in a free neutron and a positron in the final state. The positron would undergo annihilation with an electron resulting in a photon pair; the neutron at a later time would then be absorbed by a nucleus resulting in a delayed photon signal. Reines and Cowan detected this by filling a tank with 300 litres of cadmium-doped liquid scintillator, surrounded by photomultiplier tubes (PMTs), situated next to a neutrino source (a nuclear rector). The PMTs detected the photon pair-production and the delayed photon signal once a neutron had been absorbed by the cadmium. It would be this double coincidence signal that would provide the signal for inverse beta decay.

Reines and Cowan counted the number of times a double coincidence was detected over a fixed time period $t$. This procedure was performed with the reactor switched on and again once switched off. The idea was to observe a deficit in the counting rate once the nuclear reactor (the neutrino source) was switched off. A net counting rate of $0.41 \pm 0.20$ counts $/$ min was the difference observed; this should be compared to the their predicted $\sim 0.2$ count $/$ min due to neutrinos, using a theoretical cross section of $6 \times 10^{-44} \mathrm{~cm}^{2}$. This result was not enough to irrefutably claim a discovery due to a larger than expected cosmic background. Their results motivated additional work.

Reines and Cowan performed a further experiment at the Savannah River Plant in 1956 [12]. They searched for the same inverse beta decay process, however they used three layers of plastic scintillator alternated with cadmium-doped water. This modified approach helped to provide a cosmic veto. A larger, more significant signal was observed and thus the neutrino was officially detected. More specifically, it was the electron antineutrino $\bar{\nu}_{e}$. 


\subsubsection{Different Neutrino Types}

In 1959 a different neutrino type, the muon neutrino, $\nu_{\mu}$, was predicted by Bruno Pontecorvo [13], motivated by the earlier discovery of the leptonic muon in 1939 by Carl Anderson and Seth Neddermeyer [14, 15].

The muon neutrino was discovered in 1962 in Brookhaven with the use of the world's first neutrino beam [16]. By colliding a high-energy proton beam onto a beryllium target secondary mesons were produced (predominantly pions). The (unfocused) pions subsequently decayed, producing a muon and muon neutrino in the final state, $\pi^{ \pm} \rightarrow \mu^{ \pm}+\nu_{\mu}\left(\bar{\nu}_{\mu}\right)$. Due to the weakly-interacting nature of the neutrino, an absorber could be placed in front of the final state particles and the muons and any remaining mesons would be absorbed whilst the neutrinos passed through.

A 10-ton aluminium spark chamber was situated in the neutrino beam, which could differentiate muons from electrons. The spark chamber observed 113 events. Of those 113 events, 34 events were identified as events with a single muon track of which 5 were considered to be the cosmic-ray background. if $\nu_{\mu}=\nu_{e}$ then there should be of the order of 29 events with an electron shower. However, only 6 electron-shower events were observed. These results implied the neutrino type created from the neutrino beam was different from that seen by Reines and Cowan.

History repeated itself again with the discovery of the much heavier leptonic tau particle, $\tau$, in 1975 using the SPEAR $e^{+} e^{-}$collider [17]. This naturally led to the question of the existence of a third neutrino type, the tau neutrino, $\nu_{\tau}$.

Tau neutrinos proved to be extremely difficult to produce and detect and the first indication of their existence came from an indirect measurement of the total number of neutrinos by the Large Electron Positron (LEP) collider at CERN. A comparison of the total width of the $Z$ boson compared to the width from its decays to visible particles yielded the width for its decay to neutrinos. Under 
the assumption that the coupling strength is equal between all neutrino types (universality) the fit to the number of neutrino types (for neutrinos with a mass lighter than $M_{Z} / 2$ ) was found to be $2.9840 \pm 0.0082$ [18] and is shown in figure 2.1.

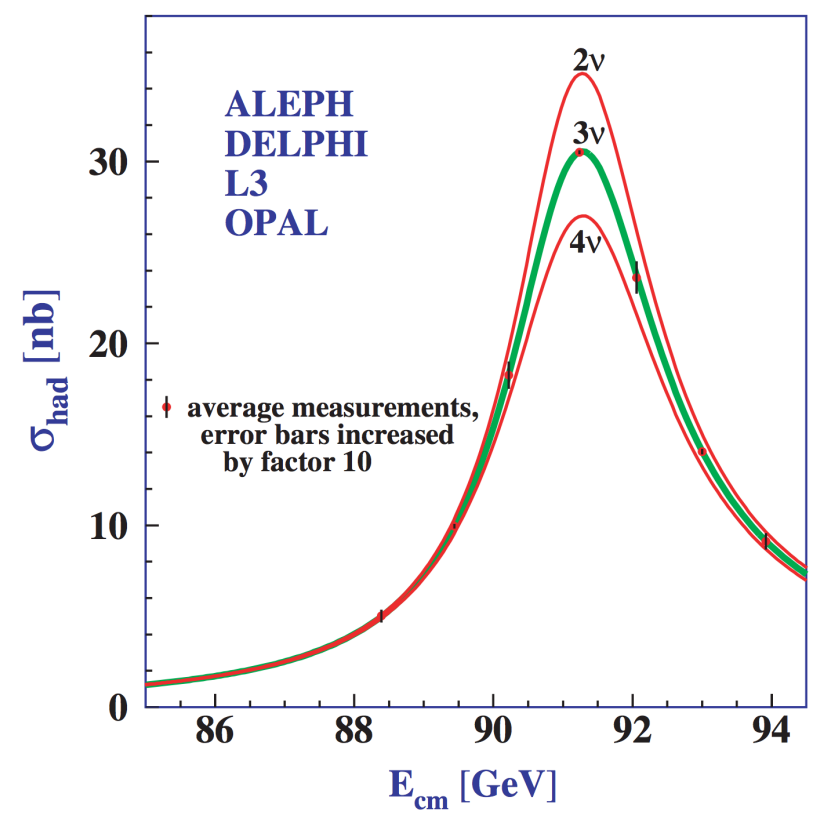

Figure 2.1: Measurements of the hadron production cross-section around the $Z$ resonance by the ALEPH, DELPHI, L3 and OPAL experiments at LEP. The coloured curves represent the predicted cross-section for models assuming two, three or four neutrino species within the Standard Model. Graph taken from [18].

The direct observation of the tau neutrino was finally achieved in 2001 by the DONUT (Direct Observation of the NU Tau) collaboration at Fermilab [19]. A beam containing tau neutrinos was created by colliding high-energy protons onto a thick tungsten target. The thick target allowed for the absorption of any pion or koan hadronic final state particles, resulting in a beam of strange-type $D$ mesons $\left(D_{s}\right)$. Appropriate shielding was installed to allow for the removal of any non-neutrino particles. The branching ratio for the decay $D_{s} \rightarrow \tau+\nu_{\tau}$ is $5.6 \%$. The detector itself was composed of alternating steel and emulsion planes, with which the tau neutrinos would interact, resulting in $\nu_{\tau}+N \rightarrow \tau^{-}+X$, where $N$ is the nucleus within the detector and $X$ is the final state hadronic shower. The emulsion allowed the tau lepton to be tracked. Due to the short lifetime of the 
tau a characteristic "kink" was searched for, from the tau having travelled a few millimetres before decaying. The emulsion allows for a high-resolution image of the decay process.

The DONUT experiment analysed 203 recorded neutrino interactions. They observed four tau neutrino interactions in the detector with an estimated background of 0.34 events.

\subsubsection{Properties of the Neutrino}

The neutrino interacts via the weak interaction through the exchange of the $W^{ \pm}$ and $Z$ bosons. Up until 1956 it was widely accepted that nature should not care whether a coordinate system is right-handed or left-handed. Without any experimental justification weak interactions were believed to conserve parity [20]. However, in 1957 parity violation was observed for the first time by Chen-Shiung $\mathrm{Wu}[21]$. Wu found that the angular distributions of electrons emitted via the decay of ${ }^{60} \mathrm{Co}$ are strongly correlated with the direction of the spin of the nuclei.

The implications of these results suggested all neutrinos are left-handed and all antineutrinos are right-handed. The definition of left- and right-handedness is determined by the helicity of the particle, defined as the direction the particle spins, $\boldsymbol{\sigma}$, in relation to its momentum, p. A right-handed particle would have both the spin and momentum aligned in the same direction, giving a positive helicity $(\boldsymbol{\sigma} \cdot \mathbf{p}>0)$, whereas a left-handed particle would have its spin and momentum anti-aligned $(\boldsymbol{\sigma} \cdot \mathbf{p}<0)$. One can argue that depending on the frame of reference taken, a particle could flip the direction of its momentum vector, leading to helicity not being Lorentz invariant. The quantity of chirality is therefore defined which is Lorentz invariant. However in the limit of zero mass (such as for the neutrino) both quantities are equal.

The first direct measurement of the helicity of the neutrino was in 1957 by Maurice Goldhaber, Lee Grozdins and Andrew Sunyar [22]. The measurement 
of the neutrino helicity was measured by looking at a sample of ${ }^{152} \mathrm{Eu}$, which undergoes K-electron capture. The process is shown in equation 2.2 along with the associated spins:

$$
\begin{aligned}
& { }^{152} \mathrm{Eu}+e^{-} \rightarrow{ }^{152} \mathrm{Sm}^{*}+\nu_{e} \rightarrow{ }^{152} \mathrm{Sm}+\gamma+\nu_{e} \\
& \begin{array}{lllllll}
0 & \frac{1}{2} & 1 & -\frac{1}{2} & 0 & 1 & -\frac{1}{2}
\end{array}
\end{aligned}
$$

The total spin of the system must sum to $\frac{1}{2}$, the initial spin state of the Kelectron. The ${ }^{152} \mathrm{Sm}^{*}$ experiences recoil in the opposite direction to that of the emitted neutrino. The ${ }^{152} \mathrm{Sm}^{*}$ radiates photons isotropically; a fraction of these photons will be opposite to the direction of the emitted neutrino. It is this fraction of slightly boosted photons (from the Sm recoil) that take away the total nuclear spin, giving the photons the same helicity as the neutrino. These selected photons pass through a block of magnetised iron which can more easily absorb photons with a spin aligned with the magnetic field. The photons that traverse the magnetised iron then strike the ${ }^{152} \mathrm{Sm}$ nuclei in a $\mathrm{SmO}_{2}$ target. The fluoresced state de-excites by emitting gamma rays which are subsequently detected. It is by measuring the direction and helicity of the photons that one can infer the helicity of the neutrino. The results from this test showed that the helicity of the photons, and therefore the neutrinos, were all consistent with being left-handed.

\subsection{Neutrino Oscillations}

The idea of neutrino oscillations was first proposed by Bruno Pontecorvo in $1957[23,24]$. By analogy with the oscillations observed in the Kaon system, Pontecorvo considered the idea of neutrino flavour change from one type to another allowed by a non-zero mass. The neutrino mass was still not measured and widely believed to be zero, however the mere observation of neutrino oscillations 
would imply that neutrinos are massive particles.

In 1962 Ziro Maki, Masami Nakagawa and Shoichi Sakata developed the model of neutrino mixing that we now use [25]. At this point in time there was no evidence to suggest neutrinos oscillate. However, this work would prove to be fundamental.

\subsubsection{Solar Neutrinos}

The first evidence to hint at neutrino flavour change came from the study of solar neutrinos in 1968 in the form of an experiment performed by Ray Davis at the Homestake mine [26]. By filling a tank with $\mathrm{C}_{2} \mathrm{Cl}_{4}$ the flux of solar neutrinos from ${ }^{8} \mathrm{~B}$ decay could be measured by searching for Argon atoms: $\nu_{e}+{ }^{37} \mathrm{Cl} \rightarrow{ }^{37} \mathrm{Ar}+e^{-}$ (threshold $814 \mathrm{keV}$ ). Figure 2.2 shows the predictions of solar neutrino fluxes as a function of neutrino energy from the Standard Solar Model (SSM) [27]. Davis set a limit on the event rate of $\leqslant 3 \times 10^{-36} \mathrm{~s}^{-1}$ per ${ }^{37} \mathrm{Cl}$ atom. Davis concluded from his result that he saw a deficit in the neutrino event rate around half that predicted by solar models of $7.5 \times 10^{-36} \mathrm{~s}^{-1}$ per ${ }^{37} \mathrm{Cl}$ atom [27].

Initial doubts came from the reliability of the solar model to accurately predict the neutrino flux from the ${ }^{8} \mathrm{~B}$ chain, mainly due to the large uncertainties on the poorly-known nuclear cross-sections at low energies [29]. In the SSM the prediction of the neutrino flux from the ${ }^{8} \mathrm{~B}$ chain has a total uncertainty of $30 \%$ [30]. The fraction of neutrino flux from the ${ }^{8} \mathrm{~B}$ chain compared to the total flux is $\sim 0.08 \%$ [31], a number so small it was questionable if this could be a channel from which one could produce reliable results. Davis continued his work with solar neutrinos which over the years yielded similar results [32]. It was not for another 20 years, in 1986, that another experiment, the Kamiokande water Cherenkov detector (originally built to search for proton decay) would make a measurement of the solar neutrino flux from the ${ }^{8} \mathrm{~B}$ chain via the method of electron scattering, $\nu_{e}+e^{-} \rightarrow \nu_{e}+e^{-}$. The first solar neutrino flux measurement 


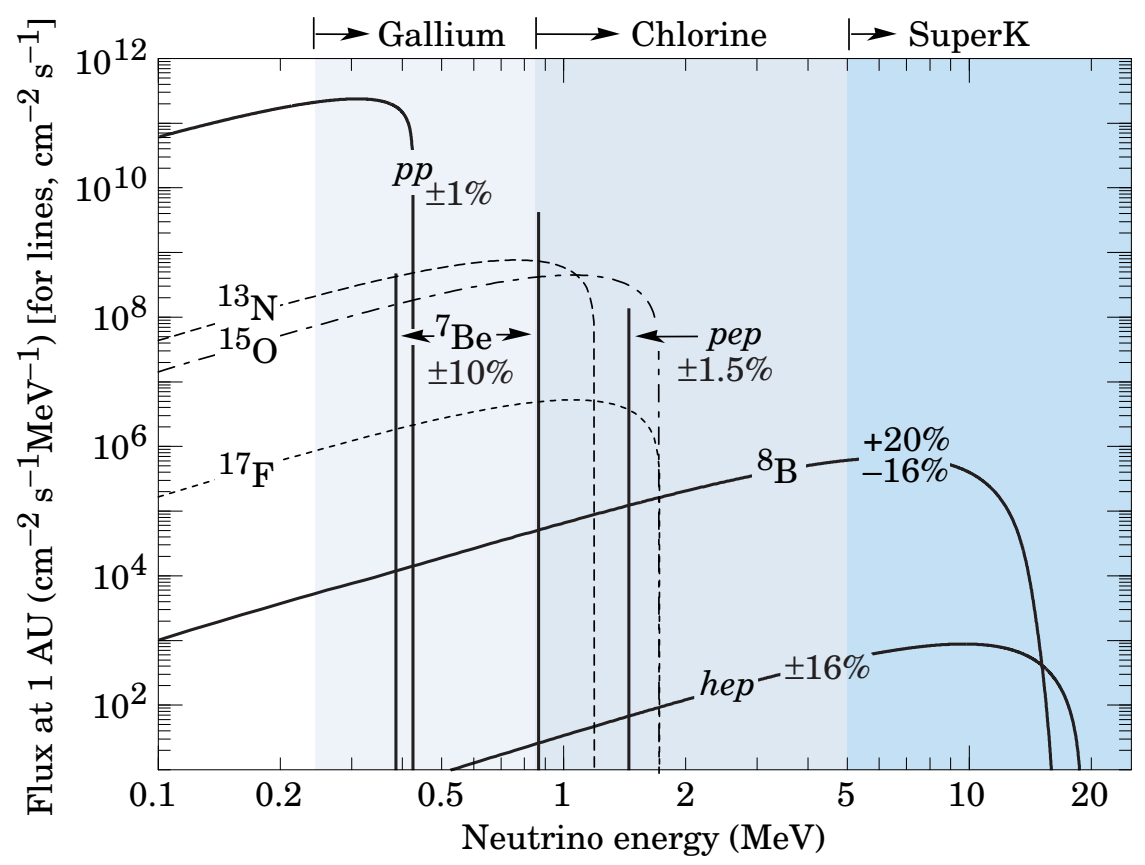

Figure 2.2: Prediction of the solar neutrino energy spectrum from the Standard Solar Model. The shaded regions indicate the energy ranges over which various experiments are sensitive. Graph taken from [28].

from Kamiokande agreed with the previous measurement by Davis and observed a neutrino flux of $0.46 \pm 0.13$ (stat.) \pm 0.08 (syst.) times the flux expected from the SSM [33].

Once again, the large uncertainties associated with the SSM for the predicted flux of neutrinos from the ${ }^{8} \mathrm{~B}$ chain were brought into question. Further investigation was warranted into what was becoming the "solar neutrino problem" with additional experiments attempting to measure the lower-energy solar neutrinos from the $p p$ chain. Although harder to detect experimentally the associated uncertainty in the prediction of the flux by the SSM was much lower at $\sim 3 \%$ [30]. In 1991 the Soviet-American Gallium Experiment (SAGE) [34], and in 1992 the Gallium Experiment (GALLEX) [35], measured the neutrino flux from the $p p$ chain using gallium as the interaction medium: $\nu_{e}+{ }^{71} \mathrm{Ga} \rightarrow e^{-}+{ }^{71} \mathrm{Ge}$ (threshold $233 \mathrm{keV}$ ). The use of Gallium allows for a detector to have a lower energy threshold for detecting solar neutrinos; the range can be seen in figure 2.2. The 
conclusion from their final results $[36,37]$ was in agreement with the previous discrepancies, observing a deficit of solar neutrinos of a factor of $\sim 2$. Further experimental results would add to the tension between the predicted flux from the SSM and that measured experimentally [38, 39, 40, 41].

During the period from 2000 up to 2006, the Sudbury Neutrino Observatory (SNO) experiment collected data that would shed light onto the previous measurements of the deficit observed for the measured solar neutrino flux. The SNO experiment consisted of a sphere of 1 kton of heavy water $\left(\mathrm{D}_{2} \mathrm{O}\right)$ surround by PMTs. By using heavy water as an interaction medium the SNO experiment could search for multiple neutrino interactions channels:

$$
\begin{array}{ll}
\nu_{e}+d \rightarrow p+p+e^{-} & \text {(charged current) }, \\
\nu_{e}+e^{-} \rightarrow e^{-}+\nu_{e} & \text { (charged current), } \\
\nu_{x}+d \rightarrow p+n+\nu_{x} & \text { (neutral current) }, \\
\nu_{x}+e^{-} \rightarrow e^{-}+\nu_{x} & \text { (neutral current) },
\end{array}
$$

where $\nu_{x}$ denotes a neutrino of possible flavour type $e, \mu, \tau$. The final state electrons from the charged current processes in equations 2.3 and 2.4 can be observed if the velocity of the electron is greater than the speed of light in the medium, in which case a Cherenkov light ring is produced. An NC interaction results in a free neutron in the final state which captures on a nucleus in the detector and results in the production of one or more gamma-rays. The gamma-rays will usually Compton-scatter an electron which can be detected due to the presence of a Cherenkov light ring. The time delay between the time of the NC interaction and the detection of the electron from Compton scattering is of the order of tens of milliseconds.

To increase the sensitivity to the NC interactions a "salt phase" was intro- 
duced where approximately $2000 \mathrm{~kg}$ of $\mathrm{NaCl}$ was mixed into the heavy water. This increased the neutron capture efficiency as the neutron capture cross section for ${ }^{35} \mathrm{Cl}$ is larger than that of heavy water.

By measuring both the CC and NC interactions SNO could measure the $\nu_{e}$ flux as well as the total neutrino flux $\left(\nu_{e}+\nu_{\mu}+\nu_{\tau}\right)$. The results from the SNO experiment are shown in figure 2.3. The figure shows the flux of $\nu_{\mu}+\nu_{\tau}$ compared to the $\nu_{e}$ flux. The dotted lines indicate a region such that the total neutrino flux sums to the SSM. The red band is the $\nu_{e}$ flux measured by detecting charged-current interactions, which is sensitive only to electron neutrinos. The green band is the electron neutrino scattering analysis which has some sensitivity to other neutrino flavours but is $\sim 6$ times more sensitive to electron neutrinos. The corresponding Super-Kamiokande measurement is superimposed in grey. The ratio of the $\nu_{e}$ flux comapred to that of the total neutrino flux measured by SNO was $0.340 \pm 0.023$ (stat.) ${ }_{-0.031}^{+0.029}$ (syst.) [42].

The results show a non-zero component for the flux of $\nu_{\mu}+\nu_{\tau}$ which is an indication of neutrino flavour change. This is supported by the decreased $\nu_{e}$ flux and the total neutrino flux being consistent with that predicted by the SSM. This was strong evidence for neutrino oscillations and was the beginning of a new field of study into the properties of neutrinos with the implication that neutrino have a non-zero mass.

\subsubsection{Atmospheric Neutrinos}

In parallel with the on-going solar neutrino problem, evidence for disappearance of neutrinos created within the Earth's atmosphere was emerging. Atmospheric neutrinos are produced by primary cosmic rays striking the atmosphere producing 


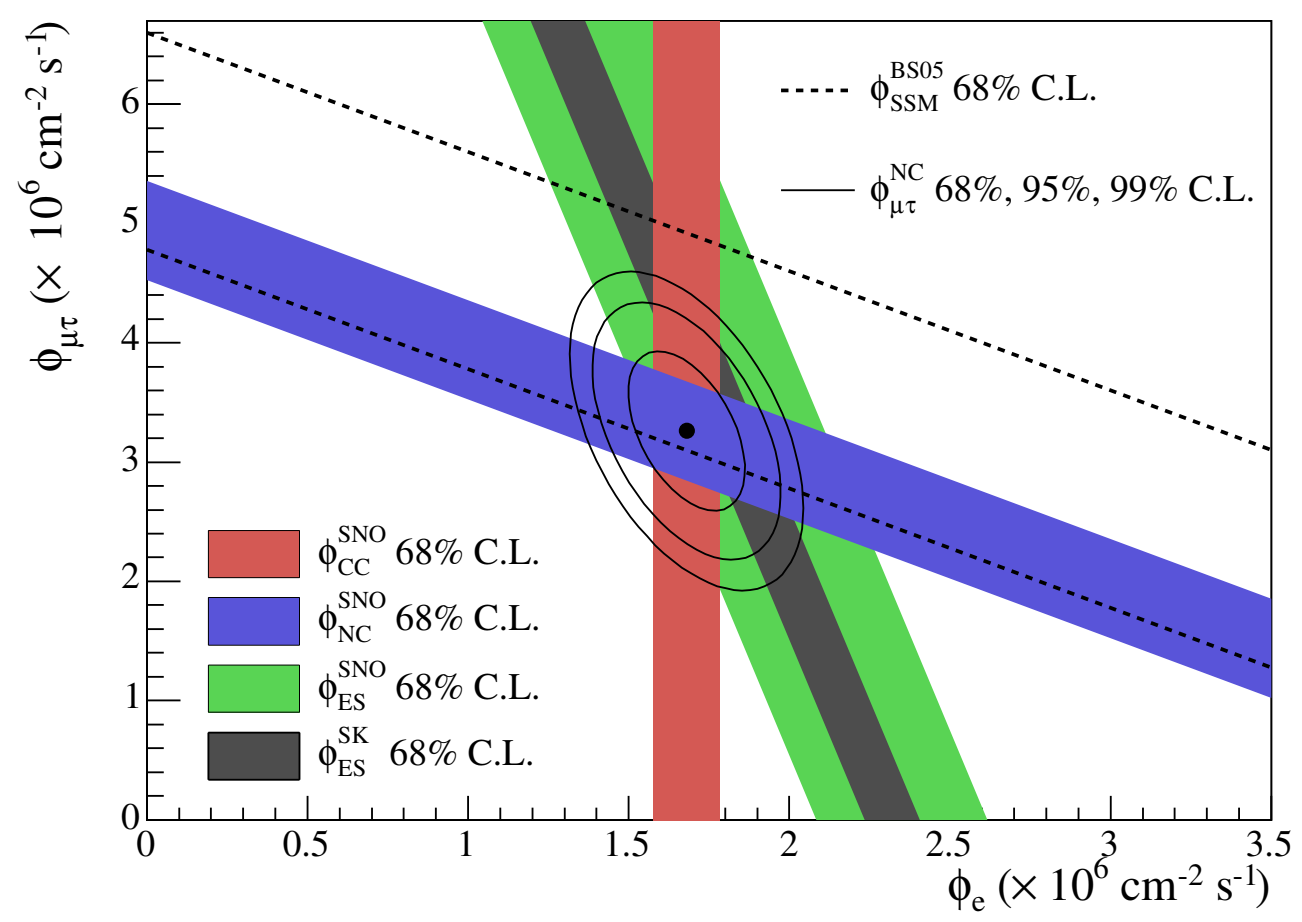

Figure 2.3: Solar neutrino fluxes measured by SNO. The dotted lines indicate a region such that the total neutrino flux sums to the SSM. The red band is the $\nu_{e}$ flux from detecting charged current interactions, which is sensitive only to electron neutrinos. The green band is the electron neutrino scattering analysis with the corresponding Super-Kamiokande measurement superimposed in grey. The point shows the best fit values for the $\nu_{e}$ flux and the $\nu_{\mu}+\nu_{\tau}$, with $68 \%$, 95\%, and 99\% C.L. contours included. Graph taken from [42].

pions and kaons which then decay. This is predominantly the decay of pions,

$$
\begin{aligned}
\pi^{+} \rightarrow \mu^{+}+\nu_{\mu} & \\
& \left\llcorner e^{+}+\nu_{e}+\bar{\nu}_{\mu},\right.
\end{aligned}
$$

with a similar channel for $\pi^{-}$decay and a contribution from kaons. The neutrinos produced span a wide range of energies from sub-GeV to beyond $\mathrm{TeV}$. The expected ratio of muon flavour to electron flavour at $\mathrm{GeV}$ energies is

$$
\phi\left(\nu_{\mu}+\bar{\nu}_{\mu}\right): \phi\left(\nu_{e}+\bar{\nu}_{e}\right) \approx 2: 1
$$


In 1988 the Kamiokande-II experiment, as well as investigating the solar neutrino anomaly, also reported that, although the rate of electron-flavour neutrinos was in agreement with predictions, a deficit of muon-flavour neutrinos was observed [43]. The Kamiokande experiment saw the deficit in muon-neutrinos for up-going events, that is, neutrinos that enter the detector from below and therefore must have travelled through the Earth. This issue was to become known as the "atmospheric neutrino anomaly". This deficit of atmospheric neutrinos was confirmed by the Irvine-Michigan-Brookhaven (IMB) experiment in 1992 [44]. Both of these experiments were water-Cherenkov detectors and it was suggested that these types of experiments may be suffering from some systematic effect specific to their design. However in 1997 the iron-calorimeter Soudan 2 experiment also found a deficit of atmospheric muon-flavour neutrinos [45]. These observations all agreed on seeing only $2 / 3$ of what was expected of the atmospheric muon-neutrino flux for no oscillations.

\subsubsection{Reactor Neutrinos}

Nuclear reactors provide an intense source of man-made electron anti-neutrinos. The range of energies for the emitted anti-neutrinos is $1-10 \mathrm{MeV}$. For a $1 \mathrm{GW}$ nuclear reactor one can approximate around $2 \times 10^{20} \nu \mathrm{s}^{-1}$ emitted isotropically.

Nuclear reactor neutrino experiments detect $\bar{\nu}_{e}$ interactions through the inverse beta decay (IBD) process. The detectors typically consist of a central detector filled with liquid scintillator and sometimes loaded with Gd. An IBD event is identified by the positron energy deposit and the neutron-capture energy-signal that are both correlated in time. The Gd is mixed with the scintillator in order to increase the neutron capture probability with the energy of the $\bar{\nu}_{e}$ being calculated from the prompt energy of the positron. The threshold for IBD is $1.8 \mathrm{MeV}$ with reactor neutrino experiments seeing a peak in the $\bar{\nu}_{e}$ energy spectrum around $4 \mathrm{MeV}$. 
The neutrino oscillation reactor experiment, the Kamioka Liquid Scintillator Antineutrino Detector (KamLAND), published results in its first year of data taking in 2002 [46]. The ratio of the number of observed reactor $\bar{\nu}_{e}$ events to that expected in the absence of neutrino oscillations was $0.611 \pm 0.085$ (stat.) \pm 0.041(syst.), the first significant evidence for $\bar{\nu}_{e}$ disappearance.

\subsubsection{Accelerator Neutrino Beams}

Accelerator neutrino beams are another source of high-intensity man-made muontype neutrinos. The general setup consists of colliding a high-energy proton beam onto a nuclear target to obtain a beam of secondary charged pions and kaons which are permitted to decay in a decay pipe. The boosted hadrons decay in flight yielding a neutrino beam dominated by muon-flavoured neutrinos. To increase the flux of the neutrino beam, focusing horns are used to decease the divergence of the pions and kaons before they decay.

Depending on the accelerator neutrino beam configuration one can achieve a beam of neutrinos or anti-neutrinos over a wide range of energies, $E_{\nu}$, typically 1-200 GeV. The detectors are situated at a particular distance, $L$, such that the region of $L / E_{\nu}$ probed is similar to that experienced by the atmospheric neutrinos observed by Kamiokande. Usually, there are two detectors with different baselines; this allows for a reduction in the systematic uncertainties by observing the relative changes in the reconstructed neutrino energy spectra between the two detectors.

The first long-baseline neutrino oscillation experiment to use an accelerator neutrino beam was KEK-to-Kamioka $(\mathrm{K} 2 \mathrm{~K})$. K2K searched for $\nu_{\mu}$ disappearance with a neutrino flight distance of $250 \mathrm{~km}$. The data-taking period began in 1999 and continued untill 2004; K2K observed direct evidence for $\nu_{\mu}$ disappearance observing 112 beam neutrino events compared to the expected number without neutrino oscillation of $158.1_{-8.6}^{+9.2}[47]$. 
The second long-baseline accelerator experiment was the Main Injector Neutrino Oscillation Search (MINOS). MINOS has a longer baseline of $735 \mathrm{~km}$ and took data beginning in 2005 up until 2012. In the first year of data taking MINOS was already seeing significant evidence of $\nu_{\mu}$ disappearance [48] and up until today continues to publish results including the observation of the appearance of $\nu_{e}[49]$.

The disappearance of muon-flavoured neutrinos seen by atmospheric neutrino oscillation experiments indicates that the muon-neutrinos are changing flavour. One would therefore expect an increase in either the electron or tau flavoured neutrinos. The majority of muon-neutrinos are in fact oscillating into tau-neutrinos, with very little electron-neutrino appearance being observed. Tau-neutrino appearance is extremely difficult to observe, although has been seen by the Oscillation Project with Emulsion-tRacking Apparatus (OPERA) [50] experiment.

The success of accelerator neutrino sources has led to many experiments such as Tokai to Kamioka (T2K) [51] and the latest neutrino experiment NuMI OffAxis $\nu_{e}$ Appearance $(\mathrm{NO} \nu \mathrm{A})$ which published it first results in $2016[52,53]$. A more detailed description of the various accelerator-based neutrino beams can be found in [54].

\subsubsection{Oscillation Formalism}

The observation of neutrino oscillation and its dependance on the neutrino path length and energy confirmed that neutrinos are massive. For massive neutrinos there exists a set of mass eigenstates denoted by $\nu_{i}$ which describe the evolution of neutrinos in space and time. Interactions of neutrinos with matter are described by flavour eigenstates $\nu_{\alpha}$. The mixing between states occurs with a rotation matrix $U$, the PMNS matrix, which satisfies $U^{\dagger} U=\mathbb{1}$. The following mathematical derivation is generalised for $N$ neutrinos.

A source of neutrinos will produce a neutrino at a time $t=0$ in a weak flavour 
eigenstate $\left|\nu_{\alpha}(t=0)\right\rangle$ that is a superposition of mass eigenstates $\left|\nu_{i}\right\rangle$ :

$$
\left|\nu_{\alpha}(0)\right\rangle=\sum_{i} U_{\alpha i}^{*}\left|\nu_{i}\right\rangle
$$

The mass eigenstates will evolve with time as they propagate,

$$
\left|\nu_{\alpha}(t)\right\rangle=\sum_{i} U_{\alpha i}^{*} e^{-i p_{i} \cdot x}\left|\nu_{i}\right\rangle
$$

where $x$ is the four-vector position of the neutrino and $p_{i}$ is the four-vector momentum of the mass state $i$. At time $t$ the neutrino is observed via the weak interaction in a detector and the wave function will become a weak eigenstate of flavour, $\nu_{\beta}$ :

$$
\left\langle\nu_{\beta}\right|=\sum_{j} U_{\beta j}\left\langle\nu_{j}\right|
$$

The amplitude for this observation is given by

$$
\begin{aligned}
\left\langle\nu_{\beta} \mid \nu_{\alpha}(t)\right\rangle & =\left(\sum_{j} U_{\beta j}\left\langle\nu_{j}\right|\right)\left(\sum_{i} U_{\alpha i}^{*} e^{-i p_{i} \cdot x}\left|\nu_{i}\right\rangle\right) \\
& =\sum_{i} \sum_{j} U_{\beta j} U_{\alpha i}^{*} \underbrace{\left\langle\nu_{j} \mid \nu_{i}\right\rangle}_{\delta_{i j}} e^{-i p_{i} \cdot x} \\
& =\sum_{i} U_{\beta i} U_{\alpha i}^{*} e^{-i p_{i} \cdot x},
\end{aligned}
$$

where $\delta_{i j}$ is the Kronecker delta.

By denoting $m_{i}$ and $E_{i}$ as the mass and energy, respectively, of the $i^{\text {th }}$ mass eigenstate we can express the argument of the exponential as

$$
p_{i} \cdot x=E_{i} t-\vec{p} \cdot \vec{x},
$$

where we make the approximation that all mass eigenstates have the same threevector momentum $\vec{p}$. A more rigorous approach can be taken by treating the 
neutrinos as wave packets; this eliminates the need for this momentum assumption and has been shown to lead to the same result $[55,56]$.

By expressing the energy as $E_{i}=\left(|\vec{p}|^{2}+m_{i}^{2}\right)^{\frac{1}{2}}$, assuming $m_{i} \ll E_{i}$ and using natural units, one can say $t=L$ and $\vec{p} \cdot \vec{x}=|\vec{p}| L$ (where $L$ is the distance travelled). We can perform a binomial expansion on the expression for the energy, so that equation 2.10 can be expressed as

$$
\begin{aligned}
p \cdot x & =|\vec{p}| L\left(1+\frac{m_{i}^{2}}{2|\vec{p}|^{2}}\right)-|\vec{p}| L \\
& =\frac{m_{i}^{2} L}{2 E}
\end{aligned}
$$

where $|\vec{p}| \approx E$ and $E$ is the average over $E_{i}$. Therefore we have

$$
\left\langle\nu_{\beta} \mid \nu_{\alpha}(L)\right\rangle=\sum_{i} U_{\beta i} U_{\alpha i}^{*} e^{-\frac{i m_{i}^{2} L}{2 E}}
$$

We can now compute the probability of measuring the original $\nu_{\alpha}$ as being of flavour $\nu_{\beta}$ by finding the square of the amplitude:

$$
\begin{aligned}
P\left(\nu_{\alpha} \rightarrow \nu_{\beta}\right)= & \left|\left\langle\nu_{\beta} \mid \nu_{\alpha}(L)\right\rangle\right|^{2} \\
= & \left(\sum_{i} U_{\beta i}^{*} U_{\alpha i} e^{-\frac{i m_{i}^{2} L}{2 E}}\right)\left(\sum_{j} U_{\beta j} U_{\alpha j}^{*} e^{\frac{i m_{j}^{2} L}{2 E}}\right) \\
= & \sum_{i} \sum_{j} U_{\beta i}^{*} U_{\alpha i} U_{\beta j} U_{\alpha j}^{*} e^{-\frac{i L}{2 E}\left(m_{i}^{2}-m_{j}^{2}\right)} \\
= & \sum_{i} \sum_{j} U_{\beta i}^{*} U_{\alpha i} U_{\beta j} U_{\alpha j}^{*} e^{-\frac{i \Delta m_{i j}^{2} L}{2 E}} \\
& \quad+\left[\sum_{i} \sum_{j} U_{\beta j}^{*} U_{\beta i} U_{\alpha i}^{*} U_{\alpha j}-\sum_{i} \sum_{j} U_{\beta j}^{*} U_{\beta i} U_{\alpha i}^{*} U_{\alpha j}\right],
\end{aligned}
$$

where $\Delta m_{i j}^{2}=\left(m_{i}^{2}-m_{j}^{2}\right)$. The last two terms sum to zero and are included so 
that equation 2.14 can be rearranged:

$$
P\left(\nu_{\alpha} \rightarrow \nu_{\beta}\right)=\sum_{i} \sum_{j} \underbrace{U_{\beta j}^{*} U_{\beta i} U_{\alpha i}^{*} U_{\alpha j}\left(e^{-i \frac{\Delta m_{i j}^{2} L}{2 E}}-1\right)}_{A_{i j}}+\sum_{i} \sum_{j} U_{\beta j}^{*} U_{\beta i} U_{\alpha i}^{*} U_{\alpha j} .
$$

Recalling the fact that $U^{\dagger}=\left(U^{*}\right)^{\top}$, the second term in equation 8.1 can be simplified as follows,

$$
\begin{aligned}
\sum_{i} \sum_{j} U_{\beta j}^{*} U_{\beta i} U_{\alpha i}^{*} U_{\alpha j} & =\sum_{i} U_{\beta i} U_{\alpha i}^{*} \sum_{j} U_{\beta j}^{*} U_{\alpha j} \\
& =\sum_{i} U_{\beta i} U_{i \alpha}^{\dagger} \sum_{j} U_{j \beta}^{\dagger} U_{\alpha j} \\
& =\delta_{\alpha \beta} .
\end{aligned}
$$

Looking at $A_{i j}$ we can see that $A_{i i}=0$ due to $\Delta m_{i i}^{2}=0$ and that $A_{i j}=A_{j i}^{*}$ (because of the minus sign due to $\Delta m_{i j}^{2}=-\Delta m_{j i}^{2}$ ), meaning that $2 \mathfrak{R e}\left[A_{i j}\right]=$ $A_{i j}+A_{j i}^{*}$. These facts mean that we can remove the $i=j$ terms and group the pairs together:

$$
P\left(\nu_{\alpha} \rightarrow \nu_{\beta}\right)=\delta_{\alpha \beta}+2 \sum_{i(>j)} \sum_{j} \mathfrak{R e}\left(U_{\beta j}^{*} U_{\beta i} U_{\alpha i}^{*} U_{\alpha j}\left(e^{-i \frac{\Delta m_{i j}^{2} L}{2 E}}-1\right)\right)
$$

Using Euler's identity,

$$
e^{-i \frac{\Delta m_{i j}^{2} L}{2 E}}=\cos \left(\frac{\Delta m_{i j}^{2}}{2 E} L\right)-i \sin \left(\frac{\Delta m_{i j}^{2} L}{2 E}\right)
$$

and the identity

$$
1-\cos \left(\frac{\Delta m_{i j}^{2} L}{2 E}\right)=2 \sin ^{2}\left(\frac{\Delta m_{i j}^{2} L}{4 E}\right)
$$


equation 8.2 can be expressed as

$$
\begin{aligned}
P\left(\nu_{\alpha} \rightarrow \nu_{\beta}\right)= & \delta_{\alpha \beta} \\
& +2 \sum_{i(>j)} \sum_{j} \mathfrak{I m}\left[U_{\beta j}^{*} U_{\beta i} U_{\alpha i}^{*} U_{\alpha j} \sin \left(\frac{\Delta m_{i j}^{2} L}{2 E}\right)\right] \\
& -4 \sum_{i(>j)} \sum_{j} \mathfrak{R e}\left[U_{\beta j}^{*} U_{\beta i} U_{\alpha i}^{*} U_{\alpha j} \sin ^{2}\left(\frac{\Delta m_{i j}^{2} L}{4 E}\right)\right] .
\end{aligned}
$$

Equation 2.19 is a general expression for $N$ neutrinos that experience oscillation in a vacuum. When neutrinos traverse through matter the eigenstates become modified due to the MSW effect $[57,58]$. The effect is most significant when observing solar neutrinos or electron neutrino appearance over long baselines. The analysis presented in this thesis is insensitive to matter effects [59], as will be discussed further in chapter 7 . The approximation of neutrinos travelling through a vacuum is therefore used throughout.

\subsubsection{Three-Flavour Model}

In the case of three flavour and mass states, they can be related by

$$
\left(\begin{array}{c}
\nu_{e} \\
\nu_{\mu} \\
\nu_{\tau}
\end{array}\right)=U^{*}\left(\begin{array}{c}
\nu_{1} \\
\nu_{2} \\
\nu_{3}
\end{array}\right) .
$$

$U$ can be parametrised by three mixing angles $\left(\theta_{12}, \theta_{23}, \theta_{13}\right)$ and a phase $\delta$ : 


$$
\begin{aligned}
U & =\left(\begin{array}{ccc}
U_{e 1} & U_{e 2} & U_{e 3} \\
U_{\mu 1} & U_{\mu 2} & U_{\mu 3} \\
U_{\tau 1} & U_{\tau 2} & U_{\tau 3}
\end{array}\right) \\
& =\underbrace{\left(\begin{array}{ccc}
c_{12} & s_{12} & 0 \\
-s_{12} & c_{12} & 0 \\
0 & 0 & 1
\end{array}\right)}_{\text {solar }}\left(\begin{array}{ccc}
c_{13} & 0 & s_{13} e^{-i \delta} \\
0 & 1 & 0 \\
-s_{13} e^{i \delta} & 0 & c_{13}
\end{array}\right) \underbrace{\left(\begin{array}{ccc}
1 & 0 & 0 \\
0 & c_{23} & s_{23} \\
0 & -s_{23} & c_{23}
\end{array}\right)} \\
& =\left(\begin{array}{ccc}
c_{12} c_{13} \\
-s_{12} c_{23}-c_{12} s_{23} s_{13} e^{i \delta} & c_{12} c_{23}-s_{12} s_{23} s_{13} e^{i \delta} & s_{23} c_{13} \\
s_{12} s_{23}-c_{12} c_{23} s_{13} e^{i \delta} & -c_{12} s_{23}-s_{12} c_{23} s_{13} e^{i \delta} & c_{23} c_{13}
\end{array}\right),
\end{aligned}
$$

where $c_{i j} \equiv \cos \theta_{i j}$ and $s_{i j} \equiv \sin \theta_{i j}$. The solar term controls the amount of electron neutrino mixing between states $\nu_{1}$ and $\nu_{2}$ and is governed by the value of $\theta_{12}$. The atmospheric term controls how the $\nu_{\mu}$ and $\nu_{\tau}$ flavour mixes and is governed by the mixing angle $\theta_{23}$. The middle term contains the unmeasured CP violating phase, as well as $\theta_{13}$ which determines the amount of $\nu_{e}$ mixing into the third mass state $\nu_{3}$. Within a three-flavour framework, the energy-dependence of neutrino oscillation is driven by the mass splittings $\Delta m_{32}^{2}$ and $\Delta m_{21}^{2}$, where $\Delta m_{31}^{2}=\Delta m_{32}^{2}+\Delta m_{21}^{2}$.

A summary of current measurements of the mass splitting and mixing angles from a three-flavour global fit to experimental data are shown in table 2.1. However, there exist a number of unknowns that current and future experiments are attempting to address as well as providing precision measurements of all parameters:

- The value of $\delta_{\mathrm{CP}}$ : Analogous to the quark sector, neutrino oscillations are parametrised with a CP-violating phase. This parameter has proven diffi- 


\begin{tabular}{|c|c|c|}
\hline Parameter & $\Delta m^{2}$ Ordering & Best Fit $( \pm 1 \sigma)$ \\
\hline \hline$\Delta m_{21}^{2} / 10^{-5} \mathrm{eV}^{2}$ & Both & $7.54_{-0.22}^{+0.26}$ \\
$\sin ^{2} \theta_{12} / 10^{-1}$ & Both & $3.08_{-0.17}^{+0.17}$ \\
\hline$\Delta m^{2} / 10^{-3} \mathrm{eV}^{2}$ & Normal & $2.43_{-0.06}^{+0.06}$ \\
$\Delta m^{2} / 10^{-3} \mathrm{eV}^{2}$ & Inverted & $-2.38_{-0.06}^{+0.06}$ \\
\hline $\sin ^{2} \theta_{13} / 10^{-2}$ & Normal & $2.34_{-0.19}^{+0.20}$ \\
$\sin ^{2} \theta_{13} / 10^{-2}$ & Inverted & $2.40_{-0.22}^{+0.19}$ \\
\hline $\sin ^{2} \theta_{23} / 10^{-1}$ & Normal & $4.37_{-0.23}^{+0.33}$ \\
$\sin ^{2} \theta_{23} / 10^{-1}$ & Inverted & $4.55_{-0.31}^{+1.39}$ \\
\hline$\delta_{C P} / \pi$ & Normal & $1.39_{-0.27}^{+0.38}$ \\
$\delta_{C P} / \pi$ & Inverted & $1.31_{-0.33}^{+0.29}$ \\
\hline
\end{tabular}

Table 2.1: Values of oscillation variables from a three-flavour global fit to experimental data. Note that $\Delta m^{2} \equiv m_{3}^{2}-\left(m_{2}^{2}+m_{1}^{2}\right) / 2$. Values taken from [60].

cult to measure as it primarily effects the small amount of electron-neutrino appearance in long-baseline experiments.

- The neutrino mass ordering: For the three-flavour case the differences in mass between the eigenstates $\nu_{1}, \nu_{2}$ and $\nu_{3}$ govern the energy-dependence of neutrino oscillations. The sign of $\Delta m_{21}^{2}$ has been measured to be positive, however the sign of the mass splitting $\Delta m_{32}^{2}$, commonly measured by observing atmospheric neutrino oscillations, has not been determined. Muon-neutrino disappearance measurements have yielded a value but are not sensitive to the sign. Two possibilities of the mass ordering exist, the normal ordering $\left(\nu_{1}<\nu_{2}<\nu_{3}\right)$ or the inverted ordering $\left(\nu_{3}<\nu_{1}<\nu_{2}\right)$.

- The octant of $\theta_{23}$ : It is not clear if $\theta_{23}$ is smaller or larger than $\pi / 4$ (or exactly $\pi / 4$ ) due to the leading term in the muon survival probability for the amplitude of neutrino mixing being $\sin ^{2}\left(2 \theta_{23}\right)$. Precision measurements, including electron-neutrino appearance measurements, are required to determine this. 


\subsection{Sterile Neutrinos}

The three-flavour neutrino oscillation model has been experimentally well-established with a majority of results agreeing with the model. However, a small subset of anomalous results have arisen which, amongst possible explanations, can be reconciled by the addition of one or more pairs of neutrino mass and flavour states.

By introducing additional neutrino flavour and mass states one has to remember the results from the measurement of the $Z$ boson decay width from LEP, shown in figure 2.1. Additional neutrinos (with a mass less than half the mass of the $Z$ boson) must therefore not interact through the weak interaction. These beyond-the-Standard-Model particles are called sterile neutrinos, a terminology first introduced by Pontecorvo in 1969 [61]. The following anomalous results are the main catalysts for motivating the work presented in this thesis. The list of possible hints for sterile neutrinos is not entirely covered in this thesis; a more complete review of experimental indications of sterile neutrinos can be found in [62].

\subsubsection{Electron Neutrino Appearance Anomalies}

There have been anomalous results in short-baseline neutrino oscillation experiments (an $L / E \sim 1 \mathrm{~km} / \mathrm{GeV}$ ). The two most famous examples looked for the presence of electron anti-neutrinos in a beam of muon neutrinos.

\subsubsection{LSND}

The Liquid Scintillator Neutrino Detector (LSND) was a short-baseline neutrino oscillation experiment built to search for $\bar{\nu}_{\mu} \rightarrow \bar{\nu}_{e}$. The $\bar{\nu}_{\mu}$ neutrino beam was produced at the Los Alamos Neutron Science Center (LANSCE) by colliding $789 \mathrm{MeV}$ protons onto a target, producing a large number of pions. The open space around the target was short compared to the pion decay length allowing for 
the pions and muons to decay at rest. The $\pi^{-}$were absorbed by the surrounding material whilst the $\pi^{+}$decayed primarily into muons which decay into $\bar{\nu}_{\mu}$ with energies ranging from $20-100 \mathrm{MeV}$ with a peak around $40 \mathrm{MeV}$, with over $95 \%$ of these neutrinos resulting from decay at rest and the remainder from decay in flight.

The LSND detector was a cylindrical tank $8.3 \mathrm{~m}$ long by $5.7 \mathrm{~m}$ in diameter situated $30 \mathrm{~m}$ from the target. The tank was filled with 167 metric tons of liquid scintillator with the inside covered with 1220 8-inch Hamamatsu photomultiplier tubes. The $\bar{\nu}_{e}$ events were identified through the interaction $\bar{\nu}+p \rightarrow e^{+}+n$; the signal from positron annihilation in coincidence with the $2.2 \mathrm{MeV} \gamma$ released by the capture of the neutron by a free proton was used.

LSND took data from 1993 up until 1998 with the results showing the size of the $\bar{\nu}_{e}$ excess of $87.9 \pm 22.4$ (stat.) \pm 6.0 (syst.) events in the energy range of 20-75 MeV [63]. This is a deviation from the three-flavour neutrino oscillation model of $3.8 \sigma$. To account for this excess due to neutrino oscillation one would require a new mass splitting, $\Delta m^{2}$, of the order $0.2-10 \mathrm{eV}^{2}$, which is incompatible with the two measured mass splittings described by the three-flavour model that are shown in table 2.1 .

\subsubsection{MiniBooNE}

The MiniBooNE experiment was designed to measure $\nu_{\mu} \rightarrow \nu_{e}$ and $\bar{\nu}_{\mu} \rightarrow \bar{\nu}_{e}$; an independent check of the LSND evidence that indicates neutrino oscillations with a mass splitting at the $1 \mathrm{eV}^{2}$ scale.

The MiniBooNE experiment consisted of a single spherical detector, $12.2 \mathrm{~m}$ in diameter, filled with 800 tons of mineral oil $\left(\mathrm{CH}_{2}\right)$. With a baseline of $500 \mathrm{~m}$, MiniBooNE was designed to have the same $L / E$ as the LSND experiment. MiniBooNE identified charged current quasi-elastic $\nu_{e}$ and $\bar{\nu}_{e}$ interactions in the detector by collecting the Cherenkov light produced by the scintillation of the final 
state charged particles. The light was detected by 1580 8-inch PMTs situated within the interior of the detector.

The $\nu_{\mu}$ or $\bar{\nu}_{\mu}$ beam was produced by taking $8 \mathrm{GeV}$ protons from the Fermilab Booster, and directing them into a magnetic focusing horn where they struck a Beryllium target. The proton-Beryllium interactions resulted in a plethora of charged mesons which decayed in flight along an air-filled decay region. Following the decay region was an absorber, put in place to stop any undecayed mesons and muons. The remaining neutrinos travelled towards the MiniBooNE detector resulting in a neutrino beam peaked at around $500 \mathrm{MeV}$. The polarity of the horns could be reversed to produce a beam of $\nu_{\mu}$ or $\bar{\nu}_{\mu}$.

MiniBooNE performed two analyses, for neutrino and anti-neutrino beams. A combined fit to both neutrino and anti-neutrino data for the reconstructed neutrino energy range of $200-1250 \mathrm{GeV}$ gives an excess of $240.3 \pm 34.5$ (stat.) \pm 52.6(syst.) electron events, a significance of $3.8 \sigma$ [64]. The results from MiniBooNE support the evidence seen by LSND with an excess that could be explained by a mass splitting incompatible with the three-flavour model.

\subsubsection{Reactor Anomalies}

Reactor neutrino experiments have helped to experimentally establish direct evidence of neutrino oscillations. A neutrino reactor experiment relies on knowing the $\bar{\nu}_{e}$ flux from the reactor in order to calculate a predicted event rate of $\bar{\nu}_{e}$ events in the detector that can be compared to data. By calculating the ratio, $R$, of observed events to the predicted number, one can observe a deficit of events due to neutrino oscillation. This is complimentary to fits to the energy distribution of $\bar{\nu}_{e}$ events (a shape analysis).

Up until 2011 the $\bar{\nu}_{e}$ flux had been estimated from measurements of the total electron spectra associated with the beta decays from fission products of ${ }^{235} \mathrm{U}$, ${ }^{239} \mathrm{Pu}$ and ${ }^{241} \mathrm{Pu}$ using a set of 30 effective beta-decay chains [65]. More recently 
the estimation of the $\bar{\nu}_{e}$ flux has been revisited [66], taking into account all betadecay branches from current nuclear databases [67].

The new calculation of the $\bar{\nu}_{e}$ flux led to a $+3 \%$ shift in the normalisation of the $\bar{\nu}_{e}$ flux from the fission products of ${ }^{235} \mathrm{U},{ }^{239} \mathrm{Pu}$ and ${ }^{241} \mathrm{Pu}$. The effect on past experimental data results in a shift for the $R$ values. Figure 2.4 shows the $R$ values for various reactor experiments. The red line indicates the expected $R$ as a function of baseline for the three-flavour neutrino oscillation case. For reactors with a baseline less than $1 \mathrm{~km}$ in the three-flavour case, there should be no neutrino oscillation and one would expect $R$ to equal unity. The blue line shows the value of $R$ if an additional neutrino, a sterile neutrino, is considered. This appears to be in better agreement with the data. This is called the "reactor antineutrino anomaly".

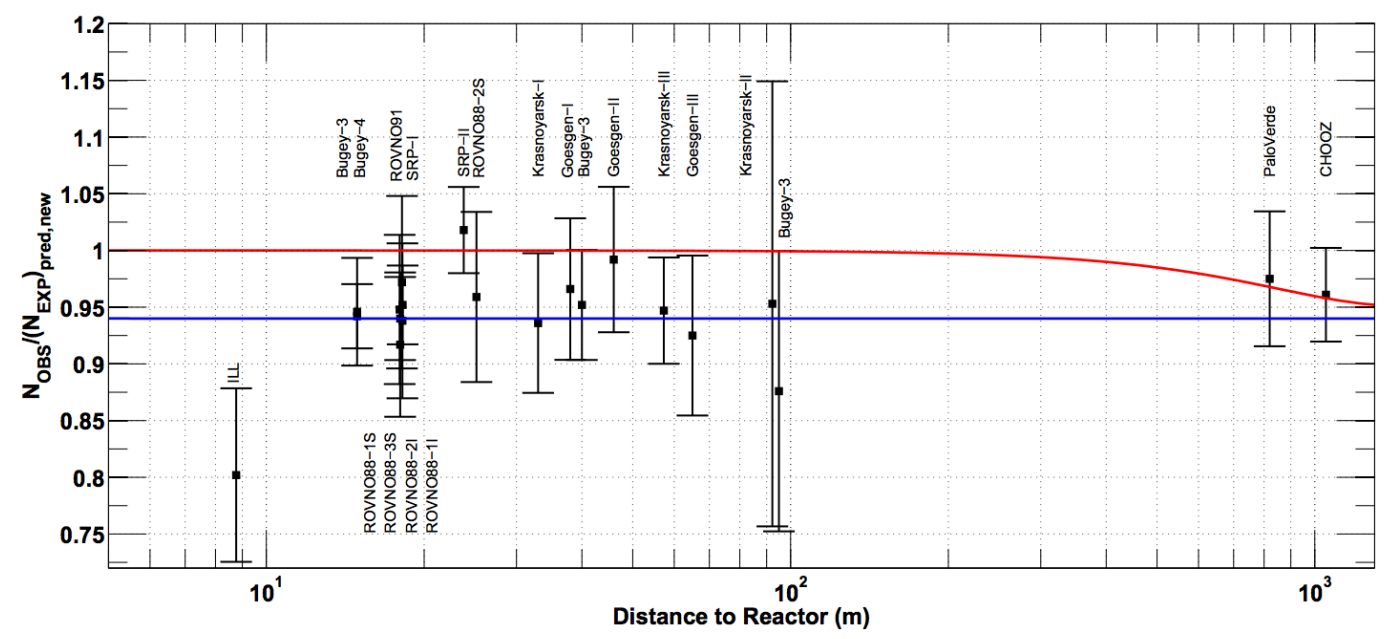

Figure 2.4: The short-baseline reactor-antineutrino anomaly. The black data points are the $R$ values for different reactor experiments. The red line indicates the expected $R$ as a function of baseline for the three-flavour neutrino oscillation case and the blue line shows the value of $R$ if an additional neutrino, a sterile neutrino with a mass splitting $\Delta m^{2}$ on the scale of $1 \mathrm{eV}^{2}$, is considered. Graph taken from [66]. 


\subsubsection{Gallium Anomalies}

The solar neutrino experiments GALLEX and SAGE, which were mentioned for their work on the solar neutrino problem, have also observed anomalous results that could be explained by a model with sterile neutrinos.

The SAGE experiment, concerned about its measurement of the neutrino capture rate compared to solar models, performed two separate tests to determine its response to low-energy neutrinos $[68,69]$. To produce an intense artificial source of $\nu_{e}$ neutrinos two different radioactive sources were used. A ${ }^{51} \mathrm{Cr}$ source and an ${ }^{37}$ Ar source were used that both emitted mono-energetic electron neutrinos.

The neutrinos emitted by the radioactive sources were detected by the same method as for the solar neutrino analyses through $\nu_{e}+{ }^{71} \mathrm{Ga} \rightarrow e^{-}+{ }^{71} \mathrm{Ge}$. The cross-section for the production of ${ }^{71} \mathrm{Ge}$ was measured for each source, and the ratios of the cross-section to the theoretical cross-section, $R_{\mathrm{S} 1}^{\mathrm{Cr}}$ and $R_{\mathrm{S} 2}^{\mathrm{Ar}}$, for the SAGE experiment were found to be

$$
\begin{aligned}
& R_{\mathrm{S} 1}^{\mathrm{Cr}}=0.95 \pm 0.12, \\
& R_{\mathrm{S} 2}^{\mathrm{Ar}}=0.791_{-0.078}^{+0.084}
\end{aligned}
$$

For the same reasons the GALLEX experiment performed a similar study using two sources of ${ }^{51} \mathrm{Cr}[70,71]$. The two sources were installed for $3-4$ months in the centre of the GALLEX detector with the experimental conditions kept as close as possible as for the previous solar neutrino analyses. For the two ${ }^{51} \mathrm{Cr}$ sources the ratios of the measured cross-section to the theoretical cross-section, $R_{\mathrm{G} 1}^{\mathrm{Cr}}$ and $R_{\mathrm{G} 2}^{\mathrm{Cr}}$ were found to be

$$
\begin{aligned}
& R_{\mathrm{G} 1}^{\mathrm{Cr}}=0.953 \pm 0.11, \\
& R_{\mathrm{G} 2}^{\mathrm{Cr}}=0.812_{-0.11}^{+0.10} .
\end{aligned}
$$


A global statistical significance of the anomalous deficit of electron neutrinos measured using these radioactive sources in GALLEX and SAGE was performed and there was found to be a $3 \sigma$ deviation from the calculated theoretical cross section [72]. This is known as the "gallium anomaly". One possible explanation for these results is to interpret this as hints for observing $\nu_{e}$ disappearance due to the existence of sterile neutrinos that oscillate with active neutrino flavours.

\subsubsection{The Sterile Neutrino Model}

The simplest sterile neutrino model is the $3+1$ sterile neutrino phenomenological model, that is, an additional neutrino being considered of flavour $\nu_{s}$. This model extends the PMNS matrix to four dimensions, introducing additional matrix elements as seen below:

$$
U=\left(\begin{array}{cccc}
U_{e 1} & U_{e 2} & U_{e 3} & U_{e 4} \\
U_{\mu 1} & U_{\mu 2} & U_{\mu 3} & U_{\mu 4} \\
U_{\tau 1} & U_{\tau 2} & U_{\tau 3} & U_{\tau 4} \\
U_{s 1} & U_{s 2} & U_{s 3} & U_{s 4}
\end{array}\right)
$$

This gives rise to an extra mass splitting $\Delta m_{41}^{2}$, three additional mixing angles $\theta_{14}, \theta_{24}$, and $\theta_{34}$, and two additional CP violating phases $\delta_{14}$ and $\delta_{24}$. Note that in this notation the previous three-flavour CP violating phase becomes $\delta_{C P} \rightarrow \delta_{13}$.

The oscillation probabilities for neutrino disappearance and appearance become altered from the three-flavour model. The mixing angle $\theta_{14}$ governs the oscillation between electron and sterile flavour states, which plays an important role in explaining the reactor and gallium neutrino anomalies. The angle $\theta_{24}$

governs the oscillation between muon and sterile neutrino flavour states and $\theta_{34}$ determines the mixing between tau and sterile neutrino flavour states.

Appendix A presents the matrix elements in terms of the mixing angles along with derivations of the oscillation probabilities. A combination between $\theta_{14}$ and 
$\theta_{24}$ is required to to explain the excess of electron neutrinos from a muon neutrino beam for the LSND and MiniBooNE appearance experiments, $P\left(\nu_{\mu} \rightarrow \nu_{e}\right)$. Adding a sterile neutrino at the $1 \mathrm{eV}^{2}$ scale, the expression for $P\left(\nu_{\mu} \rightarrow \nu_{e}\right)$ can be expressed as

$$
P\left(\nu_{\mu} \rightarrow \nu_{e}\right) \approx 4\left|U_{e 4}\right|^{2}\left|U_{\mu 4}\right|^{2} \sin ^{2} \Delta_{41}=\sin ^{2} 2 \theta_{\mu e} \sin ^{2} \Delta_{41},
$$

where $\sin ^{2} 2 \theta_{\mu e}=4\left|U_{e 4}\right|^{2}\left|U_{\mu 4}\right|^{2}=\sin ^{2} 2 \theta_{14} \sin ^{2} \theta_{24}$. For convenience one can redefine $\frac{\Delta m_{i j}^{2} L}{4 E}=\Delta_{i j}$, with the terms $\Delta_{31}$ and $\Delta_{21}$ being neglected as they become small over short baselines. Using this model the results from the LSND and MiniBooNE experiments are shown in figure 2.5 as $90 \%$ confidence intervals for the $3+1$ sterile neutrino parameter space using the effective mixing angle $\theta_{\mu e}$ and the sterile mass splitting $\Delta m_{41}^{2}$.

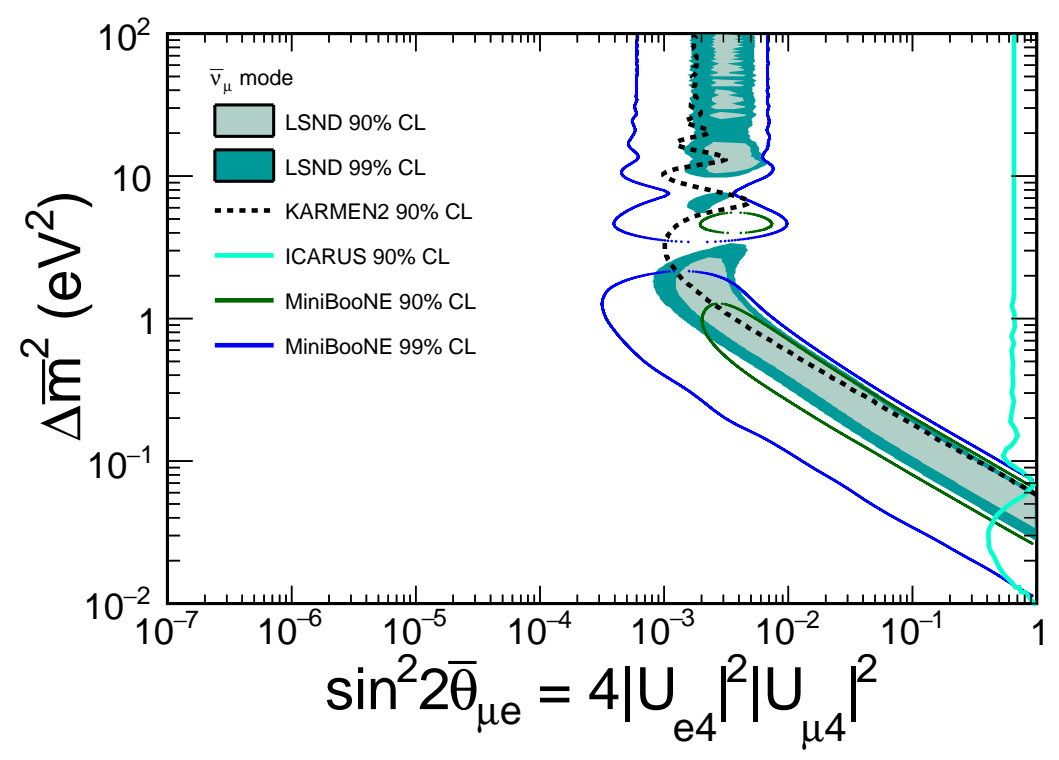

Figure 2.5: The LSND and MiniBooNE 90\% and 99\% allowed region for sterile anti-neutrinos. The figure also shows exclusion regions for Karmen2 [73] and ICARUS [74] experiments.

The same $3+1$ sterile neutrino phenomenological model is used to analyse the MINOS data in this thesis, presented in chapter 7 . The region of $L / E$ probed 
by the MINOS detectors covers the region of allowed parameter space for mass splittings over the range $10^{-4}-10^{2} \mathrm{eV}^{2}$. The muon disappearance channel used by the MINOS experiment gives sensitivity to the sterile mixing angle $\theta_{24}$. A combination is then performed with the neutrino reactor experiment, Bugey-3, which looked for electron anti-neutrino disappearance that is sensitive to $\theta_{14}$. This combination allows for a direct limit to be placed on the quantity $\sin ^{2} 2 \theta_{14} \sin ^{2} \theta_{24}$ which is the $\sin ^{2} 2 \theta_{\mu e}$ quantity in which LSND and MiniBooNE report their allowed regions. 


\section{Chapter 3}

\section{The MINOS and MINOS+ Experiments}

This chapter gives an overview of the MINOS and MINOS+ experiments followed by descriptions of the NuMI beam-line, the NuMI targets and the MINOS detectors. The last section provides information on the type of neutrino interactions within the detectors.

\subsection{MINOS}

MINOS $[75,76]$ (the Main Injection Neutrino Oscillation Search) is a long-baseline neutrino oscillation experiment primarily designed to measure $\nu_{\mu}$ and $\bar{\nu}_{\mu}$ disappearance. MINOS uses a beam of predominantly muon neutrinos provided by the NuMI (Neutrinos at the Main Injector) beam-line facility [77] at Fermilab located near Chicago, IL, USA. The MINOS experiment is a two-detector experiment. The Near Detector (ND) is situated $1.04 \mathrm{~km}$ downstream from the neutrino production target and makes precise measurements of the neutrino beam before neutrino oscillations have occurred. The Far Detector (FD) is $735 \mathrm{~km}$ downstream from the neutrino production target and is located in the Soudan Underground Laboratory, northern Minnesota. The FD measures the neutrino beam once os- 
cillations have occurred. It is the comparison between both detectors that allows for the inference of neutrino oscillation by observing a deficit of muon-flavour neutrinos (or an appearance of electron neutrinos). The setup of the MINOS experiment is shown in figure 3.1 .

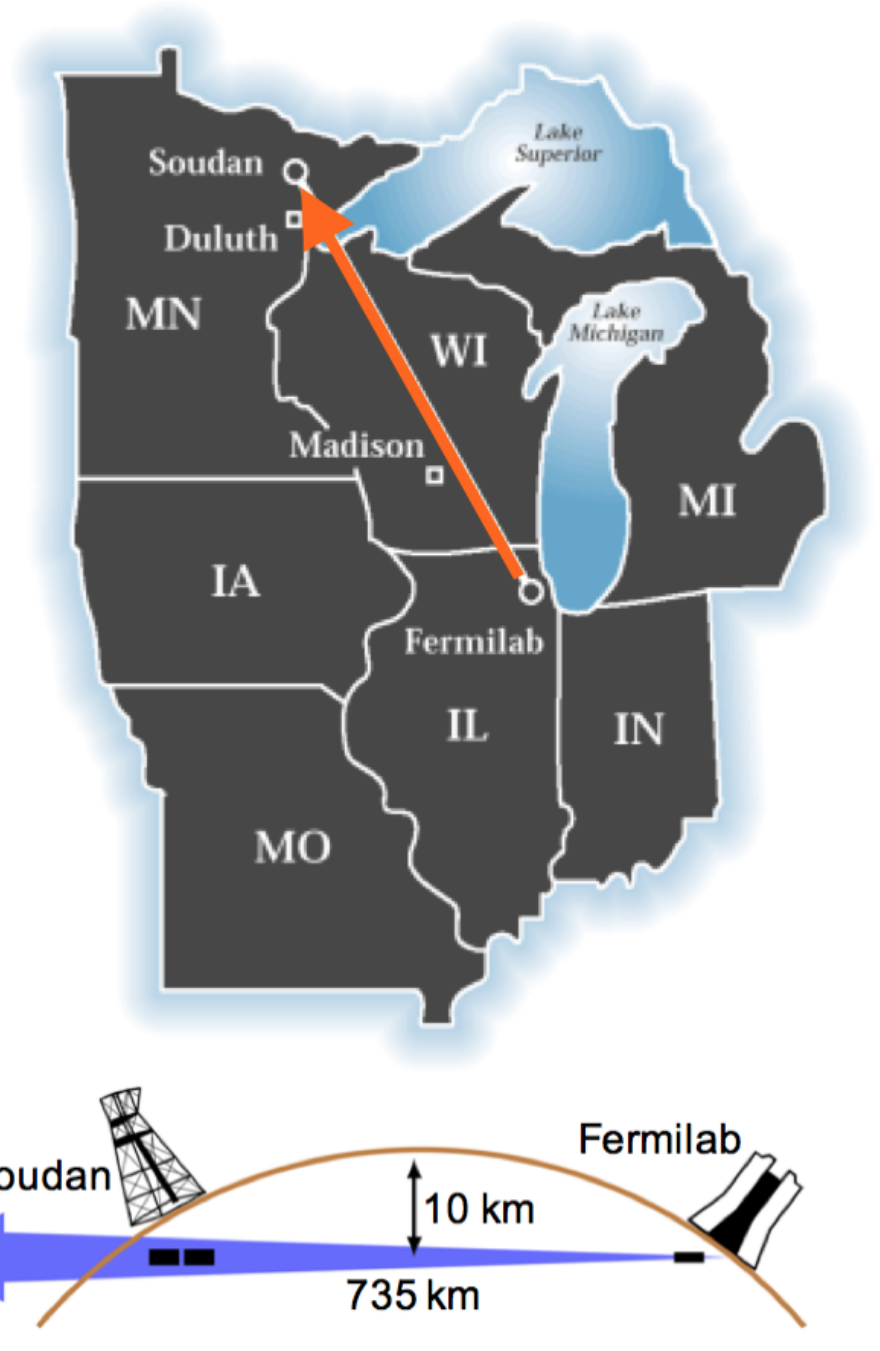

Figure 3.1: The MINOS experiment. A beam of muon-type neutrinos is created at the NuMI facility. The neutrinos pass through the Near Detector located at Fermilab and the Far Detector located in the Soudan Mine in northern Minnesota. The baseline is $735 \mathrm{~km}$.

MINOS began taking data in March 2005 and continued up until April 2012, collecting data in both $\nu_{\mu}$ and $\bar{\nu}_{\mu}$ beam modes (explained in section 3.3). A break-down of the beam conditions for this data-taking period can be seen in 


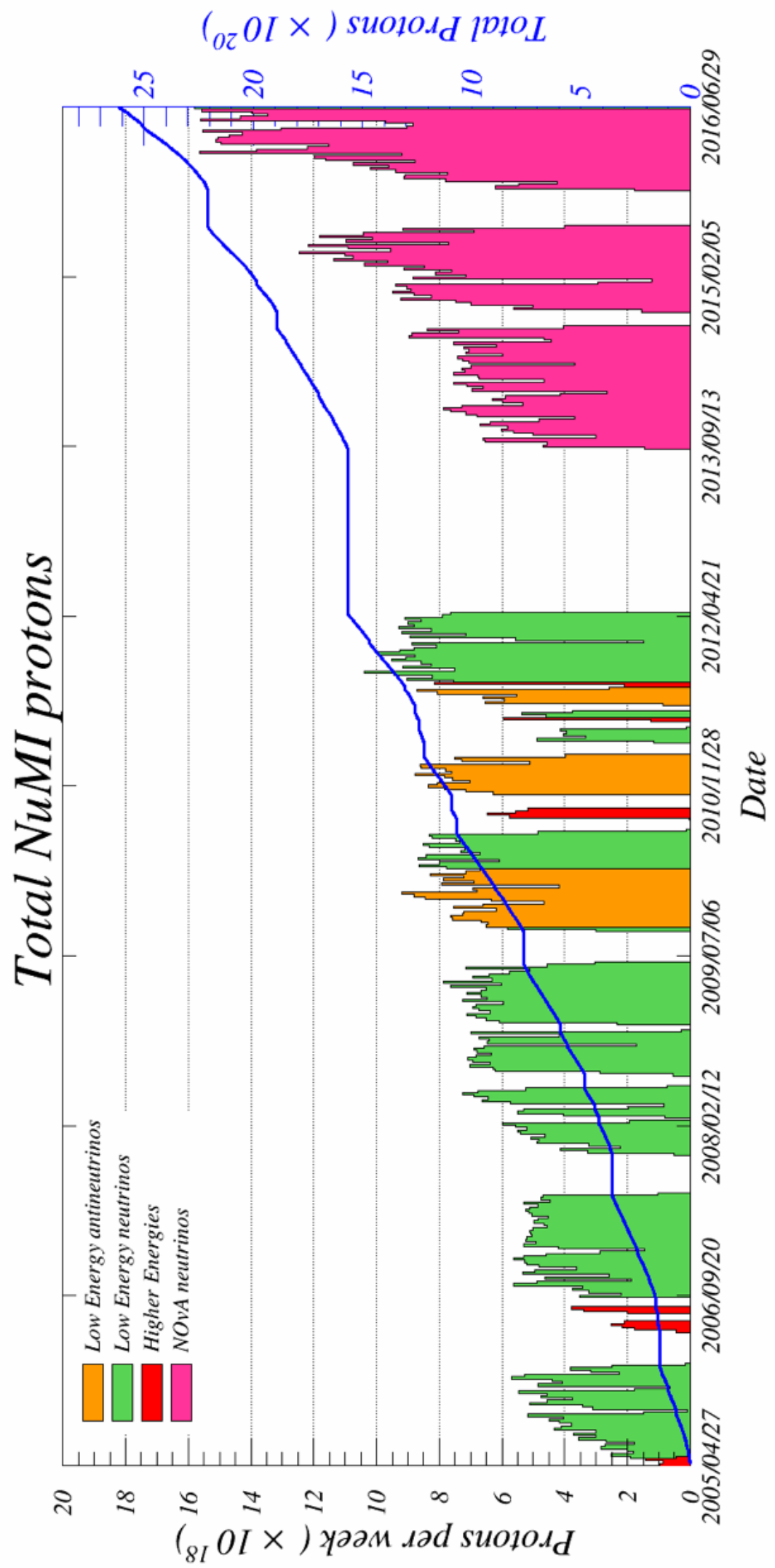

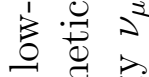

동

키 임

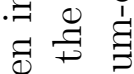

牙宁 要

की कृ

宩

गु

荡

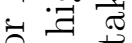

용

营

응

\pm ?

拧

苏蛅

$\sum \xi$

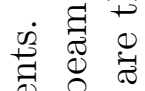

寻要

讨

ब웡

$+\bar{\beta}$ 。

次

Z

$\left.\sum.\right]$

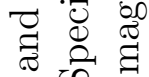

की

$8 \infty_{-0}$

$\sum$ त्ष

응

궁

용

茟

클

㖊

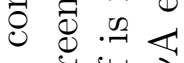

毒 50 出

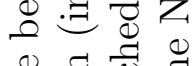

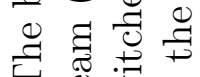

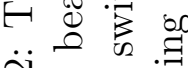

iे $)^{2}$

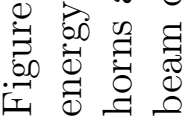


figure 3.2. The majority of the data was taken in the low-energy configuration with a beam composed predominantly of $\nu_{\mu}$ with an energy spectrum peaked at around $3 \mathrm{GeV}$. MINOS also uses an atmospheric neutrino sample of $37.88 \mathrm{kt}-\mathrm{yr}$ at the Far Detector, which is $705 \mathrm{~m}$ underground (2070 mwe) [78].
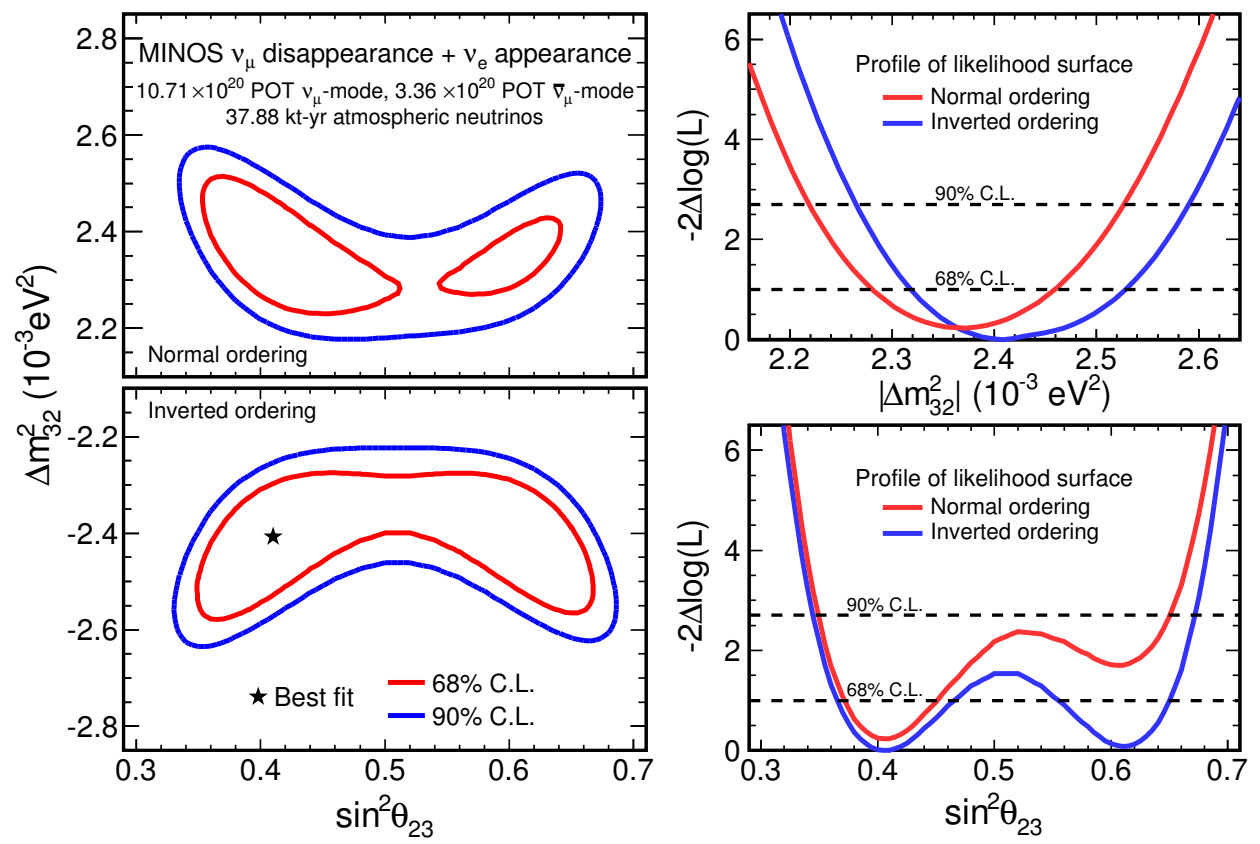

Figure 3.3: The left panels show the $68 \%$ and $90 \%$ confidence limits on $\Delta m_{32}^{2}$ and $\sin ^{2} \theta_{23}$ for the normal ordering (top) and inverted ordering (bottom). At each point in this parameter space, the likelihood function is maximised with respect to $\sin ^{2} \theta_{13}, \delta_{c p}$ and the systematic parameters constrained in the fit. The likelihood surface is calculated relative to the overall best fit, which is indicated by the star. The right panels show the 1D likelihood profiles as a function of $\Delta m_{32}^{2}$ and $\sin ^{2} \theta_{23}$ for each mass ordering. The horizontal dotted lines indicate the $68 \%$ and $90 \%$ confidence limits. Figure taken from [79].

Using the low-energy dataset and the atmospheric neutrino sample a previous MINOS analysis measured the most precise value of $\Delta m_{32}^{2}$ to date along with a competitive measurement of $\theta_{23}$ [79]. This was achieved by combining the analyses of $\nu_{\mu}$ and $\bar{\nu}_{\mu}$ disappearance and $\nu_{e}$ and $\bar{\nu}_{e}$ appearance for both beam and atmospheric neutrino samples using a three-flavour neutrino oscillation model. Figure 3.3 shows the confidence intervals for the final MINOS three-flavour analysis measuring $\Delta m_{32}^{2}$ and $\theta_{23}$. 


\subsection{MINOS+}

MINOS+ [80] is the continuation of the MINOS detectors with the NuMI beam configured to produce neutrinos at higher energies. Figure 3.4 shows the the neutrino energy spectra for $\nu_{\mu}$ charged-current events observed at the Near Detector

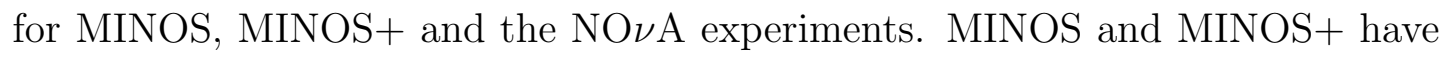
the Near and Far Detectors on-axis, that is, they are situated along the longitudinal direction of the neutrino beam. MINOS+ observes a high flux of neutrinos at higher energies compared to the low-energy configuration with the beam peak shifting from $3 \mathrm{GeV}$ to $7 \mathrm{GeV}$ due to a change in the neutrino beam configuration. The $\mathrm{NO} \nu \mathrm{A}$ experiment is off-axis, being placed $14 \mathrm{mrad}$ from the beam axis thereby increasing the number low-energy neutrinos around the oscillation dip, and heavily reducing the high-energy component.

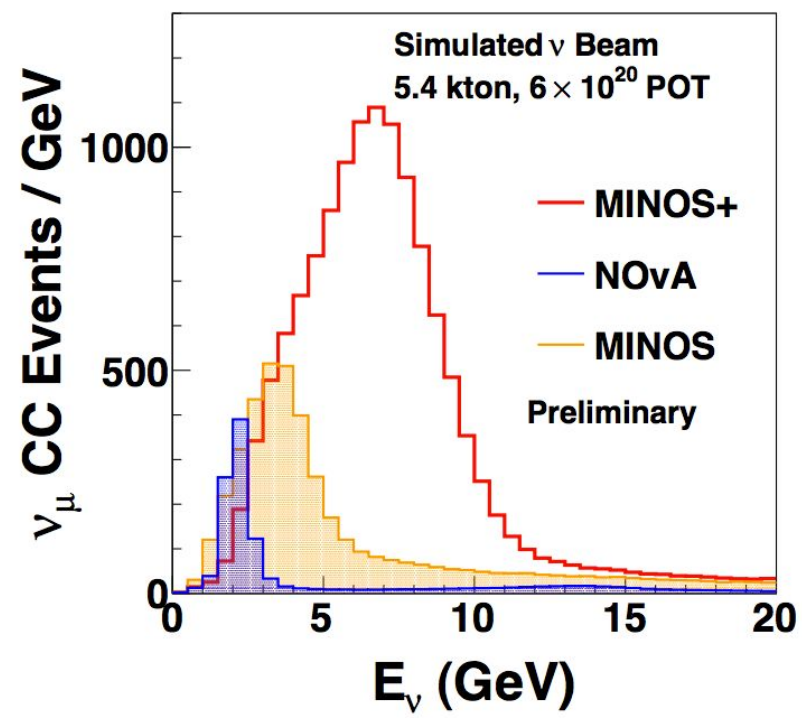

Figure 3.4: The neutrino energy spectra for $\nu_{\mu}$ charged-current events observed at the Near Detector for MINOS (yellow), MINOS+ (red) and the NO $\nu \mathrm{A}$ [52] (blue) experiments.

MINOS+ took data from September 2013 until June 2016, and with the additional statistics will provide important contributions to the high-precision tests of the three-flavour oscillation formalism and will improve on the world-leading measurements of muon-neutrino disappearance made by MINOS. The beam ex- 
posure as a function of time for MINOS+ can be seen in figure 3.2.

With more statistics at high energies MINOS+ is in the unique position to probe and significantly extend the reach of the search presented in this thesis for sterile neutrino signatures in the regions of parameter space favoured by experiments such as LSND and MiniBooNE. MINOS+ can also search for non-standard neutrino interactions [81] and other sources of new physics such as Large Extra Dimensions [82].

\subsection{The NuMI Beam}

The Neutrinos at the Main Injector (NuMI) neutrino beam [77, 83] was built at Fermilab to provide neutrinos for the MINOS experiment. The NuMI beam typically had a beam power of $350 \mathrm{~kW}$ with a design specification of up to $400 \mathrm{~kW}$. A high-intensity beam is required to achieve a meaningful event rate at the FD which is situated several hundred kilometres away. Such a distance significantly reduces the neutrino flux as it decreases as a function of distance away from the neutrino production target.

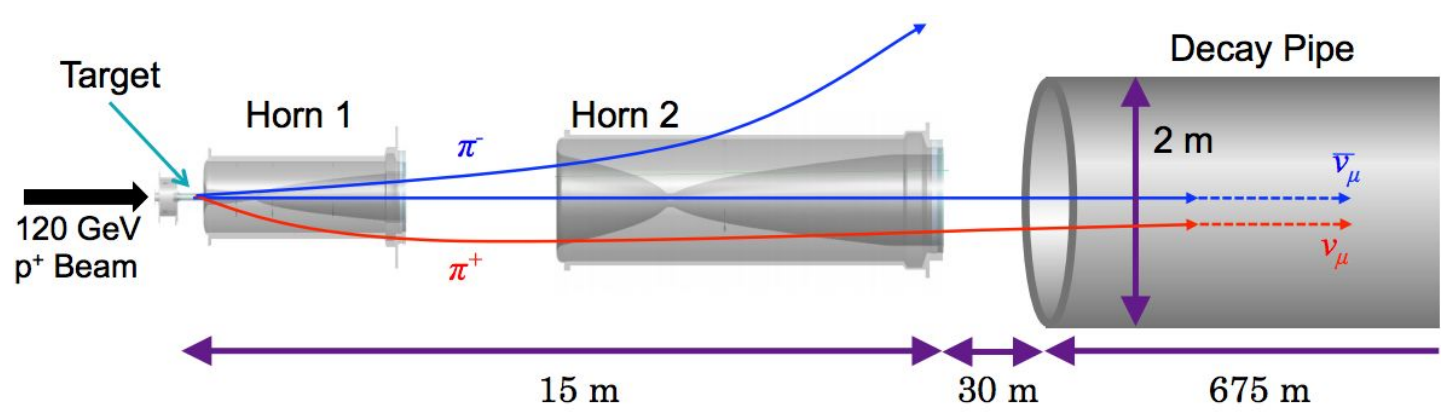

Figure 3.5: The NuMI beam.

To produce a neutrino beam the first stage is to create protons from $\mathrm{H}^{-}$ions. The ions are accelerated by a Radio Frequency (RF) quadrupole up to an energy of $750 \mathrm{keV}$. From there a linear accelerator accelerates the ions to an energy 
of $400 \mathrm{MeV}$, which subsequently pass through a thin carbon foil stripping the electrons off the ions to leave a beam of protons. The protons are fed into a rapid cycling synchrotron (Booster) and accelerated in batches up to energies of $8 \mathrm{GeV}$. Subsequently they are fed into the Main Injector where they are accelerated to $120 \mathrm{GeV}$. The Main Injector has a circumference seven times larger than the Booster and so up to six batches of protons can be inserted into the Main Injector at once with space to allow for the rise time of the pulse kicker. The pulse kicker bends the beam to align it towards the neutrino production target. It is the interaction between these high energy protons upon the fixed graphite target that results in a plethora of charged pions, kaons and muons.

Figure 3.5 shows how the charged particles pass through two parabolic, magnetic horns which focus either positive or negative particles depending on the direction of the electric current being pulsed through the horns into the decay pipe. The decay of muons into electrons contributes an intrinsic electron neutrino component in the beam. The focused hadrons travel along a $675 \mathrm{~m}$ decay pipe filled with helium. It is the decay of these particles inside the pipe that forms the predominately muon-flavour neutrino beam:

$$
\begin{aligned}
& \pi^{ \pm} \rightarrow \mu^{ \pm}+\nu_{\mu} \backslash \bar{\nu}_{\mu}, \\
& K^{ \pm} \rightarrow \mu^{ \pm}+\nu_{\mu} \backslash \bar{\nu}_{\mu}, \\
& \mu^{ \pm} \rightarrow e^{ \pm}+\bar{\nu}_{\mu} \backslash \nu_{\mu}+\nu_{e} \backslash \bar{\nu}_{e} .
\end{aligned}
$$

By focusing the positive hadrons a beam of predominately $\nu_{\mu}$ is created $\left(\nu_{\mu^{-}}\right.$ dominated beam mode); by focusing the negatively charged hadrons the $\bar{\nu}_{\mu}$ component can be increased $\left(\bar{\nu}_{\mu}\right.$-enhanced beam mode). The relative contribution of neutrino parents as a function of true neutrino energy for the low-energy neutrinodominated beam is shown in figure 3.6. Pions are the main contribution, with a significant kaon contribution at higher energies. Figure 3.7 shows three of the 
possible energy spectra observed at the Near Detector by varying the current sent to the focusing horns and the relative position of the neutrino production target to the horns.

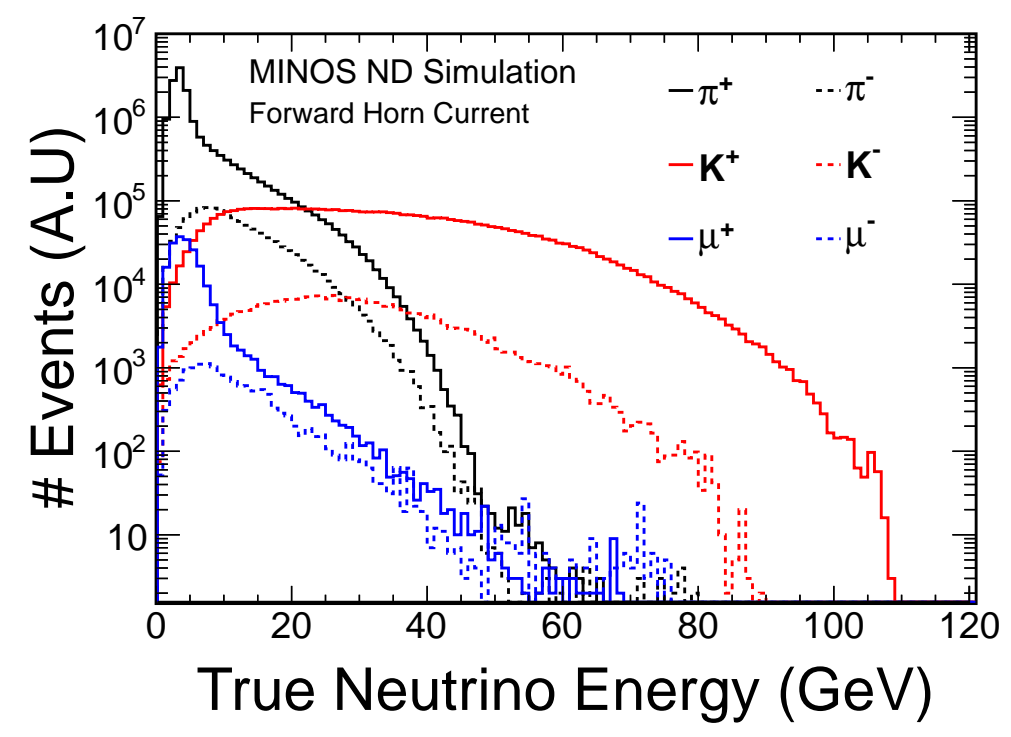

Figure 3.6: Simulated events at the MINOS ND for the neutrino-dominated lowenergy beam. The graph shows the relative contribution of the neutrino parents as a function of true neutrino energy.

The compositions of the NuMI neutrino-dominated and antineutrino-enhanced beams for charged-current neutrino interactions observed in the MINOS Near Detector are compared in figure 3.8. The significant difference in composition and event rate between these beam modes arises mainly from the fact that the $\bar{\nu}_{\mu}$ interaction cross section is a factor of approximately two lower than the $\nu_{\mu}$ interaction cross section.

\subsubsection{Slip-Stacking}

A method called "Slip-Stacking" can be performed in the Main Injector to allow the intensity of a proton batch to approximately double by merging two batches together in the Main Injector, therefore increasing the power of the NuMI beam [84]. The Main Injector cannot accommodate more than six batches 


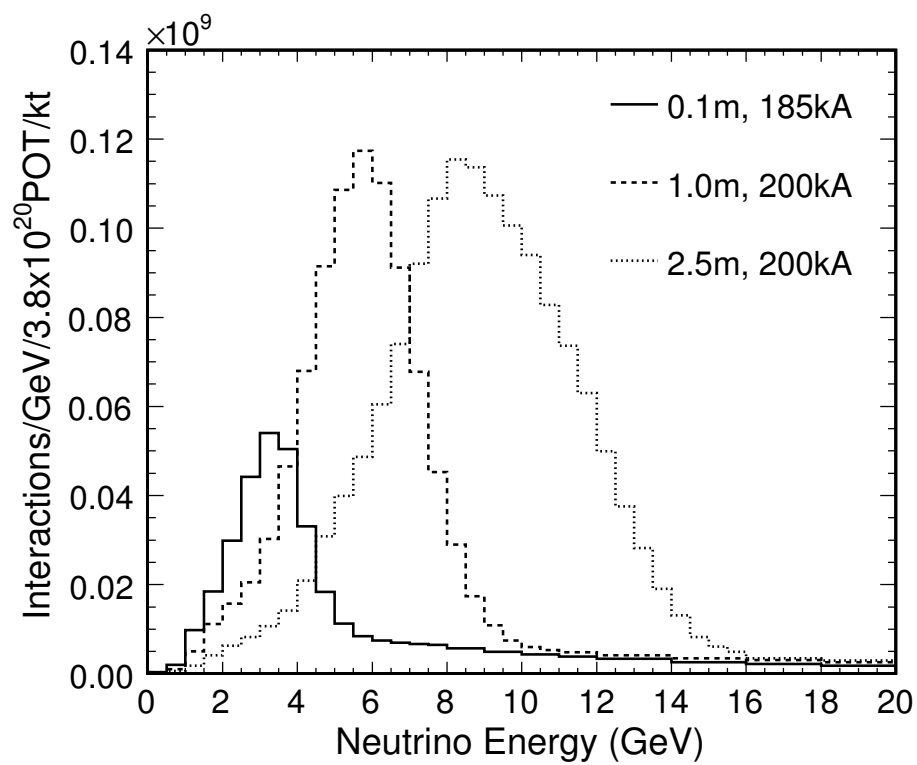

Figure 3.7: The true energy distribution of Near Detector events from the three different NuMI configurations. The target position (distance upstream of a nominal position) and horn current of each configuration are shown in the legend. " $0.1 \mathrm{~m}, 185 \mathrm{kA}$ " is the "low-energy" configuration in which most of the MINOS data has been taken. " $1.0 \mathrm{~m}, 200 \mathrm{kA}$ " is the "medium-energy" configuration which is the beam configuration in the $\mathrm{NO} \nu \mathrm{A}$ era and therefore is the beam setup in which MINOS+ took data. The final configuration is the "pseudo-high-energy" configuration.

at once, however by using three different RF cavities operating at different frequencies additional batches can be injected and coalesced together to produce double-intensity batches. The procedure to produce a slip-stacked batch is as follows:

1. A proton batch is injected from the Booster into the Main Injector at an energy of $8 \mathrm{GeV}$ and is captured by the first RF system.

2. The frequency of the RF system is lowered resulting in the deceleration of the batch, causing its momentum to decrease, and hence it will have a smaller orbit around the Main Injector circumference. A second proton batch is injected into the Main Injector and is captured by a second RF system operating at a higher frequency than the first (a larger momentum and therefore orbit). 

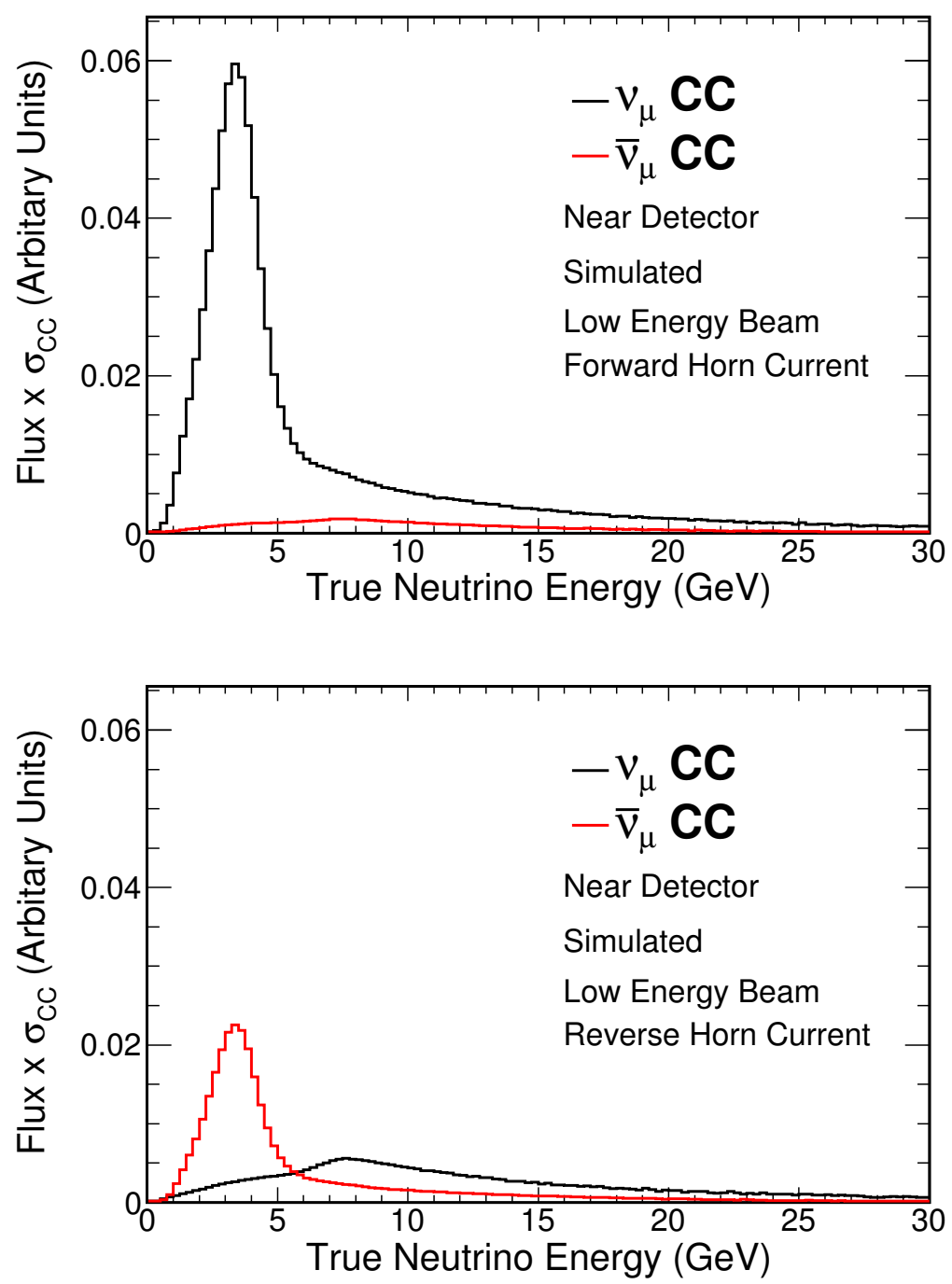

Figure 3.8: The reconstructed neutrino energy spectra at the MINOS Near Detector. The top graph shows the energy spectra produced when focusing positivelycharged hadrons producing a predominately $\nu_{\mu}$ beam. Below is when negatively charged hadrons are focused, increasing the number of $\bar{\nu}_{\mu}$ events seen at the detectors. Note how in anti-neutrino mode the event rate is significantly less due to different cross sections between neutrinos and anti-neutrinos.

3. The two batches are moving at different speeds relative to another around the Main Injector. The two batches continue to accelerate around until they are aligned longitudinally where they are both picked up by a third RF system with a frequency which is an average of the first and second RF system. As a result the two batches coalesce and can be treated as a single batch with double the intensity. 
Figure 3.9 shows the structure of a six-batch beam spill in which the first three batches have been slip-stacked.

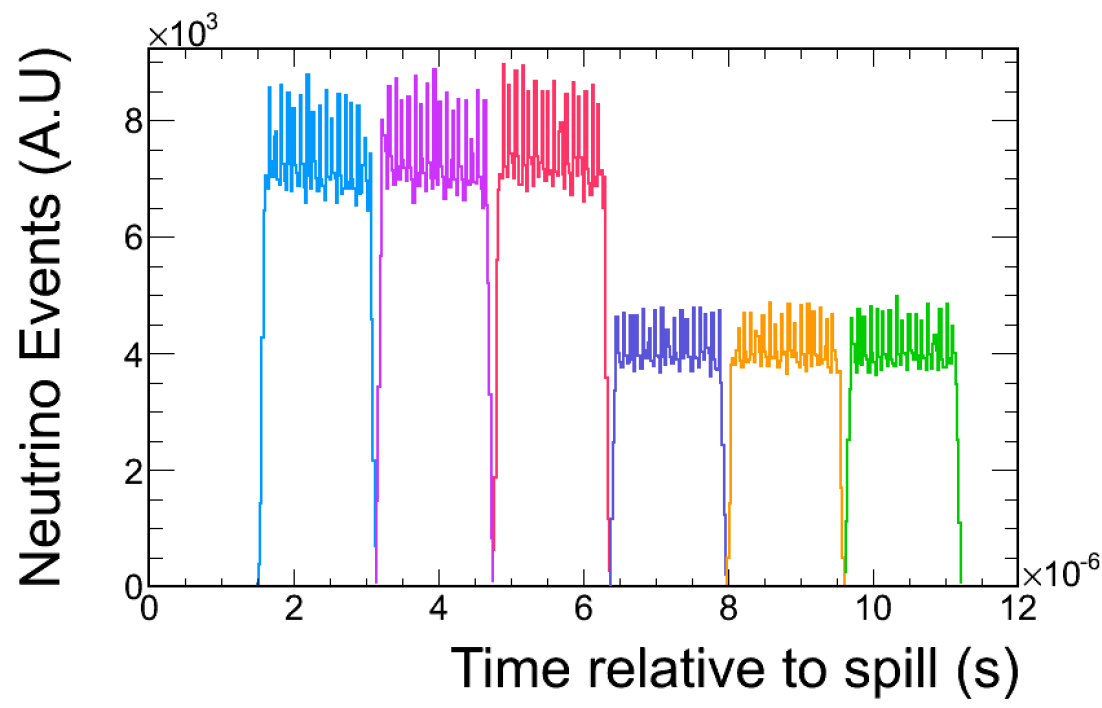

Figure 3.9: Reconstructed neutrino events at the Near Detector (data). The $x$ axis is the time of the reconstructed neutrino event relative to the trigger that indicates a NuMI beam spill. The different colours indicate the different proton batches. The data shows the beam was delivering six proton batches. Three of the batches have been doubled in intensity due to being slip-stacked.

\subsubsection{The NuMI Target}

The NuMI target, shown in figure 3.10, is composed of 47 graphite fins. Each fin is $6.4 \mathrm{~mm}$ wide, $15 \mathrm{~mm}$ tall and $20 \mathrm{~mm}$ long with a spacing of $0.3 \mathrm{~mm}$. The total thickness of all 47 fins is $954 \mathrm{~mm}$ which is approximately 2 hadronic interaction lengths. The thin-fin design optimises the proton-carbon cross section whilst minimising the number of secondary hadronic interactions from pions and kaons that would reduce the neutrino flux. Figure 3.10 also shows the fins are placed in an aluminium casing running parallel to two water lines for cooling.

The lifetime of a NuMI target is finite. During the duration of the MINOS-era data-taking seven NuMI targets were used. Assuming the target is not subjected to any serious issues such as water leaks, or physical damage through an accident, the graphite will begin to degrade due to the periodic stresses, impacts and radi- 


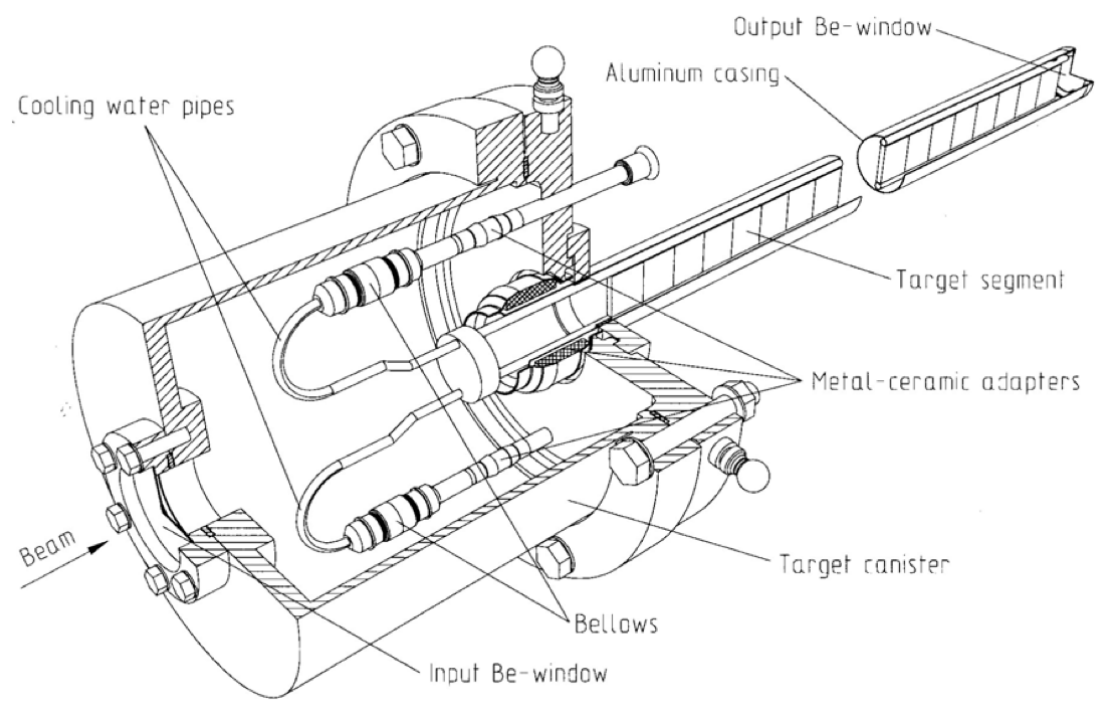

Figure 3.10: Technical drawing of NuMI target and target housing used in the MINOS experiment. Image taken from [85].

ation from the beam. As a result the target will break up and become less dense and the neutrino flux will decrease. Chapter 9 will present the effect target decay has had on the neutrino energy spectrum for the dataset used in this thesis.

\subsection{The MINOS Detectors}

\subsubsection{Overview}

The MINOS detectors are tracking, sampling calorimeters made of alternating layers of $1 \mathrm{~cm}$ thick plastic scintillator and $2.54 \mathrm{~cm}$ thick steel planes; figure 3.11 shows how the plastic and steel are used to form a plane. Each adjacent plane is rotated by $90^{\circ}$ with respect to its neighbour to allow for $3 \mathrm{D}$ reconstruction in the detectors. MINOS identifies events by observing the light produced by the final state charged particles in the scintillator. The scintillation light is sent along wavelength-shifting fibre and read out by Photomultiplier tubes (PMTs). Both detectors are magnetised so that the charge of a muon interacting within the detector can be inferred by the direction of bending. A co-ordinate system is defined as $x$ being the horizontal direction perpendicular to the beam, $y$ the 
vertical direction, and the beam direction defined as $z$. An alternative righthanded co-ordinate system is also defined with the beam direction still defined as $z$ but a $U V$ plane being defined as a $45^{\circ}$ rotation to the $x y$ plane as displayed in figure 3.11. Both detectors are explained in great detail in [76].

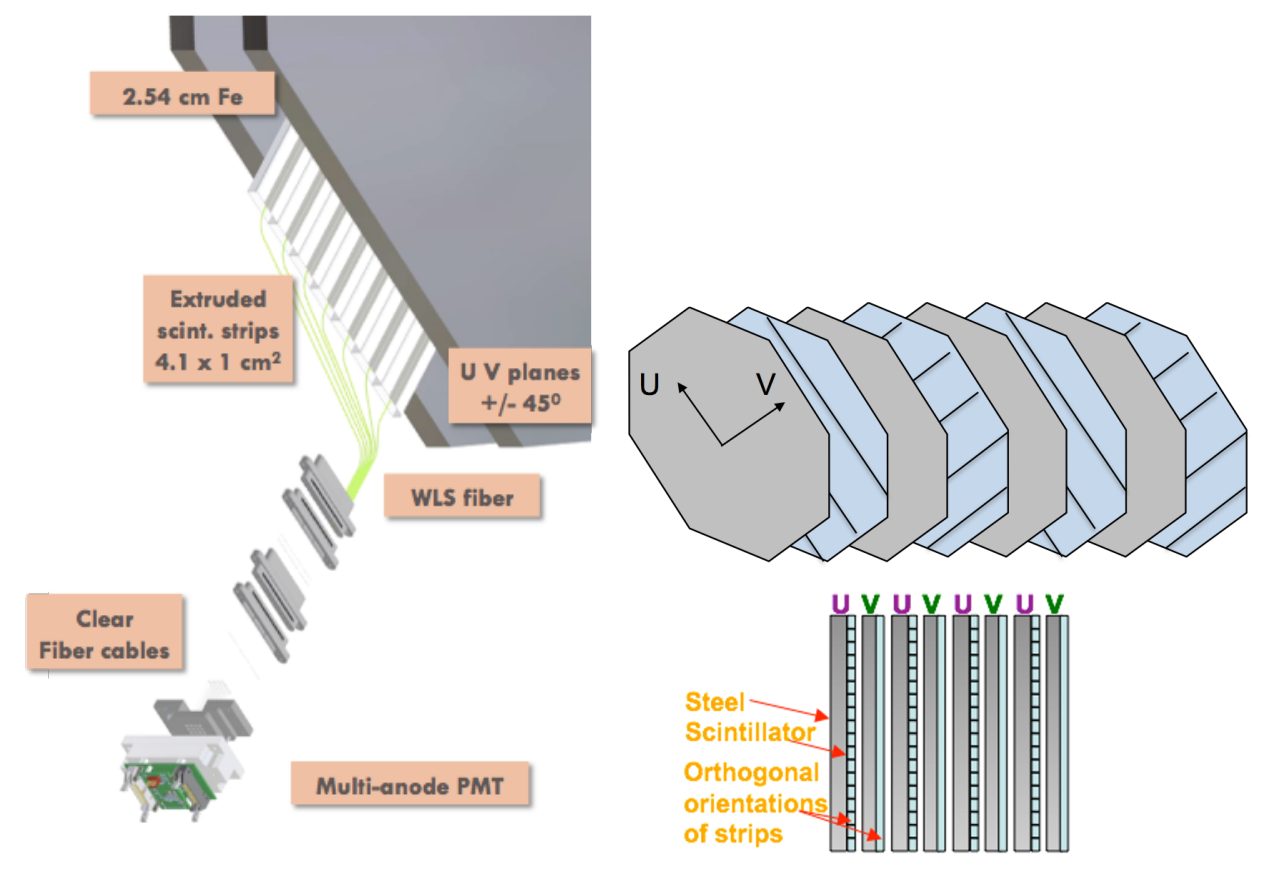

Figure 3.11: The components of a MINOS plane comprise plastic scintillator and a steel plane (left). The arrangement of multiple planes at $90^{\circ}$ to one another labelled $U$ and $V$ to allow for 3D reconstruction in the detectors is shown on the right.

\subsubsection{Two-Detector Experimental Setup}

MINOS is composed of two detectors, the ND and the FD. Both detectors are designed to be similar from the materials used in construction to their functionality. By looking at the relative differences between the reconstructed neutrino energy spectra in the two detectors rather than measuring the absolute neutrino flux, the impact of experimental systematic errors that affect both detectors are significantly reduced. Any mis-modelings such as neutrino cross-sections and flux will affect both detectors in the same way and therefore a relative comparison will cancel out any such effects. The analysis in this thesis will take advantage of 
this by fitting the ratio of the FD to ND energy spectra.

\section{Near Detector}

The ND lies 110 m underground, which corresponds to 225 meters-water-equivalent (mwe) overburden, and is $1.04 \mathrm{~km}$ downstream from the NuMI production target. The beam is powerful enough at this point to produce many tens of neutrino interactions per proton spill from the NuMI beam. Such a large flux allows the ND to be the smallest of the two detectors with a mass of 980 tonnes. The ND can be seen in figure 3.12 ; the detector is $4.8 \mathrm{~m}$ wide and $3.8 \mathrm{~m}$ tall with the coil hole for producing the magnetic field being off centre. The beam axis is displaced $1.48 \mathrm{~m}$ from the coil hole to reduce the number of particles depositing energy within the coil hole which is not well modelled in simulation.

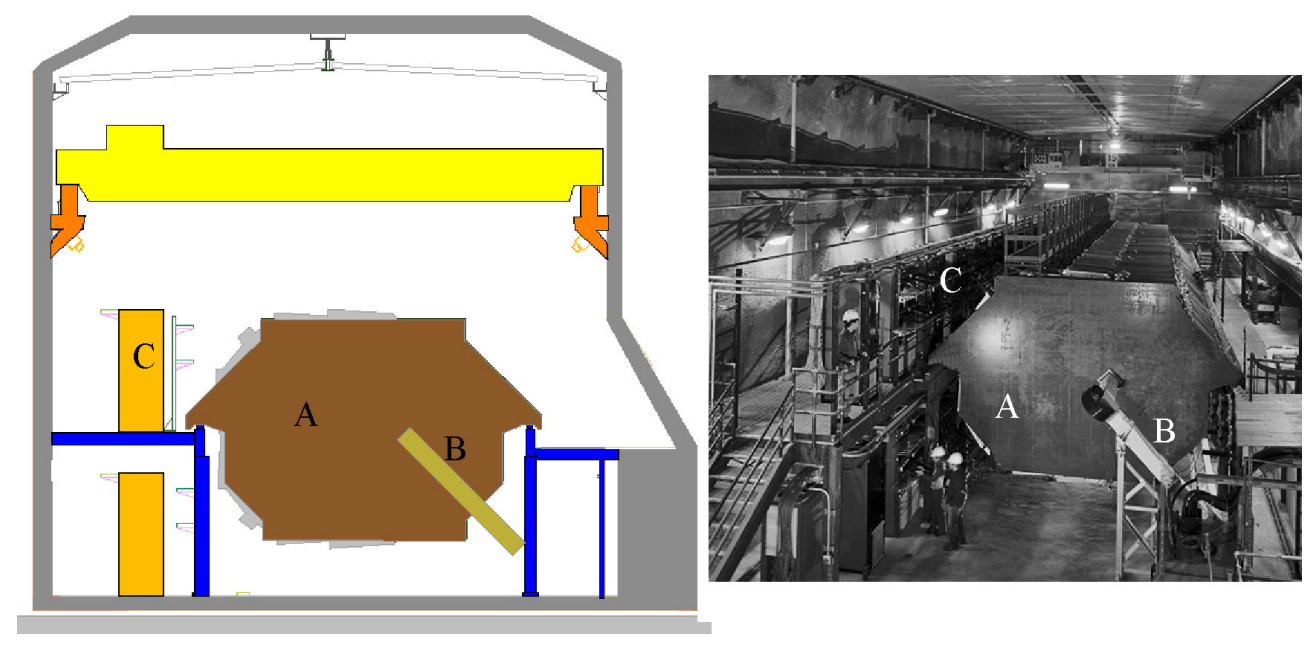

Figure 3.12: End view of the MINOS ND (the NuMI beam going into the page). The drawing (left) helps illustrate and label the image of the MINOS ND (right). "A" is furthest upstream steel plane, "B" is the magnet coil, and "C" is the electronics rack. The NuMI beam axis is aligned near the "A" label. Image taken from [76].

The ND consists of 282 steel planes. The upstream part of the detector (the first 120 planes) is the calorimeter section and all except the first plane are instrumented with scintillator. Within the calorimeter section one in every five planes is fully instrumented, the others having a smaller region of scintillator around the 
beam axis. The calorimeter is deigned to measure hadronic energy depositions. The remaining 162 planes act as the spectrometer, used to measure the momenta of energetic muons. Every fifth plane is instrumented with scintillator whilst the others have none.

\section{Far Detector}

The FD is $735.3 \mathrm{~km}$ downstream from the NuMI production target and $705 \mathrm{~m}$ underground (2070 mwe). The FD, shown in figure 3.13, consists of 484 octagonal steel planes constructed as two super-modules separated longitudinally by $1.15 \mathrm{~m}$. To compensate for the reduced flux from the neutrino beam (a factor of $\sim 10^{5}$ ) the FD is much larger than the ND with a mass of 5400 tonnes. The detector sees on average three neutrino interaction events per day in the low-energy NuMI beam.

The FD super-modules each have their own independently operated magnet coil. The super-module closest to the NuMI beam has 249 planes and is $14.78 \mathrm{~m}$ in length. The second super-module has 237 planes and is $14.10 \mathrm{~m}$ in length. No scintillator is instrumented onto the first plane of each super module.

A veto shield located around the top of the FD (over both super-modules) enables the selection of atmospheric neutrino events from the data [86]. The atmospheric neutrinos are selected from a large background of cosmic muons. The 2070 mwe overburden suppresses a significant amount of the comic ray background, however high energy cosmic muons can travel through the earth's crust and enter the FD from above. Cosmic muons will have to pass through the veto shield which is made of the same scintillator modules as the scintillator planes in the detectors.

There are a number of reasons why a cosmic muon may not be tagged by the veto shield: a shield readout channel is dead due to noise or damage from radioactivity, a cosmic muon passes through the gap between the two modules or 

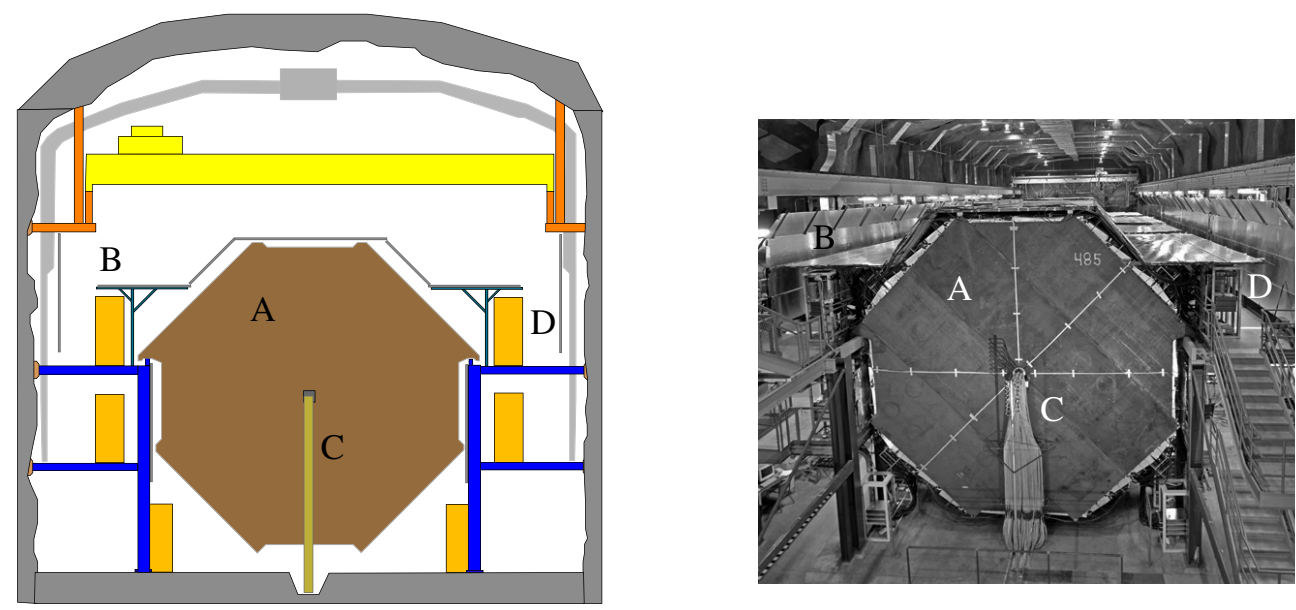

Figure 3.13: The end view of the MINOS FD (beam coming out of page). The drawing (left) helps illustrate and label the image of the MINOS FD (right). "A" is the most downstream steel plane, " $\mathrm{B}$ " is the cosmic ray veto shield, " $\mathrm{C}$ " is the end of the magnet coil, and " $\mathrm{D}$ " is the electronics rack. Image taken from [76].

the activity left by the cosmic muon in the veto shield was too small to register a signal.

\subsection{Steel}

The steel has an average density of $7.85 \pm 0.03 \mathrm{~g} \mathrm{~cm}^{-3}[76]$. The high density increases the chance of a neutrino interaction within the detector. The typical RMS deviation of plane masses is $0.35 \%$ (the plane thickness varying by $0.3 \%$ ). Neighbouring steel planes are $5.95 \mathrm{~cm}$ apart [76].

\subsection{Plastic Scintillator}

As neutrinos travel through the detector they interact with the iron nuclei, and the charged final-state particles travel through the scintillator where the energy deposition is read out as light. The structure of a strip of scintillator can be seen in figure 3.14. The plastic-scintillator strips are made from extruded polystyrene, doped with organic scintillators PPO (1\% by weight) and POPOP $(0.03 \%$ by 
weight). The strips are coated with a reflective, uniformly distributed, thin $(0.25 \mathrm{~mm})$ layer of $\mathrm{TiO}_{2}$ (15\% by weight) that serves as a diffuse reflector to improve light yield [76]. The scintillator strips were produced by Itasca Plastic, producing over 300 tons of scintillator with the light emission spectrum peaked at $\sim 420 \mathrm{~nm}$. Each strip has a groove along the surface containing a $1.20_{-0.01}^{+0.02} \mathrm{~mm}$ diameter wavelength-shifting (WLS) fibre.

The WLS fibres are produced by Kuraray, Inc. in Japan and consists of double-clad polystyrene fibre with 175 ppm of Y11 (K27) fluorescent emitter. The WLS fibres minimise self-absorption by absorbing light peaked at $\sim 430 \mathrm{~nm}$ and re-emitting it at a peak of $470 \mathrm{~nm}$ and beyond (from blue to green). The wavelength-shifted light is transported to an optical connector at the edge of the scintillator and into clear polystyrene fibres that transport the light to PMTs. More detailed accounts of the cost-effectiveness and practicality behind using a plastic-scintillator in the MINOS experiment can be found at [87].

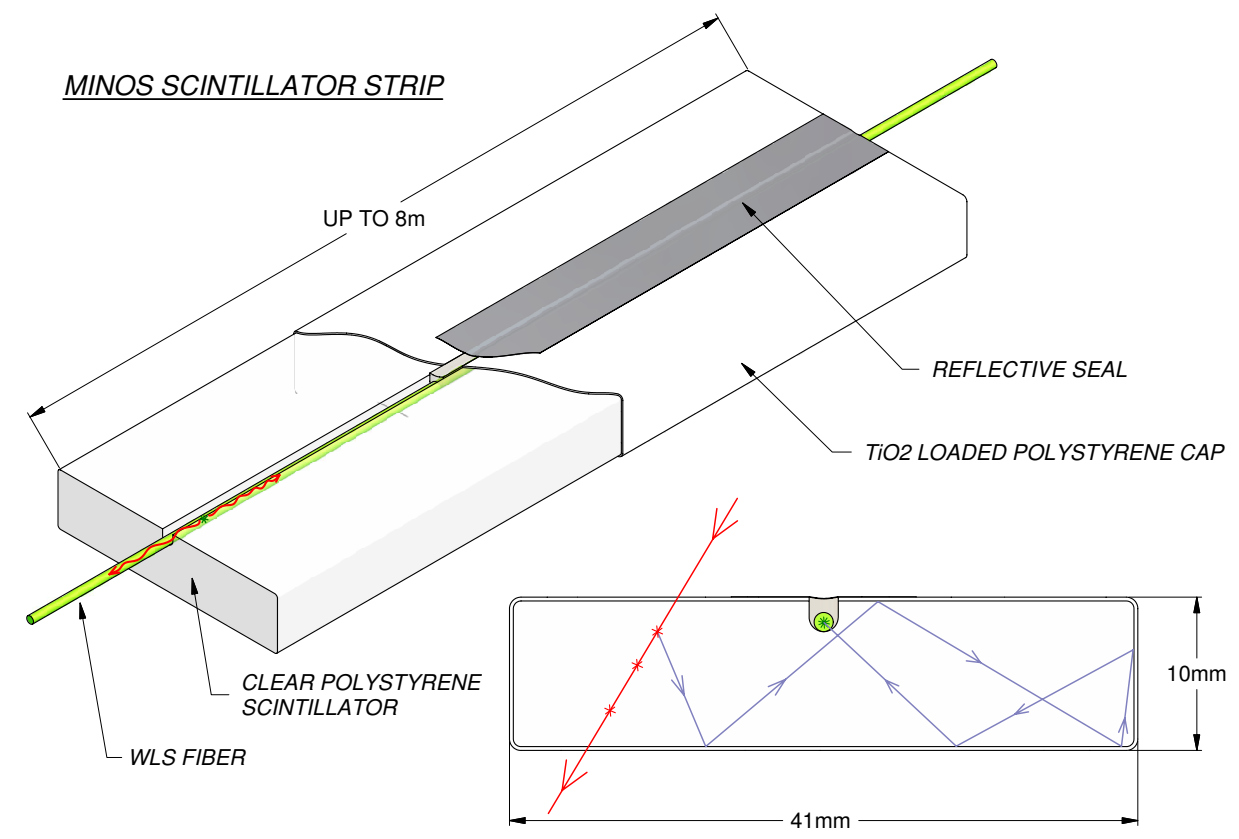

Figure 3.14: A strip of MINOS scintillator with the WLS fibre installed along the centre. The image in the lower right corner depicts a charged particle (red line) depositing energy by producing light (blue line) as it passes through the strip. The light travels along the WLS fibre and is read out by a PMT. Image taken from $[76]$. 
The scintillator strips are closely packed into modules of 16 and 28 strips that are wrapped in aluminium to ensure a light-tight enclosure. The module design is advantageous as it provides a mechanically strong structure that holds strips together and is sufficiently robust for shipping and mounting to the steel plates. Once these modules are attached to the steel plates a MINOS steel-scintillator plane is complete. The designs for the Near and Far Detectors differ slightly and can be seen in figures 3.15 and 3.16 .
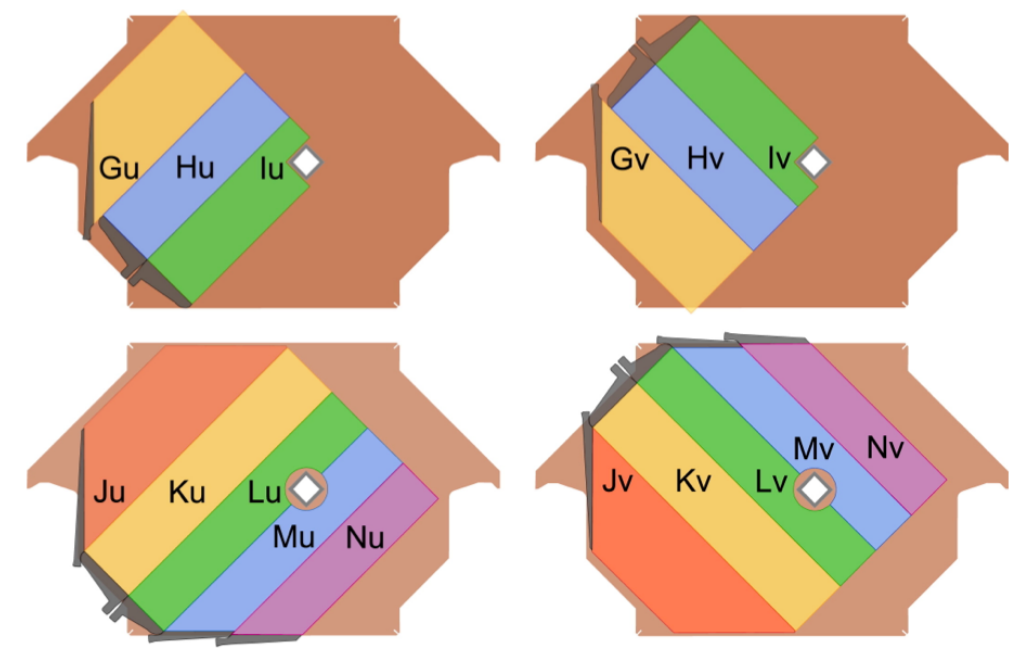

Figure 3.15: The four different configurations of planes used in the ND, showing the different layouts of the scintillator modules. The figure shows partially instrumented $U$ planes module (top left), partially instrumented $V$ (top right), fully instrumented $U$ (bottom left) and fully instrumented $V$ (bottom right) where $U$ and $V$ are the coordinates used. Figure taken from [76].

\subsection{Photomultiplier Tubes}

The scintillation light is transported by the WLS fibres to clear optical wire bundles that transport the light to Hamamatsu multi-anode photomultiplier tube pixels. At the FD, 16-anode (Hamamatsu R5900-00-M16) PMTs are used with gains up to $1 \times 10^{6}$. The ND uses 64-anode (Hamamatsu R5900-00-M64) PMTs with gains of $0.8 \times 10^{6}$. The voltage required to achieve such gains for these PMTs is $\sim 800 \mathrm{~V}$. The PMTs are encased in boxes to ensure they are light-tight and to 


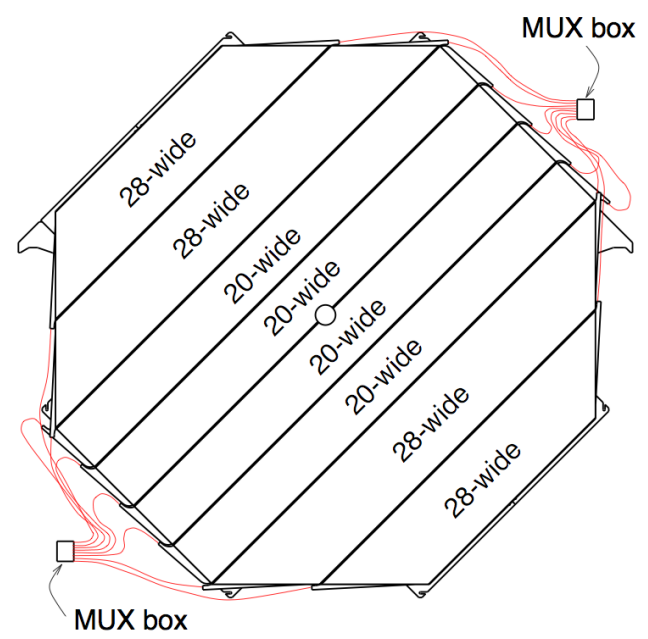

Figure 3.16: The arrangement of the scintillator modules on a MINOS Far Detector plane. Note that every plane at the Far Detector is fully instrumented. Figure taken from [76].

shield them from magnetic fields and ambient electronic noise [76].

At the FD, each scintillator strip is read out at both ends requiring 1452 PMTs. Eight scintillator strips are read out by one PMT pixel; the WLS fibres are "multiplexed" onto the PMT pixels, meaning eight fibres are connected to a single pixel, with the fibre-to-pixel mapping intentionally different at each end of each strip allowing for the determination of correct track location with an eight-fold reduction in pixels. This setup yields a light output similar to the ND scintillator strips which are read out at one end and mirrored at the other; this is achievable due to to the shorter length of the ND scintillator strips. The ND uses 194 PMTs.

\subsection{Magnetic Field}

Both the FD and ND are magnetised by a current-carrying coil. The geometric differences between the FD and ND mean different requirements for coils. Both detectors have a magnet coil passing through a hole along the whole length of the detector, equipped with water cooling. The FD has an independent magnet coil [88] within each super-module. At the FD the average toroidal magnetic field 
is $1.42 \mathrm{~T}$ whereas the ND has an average toroidal magnetic field of $1.28 \mathrm{~T}$ [89].

Magnetised detectors allow the MINOS experiment to distinguish oppositely charged muons on an event-by-event basis. This allows MINOS to distinguish $\nu_{\mu}$ and $\bar{\nu}_{\mu}$ charged-current interactions within the detectors, allowing the collaboration to measure the oscillation parameters of anti-neutrinos and neutrinos separately providing a test of CPT violation [90, 91].

\subsection{Electronics and Readout}

The electronics differ between the detectors due to the difference in the number of neutrino interactions each detector experiences. The FD observes fewer neutrino interactions by many orders of magnitude due to the long-baseline from the NuMI target and the large overburden to suppress cosmic rays. The rate of cosmic ray interactions in the FD is $0.5 \mathrm{~Hz}$ with the majority of the data volume dominated by detector noise [92]. The FD electronics are explained in detail in [93].

The ND electronics are designed to address the larger instantaneous event rate due to the shorter distance from the production target. The NuMI beam is designed to deliver a high-intensity neutrino beam, which can produce an order of 10 neutrino events per beam spill in the ND. In order to accurately reconstruct each event the electronics are required to be fast and have no dead time during the $10 \mu$ s duration of a beam spill. More details on the ND electronics can be found in [94].

\subsection{Light Injection}

The MINOS light injection system monitors the stability and linearity of the PMTs used in the calibration chain discussed in section 4.1. Each detector is equipped with a light injection system that allows varying intensity pulses of ultra-violet light produced by LEDs (light-emitting diodes) to be injected into the 
FD and ND. The light injected into the detectors from the LEDs is monitored by PIN (Positive Intrinsic Negative) photodiodes, the signal from which is reshaped to mimic the signal from scintillation light read out by the PMTs. The signal from the photodiodes is then read out by the standard electronics of the detectors.

\subsection{Triggering}

The NuMI beam delivers the neutrinos in "spills". When a beam spill occurs, a signal (spill trigger) is sent to the ND and FD triggering a detector readout window for $100 \mu \mathrm{s}$ around each spill. The FD requires additional triggers to address the high level of noise originating from dark noise from within the PMTs and spontaneous light emission in the WLS fibres [92]. A trigger is implemented that requires 2 out of 36 PMTs to produce a signal readout within a $400 \mathrm{~ns}$ window. Each set of 36 PMTs is read out and once the trigger is activated the data is digitised. Outside of the beam spill window offline triggers cause data from cosmic muons to be recorded and stored, basing the decision on either a minimum amount of energy deposited in a set of planes, or a certain number of planes hit within a group of planes.

\subsection{The Calibration Detector}

The Calibration Detector (CalDet) is the smallest of the MINOS detectors. CalDet was designed and built to measure the calorimetric response to hadrons and electrons, and the topology of hadronic and electromagnetic interactions in a number of test beams at CERN [95]. CalDet was built to be of similar design to the MINOS ND and FD to demonstrate they can be calibrated relative to one another to high precision. CalDet consisted of 60 unmagnetised planes, each plane a $1 \mathrm{~m}$ square of the same steel and scintillator composition as the two main MINOS detectors. However, the steel planes were $\sim 0.04 \mathrm{~cm}$ thinner in the CalDet detec- 
tor corresponding to a decrease in thickness of $1.6 \%$ meaning the average energy deposition of a particle of known energy will be $\sim 1.6 \%$ higher in CalDet relative to the ND and FD. Each plane contained 24 scintillator strips, each $100 \mathrm{~cm}$ long. In an identical arrangement to the ND and FD, the planes were aligned so that adjacent planes were at $90^{\circ}$ to each other. Both the ND and FD electronics were implemented at CalDet during testing to understand and quantify any differences in the read-out electronics; the responses were found to agree to within $0.06 \%[96]$.

The CalDet detector, through subjection of several test-beams of well-defined particle energies, was used to develop a calibration chain (discussed in section 4.1) to allow the ND and FD responses to particle interactions to be related to one another, acheiving an uncertainty in the relative calibration between the ND and FD of $\sim 2 \%[95]$.

\subsection{Neutrino Interactions in the Detectors}

\subsubsection{Event Topologies}

There are three neutrino interactions that are of interest to MINOS as shown in figure 3.17. The main channel is the charged current (CC) $\nu_{\mu}\left(\bar{\nu}_{\mu}\right)$ interaction,

$$
\nu_{\mu}\left(\bar{\nu}_{\mu}\right)+X \rightarrow \mu^{-(+)}+X^{\prime}
$$

The cascade of hadrons, $X^{\prime}$, produces a diffuse shower of energy deposits near the interaction vertex and a long muon track. MINOS was constructed with steel planes so that it can contain a significant proportion of the final state muons. The muon curves due to the magnetic field. It is the direction of curvature that allows MINOS to identify the incoming neutrino as a $\nu_{\mu}$ or a $\bar{\nu}_{\mu}$.

All active neutrino flavours undergo the neutral current (NC) interaction 

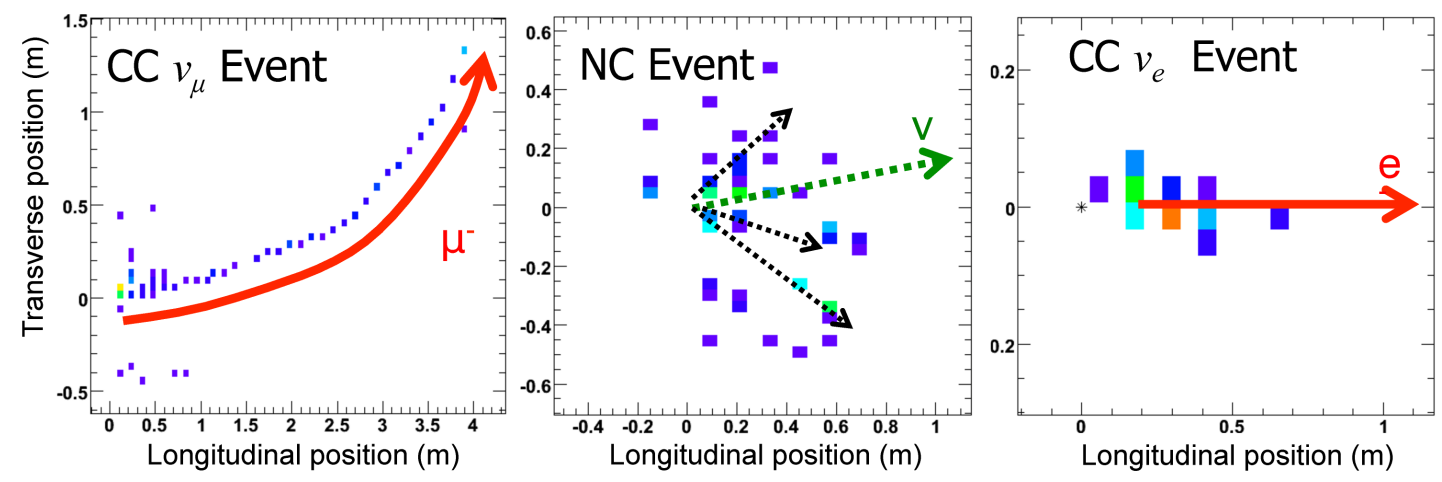

Figure 3.17: Neutrino interaction topologies observed in the MINOS detectors. Left: A charged current $\nu_{\mu}$ interaction. Middle: A neutral current interaction. Right: A charged current $\nu_{e}$ interaction. Each coloured pixel represents a scintillator strip with energy deposited from a charged particle. The colour scale displays the amount of light: purple and blue are low light levels, through to orange and red for the highest light levels.

through the process

$$
\nu+X \rightarrow \nu+X^{\prime}
$$

Only the hadronic shower is observed, producing a diffuse pattern of energy deposits within the detector. It is not possible to determine the flavour of neutrino.

Finally, electron neutrinos undergo $\mathrm{CC}$ interactions through the process

$$
\nu_{e}\left(\bar{\nu}_{e}\right)+X \rightarrow e^{-(+)}+X^{\prime}
$$

The electron gives rise to an electromagnetic shower, which produces a much denser, more compact shower of energy deposits. This interaction is difficult to identify due to the steel plate thickness, meaning that it can be difficult to separate these dense electromagnetic showers from the more diffuse hadronic showers.

\subsubsection{Neutrino Cross Sections}

The neutrinos used in the analysis presented in this thesis have energies in the range of $0.25-40 \mathrm{GeV}$. Within this energy range $\mathrm{CC}$ interactions can be divided into three dominant classes. 
1. Quasi-elastic scattering dominates below $1 \mathrm{GeV}$ :

$$
\begin{aligned}
& \nu_{\mu}+n \rightarrow \mu^{-}+p, \\
& \bar{\nu}_{\mu}+p \rightarrow \mu^{+}+n .
\end{aligned}
$$

At this energy neutrinos can elastically scatter off an entire nucleon liberating a nucleon (or multiple nucleons) from the target. In MINOS, quasielastic interactions result in little or no hadronic activity leaving only a muon track visible in the detector.

2. At a few $\mathrm{GeV}$, resonance production becomes important. In this process, the struck nucleus $(N)$ is excited to a baryon resonance $\left(N^{*}\right)$ that most likely decays to a nucleon-pion final state:

$$
\begin{gathered}
\nu_{\mu}+N \rightarrow \mu^{-}+N^{*}, \\
N^{*} \rightarrow \pi+N^{\prime} .
\end{gathered}
$$

The pion can give a shower-like topology if it undergoes hadronic interactions, or else a track-like signature in the case of the $\pi^{ \pm}$.

3. Deep inelastic scattering (DIS) dominates above a few GeV. In this process, the neutrino has sufficient energy that it can resolve the individual quark constituents of the nucleon which manifests in the creation of a large hadronic shower.

Figure 3.18 shows the three dominating cross section processes in the energy range $0-100 \mathrm{GeV}$ for both neutrinos and anti-neutrinos. At MINOS the oscillation dip observed in muon-neutrino disappearance at the FD occurs just below $2 \mathrm{GeV}$; therefore CCQE neutrino interactions provide a large source of signal events for a neutrino oscillation analysis. A detailed review on the current state of neutrino 
cross sections can be found in [97]. Recently a new cross-section channel has been found around the quasi-elastic and DIS region called "multi nucleon correlation" [98]. This new channel is not included in the simulation software used in this thesis. Chapter 6 discusses the systematic uncertainties included in this analysis and due to the two detector setup at MINOS the cross section systematic uncertainty is shown to not be one of the dominant systematics. Therefore the impact of not modelling this additional channel in this analysis is considered negligible.
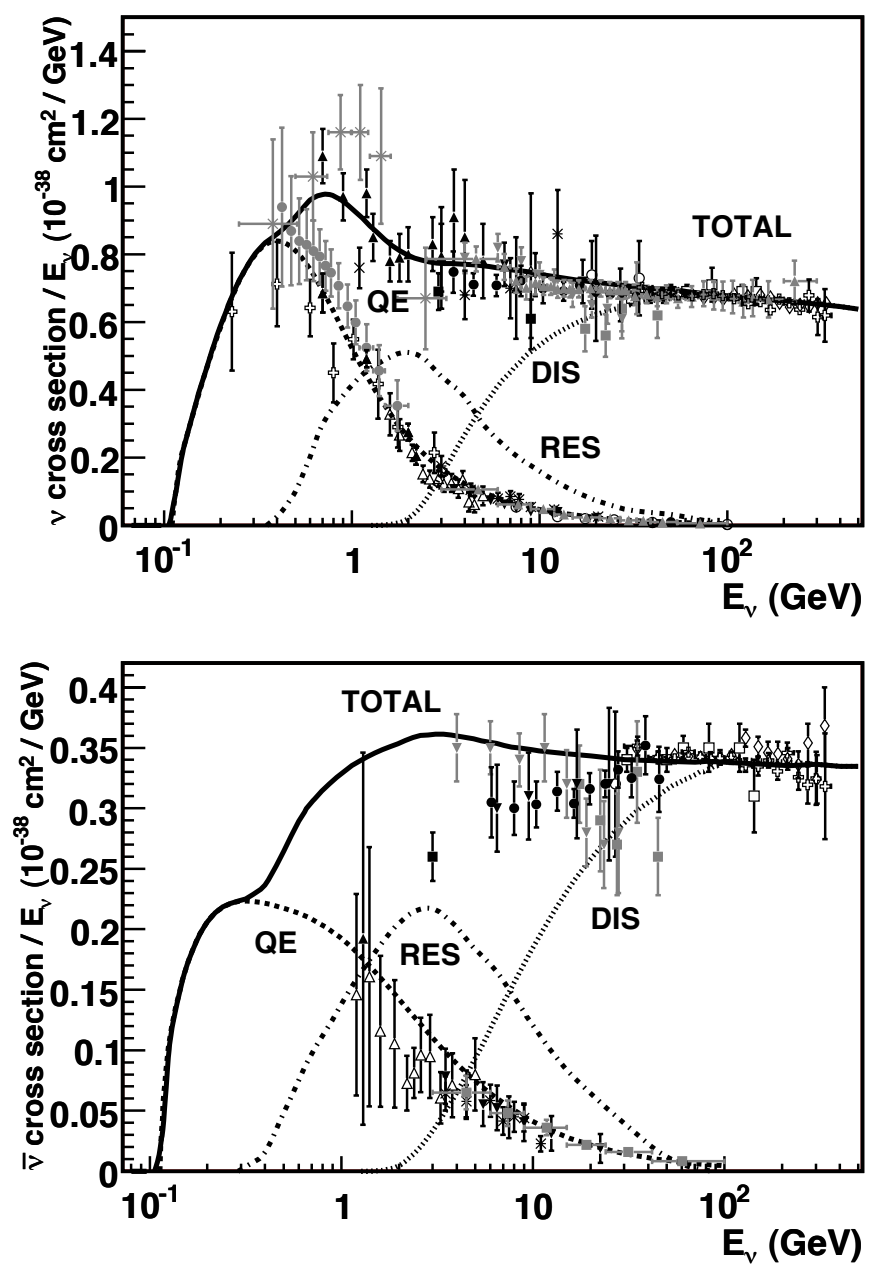

Figure 3.18: Total muon-flavoured neutrino (top) and anti-neutrino (bottom) charged-current cross-sections per nucleon. Figure taken from [97] 


\section{Chapter 4}

\section{Calibration and Reconstruction}

This chapter gives an overview of the calibration chain used in the MINOS detectors and a summary of the simulation packages used to generate simulated neutrino events to compare to data. The last section describes how the reconstruction software constructs neutrino events from track and shower energy depositions left in the MINOS detectors and the use of a multivariate algorithm to improve the shower energy estimation for charged-current events.

\subsection{Calibration}

The MINOS detectors are sampling calorimeters. Using the output of light produced by scintillation due to energy deposition from charged particles travelling through the detectors MINOS looks at the relative changes in the energy spectra and event characteristics between the two detectors to measure the neutrino oscillation parameters. It is by looking at the relative difference between the neutrino energy spectra at the ND and the FD that the experiment can perform precise measurements of the neutrino oscillation parameters. Therefore, the calorimetric energy scale must be well known; ideally this would be identical for both detectors. This chapter will cover a multi-stage calibration chain $[76,99]$ in which raw detector pulse height $Q_{\text {raw }}(s, x, t, d)$ read out from a strip, $s$, at a position along 
the strip, $x$, at a time, $t$, and in a detector, $d$, are converted to a fully-corrected signal $Q_{\text {corr }}(s, x, t, d)$ by applying several multiplicative calibration constants:

$$
Q_{\text {corr }}=Q_{\text {raw }} \times D(d, t) \times L\left(s, d, Q_{\text {raw }}\right) \times S(s, t, d) \times A(s, x, d) \times M(d),
$$

where $D(d, t)$ is a drift correction, $L\left(s, d, Q_{\text {raw }}\right)$ is a linearity correction, $S(s, t, d)$ a strip-to-strip calibration, $A(s, x, d)$ an attenuation of light correction and $M(d)$ is an energy scale factor. The terms are described individually below.

\subsubsection{Linearity Calibration}

A large energy deposition within a detector could result in a large number of photons from the scintillator. The linearity calibration addresses the non-linear response from the PMTs once light levels exceed $\sim 100$ photoelectrons $[100,101]$. On a monthly basis the LI system is used to deliver pulses of light of varying intensity, ranging from tens to hundreds of photoelectrons. By comparing the pulse height from the detector to that of the known signal injected via the LI system a pulse height dependent correction factor is calculated on a strip-bystrip basis, $L\left(s, d, Q_{\text {raw }}\right)$. The PIN diodes are an independent measure of the light output of the LEDs. They are known to be linear up to 1\% [100, 101] to signal sizes corresponding to that of the $\sim 100$ photoelectrons administered to the PMTs; above this threshold the non-linearity uncertainty doubles to $\sim 2 \%[100,101]$.

The ND electronics are corrected automatically using data obtained from special current injection runs [94]. The FD electronics has no such procedure and so an additional step in the calibration needs to be applied. Charge injection (CI) runs using a dedicated system in the FD front end electronics, it is similar to the light injection but bypasses the PMTs and allows for the monitoring of the linearity of the front end electronics. The LI system addresses the linearity 
of the PMTs and the electronics connected to them.

\subsubsection{Gain and Drift Calibration}

Gain and drift calibration, $D(d, t)$, accounts for the drift in the gains of the PMTs, the electronics response and the scintillator light output as a function of time.

Short term temperature fluctuations can cause variations in the electronics but are mostly eliminated by climate control of the detector caverns. However, seasonal climate changes combined with ageing of the electronics produces a $4 \%$ [76] reduction in overall detector response per year. The PMTs used at the ND and FD are multi anode PMTs controlled by a single voltage. With multiple channels per PMT it is not possible to obtain a fixed gain for each pixel accurately, therefore any differences must be calibrated out. The typical spreads in pixel gains of a 16-anode PMT are in the range of $15-25 \%$ [100]. The drift from the electronics is quantified by using the Light Injection (LI) system [102]. Periodically the LI system pulses light into the WLS fibres at both the ND and FD with each PMT pixel receiving $\sim 50$ photoelectrons per pulse [76]. Each month this average response per photoelectron of known energy can then be converted into a gain and used to calibrate out changes in the electronics at both detectors.

Although the LI system measures the time variations of PMT and electronics responses, the response of scintillators and WLS fibres cannot be measured in this way. The response of scintillators and WLS fibres varies as a function of time and temperature; typically one would expect $2-10$ [99] photoelectrons to be collected at the end of a scintillator strip from a minimally ionising muon passing perpendicular to the centre of the scintillator. The scintillator drift calibration is performed using though-going cosmic muons. Cosmic ray muons are treated as "standard candles" in MINOS and are used to track the response of each detector. Both the ND and FD experience a different rate and average energy from cosmic muons due to the relative difference in mass, rock overburden and 
geographical locations (the earth's magnetic field is not constant as a function of latitude) between both detectors. However, the calibration is not an absolute measurement, it merely relates response at one time in one detector to that at a different time in the same detector.

The calibration is calculated on a daily basis by comparing the average response of a plane to a through-going muon over the entire detector at time $t_{0}$ to that recorded at a time $t$ :

$$
D_{\text {scint }}(t, d)=\frac{\text { Median } \operatorname{response}\left(d, t_{0}\right)}{\text { Median } \operatorname{response}(d, t)}
$$

\subsubsection{Strip-to-Strip Non-Uniformity Calibration}

Though going cosmic muons are also used to calibrate the strip-to-strip time dependent response of the detectors $S(s, t, d)$. In both detectors the strip ends have shown deviations of light output up to $\sim 30 \%$ [76]. The goal is to calibrate out these deviations on a strip by strip basis to ensure the response to energy deposition is uniform across the entire detector; this calibration relates the mean response of each strip end to the entire detector average:

$$
S(s, t, d)=\frac{\text { Mean response of detector }(d, t)}{\text { Mean response of } \operatorname{strip} \operatorname{end}(s, d, t)}
$$

This calibration constant is calculated using the mean response of a muon of normal incidence traveling through the centre of a strip, thus eliminating spatial and angular dependencies, attenuation and path-length corrections. A statistical approach is taken to accommodate the Poisson nature of the PMTs; low numbers of photoelectrons $(\sim 2-10)$ arrive at the PMTs and the average calculation must take into account the probability of zero-photoelectron events $[103,104]$. 


\subsubsection{WLS Fibre Attenuation Correction}

Though-going cosmic muons can also be used to calibrate the attenuation of the light as it travels along the WLS fibres in the scintillator strips. However, a more accurate approach was to use a "module mapper" during construction of the module scintillators. The mapping device uses a well-defined $\gamma$ source produced from ${ }^{137}$ Caesium to illuminate a small section of the face of the scintillator modules. A signal is registered from the PMTs and recored every $40 \mathrm{~ms}$, every $8 \mathrm{~cm}$ along the strip. A fit with a double exponential is performed, given by

$$
A(x)=A_{1} e^{-x / L_{1}}+A_{2} e^{-x / L_{2}},
$$

where $x$ is the position along the strip, $L_{1}$ and $L_{2}$ are the two attenuation lengths, and $A_{1}$ and $A_{2}$ are the attenuation constants. The attenuation constants were cross-checked using a high statistics sample of through-going cosmic muons from the ND. The pulse height from a strip hit by a track is plotted as a function of the longitudinal track position; the agreement between this and the double exponential fit is to within $\pm 4 \%$ as shown in figure 4.1 . Small variations in the light output originate from small differences between each scintillator such as fibre depth, impurities, and the behaviour of the glue in construction.

\subsubsection{Inter-Detector Calibration}

Before the conversion to absolute energy deposition in $\mathrm{GeV}$ a final scaling factor must be applied. This factor is called a Muon Energy Unit (MEU). This scaling factor serves to normalise the response from a scintillator plane from one of the detectors to all planes in all detectors. Cosmic muons are used due to their abundance in the detectors since it is possible to determine a muon's momentum independently without the use of calorimetry. MINOS has two methods for measuring muon momentum: muons that stop inside the detector for which 


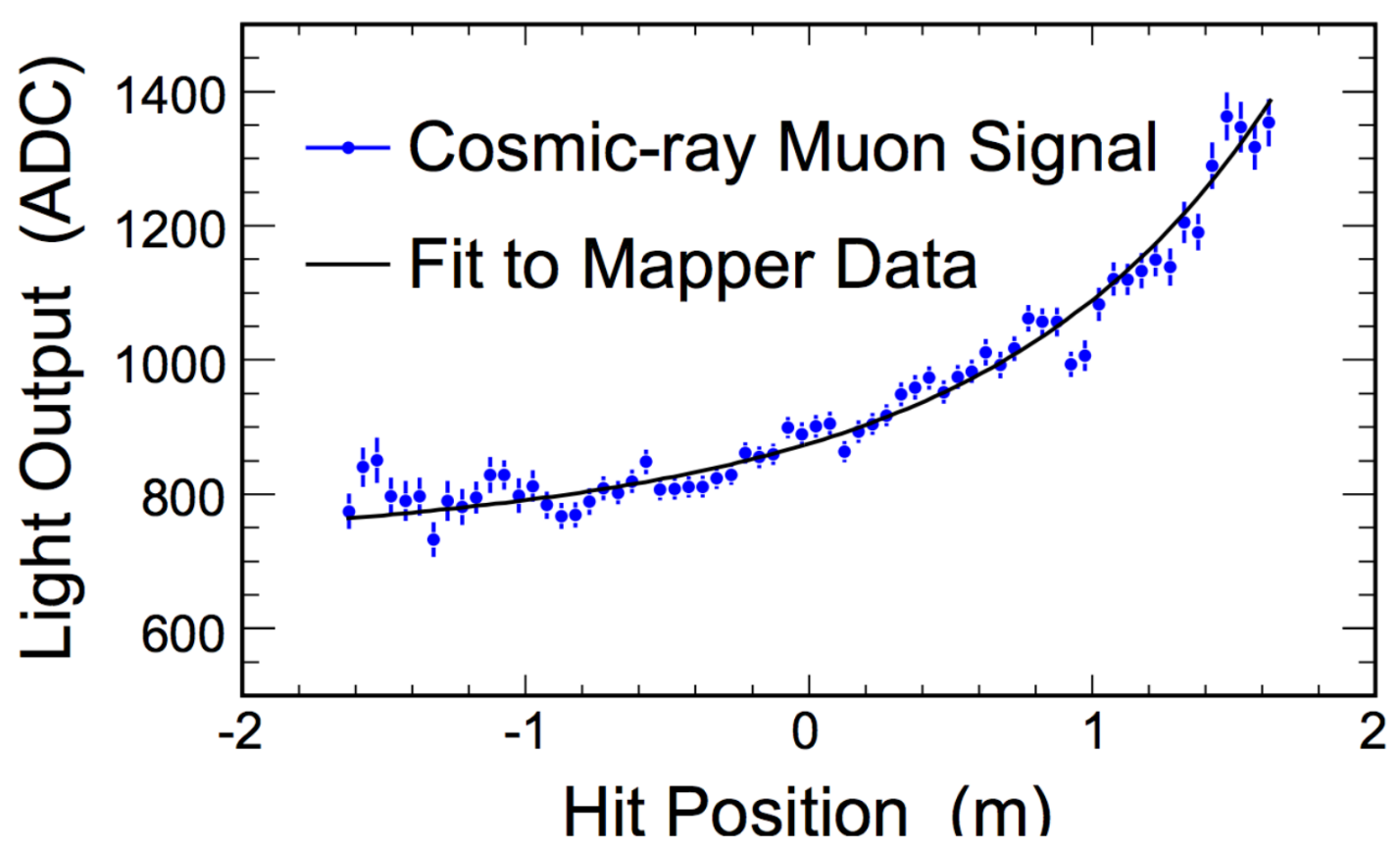

Figure 4.1: Cross-check between cosmic ray muon data (points) and modulemapper fitting results using a double exponential fit (solid curve) for a typical strip in the Near Detector. Figure taken from [76].

their range is the best measure and muons that pass straight through a detector for which the curvature of the track due to the magnetic field is used. MINOS uses stopping cosmic muons (muons stopping within the detector) to calculate the inter-detector calibration because knowledge of the total energy deposited is required.

The energy loss of a muon in copper is described by the Bethe-Bloch equation [28], which describes the rate of energy loss as a muon travels through a medium as shown in figure 4.2. The energies of the cosmic muons used for the inter-detector calibration range from $1-200 \mathrm{GeV}$ making them minimally ionising particles (MIPs). This corresponds to the flat linear region in figure 4.2 and gives an almost constant energy deposition per plane in the detectors.

For the inter-detector calibration the total energy deposited by the muon has to be known. The simplest method to calculate the total energy deposited would be to sum the total pulse height per plane for stopping muons in the detectors. 


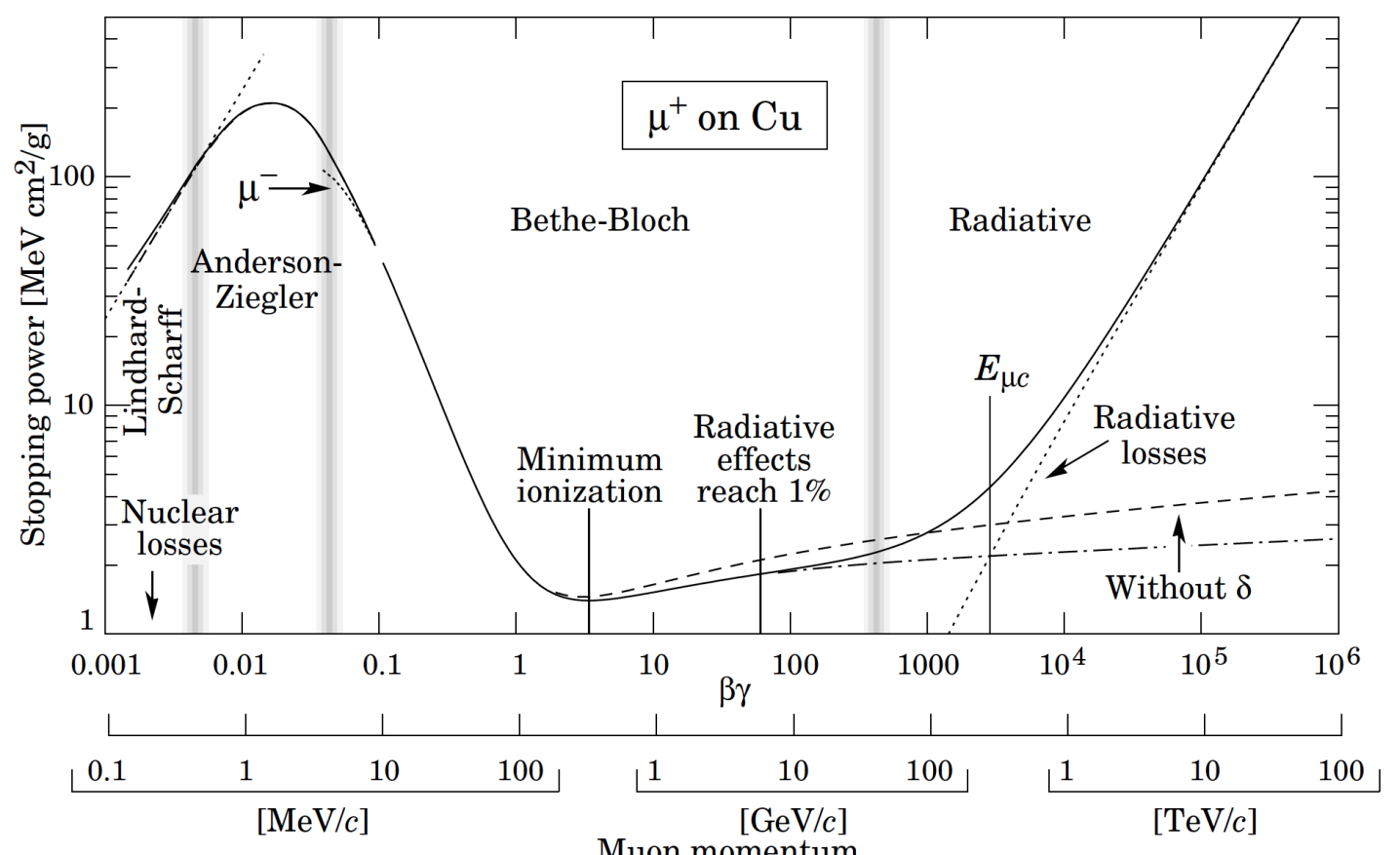

Figure 4.2: The stopping power of muons in copper as a function of muon momentum. The region important in MINOS is the region between $0.5-1.1 \mathrm{GeV}$. Figure taken from [28].

However, a muon with low momentum $<0.5 \mathrm{GeV} / c$ (corresponding to the end of the muon track) undergoes non-linear energy loss $(d E / d x)$ due to a rapid increase in ionisation from the muon [105]. Muons with larger momenta (the beginning of the muon track) can emit large amounts of bremsstrahlung radiation resulting in non-linear energy deposition. A more robust technique is used to calculate the total energy deposited by stopping cosmic muons. Muons with momenta in the range $0.5-1.1 \mathrm{GeV}$ are used; this corresponds to looking at the "track window" [99]. Figure 4.3 shows a simulated cosmic muon with an initial energy of $1.8 \mathrm{GeV}$; the track window used for calibration is highlighted. Within this track window the cosmic muon has a momentum between 0.5 and $1.1 \mathrm{GeV}$; this flat region results in a reasonably constant $d E / d x$ across planes.

Through the use of the "track window" the $d E / d x$ varies so slowly in the 0.5 - $1.1 \mathrm{GeV} / \mathrm{c}$ region that a $2 \%$ error on knowing where the muon stopped yields an uncertainty on the MEU of only $0.2 \%$ [99]. This final calibration constant will 


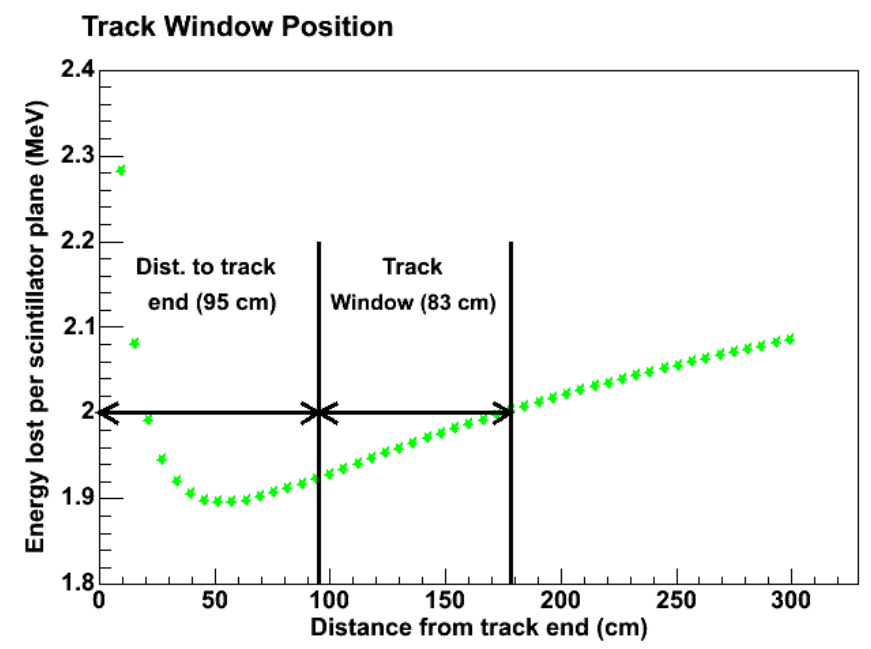

Figure 4.3: True energy lost per scintillator plane for a simulated cosmic muon with initial energy of $1.8 \mathrm{GeV}$ and the window in track length used for calibration. Figure taken from [99].

calibrate the detectors so that a known particle of known energy passing through a plane of scintillator in any of the MINOS detectors will give an identical response to within the errors of the calibration. This is under the assumption that the thickness of all the scintillators and steel planes are the same throughout all the MINOS detectors; this is not exactly true, for example, the first 190 planes in the FD are approximately 1\% thicker than the remaining 296 planes [106]. These small deviations from uniformity are accounted for in the calibration with additional correction factors.

\subsubsection{Summary of Calibration Chain}

Figure 4.4 shows the response for both the ND and FD before, during and after calibration. By performing this calibration the overall response of the detectors is uniform. This is critical as biases or systematic shifts between detectors can lead to incorrect measurements with large errors when measuring the energydependent oscillations. 

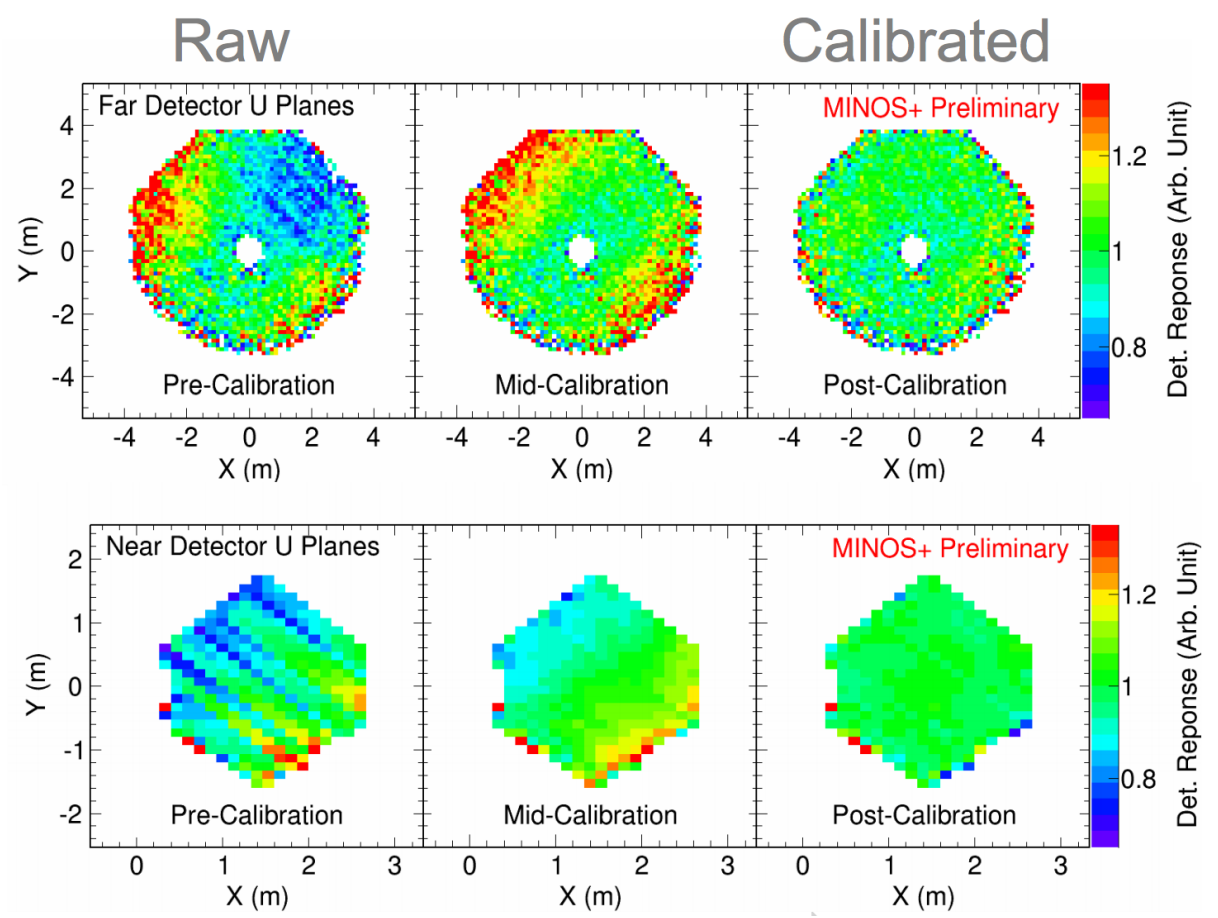

Figure 4.4: The response of the Near (bottom panel) and Far (top panel) Detectors. The first column is the detector response before the calibration procedure is performed, the middle column is part-way through the procedure and the final column is once the calibration procedure has been completed.

\subsubsection{Absolute Energy Scale}

After the calibration chain discussed above, the detectors exhibit a uniform response to hadronic and electromagnetic showers. However, it is still necessary to convert that response into a measure of the deposited energy. This requires a suitable source of hadrons and electrons, which is not available at the MINOS ND and FD. The CalDet detector described in section 3.12 was exposed to beams of $p^{ \pm}, \pi^{ \pm}, e^{ \pm}$, and $\mu^{ \pm}$in the energy range of $0.4-10 \mathrm{GeV}$ to establish the response of the detectors to hadronic and electromagnetic interactions of known energies and the event characteristics in the detector. More details of the results can be found in [105].

The typical calorimetric response to pions and electrons from the test beam can be seen in figure 4.5 compared to simulations. The observation of muon interactions from a test beam in CalDet have shown that muon track energy can 


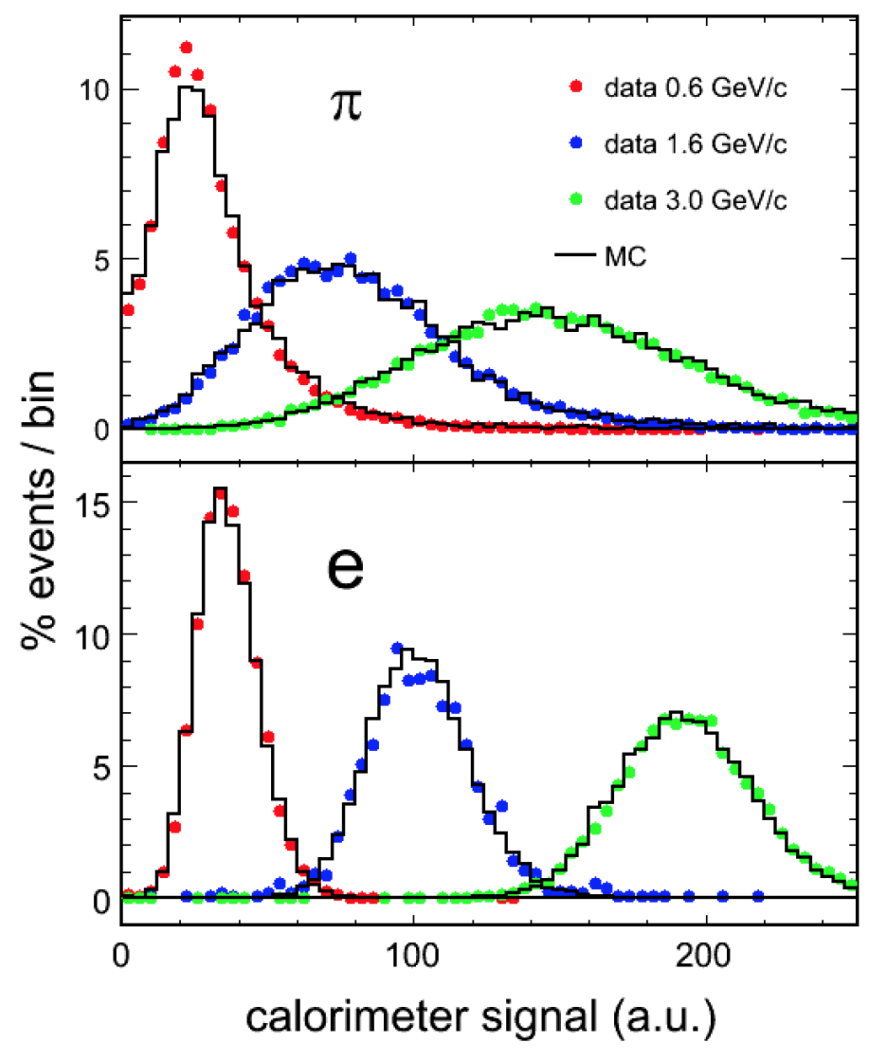

Figure 4.5: Calorimetric response of the MINOS Calibration Detector (CalDet) to pions and electrons at three different momenta from the test beam. Both simulated and real data is shown. Figure taken from [76].

be measured by range to within $2 \%$. Using the data from the hadronic [107] and electromagnetic [108] showers inside CalDet the calorimetric shower energy resolution is known as $55 \% / \sqrt{E}$ for hadrons and $20 \% / \sqrt{E}$ for electrons.

\subsection{Simulation Software}

Simulation software plays an important role in experimental measurements. It can be seen as the link between theoretical calculations experimental data. Simulation generators have to simulate a wide range of physics. It begins with protons of known energy incident on a target. Additional steps are then simulated leading to a neutrino that interacts within a detector with a target particle or nucleus. Not only does the final state of this interaction need to be simulated but all poten- 
tial re-interactions within the nucleus that could produce a plethora of different particles over a wide range of energies must also be simulated. It is the use of these simulations that link reconstructed variables to true variables in an experimental analysis. There is no single software package that can simulate everything; instead a multitude of packages are used to simulate the physics at MINOS. From the interaction of the proton beam incident onto the graphite target to propagation and interactions of neutrinos as they travel toward and interact within both detectors, this requires a chain of various software packages that will be described below.

\subsubsection{Simulating the NuMI Beam}

MINOS simulates the neutrino beam using a Monte Carlo (MC) generator called FLUGG [109, 110]. This incorporates two packages: GEANT4 [111] which provides a geometry simulation of the NuMI target and beamline and FLUKA [112] which uses the GEANT4 geometry to simulate the hadron production of the $120 \mathrm{GeV}$ protons incident on the graphite target. It records and models the resulting mesons, which are focused by the focusing horns into a decay pipe where the boosted hadrons will decay into neutrinos (or absorbed by the material at the end of the pipe).

To save on processing time the nature of the isotropic decay of the parent mesons into neutrinos is used (muon decay is more complicated but is also simulated). Using the information of the position and momentum of the parent the daughter neutrino is "weighted" such that the neutrino event is forced to travel into the detector or surrounding rock. This method allows for a signifiant number of events to be simulated on a reasonable time scale, eliminating neutrinos that would not pass through either of the detectors. This is particularly useful for simulating events at the MINOS FD which only occupies a small solid angle viewed from the decay pipe. 


\section{SKZP Weights}

Beam simulations do not well model the observed neutrino flux due to the large uncertainties on hadron production in long targets. The two-detector setup at MINOS was intended such that the ND would experience no neutrino oscillations and would measure an observed energy spectrum that can be used to characterise the neutrino energy spectrum delivered from the NuMI beam facility.

It is useful to describe hadron production models in relation to the longitudinal momentum component (along the beam axis), $p_{z}$, and the transverse momentum component (perpendicular to the beam axis), $p_{t}$, of the system with a centre of mass energy $\sqrt{s}$. The scaling variable, $x_{f}=2 p_{z} / \sqrt{s}$, proposed by Richard Feynman [113], will become convenient to use when calculating the hadron production uncertainties discussed later in chapter 6 .

By comparing the energy spectra from ND data to those from simulated beam $\mathrm{MC}$ over a range of different energy configurations (to cover a range of $p_{t}-p_{z}$ space) in both neutrino and anti-neutrino modes, discrepancies between data and MC are reduced through applying weights to the spectrum of the neutrino parents generated such that deviations as large as 30\% in the energy tail are corrected for. These weights are known as $S K Z P$ weights; more details on this procedure can be found in reference [114]. Figure 4.6 shows the MC at the ND before and after being reweighed using this procedure.

It is important to note that these SKZP weights are derived under the assumption that the ND observes no neutrino oscillations and therefore the energy spectra it measures are a true representation of the neutrino beam produced. This is true assuming a three-flavour model where the atmospheric mass splitting $\Delta m_{32}^{2}$ is of order $10^{-3} \mathrm{eV}^{2}$ and the solar mass splitting $\Delta m_{21}^{2}$ is of order $10^{-5} \mathrm{eV}^{2}$. However, for this thesis where a sterile neutrino mass splitting is introduced, a wide range of values for $\Delta m_{41}^{2}$ are investigated. For values of $\Delta m_{41}^{2} \sim 1 \mathrm{eV}^{2}$ and above, neutrino oscillations occurring at the MINOS ND need to be accounted 

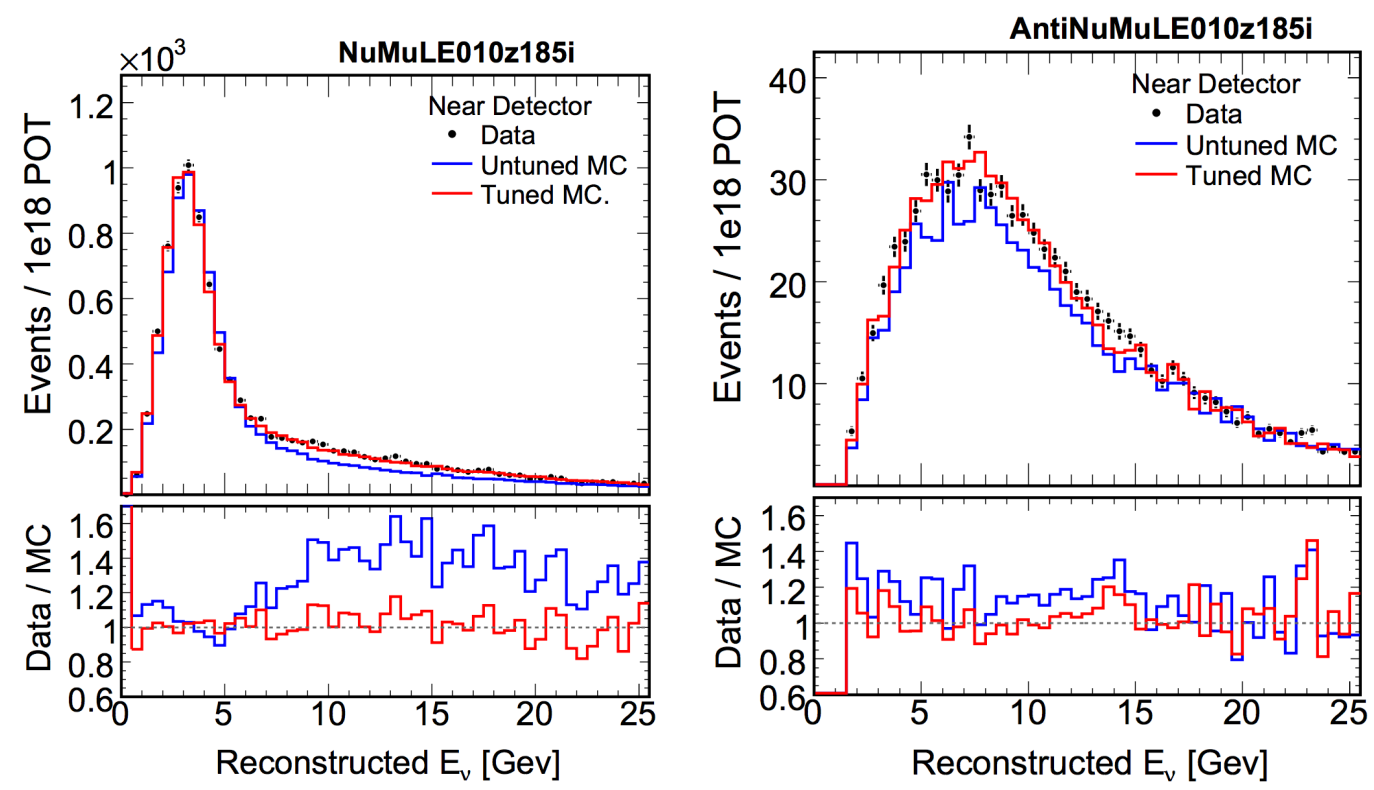

Figure 4.6: The Near Detector reconstructed neutrino energy spectra for data and simulation with and without beam reweighing. Figure taken from [115].

for. Therefore great care is taken to explicitly state when these weights are applied in plots presented throughout this thesis. The fitting procedure in the main analysis will not use these weights at all. Only when comparing data to threeflavour distributions are the SKZP weights applied. Since the weights are not applied in the main analysis an appropriate systematic uncertainty is included on the neutrino flux, which will be discussed in chapter 6 .

\subsubsection{Simulating the MINOS Detectors}

The neutrino interactions inside the MINOS detectors are simulated by $N E U$ GEN [116], which simulates elastic, quasi-elastic and inelastic neutrino interactions. The quasi-elastic interactions are modelled using the Llewellyn-Smith model [117] and resonance interactions modelled with Rein-Seghal [118, 119] and the modified Bodek-Yang model [120] for deep-inelastic scattering over a wide range of energies $(0.5-100 \mathrm{GeV})$. NEUGEN simulates hadronization using a combination of PYTHIA/JETSET [121] and the KNO [122] phenomenological model, this combination is known as the AGKY [123] model. NEUGEN also 
simulates any interactions of hadrons leaving the target nucleus using the $I N$ TRANUKE [124] model of internuclear scattering.

Once the final state particles have left the nucleus they are passed to the GMINOS [125] framework that takes the particles produced by NEUGEN, and using a detailed geometric map of the MINOS detectors including the magnetic field from GEANT4, the particles are propagated through the detectors taking into account any relevant physics processes using the GCALOR [126] package. The particles are propagated until their energy decreases below $10 \mathrm{keV}$ when the remaining energy is deposited into the medium (e.g. simulated scintillator or iron).

GMINOS records the energy deposited by the propagated particles on a stripby-strip basis. A C ++ program called Photon Transport then converts this deposited energy into photons that are propagated along WLS fibres to PMTs where they are converted into photoelectrons using a Poisson number generator. Photon Transport takes into account the detector response, electronics noise and non-linearity of the devices as described earlier in this chapter. Finally a program called DetSim takes the readout from the electronics and propagates it to the MINOS reconstruction software where it is treated as if it were actual data. The simulation is "de-calibrated" meaning the inverse of the calibration constants at a randomly-generated timestamp are applied in order to best simulate the raw data taken in the MINOS detectors. The randomly-generated timestamp is associated with the simulated event so that at a later time the correct calibration constants can be reapplied once the simulated data has been reconstructed in an identical procedure to that applied to real data. 


\subsection{Reconstruction}

The neutrino interactions of interest to MINOS (section 3.13.1) can be reconstructed from the patterns of energy depositions inside the MINOS detectors. An ordered set of algorithms use the raw energy deposition combined with topological and timing information to construct tracks and showers inside the detectors. It is the construction of these objects that will form the fundamental constituents for determining the energy of a neutrino event and the type of interaction it underwent in the detectors. A $\nu_{\mu}$ or $\bar{\nu}_{\mu} \mathrm{CC}$ event will contain an outgoing muon track whereas an NC interaction will leave a large diffuse hadronic shower. Figures 4.7 and 4.8 show reconstructed simulated $\mathrm{CC}$ and $\mathrm{NC}$ events; this demonstrates the final event once all the tracks and showers have been fully reconstructed. The following is a description of the procedure used to construct these quantities.

Scintillation light emitted due to the presence of energy deposition from an ionising particle is converted into charge that is read out by the electronics. The resulting pulse height is digitised and quantified into a metric called a digit. A digit is associated with a known timestamp and a list of possible scintillator strips due to the multiplexing of the strips onto the PMTs (see section 3.9).

By grouping digits with a similar timestamp together a strip is formed, characterising the energy deposition across a strip. Due to the high neutrino flux in the ND, multiple strips are formed from the numerous neutrino interactions occurring within a short time. Sets of ND strips are grouped into slices based on their locality to one another and timing information such that slices are constructed containing strips from a single neutrino interaction. This process does not have to be performed at the FD as the low flux results in less activity within this time window and therefore strips do not have to be grouped into slices. All strips in the FD trigger window can be thought as a slice. These slices are then passed to the next stage of the reconstruction, which aims to form tracks and showers out of the collection of strips. 

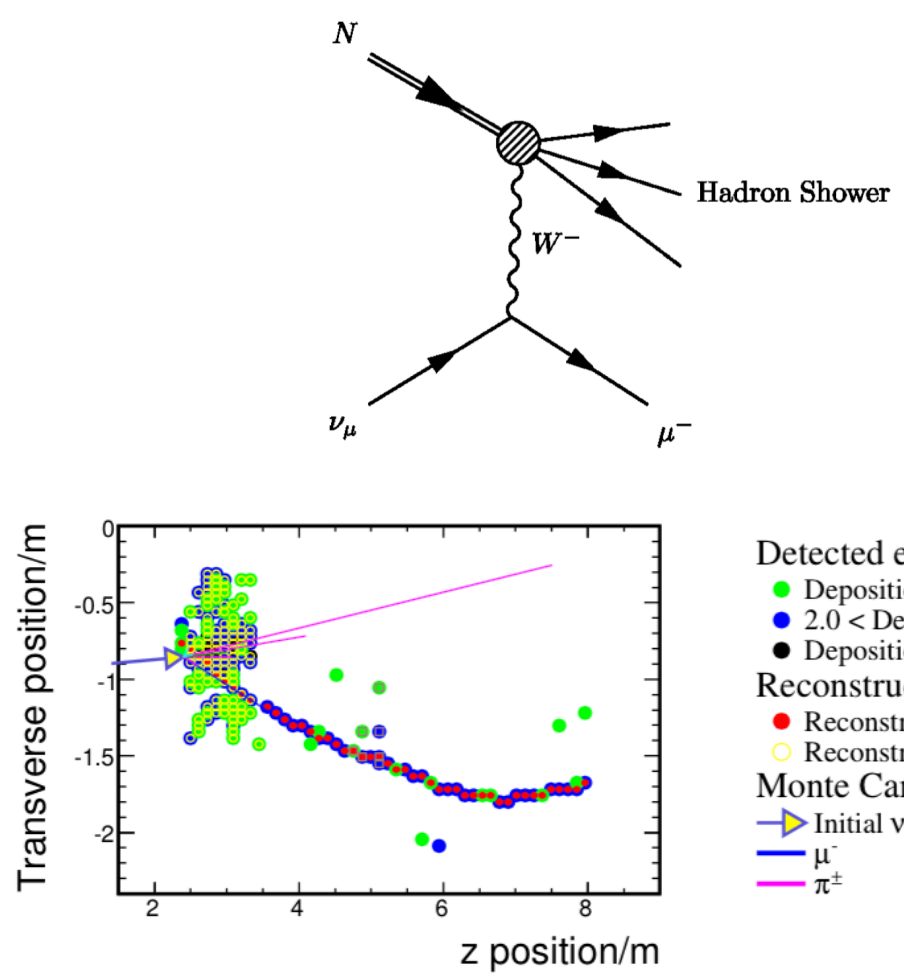

Detected energy depositions

Deposition $<2.0$ pe

- $2.0<$ Deposition $<20.0$ pe

- Deposition $>20.0$ pe

Reconstruction

- Reconstructed track hit

Reconstructed shower hit

Monte Carlo Truth

$\rightarrow$ Initial $v_{\mu}$

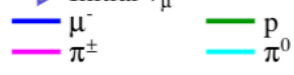

Figure 4.7: Top: Feynman diagram of a CC interaction of a $\nu_{\mu}$ with an iron nucleus. Bottom: A simulated CC event display inside the MINOS detector. The green, blue and black dots represent signals of different amounts of energy deposited in the scintillator. Red dots mean the scintillator hit has been reconstructed as part of a track. Yellow circles mean the scintillator hit has been reconstructed as part of a shower. The outgoing muon forms a long track used to identify the CC interaction. The curvature of the track is caused by the magnetic field in the detector, and is used to determine the charge sign. Figure taken from [127].

\subsubsection{Track Reconstruction}

Both $\mathrm{CC}$ and $\mathrm{NC}$ events can have track-like topologies and the process of how they are distinguished is discussed in chapter 5 .

A track-finding algorithm is applied to the strips in a slice. This algorithm searches for track-like segments from hits in neighbouring planes. A "seed track" is initially constructed by combining the most likely track segments together.

The seed track is then fitted using a Kalman Filter [128, 129], which uses a series of equations to extrapolate along the track (accounting for missing energy deposition between planes), continually estimating the momentum of the 

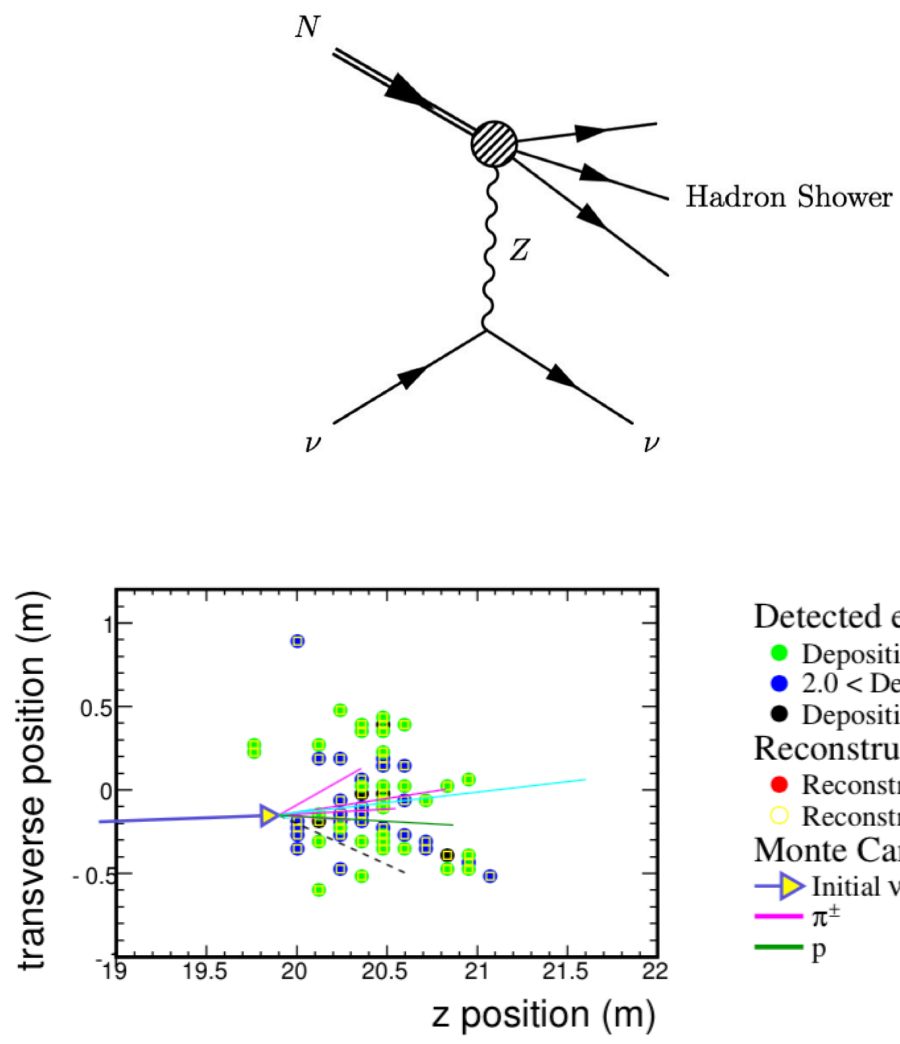

Detected energy depositions

- Deposition $<2.0$ pe

- $2.0<$ Deposition $<20.0$ pe

- Deposition $>20.0$ pe

Reconstruction

- Reconstructed track hit

Reconstructed shower hit

Monte Carlo Truth

$\begin{array}{ll}\rightarrow \text { Initial } v_{\mu} & \cdots \text { Outgoing } v \\ -\pi^{ \pm} & =\mathrm{n} \\ \mathrm{p} & -\pi^{0}\end{array}$

Figure 4.8: Top: Feynman diagram of a NC interaction between a $\nu$ and iron nucleus. Bottom: A simulated NC event display inside the MINOS detector. The green, blue and black dots represent signals of different amounts of energy deposited in the scintillator. Red dots mean the scintillator hit has been reconstructed as part of a track. Yellow circles mean the scintillator hit has been reconstructed as part of a shower. A typical $\mathrm{NC}$ event will have a wide diffuse hadronic shower. Figures taken from [127].

underlying muon as it travels through the alternating planes of scintillator and steel. The estimation of the momentum takes into account stochastic processes, electronics noise, multiple scattering and the curvature of the track due to the magnetic field. The filter decides on what segments of the original seed track to keep in order to construct a fully reconstructed track. The Kalman Filter estimates the ratio of the charge to the momentum of the muon, $q / p$, along with its uncertainty, $\sigma_{q / p}$.

The resolution of muon energies measured using curvature is $11 \%$ at $3 \mathrm{GeV}$ [105]. For tracks that end in the detector, by using the range to estimate the energy a 
resolution of $4.6 \%$ at $3 \mathrm{GeV}[105]$ is obtained.

\subsubsection{Shower Reconstruction}

The remaining hits in the detector that have not been formed into tracks are clustered together depending on their proximity to one another. Any hits already in a track have the energy deposition expected from a muon subtracted from them, and the reduced hit is then included into the clustering algorithm. Hits are only included into the clustering algorithm if they have a pulse height greater than two photoelectrons; this is to reduce the impact of poorly-understood disagreements in data/MC comparisons for small hits most likely caused by cross-talk from PMTs and noise from fibres (discussed later in section 5.1). The energy of the shower is estimated at this stage using a calorimetric approach influenced heavily by the results obtained from CalDet in the test beams at CERN.

\subsubsection{Event Building}

Once the tracks and showers have been formed an event builder is used to construct a neutrino event by grouping the relevant tracks and showers together. Both CC and NC events can contain a multitude of tracks and showers; the event builder will assign a primary track and shower to an event, and from this an event vertex can be reconstructed, usually from the first hit of the primary track [130]. The event-building procedure is relatively simple at the FD where there is usu-

ally a single event per beam spill. However, the ND is likely to have overlapping tracks and showers in both space and time from multiple neutrino interactions. A series of cuts are applied in order to significantly reduce the number of topological pathologies resulting from such overlapping events. 


\subsection{Shower Energy Estimator}

The analysis in this thesis uses both $\mathrm{CC}$ and $\mathrm{NC}$ samples although the end result is dominated by the CC sample. The total reconstructed energy of a neutrino

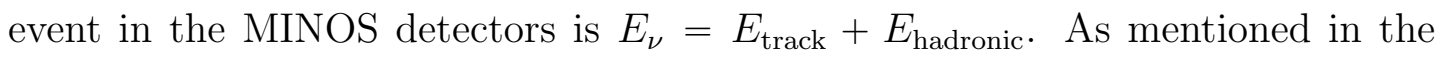
previous sections, the resolution with which neutrino energy can be measured is dominated by hadronic shower measurements through the use of calorimetry. An improvement in energy resolution would lead to a more clearly defined neutrino energy spectrum, which when used in a fit to measure the energy dependence of the neutrino oscillations from a CC sample will yield an improvement in the precision on the oscillation parameters. A multivariate technique is used for the estimation of hadronic shower energies for $\mathrm{CC}$ events in order to improve the energy resolution compared to that obtained from calorimetry.

\subsubsection{The $k \mathrm{NN}$ Algorithm}

The improved method for estimating hadronic energy for $\mathrm{CC}$ events uses the " $k$-nearest-neighbour" $(k \mathrm{NN})$ algorithm [131]. The shower energy estimation is performed such that for an observed data event, a group of the closest matching MC events (nearest neighbours) is identified using some metric. It is the averaged true shower energy of this group of MC events that is used as the reconstructed shower energy of the data event.

The distance of a data event to a training event is given by the Euclidean metric defined as

$$
\Delta s=\sqrt{\sum_{i=1}^{N} \frac{\left(y_{i}-x_{i}\right)^{2}}{\sigma_{i}^{2}}}
$$

where $y$ is the training event value for $N$ different variables such that $i=$ $\{1,2, \ldots, N\}$, and $x$ is for the observed event. For each $i^{\text {th }}$ variable the standard deviation of that distribution is taken as $\sigma_{i}$ and is used to to ensure all 
variables are treated with equal importance. The nearest 400 neighbours are used to estimate the reconstructed shower energy of the data event. The $k \mathrm{NN}$ uses $N=3$ variables:

1. The number of planes in the primary shower.

2. The calorimetric energy within $1 \mathrm{~m}$ of the track vertex.

3. The calorimetric energy in the first two reconstructed showers (if there is more than one shower).

The $k \mathrm{NN}$ energy estimator uses the same three variables at both detectors but with different MC training samples. Figure 4.9 shows the difference in energy estimation for a range of different energies compared to the traditional calorimetric approach. At higher energies both methods give similar resolutions, however at low energies the $k \mathrm{NN}$ method yields significant improvement. More detail on this $k \mathrm{NN}$ shower energy estimator can be seen found at $[129,131]$.

\subsubsection{Shower Energy Estimator Uncertainties}

The output of the $k \mathrm{NN}$ estimator will be sensitive to mis-modelling in the hadronic shower simulation. There are three main sources of uncertainty that are investigated and combined to provide a systematic uncertainty on the shower energy:

- Detector response to single particles: this size of uncertainty is determined by looking at the discrepancy between MC and data observed in CalDet in the test beam at CERN (described in section 3.12) and is 5.7\% on the calorimetric measurements [107].

- Hadronisation modelling: the uncertainty of the final state particles once neutrinos have interacted with the target nuclei. 


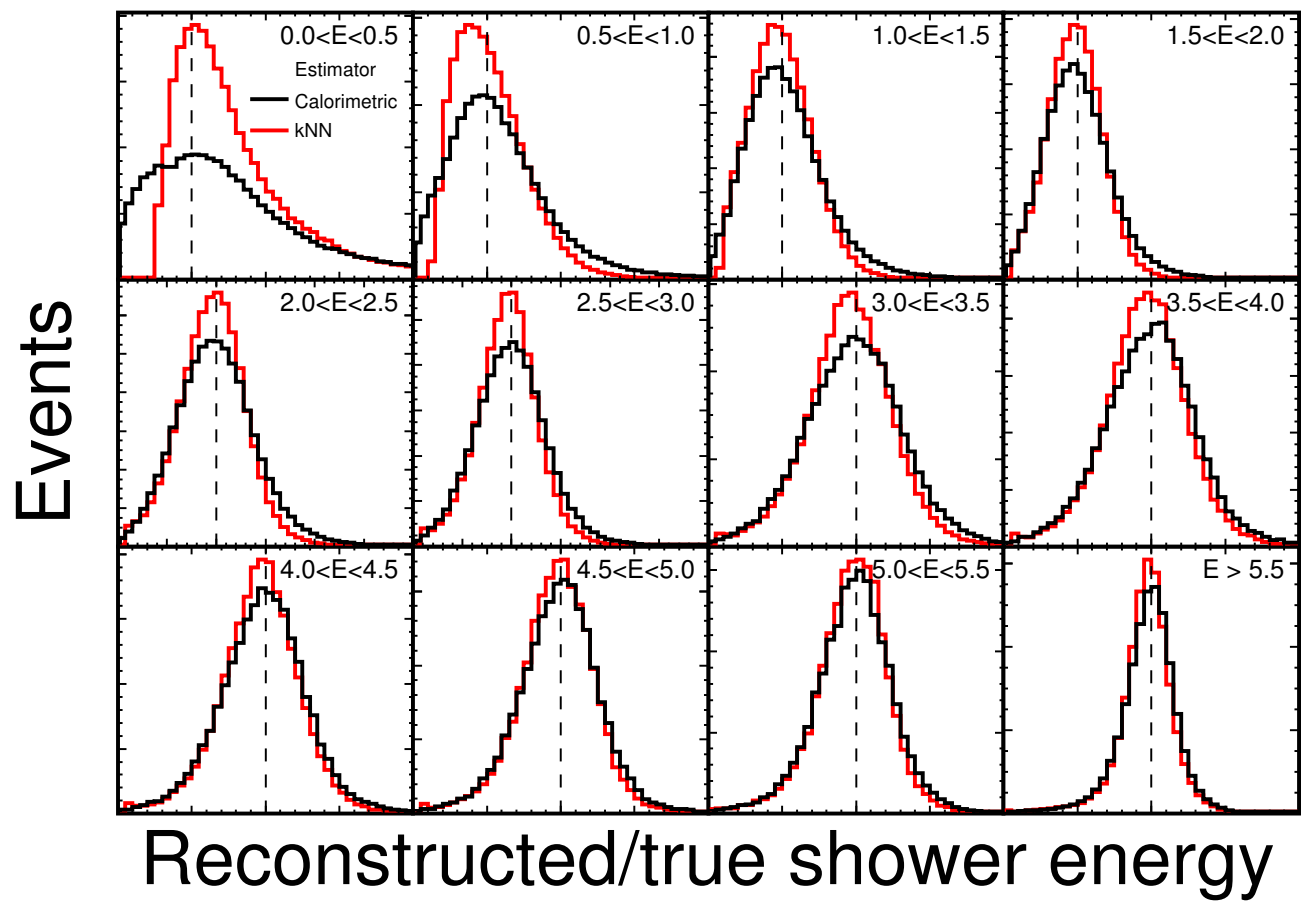

Figure 4.9: Distributions of $E_{\text {reco }} / E_{\text {true }}$ for various true shower energy ranges comparing $k \mathrm{NN}$ (red) to calorimetric (black) shower energy estimation. Figure taken from [131].

- Intranuclear rescattering: the uncertainty arising from the potential mis-modelling of hadrons before they have escaped the nucleus and are observed by the MINOS detectors.

The assessment of these uncertainties is described in detail in reference [131]. Figure 4.10 shows the total uncertainty on the $k \mathrm{NN}$ shower estimation as a function of the true shower energy compared to the uncertainty on shower energy determined through calorimetric means alone [132]. It can be seen that the $k \mathrm{NN}$ estimator has smaller systematic uncertainties at most energies. 


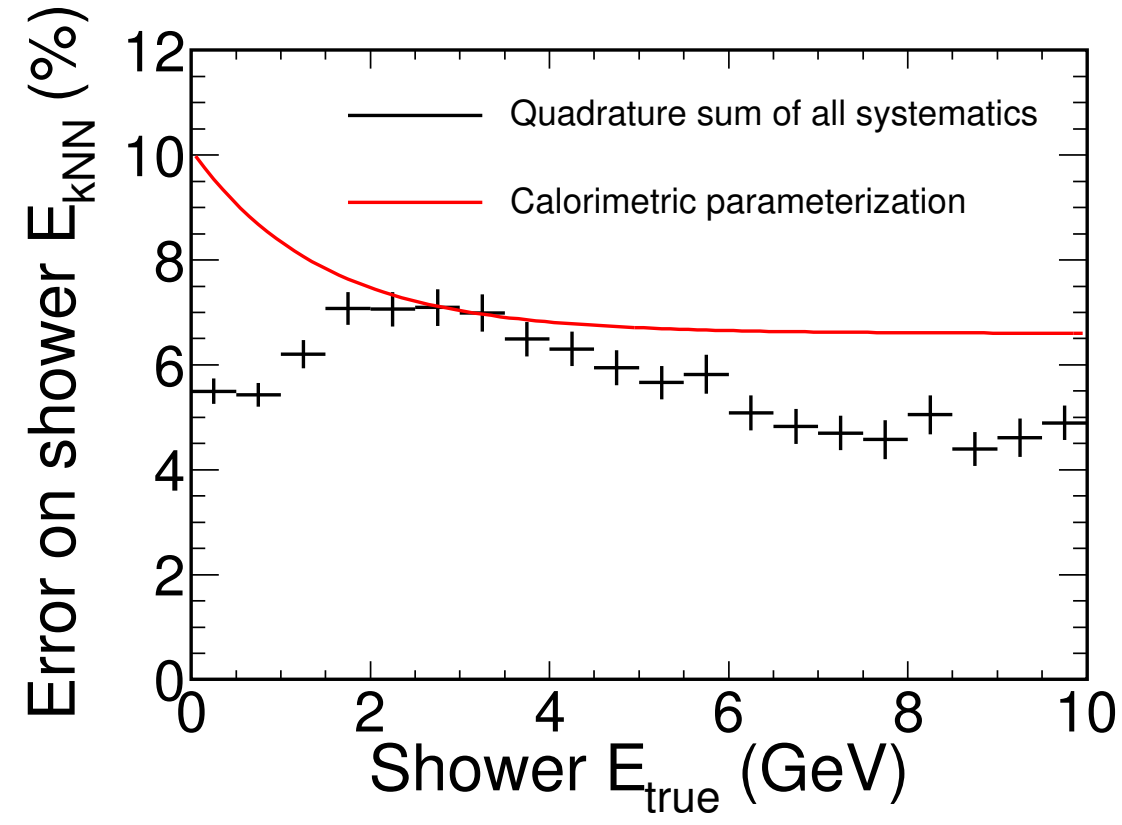

Figure 4.10: Total systematic uncertainty on the $k \mathrm{NN}$ shower energy estimator as function of true shower energy in black. The red curve is the systematic uncertainty on calorimetric shower energy. Figure taken from [131]. 


\section{Chapter 5}

\section{Event Selection}

This analysis in this thesis uses both $\mathrm{CC}$ and $\mathrm{NC}$ samples. This chapter will discuss the procedure for event selection and the efficiencies and purities of both samples. A preselection is first discussed, followed by the specifics of the NC selection for both MINOS detectors. To ensure no double counting between selectors the CC selector is performed last and selects the $\mathrm{CC}$ events from the failed NC sample to ensure both samples are exclusive. The NC selector uses a simple cuts-based method, whereas the CC selector uses a $k$ NN-based method.

\subsection{Preselection}

Preselection is an initial screening of all events that have been formed following the reconstruction, primarily to discard events reconstructed whilst the NuMI beam or detectors were not operating properly. Events that pass this selection are considered to be "good" events. They have to have good beam quality, the magnetic coil in the detectors must have been functioning well, and the detector must have been operating properly. Events are rejected if the error on the muon track momentum/charge ratio, $\sigma(q / p)$, equals $1 \times 10^{-4} \mathrm{GeV}^{-1}$; this is a value assigned to an event where the Kalman Filter failed to correctly construct the track. Any events constructed during light injection are also discarded as well as 
any events not within the beam spill window. MINOS uses a $10 \mu$ s spill window as shown in figure 5.1. Any cosmic muons interacting within the detectors during this window motivate an additional cut that removes events with $\cos \theta<0.6$, where $\theta$ is the angle between the muon track and the beam-line axis.

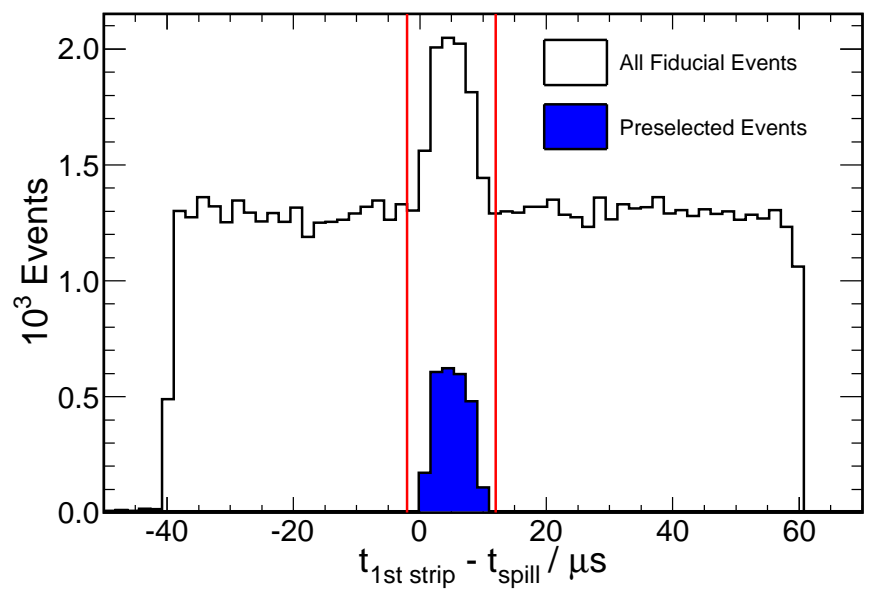

Figure 5.1: FD events as a function of the difference in time between the nearest spill to the event and the time of the first hit in the event. All events outside of the spill window indicated by the red line are discarded to reduce the background from cosmic muons. The blue distribution is the events remaining after the suite of preselection cuts, which includes cuts to remove light injection, split events, and cosmic rays. The area between the red vertical lines indicates the times between the event and the spill where an event is considered in time with the spill. This cut has been applied to the blue distribution.

The MINOS ND and FD have different electronics, detector volume and experience a different flux from the NuMI beam. Therefore both detectors have a specific set of preselection cuts to account for any inter-detector differences, which will be discussed below.

\section{ND-Specific Preselection}

Due to the increased flux the ND experiences, a preselection is performed to reduce the number of poorly-reconstructed events. A poorly reconstructed event is defined as an event with $E_{\text {true }} / E_{\text {reco }}<0.3$, where $E_{\text {true }}$ is the true energy of the event and $E_{\text {reco }}$ is the reconstructed energy. Multiple events occurring within 
the ND overlap in both space and time; this has the result of causing reconstruction failures at low energies which will primarily contaminate the MINOS NC energy spectrum since reconstruction failures tend to result in small, shower-like topologies.

An event is cut if the maximum number of consecutive planes deposited with energy is less than three. From event-scanning it is possible to observe that events with a low number of hits are poorly reconstructed [133] as shown in figure 5.2. A poorly reconstructed event will most likely have a smaller maximum number of consecutive planes.

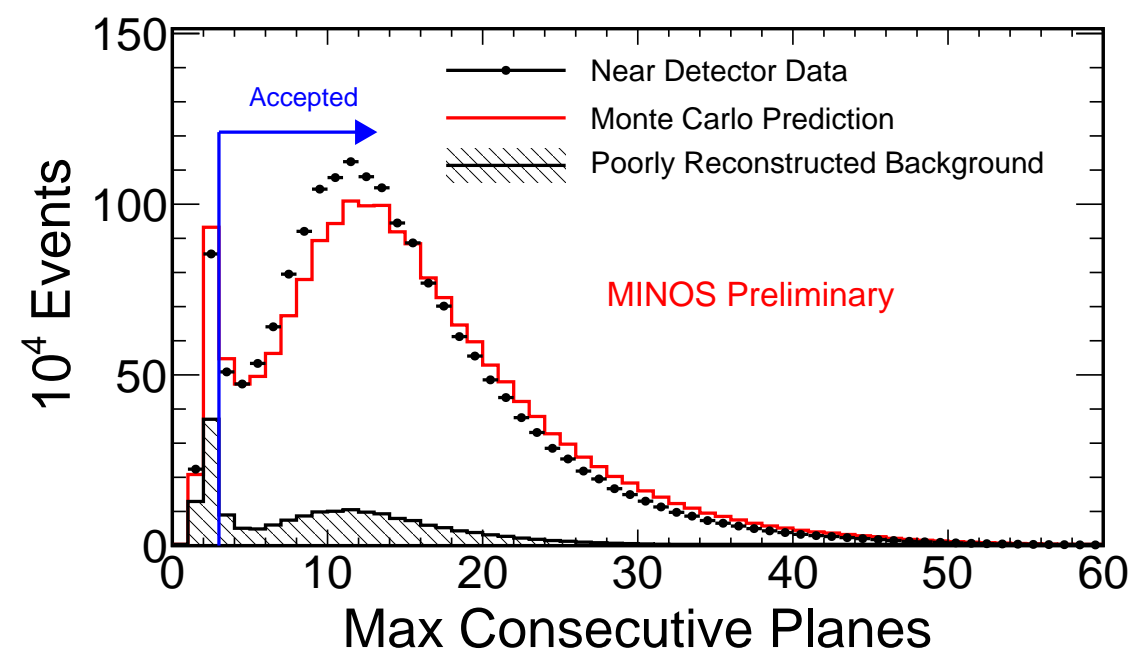

Figure 5.2: Maximum number of consecutive planes. The black points are the data, red is the total MC expectation and the hatched histogram is the number of poorly reconstructed events in the MC. Events with a maximum number of consecutive planes $<3$ are rejected. Poorly reconstructed events are included in the red histogram and give rise to the discrepancies seen, particularly at low energies.

The second cut rejects events with a pulse-height fraction $<0.5$. All hits in a beam spill in the ND are grouped into slices; a slice will contain the hits assumed to belong to a single event. The event pulse-height fraction is the ratio of the pulse-height that makes up the fully reconstructed event to the total pulseheight in the initial slice. This cut ensures that the initial slicing did a good job of isolating hits from a single, well-reconstructed event. The number of poorly- 
reconstructed events can be reduced by performing this cut [134]. Figure 5.3 shows the preselection cut for events with a pulse height fraction $<0.5$.

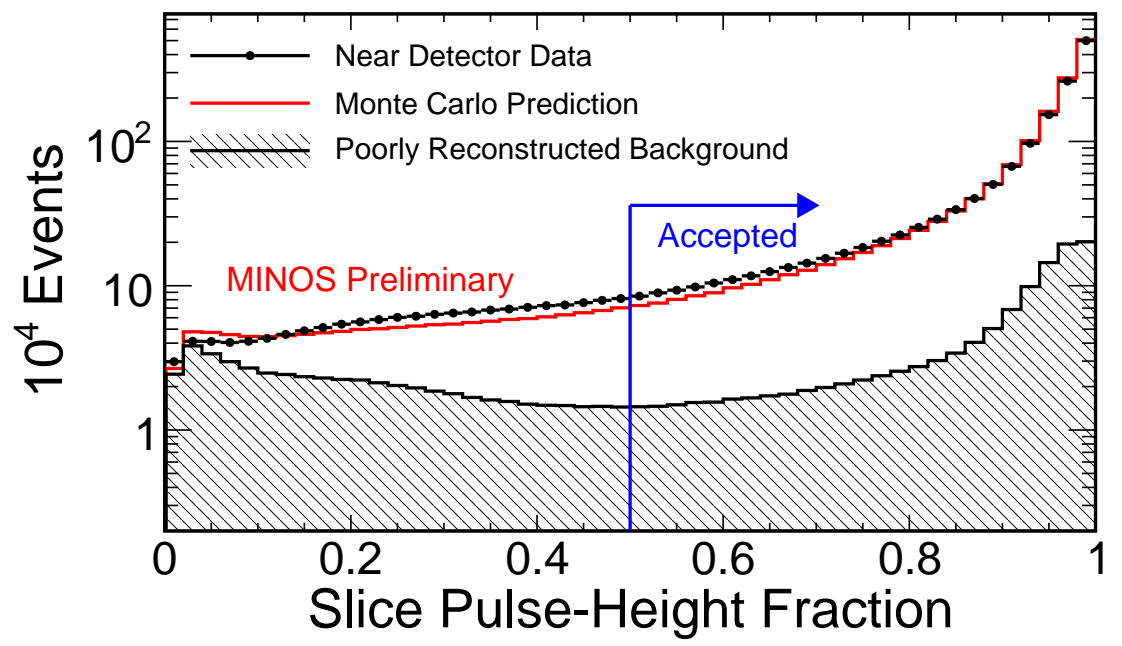

Figure 5.3: The pulse-height fraction at the ND. The black points are the data, red is the expected total MC at the ND. All events with a pulse-hight fraction $<0.5$ are rejected.

\section{FD-Specific Preselection}

The FD preselection is aimed at reducing electronics noise, the cosmic muon background, and eliminating fake events created by the light injection system.

When an LI run is performed in the FD, a signal is sent from a PMT known as a "trigger PMT". Any events reconstructed in this time can be rejected by the presence of this signal; however, if the PMT fails to send a trigger, a contamination of fake events could pass event selection. The PMT might fail to trigger due to low-powered LI pulses that are below the threshold of the PMT. These LI events will have distinct properties from a signal event and so a set of simple cuts is implemented to eliminate any potential fake events from the final sample. The LI system sends pules of light to the end of the scintillator strips; this is done at one particular end, therefore a large energy deposition would be seen relative to the other side causing an asymmetry. A real signal event will 
most likely be near the centre of the detector and due to the isotropic emission of light the asymmetry would be lower. Also, LI pulses typically have a much larger energy than the energy deposition from a MIP or hadronic shower going through the detector. An event is rejected if the following conditions are met:

- The summed pulse height from either side of the detector exceeds $1.7 \times$ $10^{6} \mathrm{ADC}$ or the asymmetry between the summed pulse-height from each side of the detector exceeds 0.55 .

- The event has more than $80 \%$ of strips in which the energy has been deposited at a single strip end.

Another potential source of noise at the FD is known as "fibre noise". That is noise due to spontaneous emission of light from the scintillator or WLS fibres, or noise from the PMTs or the electronics. Typically noise has a lower pulse-height than a MIP depositing energy within the detector. An event is rejected from the sample if the following conditions are met:

- Number of hit strips $\leqslant 8$ and pulse height $<3750$ ADCs.

- Number of hit strips $>8$ and pulse height $<2000$ ADCs.

The cosmic-ray background at the FD is suppressed by the large overburden of rock and is a sub-dominant background. A steep cosmic ray muon can go through the detector, leave no energy deposition in the veto shield and could be reconstructed as a neutrino event. This would give rise to a very tall, thin shower in the detector. A description of the cuts used to reduce the cosmic-ray background at the FD can be found in [78]. 


\subsection{NC Selection}

\subsubsection{NC Acceptance}

The NC selection uses cuts to determine if an event is NC-like or not. Firstly an acceptance cut is performed; this is a cut to provide good containment for events in a fiducial volume. Events that deposit some of their energy outside of the detector will decrease the overall energy resolution as well as increase any data/MC discrepancies due to the reconstruction algorithm having to rely on only partial event information. There are two main categories of events that deposit a fraction of their energy outside of the detector:

- Non-contained events: These are events with a reconstructed event vertex inside the detector (interacts within the detector) but the event partially develops outside of the detector. The energy of $\mathrm{NC}$ events is determined through calorimetry and therefore any energy deposited outside of the detector is not recoverable.

- Leak-in events: Leak-in events interact outside the detector but deposit some energy inside the detector; this can cause the reconstruction algorithm to mis-reconstruct this as a whole event fully contained inside the detector assigning it a fake event vertex inside the detector.

To reduce leak-in events and non-contained events all event are required to have an event vertex far enough away from the edge of the detector planes and an event $z$-vertex not outside of the detector or too close to the ends of the detector.

At the ND the fiducial volume is defined as $0.5 \mathrm{~m}$ away from the edges of the $U$ and $V$ planes (this includes the coil hole) and an event $z$-vertex requirement of $1.7 \mathrm{~m}<z<4.7 \mathrm{~m}$ where $z=0$ is the very first plane. Figure 5.4 shows this fiducial volume for the NC events. 


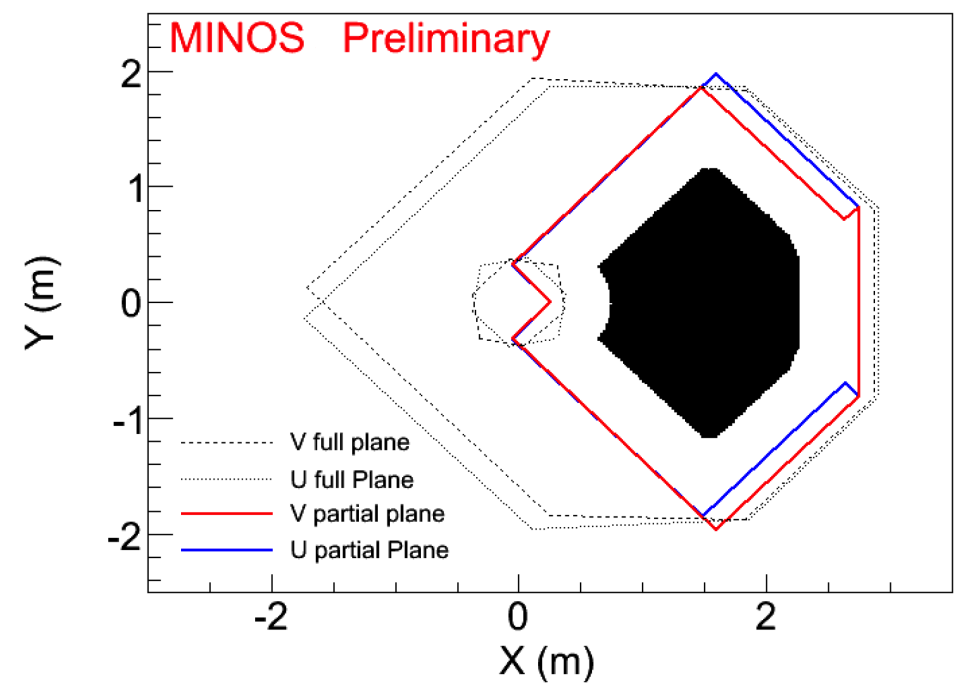

Figure 5.4: The grey area shows the ND fiducial volume for NC events. The neutrino beam is directed such that neutrinos are travelling towards the reader.

At the FD the fiducial volume is different due to the size and flux of the detector. The fiducial volume is defined as $0.4 \mathrm{~m}$ from the edges of the $U$ and $V$ planes, $0.6 \mathrm{~m}$ from the coil hole, and a $z$ vertex requirement of $0.21 \mathrm{~m}<z<$ $13.72 \mathrm{~m}$ and $16.12 \mathrm{~m}<z<28.96 \mathrm{~m}$ for the two FD super-modules. Figure 5.5 shows this fiducial volume in the $x y$-view as well as the contained vertices of NC events at the FD.

\subsubsection{NC Topology Cuts}

The following selection cuts are used to identify if an event is NC-like and reduce the CC background that would contaminate an NC sample. The NC selection cuts are identical in both ND and FD due to their design similarities. The NC selector uses a simple cut method to accept and reject events based on certain topologies expected of NC events.

An NC event will have a shower of hadronic activity; this shower will develop longitudinally and typically deposit energy in successive planes. An NC event will not deposit energy over as many planes as a $\mathrm{CC}$ event, which usually includes a long track produced by a muon. Therefore an event is rejected from the NC 


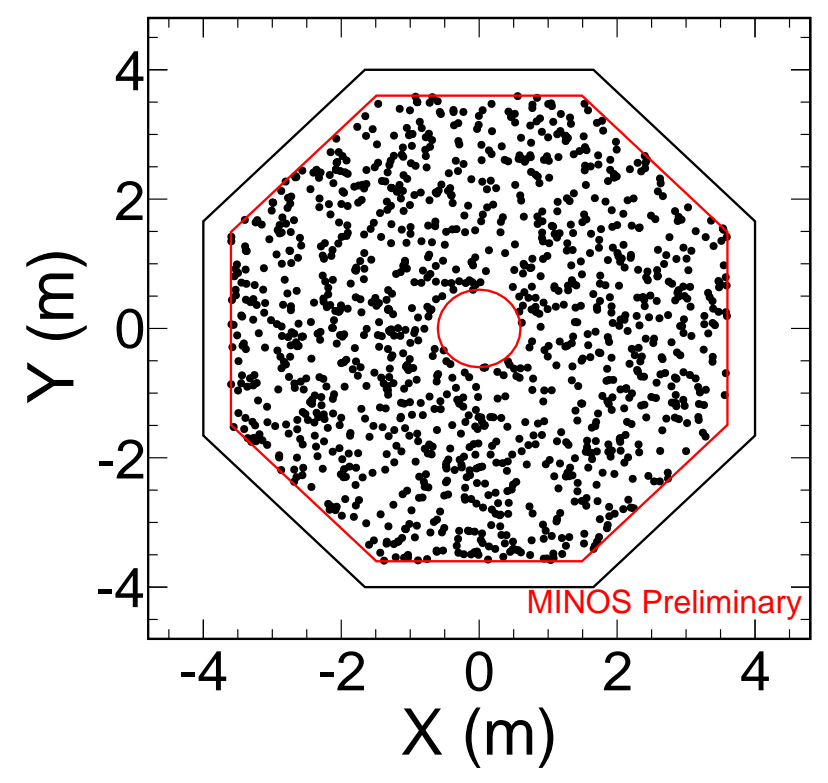

Figure 5.5: The red lines show the FD fiducial volume for NC events. The black points are the event vertices accepted by the selector.

sample if the energy-deposition range is over more than 47 planes. Figures 5.6 and 5.7 show the ND and FD events as a function of total number planes; the accepted sample is indicated by the arrow.

It is common for $\mathrm{NC}$ events to have tracks that are produced by hadrons, typically protons or charged pions. Unlike a muon track from a CC event, the length of a track from an $\mathrm{NC}$ event will be relatively short in comparison to the length of the shower in the detectors. The idea is to use the property of the relative length of the shower compared to that of the track, defining a trackextension metric. The track extension is the number of planes the track extends past the end of the hadronic shower. An event is rejected from the NC sample if the track-extension is greater than 5 planes. Figures 5.8 and 5.9 show the NC selector cuts for track extension in the the ND and FD respectively.

The value of the variables used in the $\mathrm{NC}$ selection cuts were determined by using the numbers that optimised both the purity and efficiency of the NC sample. The performance of the NC selector is quantified in section 5.4. 


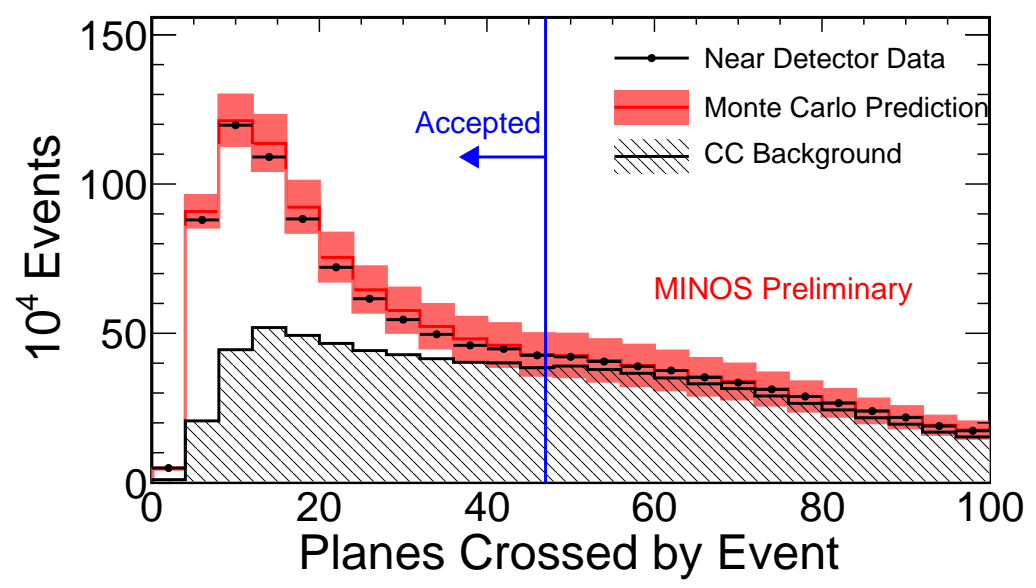

Figure 5.6: Event-length cut at the ND for neutral-current events. The red band is the uncertainty on the $\mathrm{MC}$ for the $\mathrm{ND}$, and the grey hatched histogram is the predicted CC background. The events selected are to the left of the blue arrow.

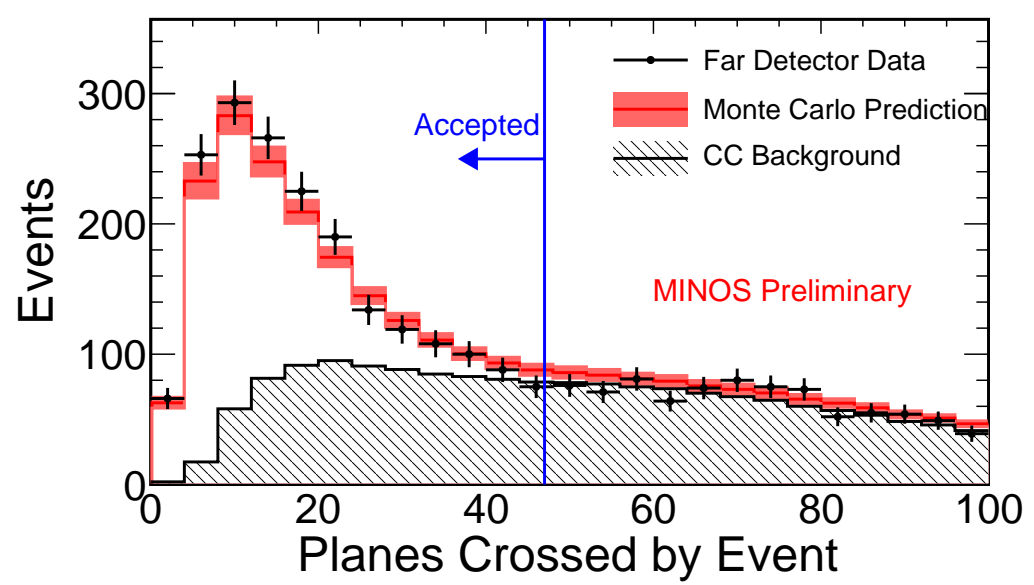

Figure 5.7: Event-length cut at the FD for neutral current events. The red band is the uncertainty on the $\mathrm{MC}$ for the $\mathrm{FD}$, and the grey hatched histogram is the predicted CC background. The events selected are to the left of the blue arrow. 


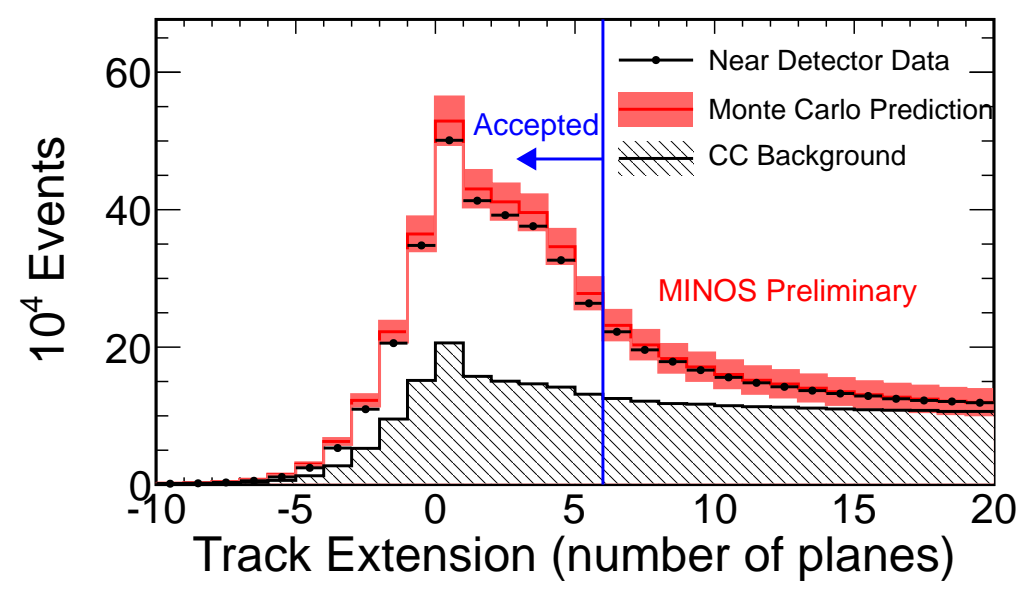

Figure 5.8: Neutral current track extension cut at the ND. The red band is the uncertainty on the $\mathrm{MC}$ for the $\mathrm{ND}$, and the grey hatched histogram is the predicted CC background. The events selected are to the left of the blue arrow.

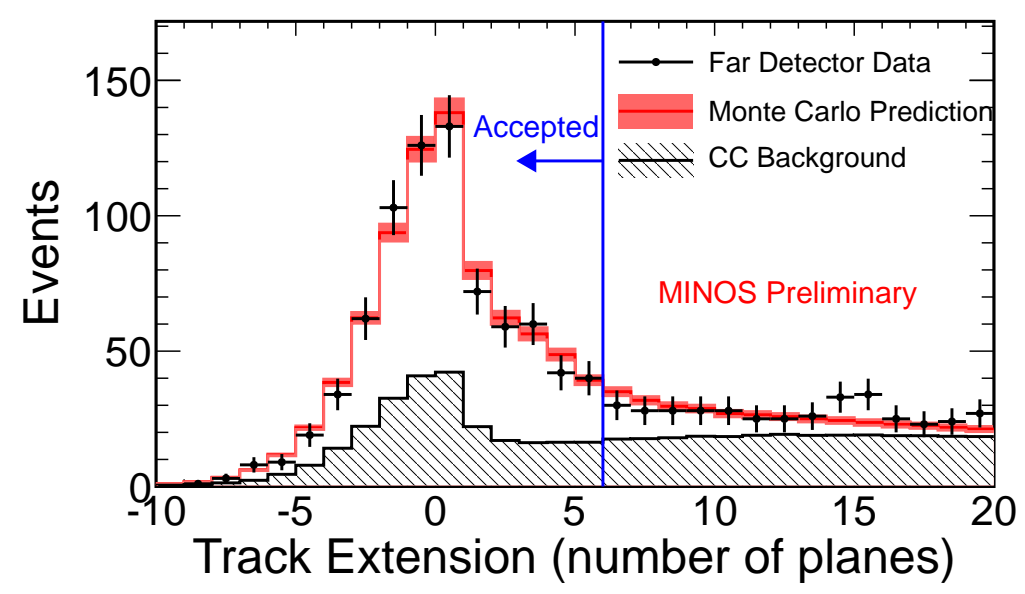

Figure 5.9: Neutral current track extension cut at the FD. The red band is the uncertainty on the $\mathrm{MC}$ for the $\mathrm{FD}$, and the grey hatched histogram is the predicted CC background. The events selected are to the left of the blue arrow. 


\subsection{Selection}

The $\mathrm{CC}$ selector is run after the $\mathrm{NC}$ selector. Any events that fail both are discarded from this analysis. The first requirement is that a $\mathrm{CC}$ event must contain a track. All events with a track are determined to be CC-like or not using a $k \mathrm{NN}$ approach. The $k \mathrm{NN}$ multivariate algorithm was described in section 4.4.1.

\subsubsection{Acceptance}

Traditionally the NC and CC selector were designed by different analysis groups and therefore defined slightly different fiducial volumes. The CC fiducial volume is a cylinder for both the ND and FD.

At the ND the fiducial volume can be seen in figure 5.10 for the CC events. The centre of the fiducial volume in each plane is centred at the neutrino beam centre $\left(x_{0}=1.48 \mathrm{~m}\right.$ and $\left.y_{0}=0.238 \mathrm{~m}\right)$ with a radius of $R=0.8 \mathrm{~m}$. An event $z$ vertex must be within the range $0.81 \mathrm{~m}<z<4.08 \mathrm{~m}$.

The coil hole is not well modelled by simulation causing large data/MC discrepancies at the ND. Therefore a cut is implemented such that a CC event is rejected if the muon track ends in the spectrometer and less than $0.6 \mathrm{~m}$ from the coil-hole centre.

At the FD the fiducial volume can be seen in figure 5.11 for the $\mathrm{CC}$ events as well as the contained vertices of CC events. The FD fiducial volume is also cylindrical in shape with a volume defined as $0.5 \mathrm{~m}<R<3.74 \mathrm{~m}$ where $R$ is defined relative to the central axis of the detector and the low bound accounts for the FD coil hole at the centre of the detector. An event is accepted if the event $z$ vertex is within the ranges $0.49 \mathrm{~m}<z<14.29 \mathrm{~m}$ and $16.27 \mathrm{~m}<z<27.98 \mathrm{~m}$ for each FD super-module. 


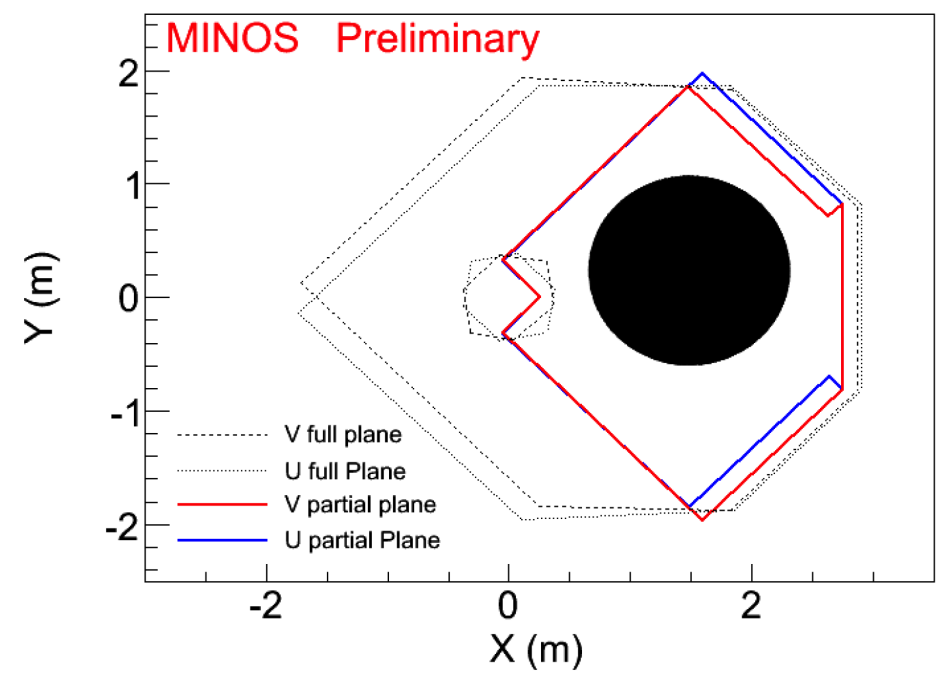

Figure 5.10: The grey circle is the ND fiducial volume for CC events. The neutrino beam is directed such that neutrinos are travelling towards the reader.

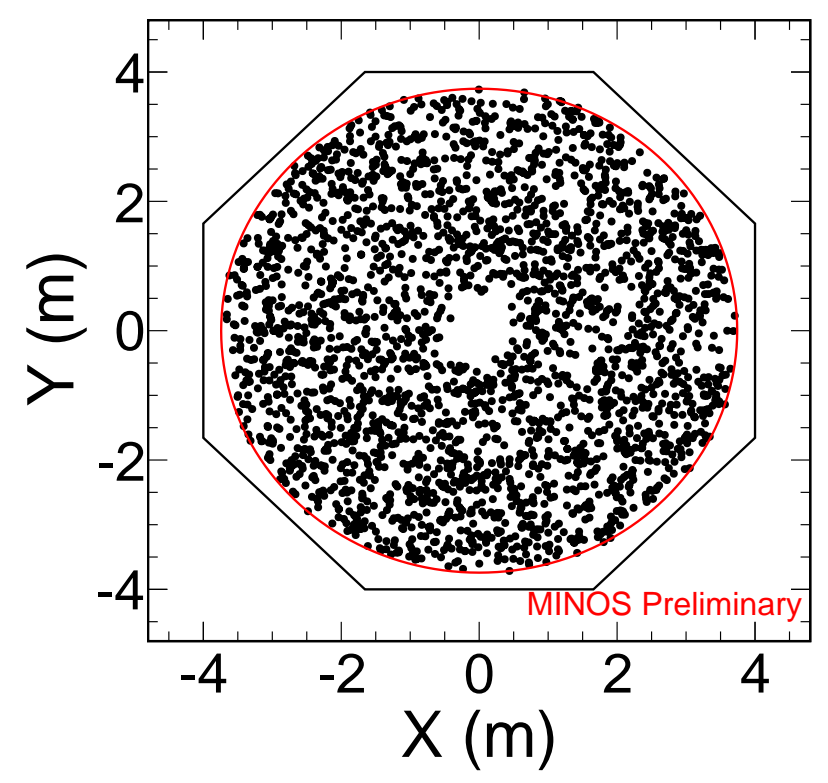

Figure 5.11: The red lines show the FD fiducial volume for CC events. The black points are the event vertices accepted by the selector.

\subsection{2 $k$ NN-Based CC/NC Separation}

To determine if an event is CC-like a $k \mathrm{NN}$ method is applied. Firstly a candidate CC-like event must contain a reconstructed track. However, NC events can also have tracks from hadrons. The $k \mathrm{NN}$ method uses a number of event variables 
related to the distinct features of a muon track from a true $\mathrm{CC}$ event. To determine how likely an observed data event is to be CC-like the event is compared to the nearest neighbours of an MC training sample which is spilt into two groups: one set includes $\mathrm{CC}$ events (signal) with reconstructed track(s) and the other is NC events (background) with reconstructed track(s).

Similarly to the $k \mathrm{NN}$ algorithm for CC shower energy estimation, the $k$ nearest neighbours are used to determine the particle identification (PID) metric of the observed event. The distance of a neighbour is determined by the Euclidean metric defined earlier for CC shower estimation in equation 4.2. To give maximum sensitivity in the $\mathrm{CC}$ sample, $k=80$ was chosen for the number of nearest neighbours, and the PID is defined as

$$
\mathrm{PID}=\frac{k_{S}}{k_{S}+k_{B}}=\frac{k_{S}}{k}
$$

where $k_{S}$ and $k_{B}$ are the number of signal events and the number of background events, respectively in the sample of the $k$ nearest neighbours.

Two different PID variables are calculated using the $k$ NN method: roID [135] and jmID [136]. The CC selector is such that for an event to be selected it must satisfy either roID $<0.25$ or $\mathrm{jmID}<0.5$. These PIDs are explained in detail below.

\section{Particle Identification Using roID}

The roID is calculated using four different event variables [135]. The distributions of these variables for both signal and background are shown in figure 5.12. The four variables used are:

1. The number of active planes in a track: This measures the number of scintillator planes with energy deposition belonging to the track and no hadronic shower hits. This is effectively a measure of the extension of the 
track past the end of the hadronic shower, which is correlated to track energy. A muon track will typically extend further than a hadronic track.

2. Mean pulse height of track hits: Defined as the average pulse height of the energy deposition in the track divided by the expected pulse height from a minimum ionising particle. This variable takes advantage of the minimally ionising nature of the muon as it deposits a constant amount of energy in each plane, resulting in a peak in the distribution for $\mathrm{CC}$ events around 1 unit. Background events will have a much broader distribution.

3. Signal fluctuation: This quantifies how much the energy deposition in successive scintillator planes fluctuates. A non-muon track will have larger fluctuations in energy deposition compared to a muon track. The distribution for muon tracks (signal events) on average will tend to have a sharper, more defined peak.

4. Transverse track profile: The transverse track profile measures the isolation of the muon track from the associated hadronic shower. A muon will typically deposit energy in a single scintillator strip, whereas a hadronic shower will be spread over multiple strips within a plane. The transverse track profile is computed by looking at the $50 \%$ of the track scintillator planes furthest from the track vertex. Looking at the planes with track hits, all hits in a 4 strip window around the track hits are considered. The transverse track profile is the ratio of the pulse height in track hits to all hits in the 4-strip window. A CC event will have a narrow, well defined peak compared to background.

These four topological variables are used to calculate a single variable used to discriminate between a CC-like (signal) or NC-like (background) event, which is called the roID. The distribution of this parameter can be seen in figure 5.13. An optimisation was performed similar to that of the $k \mathrm{NN}$ shower energy estimator 

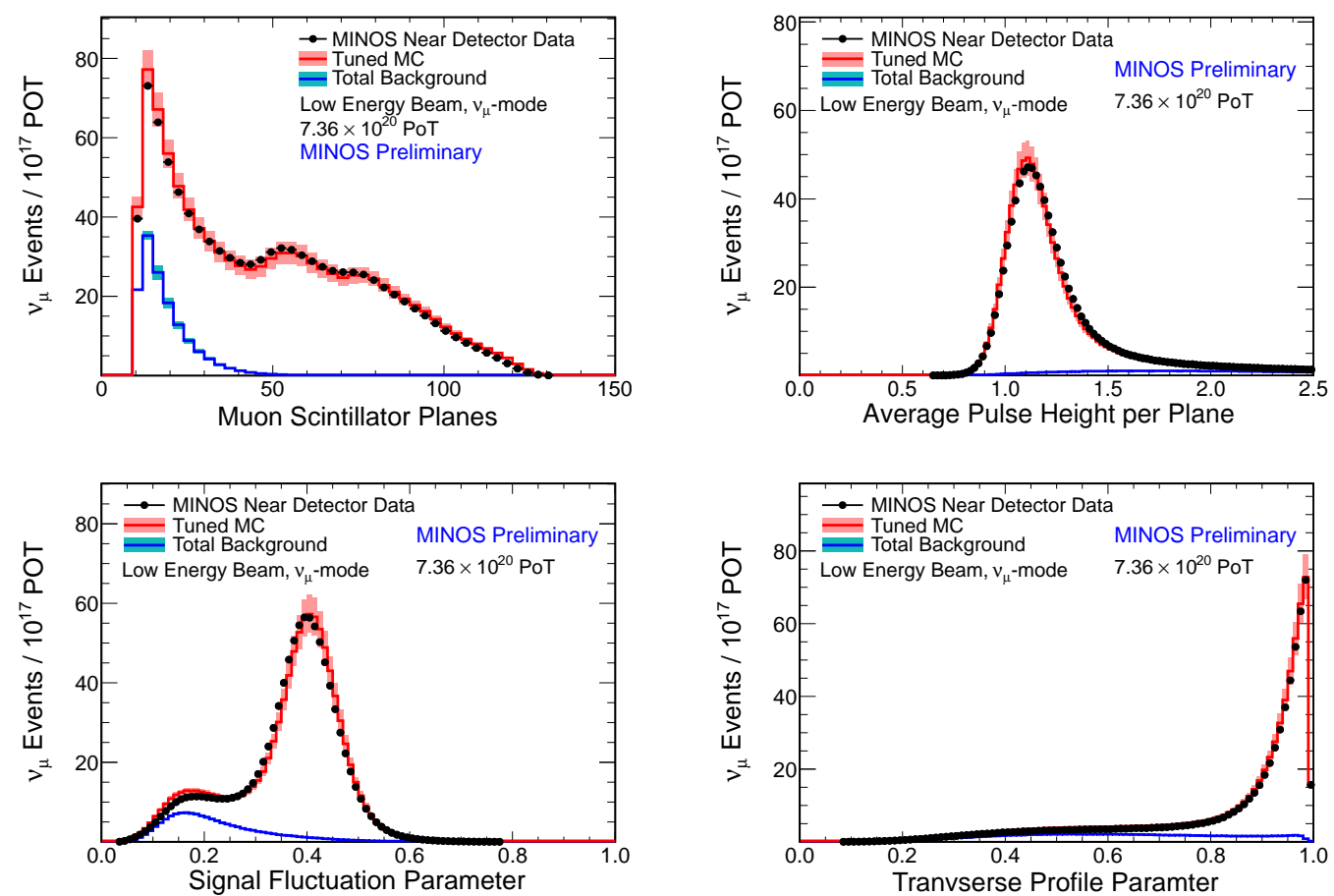

Figure 5.12: Distribution at the Near Detector of the four input variables used to calculate the roID CC/NC separation parameter. Shown is MC (red), data (black), and NC background in the selected MC (blue) with systematic uncertainties on the MC shown by the shaded regions. 


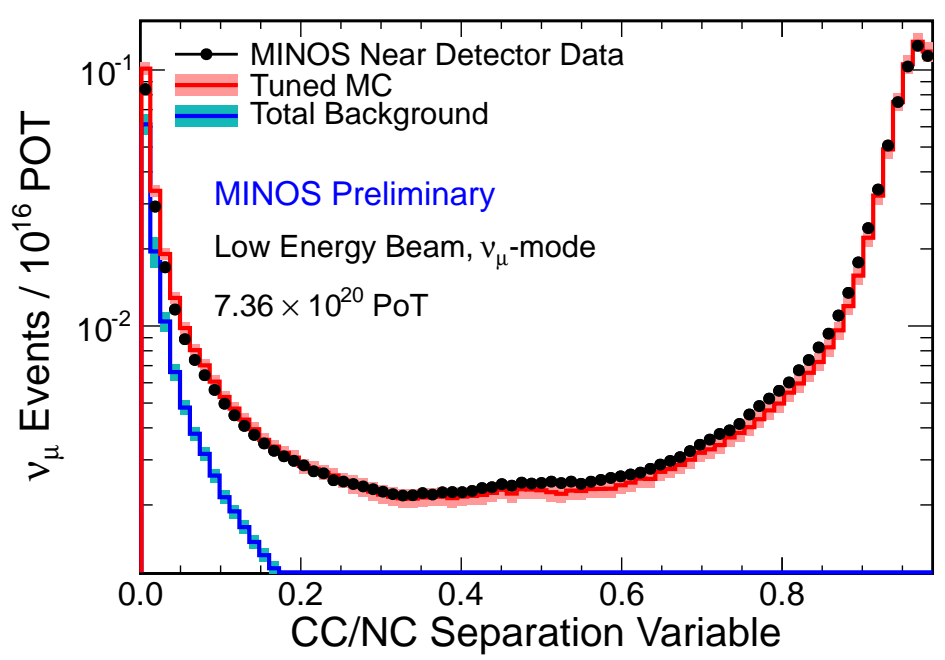

Figure 5.13: The roID distribution at the ND. The red histogram represents the Monte Carlo expectation with systematic uncertainties, and the blue histogram represents the NC background with systematic uncertainties. Black points represent data. The selection criteria removes events with roID $<0.25$.

on the sensitivity on the parameters $\Delta m_{32}^{2}$ and $\theta_{23}$ using the CC sample [135], it was found that the maximum sensitivity was gained by having the selector accept events in the $\mathrm{CC}$ sample if roID $<0.25$. A similar roID is calculated at the MINOS FD.

\section{Particle Identification Using jmID}

The roID variable alone achieves a good $\mathrm{CC} / \mathrm{NC}$ separation over most neutrino energies. However, at low energies, there is scope for improvement where the short muon and hadron tracks become hard to distinguish. At lower energies roID relies less on the "number of scintillator planes" variable and more on the other three. To maximise the sensitivity using the roID PID any events with muon tracks with fewer than 10 scintillator planes are discarded; thus the roID ignores a substantial number of low-energy events with short tracks.

A second PID variable is used called the jmID, which serves to address the low-energy event issue in the roID method and is optimised in the $0-0.5 \mathrm{GeV}$ region. This variable also uses the $k \mathrm{NN}$ method with four topological variables 
shown in figure 5.14 that are defined as follows:

1. The number of active planes in a track: The same definition as that for roID except that events with short tracks are also included.

2. Sum of track-end pulse height: The pulse height at the end of a track for a proton or pion is significantly larger than a muon. Thus by summing up the pulse height towards the end of the track, this quantity can be used to help distinguish a muon track from a non-muon track.

3. Two degrees of scattering: Muon tracks are typically straight compared to hadronic tracks which will have a higher variation plane to plane due to increased scattering. Therefore the scattering is quantified for the $U$ and $V$ planes separately using the Pearson correction coefficient for the track,

$$
\rho=\frac{1}{N} \frac{\sum_{i}^{N} x_{i} z_{i}}{\sigma_{x} \sigma_{z}}
$$

where $x$ and $z$ are the transverse and longitudinal position of the hit $i$ along the track and $\sigma_{x}$ and $\sigma_{z}$ are the widths of the distributions with $N$ being the total number of hits associated with the track. The final scattering variable is defined as

$$
P=\frac{0.01}{1.01-\rho}
$$

The distributions of the scattering variables in the $U$ and $V$ planes can be seen in figure 5.14. Both distributions look identical due to the symmetry in the way $U$ and $V$ planes are read out. On average, the degree of scattering for a non-muon track is smaller than than of a muon track.

The $k \mathrm{NN}$ variable created using the distributions in figure 5.14 can be seen in figure 5.15. Events are removed from the CC sample if they have a value jmID $<0.5$. 

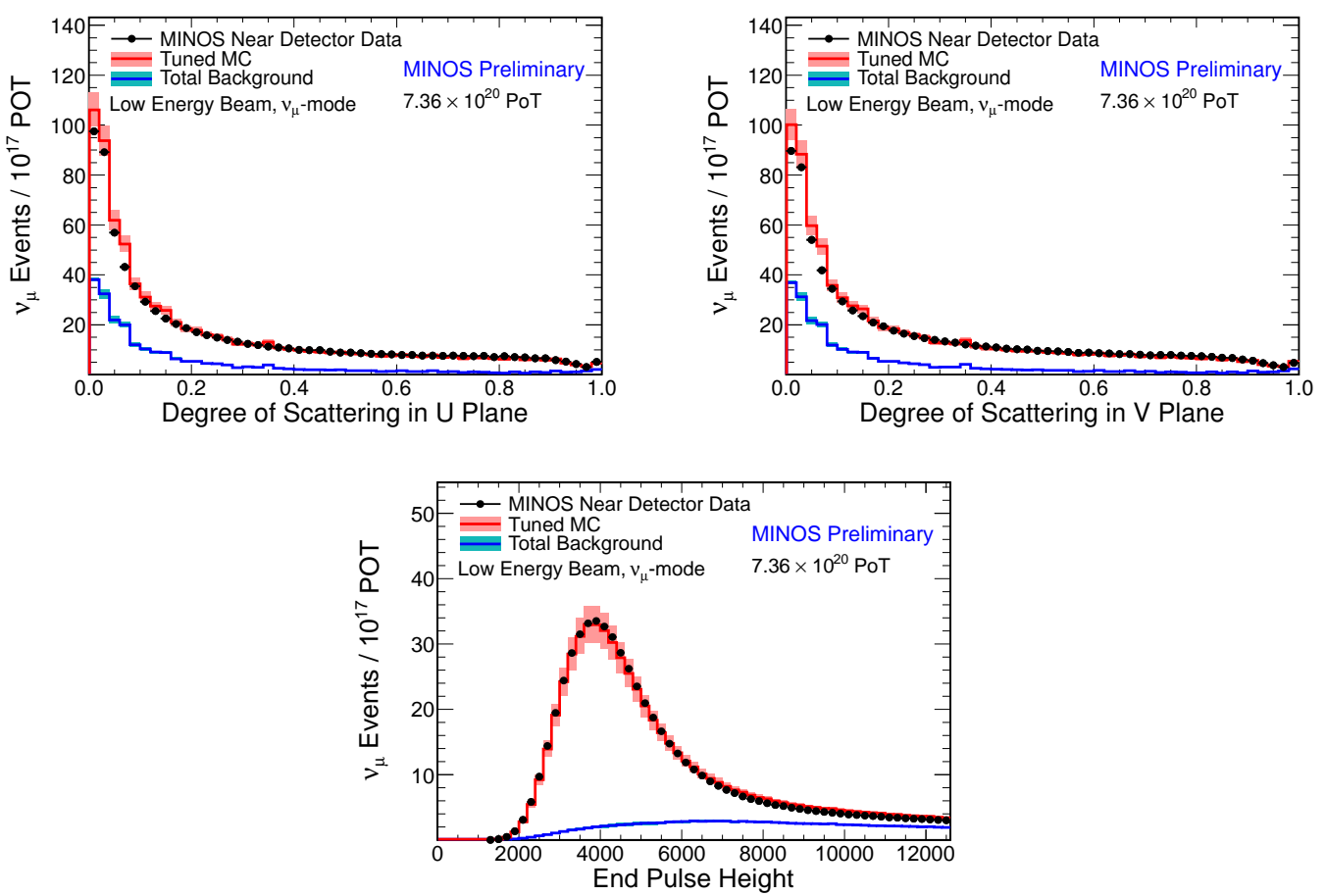

Figure 5.14: Distributions at the Near Detector for the three additional input variables used to calculate the jmID CC/NC separation parameter. Shown is selected MC (red), data (black), and NC background in the selected MC (blue) with systematic uncertainties on the MC shown by the shaded regions. 


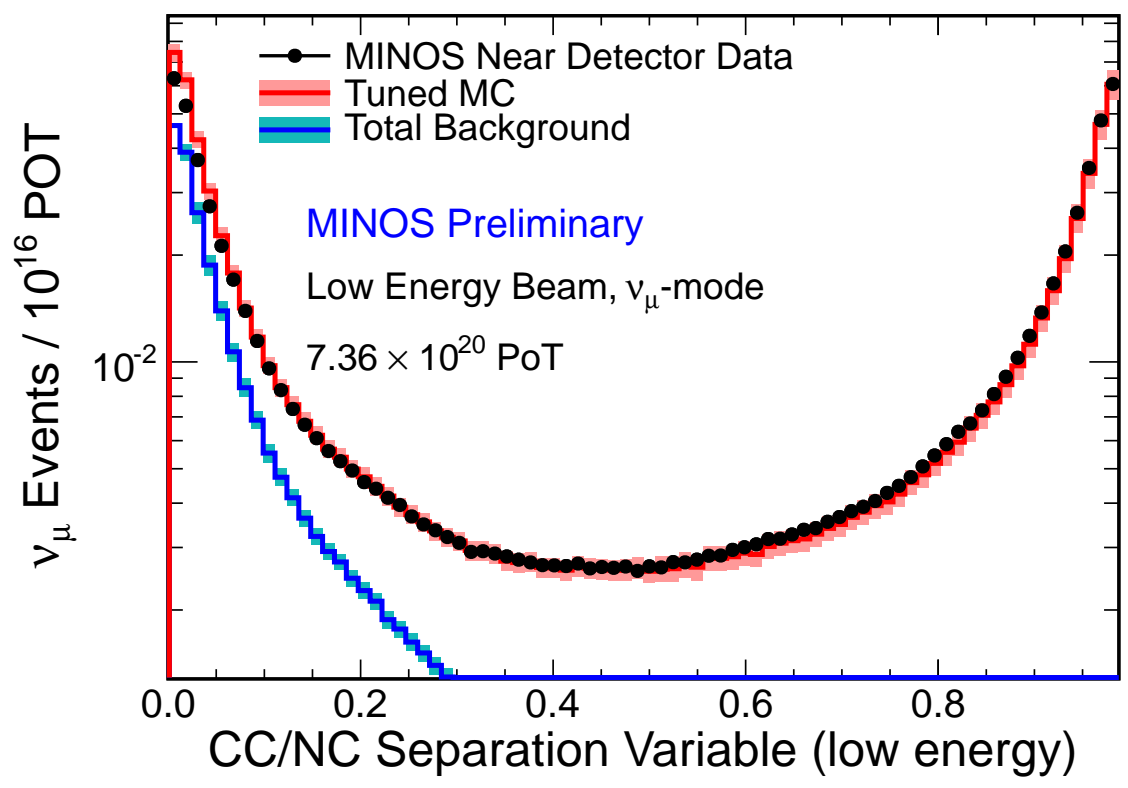

Figure 5.15: The jmID distribution at the MINOS ND. The red histogram represents the Monte Carlo expectation with systematic uncertainties, the blue histogram represents the NC background with systematic uncertainties. Black points represent data. The selection criteria removes events with jmID $<0.5$. The jmID variable is optimised for events between $0-0.5 \mathrm{GeV}$.

\subsection{Selector Performance}

There are two quantities that are used to evaluate a selector's performance. Purity defines how pure a sample is, a metric to determine how many of the selected events are signal events and is defined as

$$
\text { Purity }=\frac{\text { number of selected true signal events }}{\text { total number of selected events }}
$$

Efficiency is used to determine how many of the true signal events are selected from the total dataset, and is defined as

$$
\text { Efficiency }=\frac{\text { number of selected true signal events }}{\text { total number of true signal events before selection }} \text {. }
$$

Figures 5.16 and 5.17 show the purity and efficiency for both MINOS detectors for $\mathrm{CC}$ and $\mathrm{NC}$ selectors respectively. 


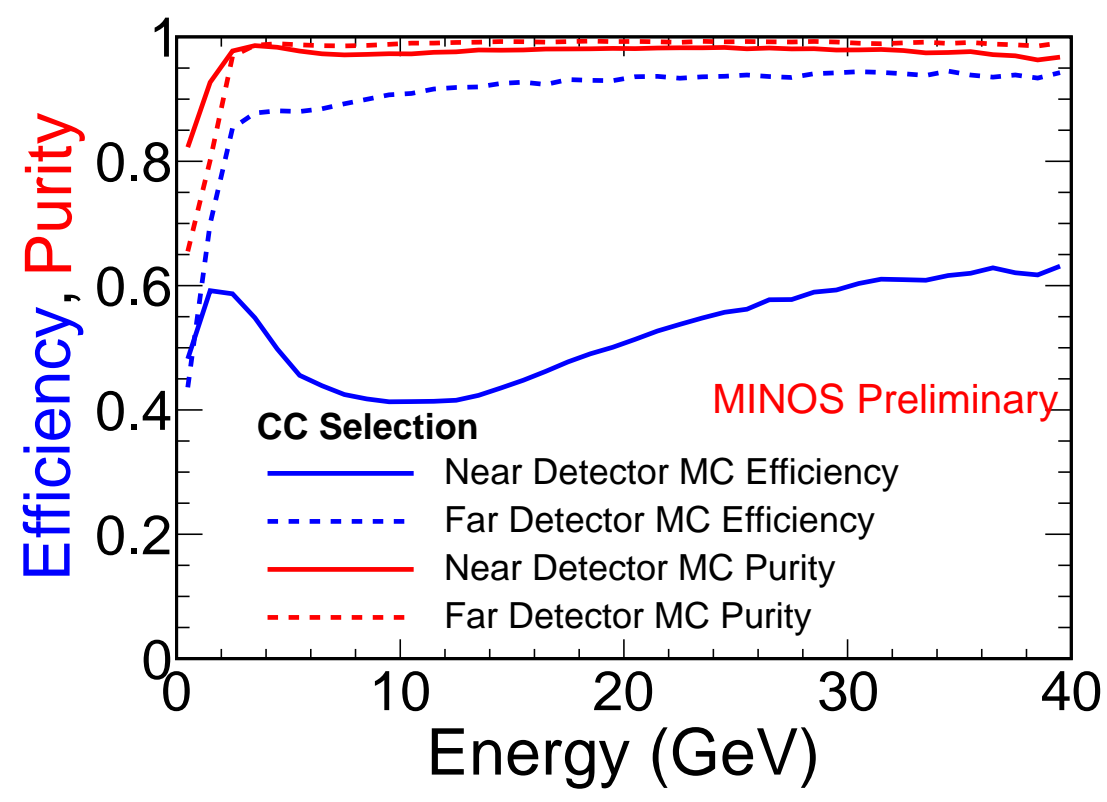

Figure 5.16: Purity and efficiency for the CC selector for the FD and ND as a function of neutrino energy.

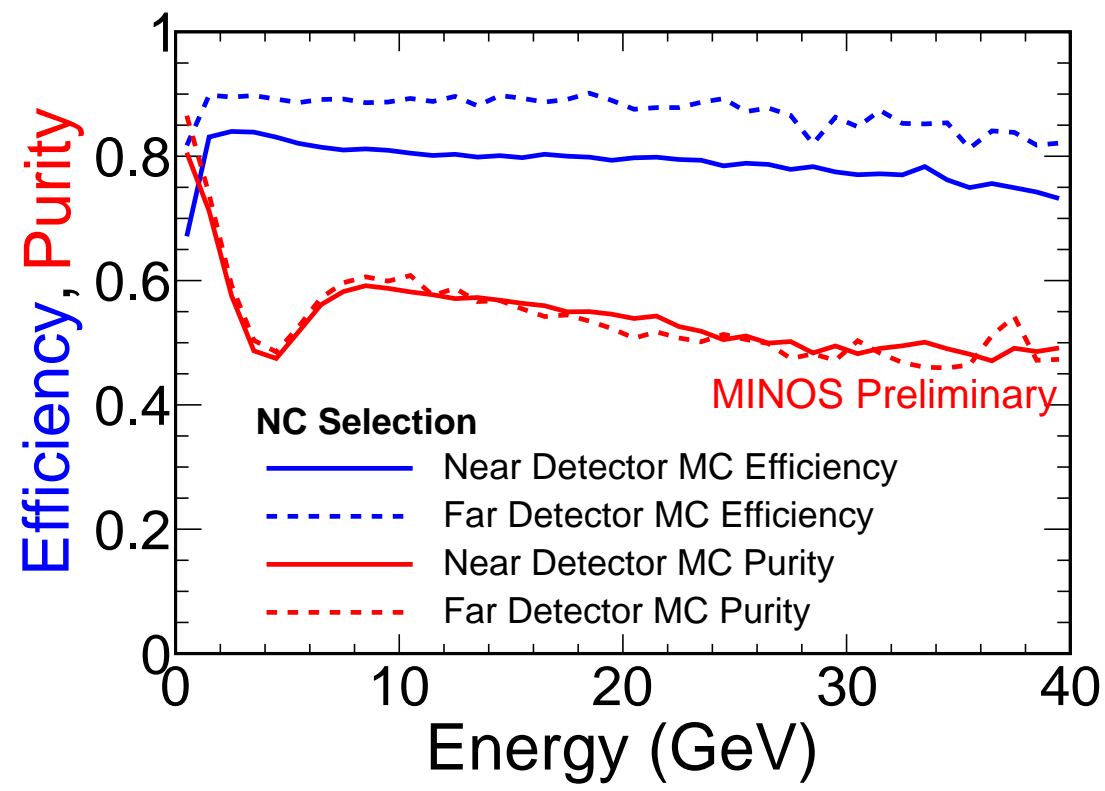

Figure 5.17: Purity and efficiency for the NC selector for the FD and ND as a function of neutrino energy. 


\begin{tabular}{c|c|c} 
Detector, Selector & Efficiency (\%) & Purity (\%) \\
\hline \hline FD, CC & 84.6 & 99.1 \\
ND, CC & 53.9 & 98.7 \\
FD, NC & 87.6 & 61.3 \\
ND, NC & 79.9 & 58.9
\end{tabular}

Table 5.1: Efficiency and purity of charged and neutral-current event selectors in the energy window $0-40 \mathrm{GeV}$.

MINOS was designed for measuring muon-neutrino disappearance using CC interactions, and it is therefore this sample that has the highest purity. The total efficiencies and purities of both the $\mathrm{NC}$ and $\mathrm{CC}$ samples over the $0-40 \mathrm{GeV}$ analysis window are shown in table 5.1. The efficiency of the ND for CC events is low due to the coil-hole cut described earlier. The dip in the efficiency in the ND for $\mathrm{CC}$ events at $5-15 \mathrm{GeV}$ is attributed to the coil-hole cut. Charged current events with final state muons within this energy range typically enter the coil hole region, therefore the coil-hole cut requirement causes this shape for the ND CC event efficiency. Due to the large statistics at the ND the low efficiency has a negligible impact on this analysis. The NC efficiencies at both the FD and ND are low as MINOS struggles to differentiate between $\mathrm{NC}$ and inelastic CC events, which are events with short tracks and with most of the initial energy in the hadronic shower. 


\section{Chapter 6}

\section{Systematic Uncertainties}

This chapter will discuss the evaluation of systematic uncertainties that may affect the ND and FD CC and NC energy spectra used in the sterile neutrino analysis. A breakdown of each systematic uncertainty is explained and quantified in terms of the uncertainty on the ratio of the FD to ND energy spectra which, from now on, will be referred to as the $\mathrm{F} / \mathrm{N}$ ratio. The last section will describe the procedure used to generate covariance matrices such that the uncertainties can be combined and then incorporated into the analysis described in chapter 7 .

\subsection{Introduction}

Physics simulation packages do not perfectly model the physics of the world around us; with all models, there are uncertainties that arise that, if not properly assessed and accounted for, have the potential to affect the measured oscillation parameters during a fitting procedure.

Systematic uncertainties allow one to quantify an error in the modelling of an underlying physics process that should be incorporated into a fit to prevent an analysis from over-fitting features in the data that are not inherently fundamental but arise due to a shift in the energy of selected events, an over(under)estimate of the number of selected events, or a mis-estimate of the number of predicted 
background events. A description of the systematic uncertainties evaluated that have an effect on the $\mathrm{F} / \mathrm{N}$ ratio used in the analysis presented in this thesis is given below.

\subsection{Detector-Related Systematic Uncertainties for $\mathrm{CC}$ and $\mathrm{NC}$ samples}

The sources of uncertainty can be broken down into the main components: relative normalisation, acceptance and selection efficiency in the ND, track/shower absolute energy scale, poorly-reconstructed events, neutrino cross sections, backgrounds in both the $\mathrm{CC}$ and $\mathrm{NC}$ samples, hadron production, and beam focusing.

\subsubsection{Relative Normalisation}

The relative $\mathrm{F} / \mathrm{N}$ normalisation uncertainty is one of the dominant systematic uncertainties for both the $\mathrm{CC}$ and NC samples. This uncertainty can be broken down into its main components: steel thickness, scintillator thickness, FD live time, differences in reconstruction efficiency between the FD and ND and ND selection bias [137]. The dominant component arises from the differences in reconstruction efficiencies that is unaccounted for by MC.

The relative normalisation uncertainty for the NC sample is evaluated by selecting $\mathrm{CC}$ events that have successfully passed through the reconstruction software, then removing the hits associated with the muon track to mimic NC events. These muon-removed charged-current events are passed through the reconstruction chain again, and may or may not be successfully reconstructed. This allows for a data-driven calculation of the reconstruction efficiencies to be performed for both the ND and FD. The resulting uncertainty is $2 \%$. This is then combined in quadrature with the uncertainties of the steel and scintillator thickness, ND selection bias and uncertainty in the determination of the FD live time resulting 
in a total uncertainty of $2.2 \%$ across all energies [137].

For the CC sample sets of data and MC events in both detectors were scanned by a team of people to identify events that failed the reconstruction process and events that moved in or out of the fiducial volume. The total normalisation uncertainty for the CC sample comes to $1.54 \%$ across all energies [137]. The resulting uncertainty on the $\mathrm{CC}$ and $\mathrm{NC} \mathrm{F} / \mathrm{N}$ ratios is shown in figure 6.1.
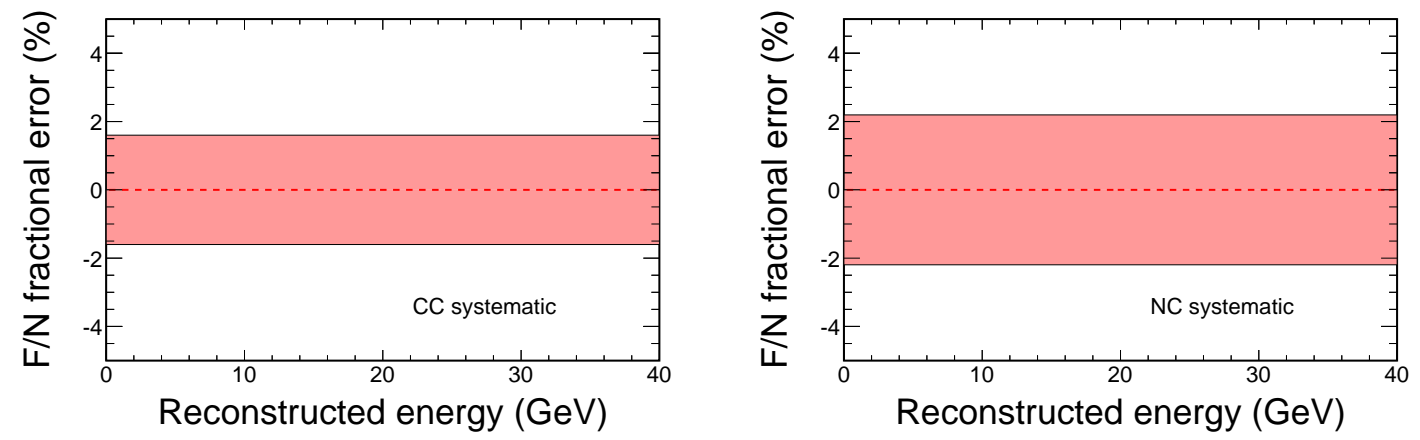

Figure 6.1: CC (left) and NC (right) fractional errors from the relative normalisation uncertainty on the $\mathrm{F} / \mathrm{N}$ ratios.

\subsubsection{Acceptance and Selection Efficiency in the ND}

This systematic accounts for the uncertainty on the acceptance and selection efficiency in the ND. This uncertainty was evaluated by varying event selection requirements in data and $\mathrm{MC}$ for both the $\mathrm{CC}$ and $\mathrm{NC}$ samples in order to probe weaknesses in the simulation. In each case, the variation in the ND data-to-MC spectral ratio seen when varying the cuts is taken as the systematic on the $\mathrm{F} / \mathrm{N}$ ratio.

\section{NC Sample}

A previous study of spatial uniformities associated with event reconstruction at the ND can be found in reference [138]. This study varied the selection criteria for data and MC for different quadrants, radial annuli and $z$-segments of the ND 
fiducial volume. Although this study shows any disagreement to be small, two spatial variation studies were performed to quantify this systematic for the NC selection sample.

- Difference in the ND NC energy spectrum between events originating in the left and right halve of the fiducial volume of the ND.

- The change in the NC energy spectrum when tightening the fiducial $z$ cut at the ND from $4.7368 \mathrm{~m}$ to $2.5 \mathrm{~m}$.

\section{Sample}

Similarly to the NC sample the ND event selection cuts for CC events are varied for both data and MC and the differences in the ND data to MC spectral ratio were taken to be the systematic error on the $\mathrm{F} / \mathrm{N}$ ratios.

- Differences in the ND CC energy spectrum between events originating in the left and right halves of the fiducial volume of the ND.

- Tightening the fiducial $z$ cut at the ND from $4.077 \mathrm{~m}$ to $2.5 \mathrm{~m}$.

- Varying the fiducial radius from $80 \mathrm{~cm}$ to $60 \mathrm{~cm}$.

- Not implementing the coil hole cut.

- Removing all events with a track ending within 10 planes of the start of the spectrometer in the ND.

- Allowing events with a track exiting the side of the calorimeter, which are normally removed.

- Removing all events with a track ending within 10 planes of the end of the ND.

The uncertainty on the $\mathrm{F} / \mathrm{N}$ ratio from all these sources of uncertainty are summed in quadrature to give the total uncertainty which is shown in figure 6.2. 

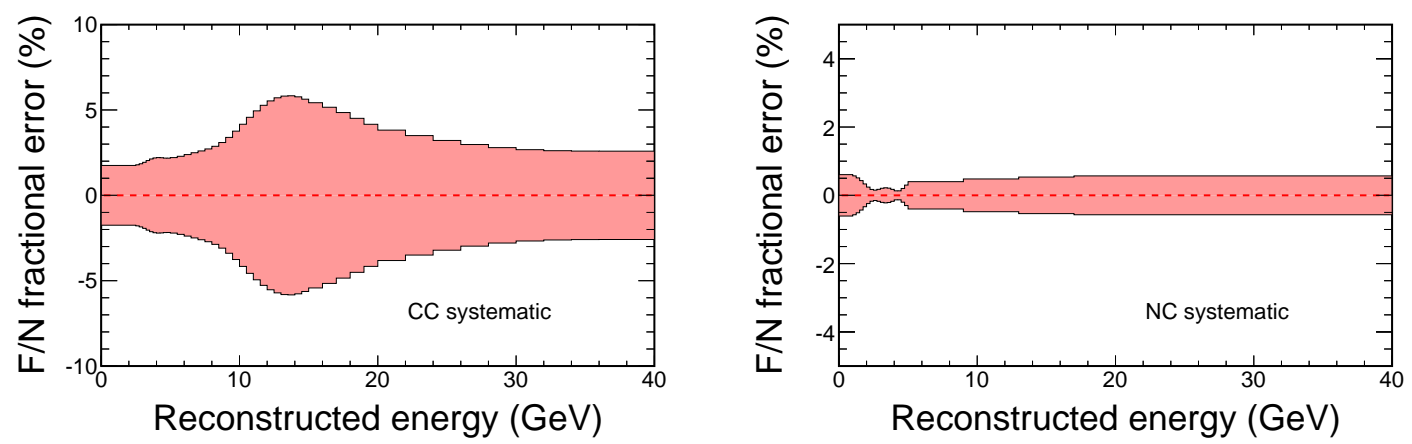

Figure 6.2: CC (left) and $\mathrm{NC}$ (right) fractional errors from the combined acceptance uncertainties on the $\mathrm{F} / \mathrm{N}$ ratios.

\subsubsection{Track and Shower Energy Scale}

\section{Muon track energies}

The muon track energy can be measured to within $2 \%$ from range; this uncertainty is calculated from the uncertainty in detector density and geometry as well as uncertainties on particle propagation. The muon track energy measured via curvature has an uncertainty of $3 \%$, which is evaluated by comparing the differences in the range and curvature measurements of stopping muon tracks [135].

\section{Shower energies}

The calibration chain in chapter 4 and $k \mathrm{NN}$ shower energy estimator described in section 4.4.1 are used to convert detector response to measured hadronic shower energies. This calibration chain has two different hadronic energy uncertainties to consider: the relative mis-modelling between both detectors and the absolute mis-modelling.

The relative mis-modelling comes from the calibration uncertainty of the two detectors; for the ND the uncertainty is $1.9 \%$ and for the FD is $1.1 \%$.

The absolute shower uncertainty comes from a number of sources: the uncertainty on energy deposition of hadrons in CalDet which is $5 \%$; the beam energy uncertainty used at CalDet which is $2 \%$; the uncertainty in the stopping muon 
calibration at CalDet which is $1.4 \%$; and the cosmic MEU numbers which are consistently lower for cosmic muons in both the FD and ND than for muons from the neutrino beam giving a $0.9 \%$ uncertainty. Combined in quadrature this gives a systematic uncertainty of $5.7 \%$ at all shower energies [139].

The calibration chain relies on the modelling of stopping muons to obtain the hadronic energy scale. Any mis-modelling in the simulation used to model these stopping muons in the detector will cause additional disagreement between data and simulation. This is explored in detail in references [140] and [131] by varying the cross section, branching ratios and formation lengths used in simulating events and observing the effect it has on the corresponding hadronic shower energy. The study estimates an energy-dependent uncertainty on measured shower energies. For showers energies $<0.5 \mathrm{GeV}$ an uncertainty of $8.32 \%$ is given, and this decreases to $\sim 3.5 \%$ for energies above $6 \mathrm{GeV}$.
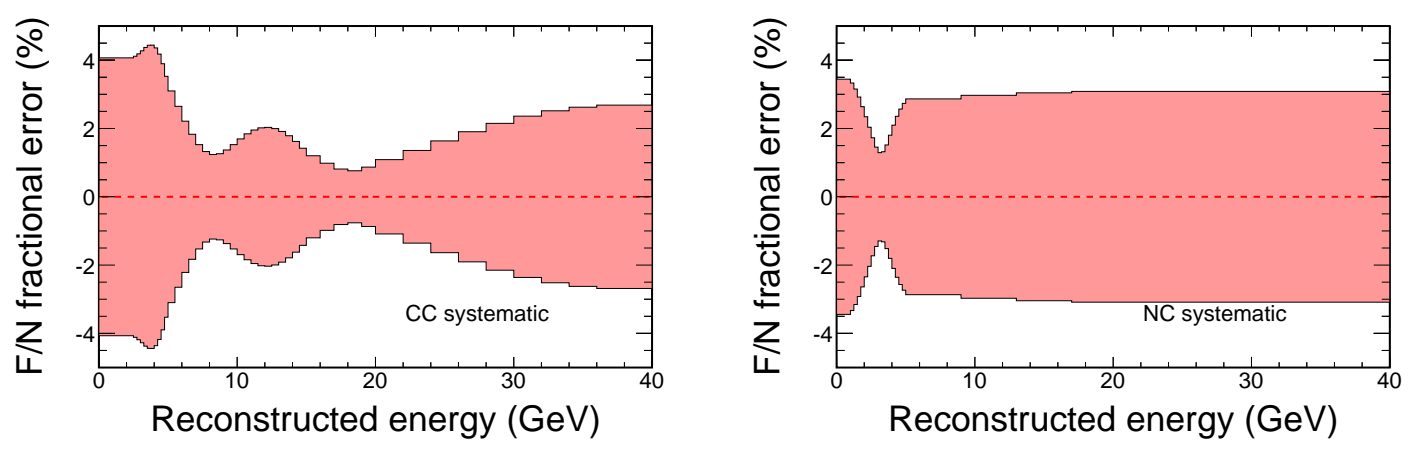

Figure 6.3: CC (left) and NC (right) fractional errors from the combined energy uncertainties on the $\mathrm{F} / \mathrm{N}$ ratios.

The uncertainties from the calibration process (a constant $5.7 \%$ across all energies) and hadronic modelling are believed to be uncorrelated and are combined in quadrature to give a total energy-dependent uncertainty on calorimetric energy measurement that can be modelled by an exponential parameterisation as a function of shower energy $E_{\mathrm{shw}}$ : 


$$
\sigma_{\mathrm{shw}}=6.6 \%+3.5 \% \times \exp \left[-\frac{E_{\mathrm{reco}}}{1.44 \mathrm{GeV}}\right]
$$

where the correlations between energy bins is taken to be $100 \%$. In section 4.4 .2 it was shown that the $k \mathrm{NN}$ shower energy estimator, used for $\mathrm{CC}$ events, is more robust against systematic uncertainties than the calorimetric method. However, for historical reasons, this slightly more conservative calorimetric uncertainty is used in this analysis.

By varying both the FD and ND spectra by their $\pm 1 \sigma$ errors. It is the differences in the $\mathrm{F} / \mathrm{N}$ ratio for $\mathrm{MC}$ by shifting the $\mathrm{F} / \mathrm{N}$ ratio by the known systematic error that are used to calculate the $\mathrm{F} / \mathrm{N}$ systematic uncertainty. The uncertainties on the $\mathrm{CC}$ and $\mathrm{NC} \mathrm{F} / \mathrm{N}$ ratios arising from the track and shower energy uncertainties are shown in figure 6.3.

\subsubsection{Poorly-Reconstructed Events}

This uncertainty affects only the NC sample. The two variables used to remove the poorly-reconstructed events are not perfectly modelled by $\mathrm{MC}$ with the uncertainty coming from the combination of the modelling of the poorly-reconstructed component and on the cut position of the preselection variables. The uncertainty on the procedure used to remove poorly-reconstructed events is used to evaluate the resulting systematic error on the $\mathrm{F} / \mathrm{N}$ ratio.

The general idea is to change the position of the cuts on the poorly-reconstructed event-cleaning variables in MC such that the rejected events from these distributions agree with the fraction rejected by the data at the ND (where the data is fixed to always use the nominal cut values). Since poorly-reconstructed events have energies predominately below $2 \mathrm{GeV}$, three reconstructed energy bins were considered for this study: $E_{\text {reco }}<5 \mathrm{GeV}, 1 \mathrm{GeV}<E_{\text {reco }}<5 \mathrm{GeV}$, and $E_{\text {reco }}>1 \mathrm{GeV}$. To fully assess the correlation between the two contributions to 
the systematic error the uncertainty is evaluated by a two step procedure [141]:

1. For each energy bin, the poorly-reconstructed component is allowed to be freely scaled such that the total MC is in good agreement in the distributions of the two preselection variables.

2. After a suitable scaling is chosen the preselection cut values are altered in MC such that the same number of events are rejected in both data and MC.

The reconstructed energy distribution at the ND for $\mathrm{MC}$ with these altered preselection cuts is compared to the nominal MC energy distribution. It is these differences that are taken as the systematic error on the $\mathrm{NC} \mathrm{F} / \mathrm{N}$ ratio as shown in figure 6.4.

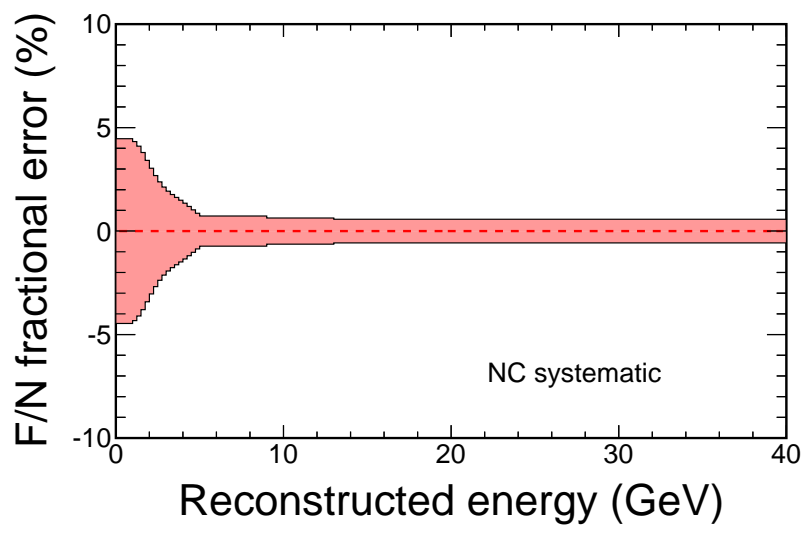

Figure 6.4: Error on the neutral current Far-over-Near ratio arising from uncertainties on the number of poorly-reconstructed events in the sample.

\subsubsection{Neutrino Cross-Sections}

Neutrino cross sections are used in MINOS detector simulations provided by the NEUGEN event generator [116] using the MODBYRS-4 cross section model [120, $118,119]$. The process used to evaluate the energy-dependent cross-section uncertainties involves changing the NEUGEN parameters [142].

For energies up to $\sim 1 \mathrm{GeV}$ the dominant neutrino interactions are quasi-elastic scattering and resonance production. Each of these is modelled in NEUGEN 
using a single parameter approach, the axial masses, $M_{\mathrm{A}}^{\mathrm{QE}}$ and $M_{\mathrm{A}}^{\mathrm{Res}}$ respectively. In the simulation the values of both axial masses are set to $1 \mathrm{GeV}$ which is consistent with the global average $M_{\mathrm{A}}=1.03 \pm 0.02 \mathrm{GeV}$ [143]. The best information on these parameters comes predominately from neutrino-nucleus data in deuterium-filled bubble chambers. An uncertainty of $15 \%$ is associated with these parameters in order to cover any disagreements between data and simulation [144, 142]. During this analysis MINOS released a measurement of the axial mass by studying charged-current $\nu_{\mu}$ interactions on iron giving a value of $M_{\mathrm{A}}^{\mathrm{QE}}=1.23_{-0.09}^{+0.13}(\text { fit. })_{-0.15}^{+0.12}$ (syst.) [145]. This measured value is quite higher than previous measurements, however for this analysis the neutrino cross section systematic is not one of the dominant sources of uncertainty, with the cross section uncertainty not being strongly dependent the axial mass values. It was considered not worth while re-generating the simulation with the updated MINOS value on the axial mass as the effect would have negligible impact on this analysis.

The resonance to DIS transition region $(1-10 \mathrm{GeV})$ is modelled by a set of parameters $r_{i j k}, i=1,2$ referring to $\mathrm{CC}$ and NC interactions, $j=1,2,3,4$ denoting the type of interaction between either $\nu_{\mu}$ or $\bar{\nu}_{\mu}$ and either a proton or a neutrino $(1 \equiv \nu p, 2 \equiv \nu n, 3 \equiv \bar{\nu} p, 4 \equiv \bar{\nu} n)$, and $k$ denoting the multiplicity of the final state (KNO multiplicity). Fits to data indicate uncertainties of \pm 0.1 on $r_{i j 2}$ and \pm 0.2 on $r_{i j 2}[142]$.

The $10-30 \mathrm{GeV}$ region is dominated by DIS neutrino interactions. Fits to data combined with global fits suggests a 3.5\% uncertainty on the total neutrino charged current cross section.

The anti-neutrino cross sections are determined by the same cross section model that provides the neutrino cross sections and are governed by many of the same parameters. The anti-neutrino to neutrino cross section ratio is then the remaining source of uncertainty. This uncertainty is treated by an additional set of uncertainties applied to anti-neutrino cross sections that are motivated by their 
deviations from the much more well known neutrino cross sections. Therefore an additional uncertainty of $8 \%$ is used for the total $\bar{\nu}_{\mu}$ quasi-elastic and resonance cross sections along with a $4 \%$ uncertainty on the total charged-current antineutrino cross section. Fitting world data in the $1-10 \mathrm{GeV}$ region gives errors on the resonance to DIS region parameters $r_{i 23}$ and $r_{i 42}$ of \pm 0.2 [142].

The total systematic error on the $\mathrm{F} / \mathrm{N}$ ratio due to varying both the $\mathrm{FD}$ and ND spectra by their $\pm 1 \sigma$ errors for neutrino cross-sections for both the CC and NC samples can be seen in figure 6.5.
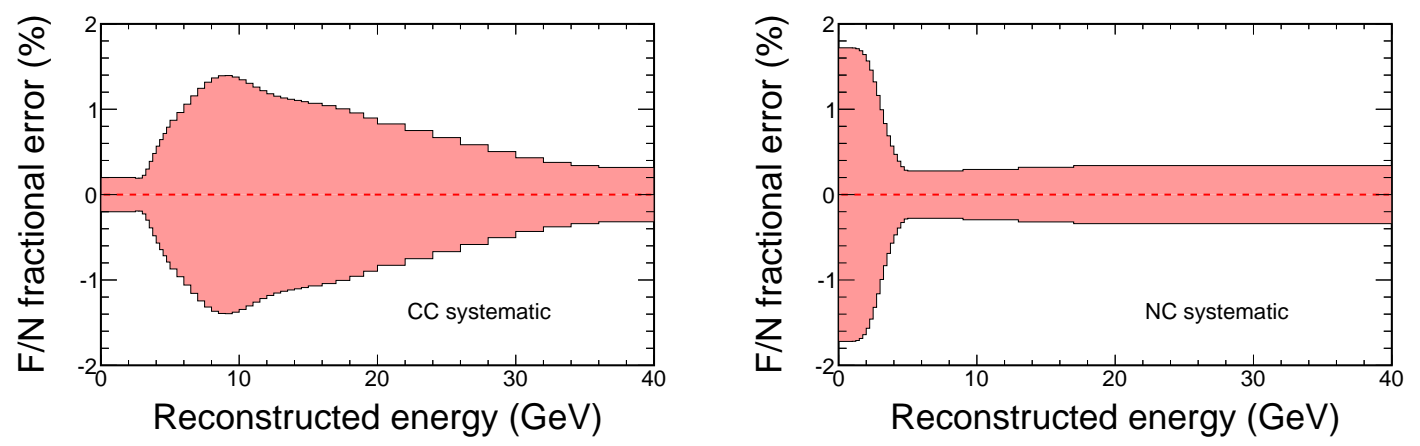

Figure 6.5: CC (left) and NC (right) fractional errors from the combined cross section uncertainties on the $\mathrm{F} / \mathrm{N}$ ratios.

\subsection{6 $\mathrm{CC}$ and NC Backgrounds}

\section{CC sample}

Two methods are used to quantify the uncertainty on the background NC events within the CC sample. The first method is to remove the muon track from wellreconstructed $\mathrm{CC}-\nu_{\mu}$ events to mimic $\mathrm{NC}$ events in both data and $\mathrm{MC}$. Once processed by the reconstruction chain the rate of accidentally reconstructing a $\mathrm{CC}$ event from data or Monte Carlo is evaluated for both data and MC and compared. The uncertainty from this method was found to be $15 \%$. The second independent method was to use the PID distributions for both data and MC (see figure 5.13 as an exmaple). The NC component of the CC sample was freely 
scaled to allow a good agreement with data with a scaling factor of $11 \%$ found to give the best agreement. Combining both uncertainties in quadrature gives a total uncertainty on the amount of background in the CC sample to be $20 \%$ [146].

\section{NC sample}

The uncertainty on the number of CC background events in the NC sample is evaluated using a data-driven method that makes a direct measurement of the CC contamination in the NC-like spectrum for different energy configurations: low energy, medium energy, horn off, and high energy configurations [147]. A detailed description of the procedure can be found in reference [148] which results in a systematic uncertainty of $15 \%$ on the charged current background within the NC sample.

The systematic uncertainty for the backgrounds in the CC and NC samples on the $\mathrm{F} / \mathrm{N}$ ratios is shown in figure 6.6.
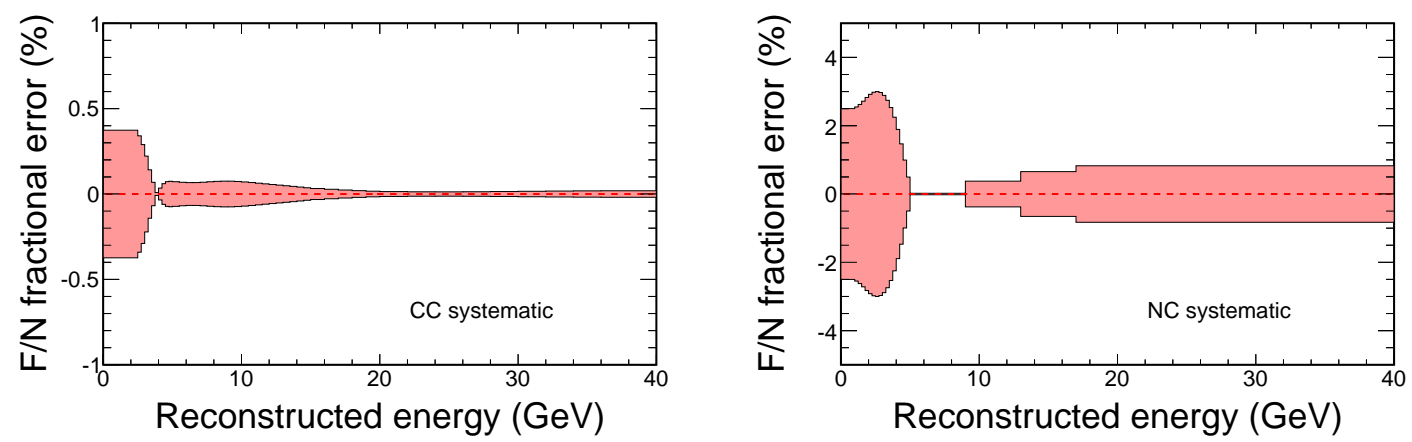

Figure 6.6: CC (left) and NC (right) fractional errors from the background uncertainties on the $\mathrm{F} / \mathrm{N}$ ratios.

\subsubsection{The FD Cleaning and Cosmic Uncertainties}

This systematic comes from the removal of background events from the FD which may contaminate the NC sample. The two main sources of background in the FD that could impact the purity of the NC sample were evaluated in detail in 
reference [149] and discussed in chapter 5. The uncertainties are listed below:

- FD noise mis-reconstructed as a low-energy NC event and light pulses from the light injection system entering the data stream that could be reconstructed as NC events. The uncertainty associated with the removal of these effects is $4.9 \%$ for energies below $0.5 \mathrm{GeV}$ and less than $1 \%$ for events with energies above $0.5 \mathrm{GeV}$.

- Cosmic-muon events that enter the FD at steep angles, thus being reconstructed as a shower. This is a sub-dominant effect compared to FD noise events. The uncertainty associated with the cuts used for the removal of these cosmic events in the FD can be up to $2.7 \%$ depending on the event energy.

The FD cleaning and cosmic uncertainties on the $\mathrm{F} / \mathrm{N}$ ratio are combined in quadrature and shown in figure 6.7.

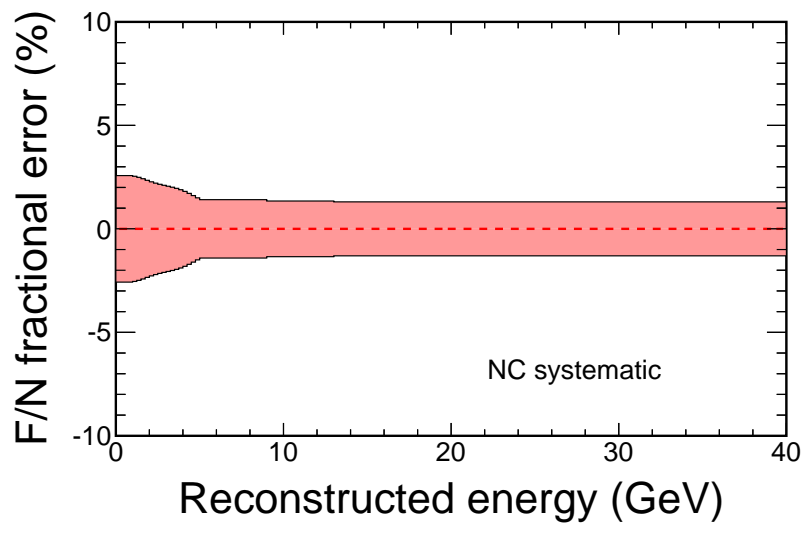

Figure 6.7: Error on the neutral current Far-over-Near ratio arising from uncertainties in the removal of noise, light injection, and cosmic muons.

\subsection{Creation of the Covariance Matrices}

The sterile neutrino analysis presented in chapter 7 fits the $\mathrm{F} / \mathrm{N}$ ratios directly. Therefore in this section it has been the fractional error on the $\mathrm{F} / \mathrm{N}$ ratio for both 
the CC and NC samples that has been calculated.

The sterile neutrino fit incorporates systematic uncertainties using covariance matrices. A covariance matrix, $V$, contains the systematic error on each bin of reconstructed neutrino energy in the $\mathrm{F} / \mathrm{N}$ ratio, plus any correlations between each pair of bins $i$ and $j, \rho_{i j}$. The matrix is expressed as $V(i, j)=\sigma_{i} \sigma_{j} \rho_{i j}$. There is a separate matrix $V$ for each of the $\mathrm{NC}$ and $\mathrm{CC}$ samples.

To produce a covariance matrix from the $\pm 1 \sigma$ error, random numbers are drawn from a Gaussian distribution with unit width. For these randomly sampled numbers, $\xi$, an interpolation is performed on the fractional error bands expressed by $[150]$

$$
e_{i}=\left[\frac{1}{2} \xi(\xi-1) \times \operatorname{down}_{i}\right]+\left[\frac{1}{2} \xi(\xi+1) \times \operatorname{up}_{i}\right]
$$

where $e_{i}$ is the interpolated error for the $i^{\text {th }}$ bin, down $i$ is the $-1 \sigma$ fractional error on the $\mathrm{F} / \mathrm{N}$ ratios in the $i^{\text {th }}$ bin and $\mathrm{up}_{i}$ is the $+1 \sigma$ fractional error on the $\mathrm{F} / \mathrm{N}$ ratio in the $i^{\text {th }}$ bin. If the randomly sampled number, $\xi$, is greater than one then a linear interpolation is performed on the fractional error band.

This process is repeated $N$ times such that a covariance can be computed for all bins of energy, given by

$$
V(i, j)=\frac{1}{N} \sum_{k=1}^{N} e_{k i} e_{k j},
$$

where $k$ represents the $k^{\text {th }}$ drawing of the random number.

\subsection{Hadron Production}

Any analysis using neutrinos from the NuMI beam requires an accurate estimate of the uncertainty on the expected neutrino flux from the NuMI beam. This uncertainty is especially significant for the MINOS sterile neutrino analyses as it 
is sensitive to fluctuations in the high-energy tail, a region known to be modelled poorly in terms of predicting hadron yields [115].

The uncertainty in hadron production was evaluated by studying the differences from the published measurements of the invariant cross-sections for production of $\pi^{ \pm}$and $K^{ \pm}$in proton-carbon collisions at $120 \mathrm{GeV}$ from the NA49 experiment [151], and a simulation of the NA49 target from the same package used to generate the flux predictions at MINOS, FLUKA [112].

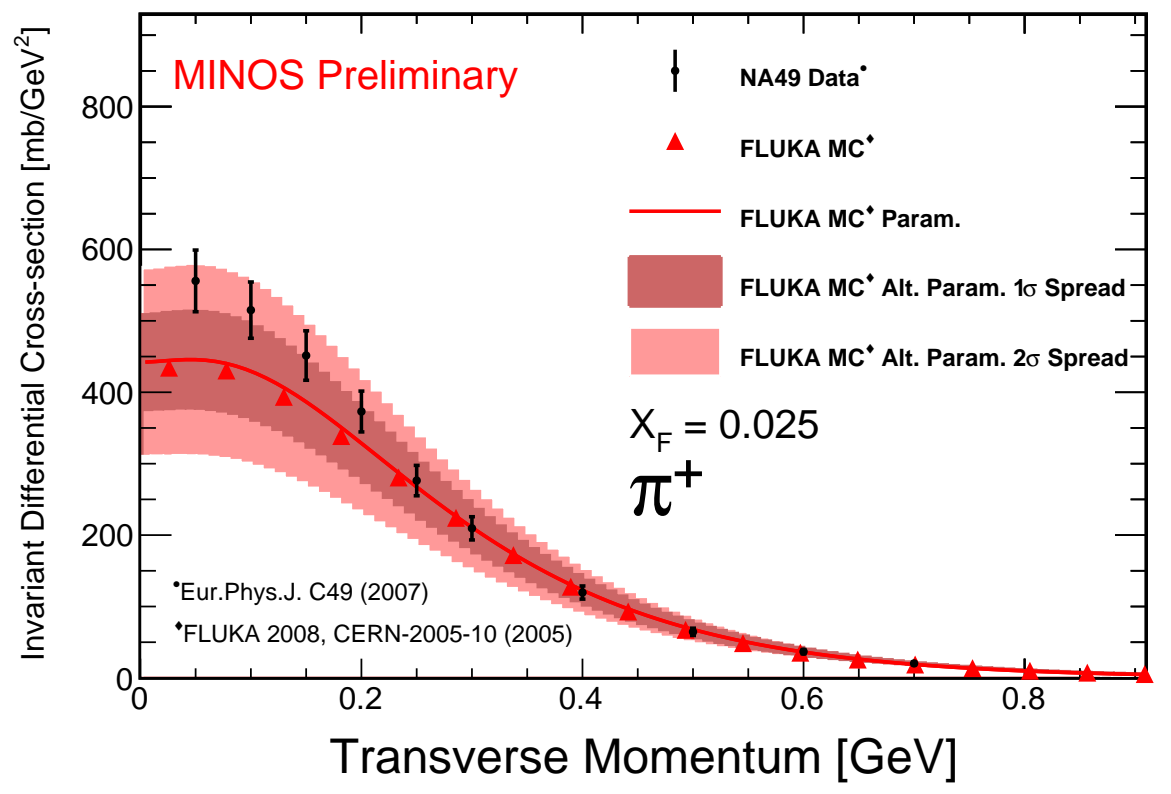

Figure 6.8: Invariant differential cross section for $\pi^{+}$production at $x_{f}=0.025$ as a function of transverse momentum $\left(p_{T}\right)$ produced in $\mathrm{p}+\mathrm{C}$ collisions at $158 \mathrm{GeV} / \mathrm{c}$ beam momentum. Data is shown in solid black, $\mathrm{MC}$ as solid red triangles, the parameterisation of the $\mathrm{MC}$ as a light red line, and example alternative $\mathrm{MC}$ parameterisations are shown as a darker red band for 1 and 2 sigma.

Firstly, an invariant cross-section for a particular hadron type as a function of $p_{t}$ was calculated in a particular bin of $x_{f}$, using the simulation package FLUKA. This was fit as a function of $p_{t}$, as illustrated in figure 6.8, using an empirical parameterisation of inclusive invariant cross sections for secondary particle production (from measured p-Be interactions) known as the BMPT parameterisation [152]. From this fit the best-fit parameters, errors and bin-to-bin correlations were extracted and stored. The fit parameters were then varied within their er- 
rors to create alternative parameterisations, resulting in an error band around the nominal best fit. This was repeated over a range of bins of $x_{f}$.

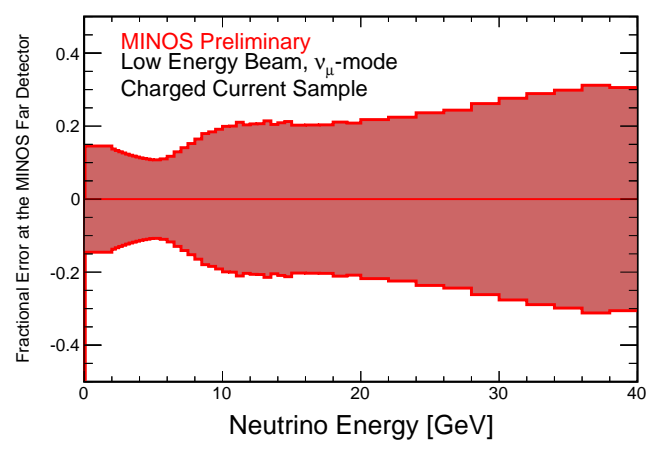

(a) FD CC hadron production error band.

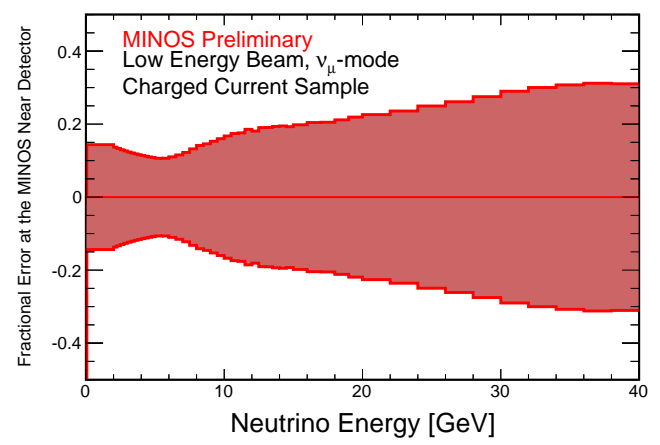

(b) ND CC hadron production error band.

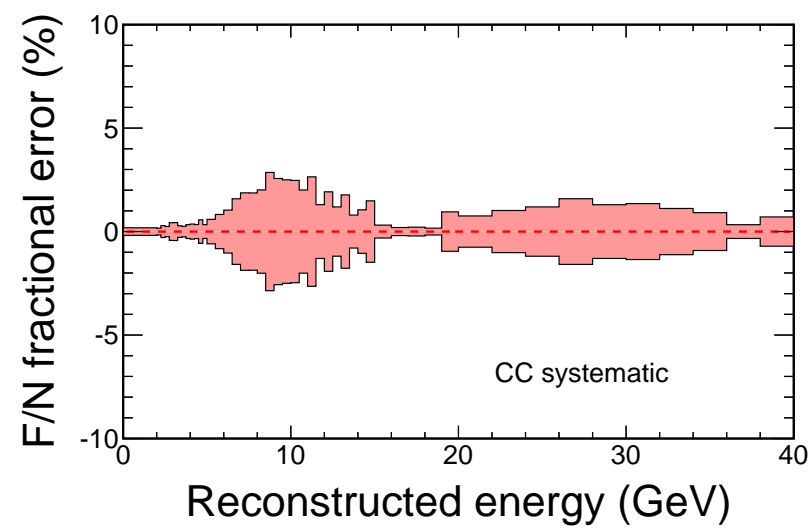

(c) F/N CC hadron production error band

Figure 6.9: The hadron production uncertainty error band as a function of reconstructed neutrino energy for the FD energy spectrum (a), ND energy spectrum (b) and the $\mathrm{F} / \mathrm{N}$ ratio (c) for the $\mathrm{CC}$ sample. The $\mathrm{F} / \mathrm{N}$ error band is the uncertainty used in the sterile neutrino analysis.

This collective set of parameterisations was then compared to the NA49 [151] data in each $x_{f}$ bin. The errors extracted in the previous paragraph from the original fit were scaled up such that the range of parameterisations covered any discrepancy between the data and MC at roughly $68 \%$ C.L. That is, the difference between the data and MC should be smaller than the difference between the best fit and $32 \%$ of the parameterisations. This coverage corresponds to $1 \sigma$ error bands on the invariant cross section for production of $\pi^{ \pm}$and $K^{ \pm}$. An example of such an error band for one particular bin of $x_{f}$ as a function of $p_{T}$ for positive pions is 
shown in figure 6.8 .

The sterile neutrino analysis in this thesis performs the fit on the $\mathrm{F} / \mathrm{N}$ ratio in terms of reconstructed neutrino energy. In order to convert the hadron production uncertainty to an uncertainty on the $\mathrm{F} / \mathrm{N}$ ratio, the assumption is made that the fractional uncertainty in yields for a particular bin of $\left(x_{f}, p_{T}\right)$ is equal to the fractional uncertainty in the production cross-section in that bin. From this a series of weights can be produced allowing the neutrinos from hadrons of particular $p_{t}$ and $x_{f}$ values to be weighted up or down, which will have an effect on the corresponding neutrino energy spectrum at both detectors. Thus, a covariance matrix on the $\mathrm{F} / \mathrm{N}$ ratio can be created [153]. The uncertainty on the FD and ND energy spectrum and on the $\mathrm{F} / \mathrm{N}$ ratio for the $\mathrm{CC}$ sample is shown in figure 6.9 and the same set of uncertainties for the NC sample are shown in figure 6.10.

After the completion of the analysis presented in this thesis the MINER $\nu \mathrm{A}$ experiment [154] released a re-analysis of the NuMI flux [155] showing the NuMI flux to be over-estimated by around $10 \%$ accompanied by modification in the shape of the neutrino flux spectrum. The official result of the re-analysis of the NuMI flux used thin-target data opposed to long-target data (as used by the NuMI facility) due to having a better agreement with the NuMI flux simulation. The effect of the change in the NuMI flux on the analysis in this thesis is negligible due to the ND CC and NC hadron production uncertainties being large enough to cover the differences as shown in figures 6.9 and 6.10 .

\subsection{Beam Focusing}

The NuMI beam uses parabolic focusing horns to direct the charged hadrons into a $675 \mathrm{~m}$ decay pipe. Any mis-modelling in the simulation used to focus the hadrons will have an effect on the shape of the neutrino energy spectrum. 


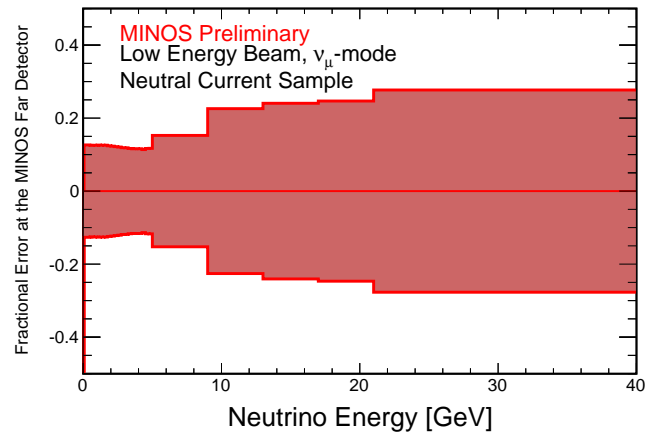

(a) FD NC hadron production error band

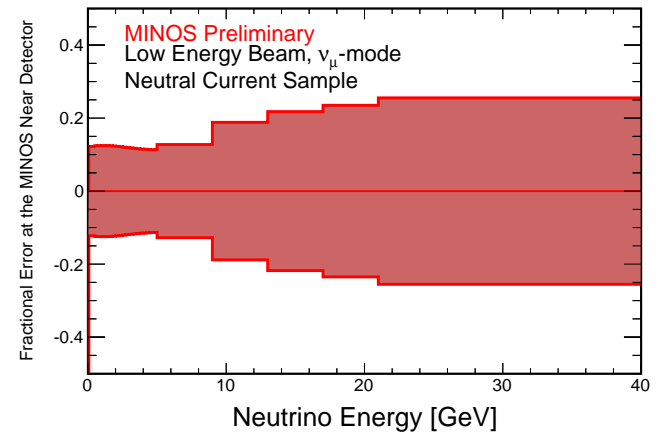

(b) ND NC hadron production error band

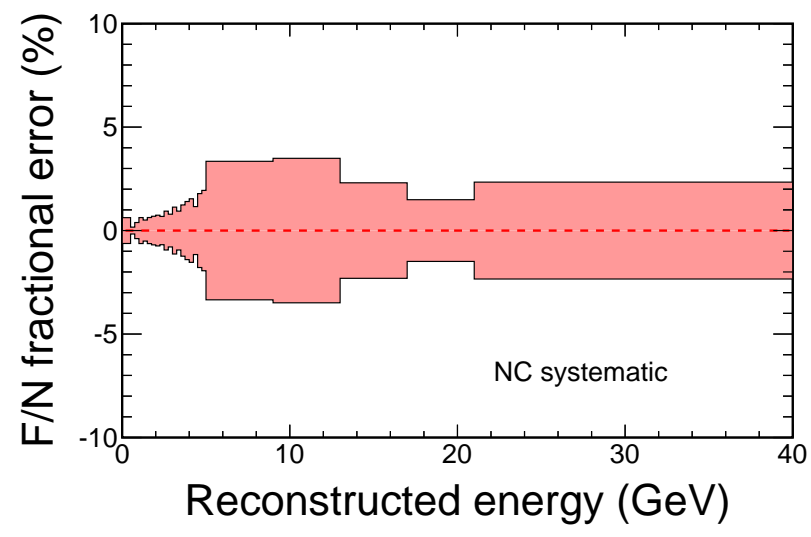

(c) F/N NC hadron production error band

Figure 6.10: The hadron production uncertainty error band as a function of reconstructed neutrino energy for the FD energy spectrum (a), ND energy spectrum (b) and the $\mathrm{F} / \mathrm{N}$ ratio (c) for the $\mathrm{NC}$ sample. The $\mathrm{F} / \mathrm{N}$ error band is the uncertainty used in the sterile neutrino analysis.

Due to these systematic uncertainties affecting both detectors in the same way, the uncertainties on the $\mathrm{F} / \mathrm{N}$ ratio are relativity small compared to those on the FD and ND spectra individually. The following beam-focusing uncertainties were evaluated to produce a covariance matrix on the $\mathrm{F} / \mathrm{N}$ ratio for $\mathrm{CC}$ and $\mathrm{NC}$ events:

1. Horn current mis-calibration: This uncertainty aries due to the error on the calibration of the horn current scale. Any uncertainty on the absolute value of the horn current will have an effect on the focusing of all particles. Direct measurement of the current on the horn was performed with the details found in reference [156]. The results show that the actual horn 
current compared to that used in MC was off by a factor of $1 \%$.

2. Horn current distribution: The magnetic field inside the parabolic focusing horns is produced by sending pulses of current along the surfaces of the inner and outer conductors of the parabolic horns, such that the magnetic field strength is a function of the current, $B(I)$. Thus, when a particle travels through the air gap within the horns, the value of the current, $I$, is the nominal value of $185 \mathrm{kA}$. However, when a particle traverses the inner conductor of the horn the exact value of the current to be used in simulation becomes non-trivial. This uncertainty is expected to affect a very specific portion of the neutrino spectrum, namely that portion coming from pions that spend a great deal of time traversing the horns inner conductors.

For a real conductor with finite conductivity the current penetrates into the bulk by some distance $\delta$. In the beam simulation the horn current is uniformly distributed throughout the conductor and is the same along the length of the horn; this corresponds to the case of a conductor with $\delta=\infty$ (compare this to an ideal conductor which would have all current on the surface, $\delta=0)$. In reality the effective skin depth has been estimated to be $\sim 7 \mathrm{~mm}$ [114]. To produce an uncertainty on this effect two $\mathrm{F} / \mathrm{N}$ ratios in true neutrino energy were produced using the simulation for the cases of $\delta=\infty$ and $\delta=6 \mathrm{~mm}$. The difference between the two $\mathrm{F} / \mathrm{N}$ ratios in true neutrino energy is taken as the $1 \sigma$ uncertainty from the modelling of the skin depth [114] and is shown in figure 6.11.

3. Horn-1 offset: Relative transverse misalignment of the magnetic horns with respect to the target affects the focusing of neutrino parents. When calculating the flux the assumption was made that the horns are perfectly aligned relative to the target. In reality the alignment is not perfect [157]. Various combinations of moving the two horns relative to the target to 
assess the distortion of the $\mathrm{F} / \mathrm{N}$ ratio were performed, in particular varying the transverse alignment for horn-1 and horn-2 to the beam as discussed in detail in reference [114]. The largest change in the $\mathrm{F} / \mathrm{N}$ ratio arises from a mis-alignment of horn-1 (the horn closest to the NuMI target). To evaluate a systematic uncertainty on the $\mathrm{F} / \mathrm{N}$ ratio horn- 1 was shifted in the direction transverse to the beam line by $1 \mathrm{~mm}$. The resulting distortion in the $\mathrm{F} / \mathrm{N}$ ratio was taken to be the systematic uncertainty and is shown in figure 6.11.

4. Baffle scraping: The beam spot-size is not point-like and therefore a small fraction of protons hit the horn-protection "baffle" that is situated in front of the NuMI target. The effect of the protons hitting this baffle effectively acts as a second NuMI target. Due to the baffle being positioned further upstream from the detectors than the target the resulting neutrinos originating from proton-baffle interactions will contribute to the spectra observed at both detectors at higher energies. The uncertainty arises due to the error in determining the fraction of protons from the beam hitting the baffle. A study was performed and the uncertainty in the fraction of protons hitting the baffle was estimated to be of the order of $0.25 \%$ [158]. The uncertainty shown in figure 6.11 for the uncertainty on the baffle scraping systematic was evaluated by producing $\mathrm{F} / \mathrm{N}$ spectra while varying the fraction of protons from the NuMI beam interacting with the baffle by $\pm 0.25 \%$ [114].

To produce the covariance matrices for the $\mathrm{CC}$ and $\mathrm{NC}$ samples, the four systematic uncertainties shown in figure 6.11 are converted into uncertainties on the reconstructed energy spectra for both the CC and NC samples, where most of the effect for the $\mathrm{NC}$ selection will be washed out due to the poor energy resolution. The covariance matrix is computed by assuming $100 \%$ correlation between bins in the $\mathrm{F} / \mathrm{N}$ ratios for each source of uncertainty such that each element $V(i, j)=\sigma_{i} \sigma_{j}$. This results in four covariance matrices for both the CC 


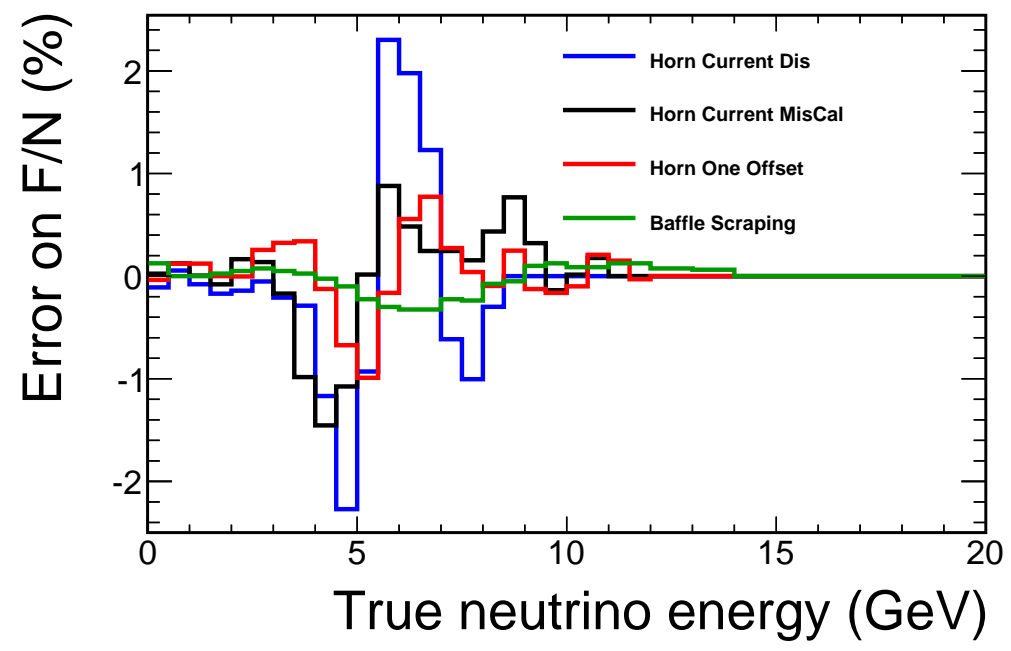

Figure 6.11: The lines show the fractional change in the $\mathrm{F} / \mathrm{N}$ ratio expected in each bin of true neutrino energy due to a one standard deviation shift in various beam parameters. These focusing uncertainties are described in the text and are the input used to generate the covariance matrix for the sterile neutrino analysis. These errors were taken from reference [114].

and NC samples, which are then summed together to give the total covariance matrix associated with the beam focusing uncertainty. The fractional error bands produced from the total covariance matrices for both $\mathrm{CC}$ and $\mathrm{NC}$ events can be seen in figure 6.12 .

\subsection{Total CC and NC Systematic Uncertainties}

The $\mathrm{F} / \mathrm{N}$ fit requires a single total systematic covariance matrix for each of the $\mathrm{CC}$ and NC samples. This is achieved by combining all 26 uncorrelated matrices discussed in this chapter in quadrature for each energy bin and keeping the correlation between bins.

Figures 6.13 and 6.14 show the total covariance matrix used in the $\mathrm{F} / \mathrm{N}$ fit for the sterile neutrino analysis presented in this thesis for both $\mathrm{CC}$ and $\mathrm{NC}$ samples respectively. Up until now only the fractional error band has been shown for each of the main components of the uncertainties since this is the most conceptually useful graph to show (this equates to the square root of the diagonals of the ma- 

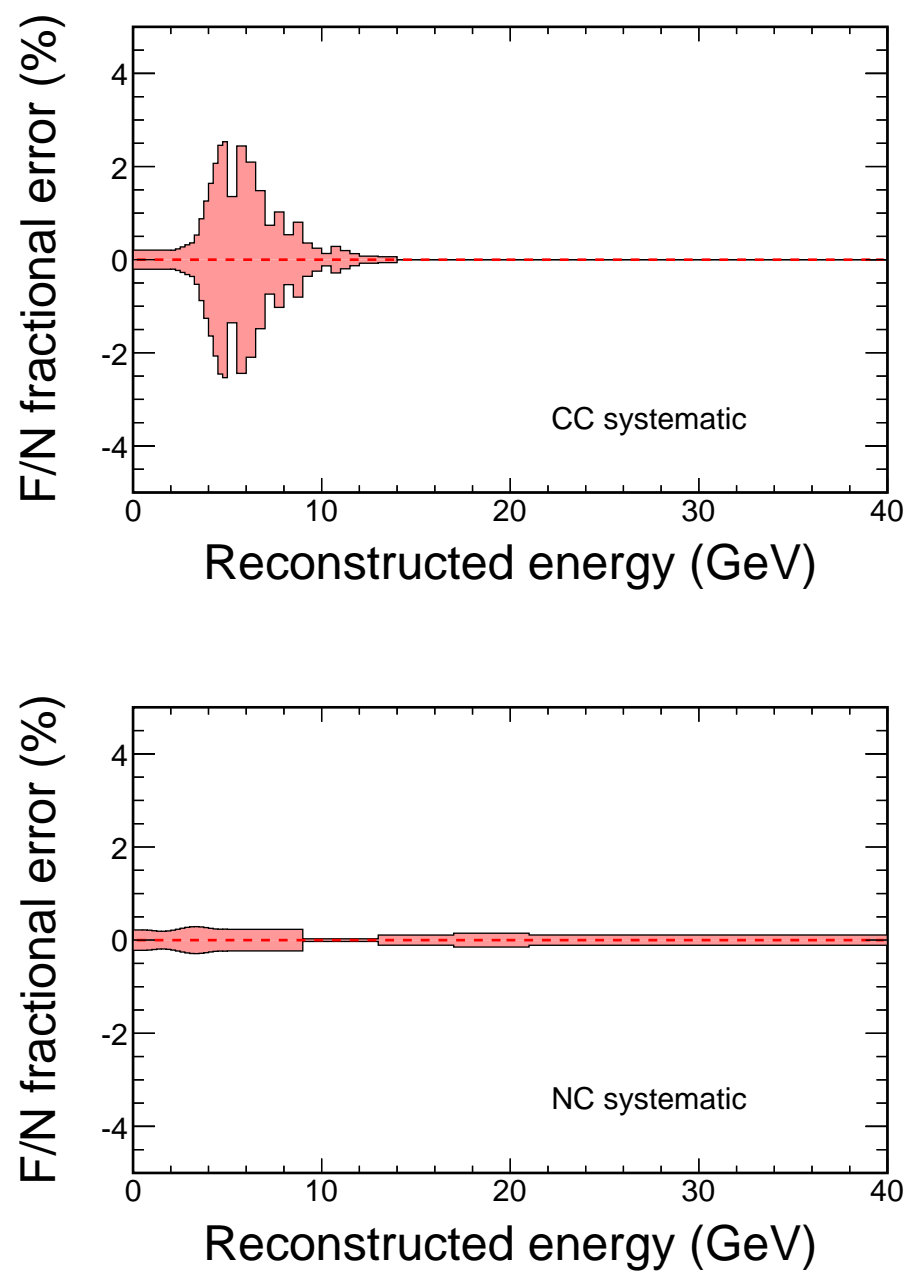

Figure 6.12: Error on the Far-over-Near ratios arising from beam focusing uncertainties for the CC (top) and NC (bottom) samples.

trix). For completeness both the fractional error bands and the total covariance matrices are shown for the $\mathrm{CC}$ and $\mathrm{NC}$ samples in figures 6.13 and 6.14.

The NC and CC total systematic error bands shown in figures 6.13 and 6.14 have their shapes and sizes primarily dominated by the relative normalisation, acceptance and selection efficiency in the ND and the poorly reconstructed events uncertainties described in this chapter. The effects of these systematic uncertainties on the analysis presented in this thesis are shown in section 7.4. 

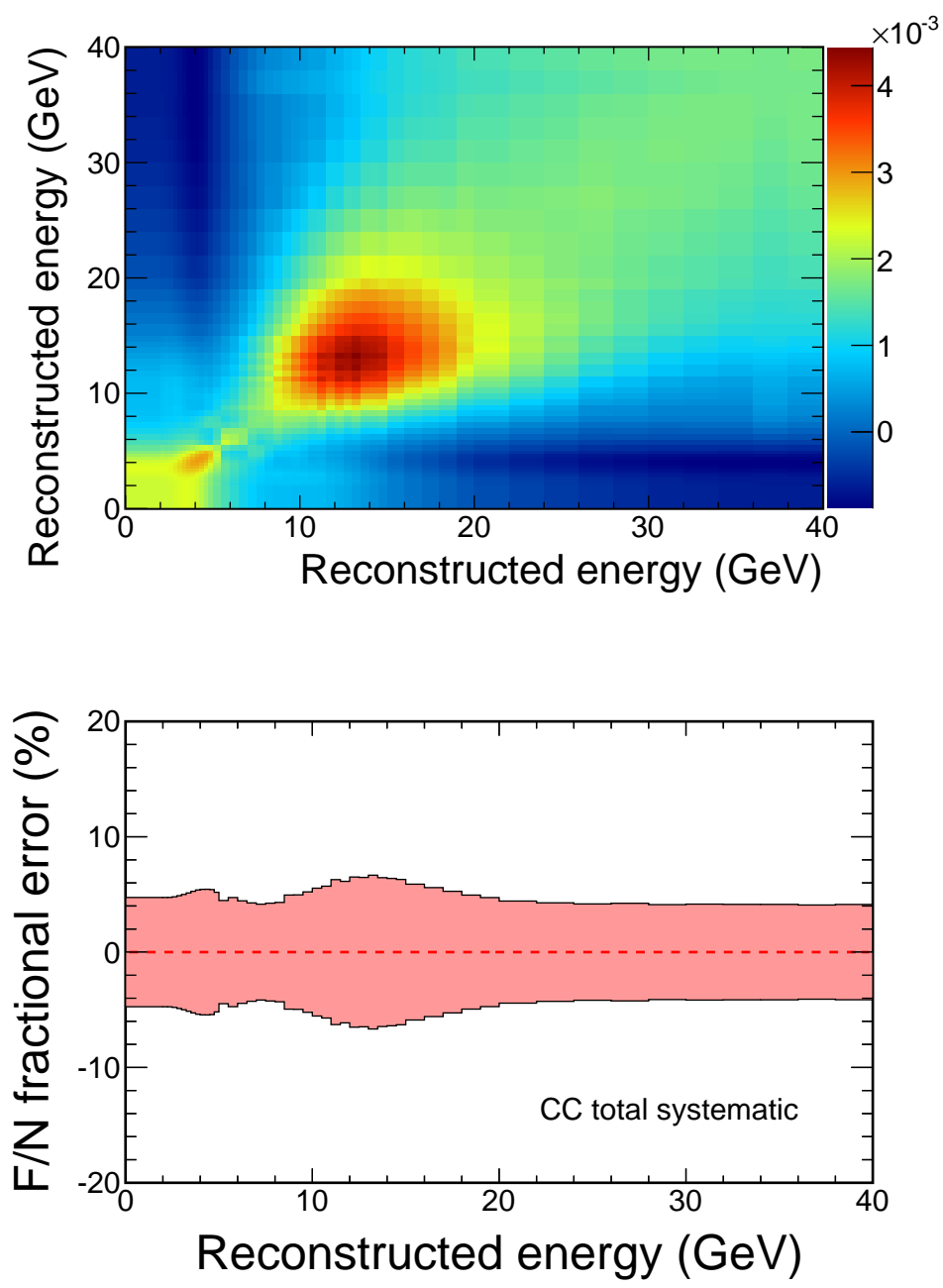

Figure 6.13: Total CC covariance matrix and error band. 

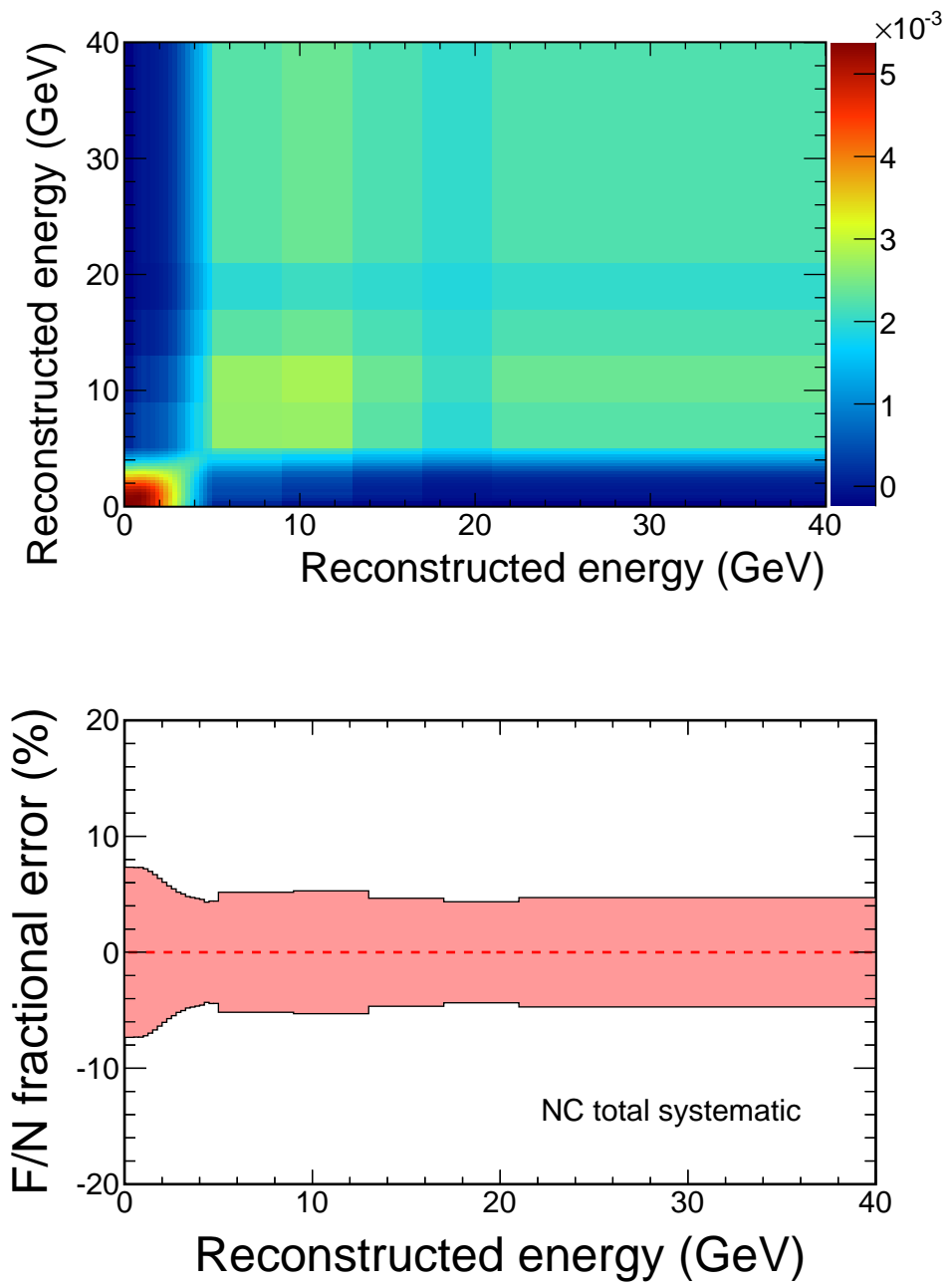

Figure 6.14: Total NC covariance matrix and error band. 


\section{Chapter 7}

\section{The MINOS Sterile Neutrino}

\section{Analysis}

This chapter discusses the physics of the MINOS sterile neutrino analysis and goes on to explain, in detail, the analysis method. A novel technique is discussed using the full $10.56 \times 10^{20} \mathrm{POT} \nu_{\mu}$-running dataset to probe several orders of magnitude in the sterile neutrino parameter space $\left\{\Delta m_{41}^{2}, \sin ^{2} \theta_{24}\right\}$.

\subsection{Sterile Neutrino Oscillations at MINOS}

The MINOS experiment was originally built for the measurement of the threeflavour atmospheric oscillation parameters $\theta_{23}$ and $\Delta m_{32}^{2}$ by looking at $\nu_{\mu}$ disappearance using $\mathrm{CC}$ events with an $L / E$ optimised at $\sim 500 \mathrm{~km} / \mathrm{GeV}$. MINOS went on to make the most precise measurement of the atmospheric mass splitting $\Delta m_{32}^{2}$ and measure the mixing angle $\theta_{23}$, and later on, to identify $\nu_{e} / \bar{\nu}_{e}$ interactions, measure $\theta_{13}$, and obtain some sensitivity to the CP violating phase $\delta_{C P}$. Figure 7.1 shows the three-flavour muon neutrino survival probability along with the electron and tau neutrino appearance probabilities as a function of $L / E$ where $L$ is the distance of the MINOS ND and FD from the NuMI target. Figure 7.1 shows the oscillation maximum expected at the FD in the disappearance of muon 
neutrino events, whilst no oscillations occur at the ND.

The analysis in this thesis uses two channels, the muon survival probability $P\left(\nu_{\mu} \rightarrow \nu_{\mu}\right)$ and sterile neutrino appearance $P\left(\nu_{\mu} \rightarrow \nu_{s}\right)$. From chapter 2 the muon survival probability in a vacuum for $N$ neutrinos can be expressed for $N=4$ as

$$
P\left(\nu_{\mu} \rightarrow \nu_{\mu}\right)=1-4 \sum_{i=1}^{4} \sum_{j<i}^{4}\left|U_{\mu i}\right|^{2}\left|U_{\mu j}\right|^{2} \sin ^{2} \Delta_{j i}
$$

where $\Delta_{j i}=\frac{\Delta m_{j i}^{2} L}{4 E_{\nu}}$, with $L$ defined as the neutrino distance travelled and $E_{\nu}$ is the neutrino energy. The additional mixing between the extra sterile flavour and mass state will alter the muon neutrino survival probability from that of the threeflavour model as shown in figure 7.2 ; it is these deviations from the three-flavour muon-neutrino survival probability that are searched for in this analysis.

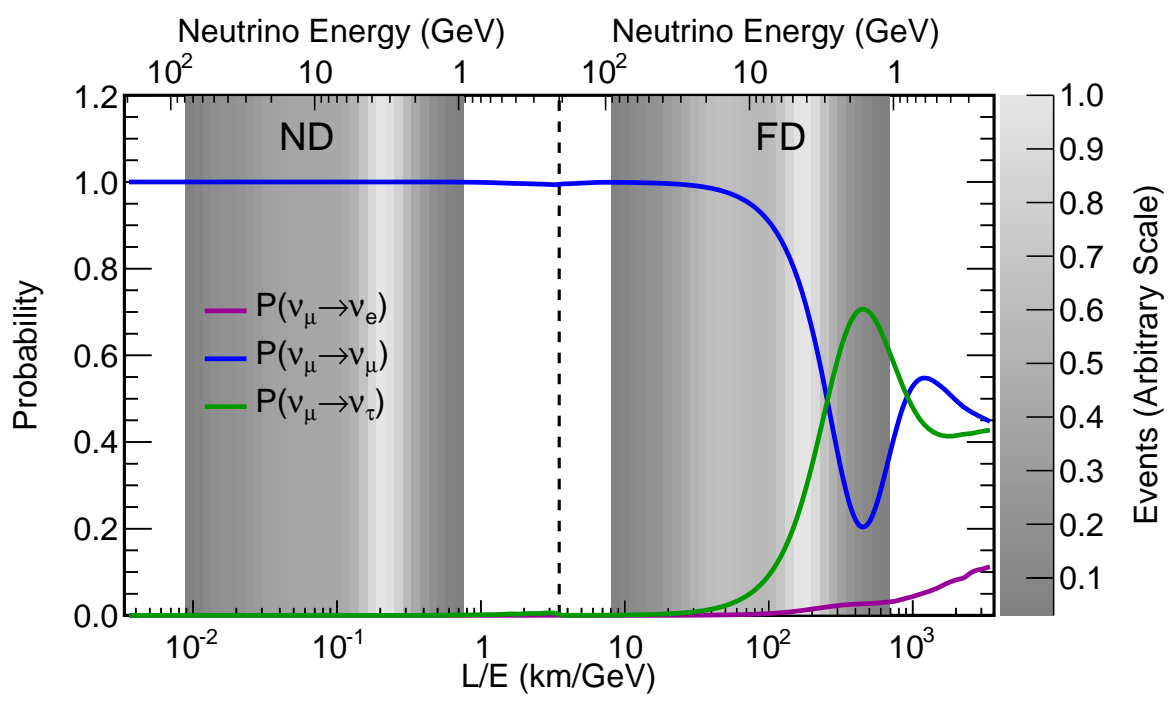

Figure 7.1: The coloured lines indicate the three-flavour oscillation probabilities with no sterile neutrinos as a function of neutrino energy $E$ (top axis) and $L / E$ (bottom axis), where $L$ is either $0.8 \mathrm{~km}$ or $735 \mathrm{~km}$ for the ND and FD respectively. The distance to the ND is calculated to be the average distance due to the possible meson decay position along the $675 \mathrm{~m}$ decay pipe. The grey bands show the regions of $E$ and $L / E$ probed by each detector for $\mathrm{CC} \nu_{\mu}$ events. Values of $\Delta m_{21}^{2}$, $\Delta m_{32}^{2}, \theta_{23}, \theta_{13}$ and $\theta_{12}$ are taken from [28].

The analysis described in this thesis uses the exact probabilities for neutrino oscillations in a vacuum with a four-dimensional PMNS matrix. Figure 7.2 shows 


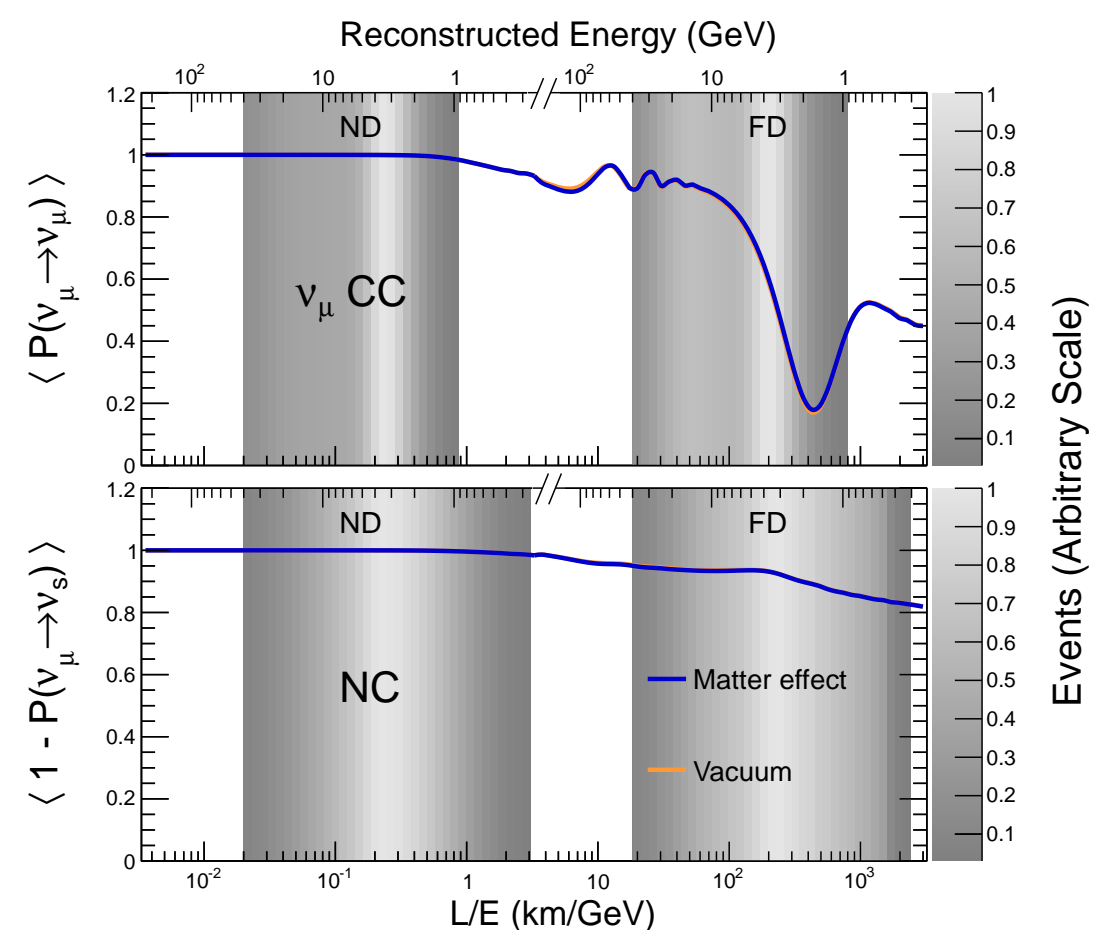

Figure 7.2: Neutrino oscillation probabilities for muon-neutrino survival and sterile neutrino appearance with and without including matter effects. Values of $\Delta m_{21}^{2}, \Delta m_{32}^{2}, \theta_{23}, \theta_{13}$ and $\theta_{12}$ are taken from [28]. The sterile neutrino oscillation parameters used are $\theta_{24}=0.15, \theta_{14}=0.2, \theta_{34}=0.5$. The triangular brackets on the probabilities indicate that the probabilities have been smeared to account for the energy resolution of the MINOS detectors.

the effect of introducing matter effects into the probabilities and how they could impact this analysis. The difference is negligible.

To further the discussion of the phenomenology several approximations are made in this section that are not made in the actual analysis. The solar neutrino terms $\Delta_{12}$ are considered negligible and the approximation $\Delta m_{31}^{2} \approx \Delta m_{32}^{2}$ is made. In the limit $\Delta m_{41}^{2} \gg \Delta m_{31}^{2}$ the approximation $\Delta m_{43}^{2} \approx \Delta m_{42}^{2} \approx \Delta m_{41}^{2}$ is made. The muon-neutrino survival probability in equation 7.1 has terms expanded around $s_{13}, s_{14}, s_{24}$ and $\cos 2 \theta_{23}$, where $c_{i j}=\cos \theta_{i j}$ and $s_{i j}=\sin \theta_{i j}$. This approximation can be expressed as

$$
P\left(\nu_{\mu} \rightarrow \nu_{\mu}\right) \approx 1-\sin ^{2} 2 \theta_{23} \cos 2 \theta_{24} \sin ^{2} \Delta_{31}-\sin ^{2} 2 \theta_{24} \sin ^{2} \Delta_{41},
$$


where equation 7.2 shows that the muon-neutrino survival probability is strongly influenced by the sterile mixing angle $\theta_{24}$. Thus, MINOS becomes sensitive to $\theta_{24}$ by looking at $\nu_{\mu}$-CC and $\bar{\nu}_{\mu}$-CC events. This would be seen as a depletion of muon neutrino events at either of the MINOS detectors, depending on the size of $\Delta m_{41}^{2}$, as the neutrinos oscillate into sterile neutrinos as well as to the other active neutrino flavours.

MINOS is also sensitive to sterile neutrinos through the disappearance of NC events $[147,159,160]$. MINOS is equally sensitive to the NC interactions of all active neutrino flavours, however mixing with sterile flavour states would result in a depletion in the number of $\mathrm{NC}$ events observed in either detector. This process is described by the probability $P\left(\nu_{\mu} \rightarrow \nu_{s}\right)$. By taking the approximations described previously, the probability affecting NC disappearance can be expressed as

$$
1-P\left(\nu_{\mu} \rightarrow \nu_{s}\right) \approx 1-c_{14}^{4} c_{34}^{2} \sin ^{2} 2 \theta_{24} \sin ^{2} \Delta_{41}-A \sin ^{2} \Delta_{31}-B \sin ^{2} 2 \Delta_{31},
$$

where the terms $A$ and $B$ are functions of the mixing angles and phases. To first order, $A=s_{34}^{2} \sin ^{2} 2 \theta_{23}$ and $B=\frac{1}{2} \sin \delta_{24} s_{24} \sin 2 \theta_{34} \sin 2 \theta_{23}$. Equation 7.3 shows dependence on the sterile neutrino oscillation parameters $\theta_{24}, \theta_{34}$ and $\delta_{24}$. Figure 7.3 shows the sensitivity of the NC disappearance channel to various values of the CP violating phases. In each case all other phases are set to zero except the one being varied. It can be seen that the NC disappearance channel exhibits significant sensitivity to $\delta_{24}$. However, sensitivity in this channel is limited by the poor energy resolution (due to the outgoing neutrino) of $\mathrm{NC}$ events and a significant background from $\nu_{\mu}$ and $\nu_{e} \mathrm{CC}$ events; therefore this analysis will make the assumption that $\delta_{14}=\delta_{34}=\delta_{24}=0$.

The sterile mixing angle $\theta_{14}$ does not appear in equation 7.2 due to being a sub-dominant term. It appears in equation 7.3, but in both cases the effect of $\theta_{14}$ only becomes non-negligible at large values of $\theta_{14}$. An analysis of solar and 

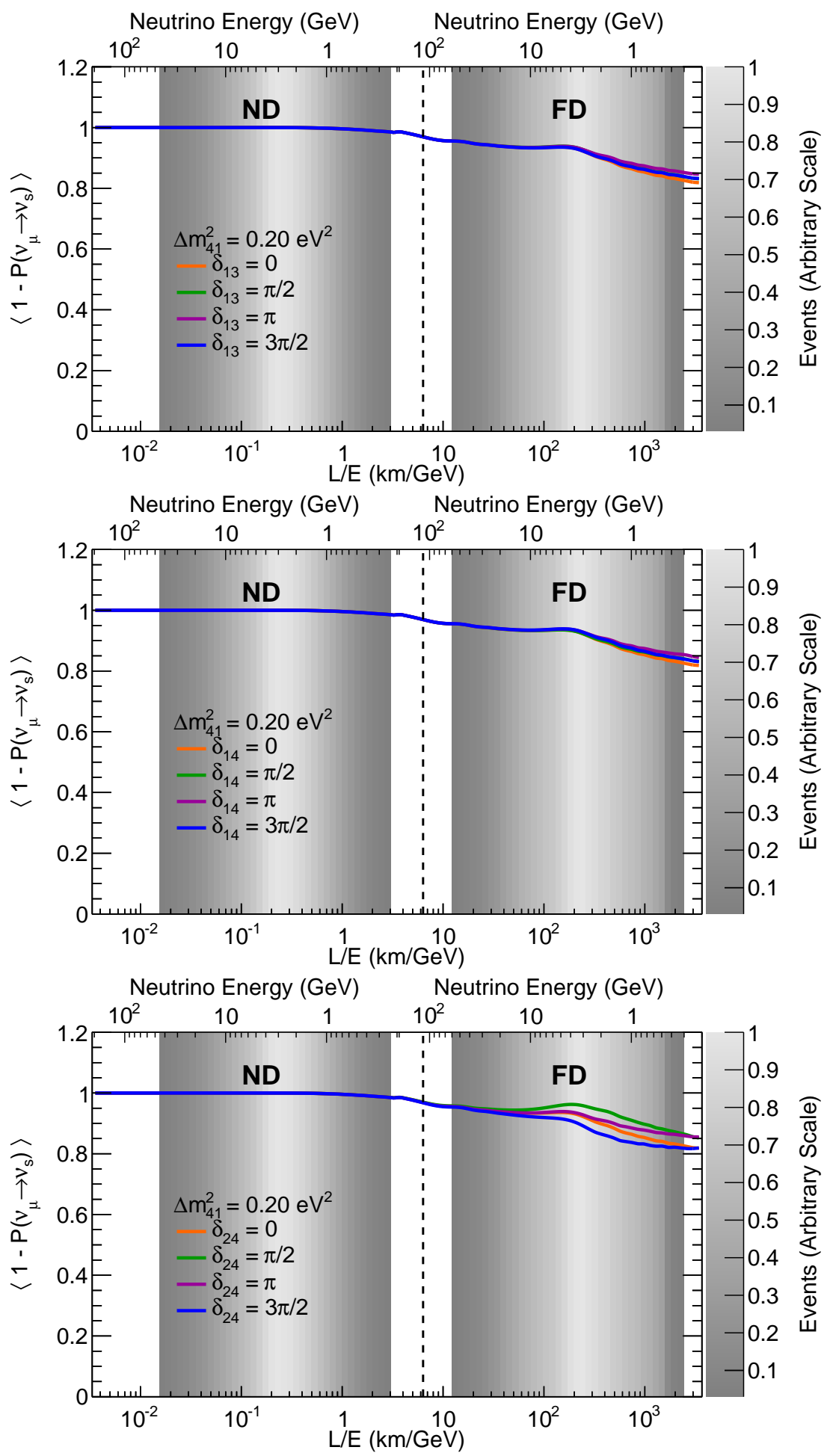

Figure 7.3: The effect of the $\mathrm{CP}$ violating phases in the $3+1$ model on $\left\langle 1-P\left(\nu_{\mu} \rightarrow \nu_{s}\right)\right\rangle$, which effects the reconstructed energy spectrum of NC interactions. The labelled phase in each panel is the only non-zero phase. All other oscillation parameters are fixed in every panel. Values of $\Delta m_{21}^{2}, \Delta m_{32}^{2}, \theta_{23}, \theta_{13}$ and $\theta_{12}$ are taken from [28]. The sterile neutrino oscillation parameters used are $\theta_{24}=0.15, \theta_{14}=0.2, \theta_{34}=0.5$. The triangular brackets on the probabilities indicate that the probabilities have been smeared to account for the energy resolution of the MINOS detectors. 
reactor neutrino data yields the constraint $\sin ^{2} \theta_{14}<0.041$ at $90 \%$ C.L. [161]. Therefore this analysis sets $\theta_{14}=0$, which is interpreted as no mixing between $\nu_{e}$ and $\nu_{s}$, i.e $\left|U_{e 4}\right|^{2}=0$.

Using all the approximations above, the matrix elements of interest to MINOS can be expressed as

$$
\begin{aligned}
& \left|U_{\mu 4}\right|^{2}=\sin ^{2} \theta_{24}, \\
& \left|U_{\mu 3}\right|^{2}=\cos ^{2} \theta_{13} \cos ^{2} \theta_{24} \sin ^{2} \theta_{23}, \\
& \left|U_{\tau 4}\right|^{2}=\cos ^{2} \theta_{24} \sin ^{2} \theta_{34}, \\
& \left|U_{s 4}\right|^{2}=\cos ^{2} \theta_{24} \cos ^{2} \theta_{34}, \\
& \left|U_{s 3}\right|^{2}=-\cos ^{2} \theta_{13}\left(\cos \theta_{34} \sin \theta_{23} \sin \theta_{24}+\cos \theta_{23} \sin \theta_{34}\right)^{2} .
\end{aligned}
$$

\subsection{The MINOS Neutrino Energy Spectra}

\subsubsection{The MINOS Dataset Epoch}

The MINOS-era data are recorded in run periods; over the duration of the MINOS experiment the NuMI beam has operated predominantly in the low-energy configuration with the horn polarity in the forward setting which gives a predominantly muon-neutrino dominated beam. For a fuller explanation of the NuMI beam configurations see chapter 3 . The analysis described in this thesis uses run periods $1,2,3,5,6$ and 10, corresponding to a protons-on-target (POT) exposure of $10.56 \times 10^{20}$ at the FD. Table 7.1 shows the run periods taken in MINOS and the polarity of the NuMI beam for that run period along with the POT of good-quality data recorded at the FD. Note that the ND POT does not match the FD exposure, since the dataset is closed early at the ND to allow time for data quality and validation checks. Due to the high statistics at the ND due to 


\begin{tabular}{|c|c|c|c|}
\hline Run & Configuration & Horn polarity & Good data FD POT $\left(\times 10^{18}\right)$ \\
\hline \hline 1 & LE & Forward & 126.93 \\
1 & pHE & Forward & 15.31 \\
2 & LE & Forward & 194.27 \\
3 & LE & Forward & 388.71 \\
4 & LE & Forward & 8.84 \\
4 & LE & Reverse & 170.85 \\
5 & LE & Forward & 45.89 \\
6 & LE & Forward & 61.62 \\
7 & LE & Reverse & 124.08 \\
8 & LE & Forward & 12.58 \\
9 & LE & Reverse & 40.80 \\
10 & LE & Forward & 238.31 \\
\hline \hline \multicolumn{2}{|c|}{ Total good physics data } \\
\hline$\nu_{\mu}$ beam run periods $(1,2,3,5,6,10)$ & 1428.19 \\
$\nu_{\mu}$ LE beam run periods $(1,2,3,5,6,10)$ & 1071.04 \\
\hline \multicolumn{2}{|c|}{ beam run periods $(4,7,9)$} & 1055.73 \\
\hline
\end{tabular}

Table 7.1: Summary of the data collected in MINOS in terms of the POT exposure. The majority of the data comes from a low-energy (LE) beam with an energy peak around $3 \mathrm{GeV}$. A small fraction of the data, not used in this analysis, comes from a pseudo high energy beam (pHE) with an energy peak around $7 \mathrm{GeV}$. Table taken from [162].

the increased flux rate the main goal is to keep the MINOS FD live for as long as possible.

\subsubsection{Inter-Detector Flux Differences}

MINOS relies on the two-detector setup, where both detectors are composed of the same technology, materials and similar electronics such that a relative comparison between the neutrino energy spectra in the two detectors allows for a significant cancellation of systematics that would be large effects in the individual detectors, for example uncertainties in neutrino cross-sections, the neutrino flux prediction from the NuMI beam, hadronic shower modelling and track energy measurements from range.

This cancellation of systematics would be exact if the normalised flux experienced at both the ND and FD was identical. For MINOS this is not the case. 
Due to parent decay kinematics the neutrino flux each detector experiences is different. Figure 7.4 shows the neutrino parents as they decay along the $675 \mathrm{~m}$ NuMI beam decay pipe. The FD, being $735 \mathrm{~km}$ away, subtends a significantly smaller solid angle to the neutrino parent than the ND. Only one direction for the neutrino parent to decay will allow the resultant neutrino to pass through the FD. The ND is only $\sim 1 \mathrm{~km}$ away from the NuMI hadron production target and therefore a range of decay angles allow a neutrino to pass through the detector.

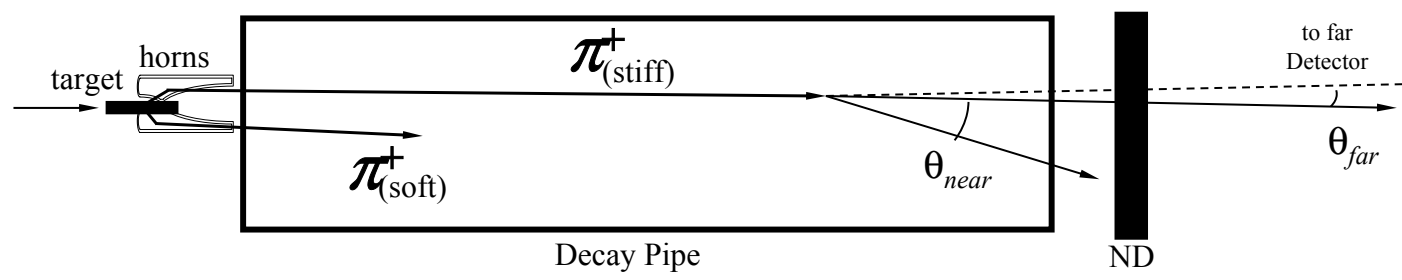

Figure 7.4: A diagram (not to scale) of neutrino parents in the NuMI decay pipe illustrating the different solid angles subtended by the ND and FD at the parent decay point.

\subsubsection{Far Detector Extrapolation}

Traditionally, MINOS analyses have used the ND data shown in figure 7.5 to characterise the NuMI beam and correct the MC simulation. From this, a predicted FD reconstructed energy spectrum can be obtained.

Various extrapolation techniques have been explored to predict the FD energy spectrum given the ND energy spectrum. The simplest method is the Far/Near method. This uses the discrepancies between the ND data/MC ratio as a function of the reconstructed neutrino energy to re-weight the reconstructed neutrino energy spectrum of the simulated FD. The predicted number of events in reconstructed energy bin $i$ at the FD is given as

$$
F_{i}^{\text {Prediction }}=N_{i}^{\text {Data }} \times \frac{F_{i}^{\mathrm{MC}}}{N_{i}^{\mathrm{MC}}}
$$



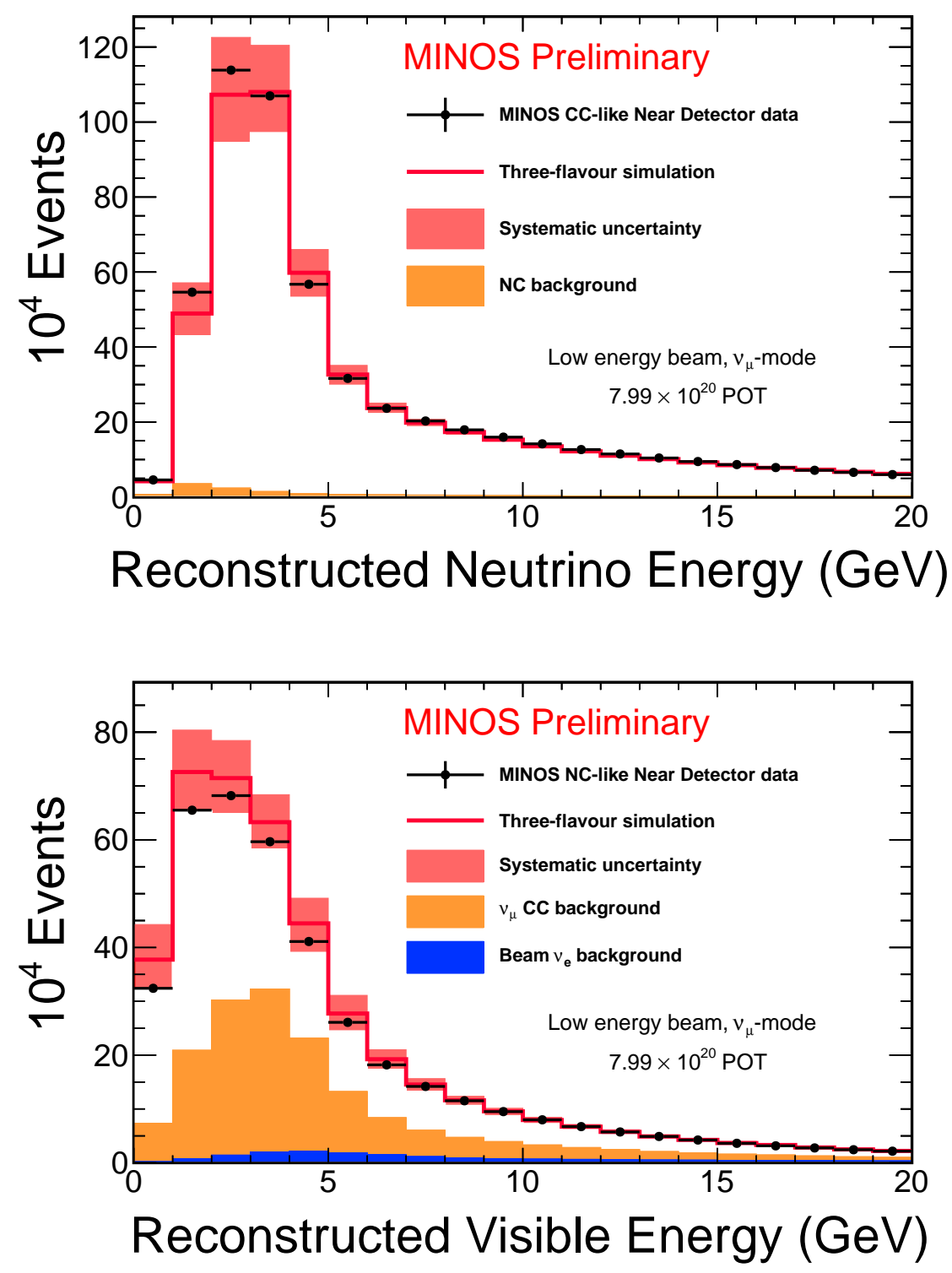

Figure 7.5: The CC (top) and NC (bottom) reconstructed neutrino energy spectra at the ND. The red histograms in both the CC and NC panels are the three-flavour simulated spectra with the systematic error bands included. In both cases the relevant backgrounds have been calculated from simulation and are included. 
where $F_{i}^{\text {Prediction }}$ is the prediction of the FD reconstructed neutrino energy in bin $i, F_{i}^{\mathrm{MC}}$ and $N_{i}^{\mathrm{MC}}$ are the simulated FD and ND energy spectra in bin $i$ and $N_{i}^{\text {Data }}$ is the number of observed events in bin $i$. The Far/Near method assumes that the relationship between true and reconstructed neutrino energy between the two detectors is identical as well as assuming that the detectors have the same energy resolution and same selector efficiency for the data sample. A previous study into sterile neutrino oscillation at MINOS for a fixed value of $\Delta m_{43}^{2}=0.5 \mathrm{eV}^{2}$ used the Far/Near extrapolation method [163].

A more robust extrapolation is the beam matrix method. This has been the primary extrapolation method for many previous MINOS analyses, e.g. the latest measurement of the three-flavour oscillation parameters [79]. It is considered more robust, as this multistage process considers the differences between the two detectors such as purity, sample background and differences in energy resolution. It also encodes the differences in the shape of the neutrino flux arising from the solid angle considerations discussed in section 7.2.2. A more detailed explanation of the beam matrix method can be found in [127].

\subsubsection{Neutral Current $R$-Values at the Far Detector}

We can use the Far/Near extrapolation method to make an initial quantitative statement about the level of sterile-neutrino-driven disappearance of NC events. To do this, we make a prediction of the NC energy spectrum at the FD assuming standard three-flavour oscillation parameter-values for $\Delta m_{32}^{2}$ and $\theta_{23}$ taken from [79], $\theta_{13}$ from a weighted average of reactor measurements [164, 165, 166], and $\Delta m_{21}^{2}$ and $\theta_{12}$ from [167]. This prediction is shown in the bottom panel of figure 7.6. We take this prediction and compare it to the data in table 7.2.

To quantify the agreement between the observed and expected NC energy spectra at the FD, a metric $R$ is defined as 

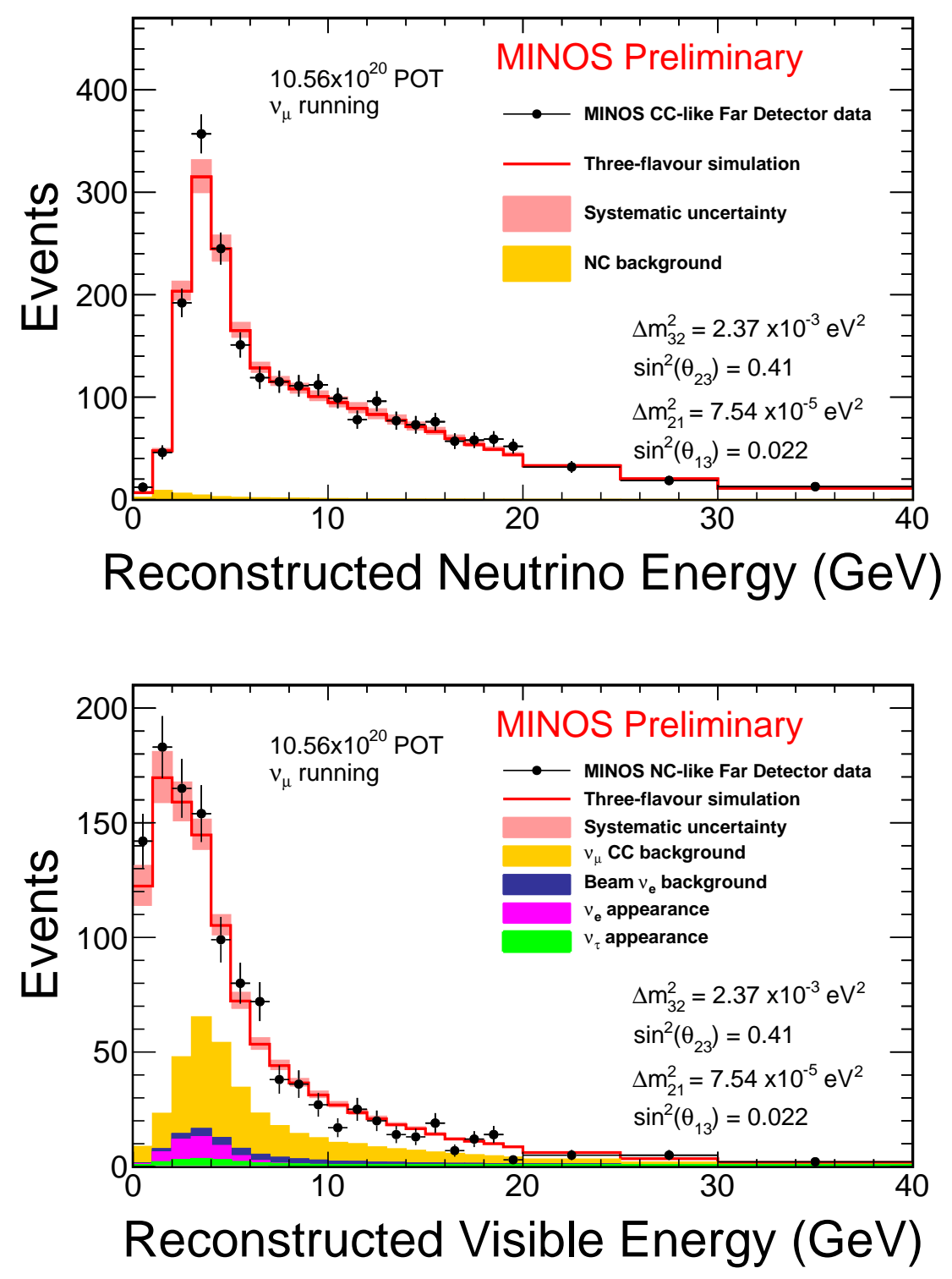

Figure 7.6: The CC (top) and NC (bottom) reconstructed neutrino energy spectra at the FD. The red histograms in both the $\mathrm{CC}$ and NC panels are the three-flavour simulated spectra for the extrapolated FD prediction with the Far-over-Near ratio systematic error band included. The values used for the three-flavour oscillation parameters are taken from the latest MINOS three-flavour analysis [79]. In both cases the relevant backgrounds have been calculated from simulation and are included. 


\begin{tabular}{c||c|c||c|c}
\hline \hline Energy (GeV) & FD CC Data & FD CC Pred & FD NC Data & FDNC Pred \\
\hline $0-40$ & 2563 & 2539 & 1211 & 1175 \\
$0-3$ & 250 & 266 & 490 & 455 \\
$3-40$ & 2313 & 2258 & 721 & 718 \\
\hline
\end{tabular}

Table 7.2: The observed number of neutrino events at the FD compared to the expected number assuming a three-flavour model. Three different energy regions were defined, motivated by the $\mathrm{NC}$ neutrino spectrum having a large number of $\mathrm{NC}$ events at low energies. Events above $40 \mathrm{GeV}$ are not considered in this thesis.

$$
R=\frac{N_{\mathrm{data}}-\sum B_{\mathrm{CC}}}{S_{\mathrm{NC}}},
$$

where $N_{\text {data }}$ is the integrated number of FD data events in the $0-40 \mathrm{GeV}$ energy range. Throughout this thesis, we do not use any neutrino events with energies above $40 \mathrm{GeV}$ due to the poorly-understood discrepancies with the simulation and associated large systematics uncertainties. The integrated total background in the NC sample, taken from simulation, is $\sum B_{\mathrm{CC}} . S_{\mathrm{NC}}$ is the total predicted number of true NC events. The $R$-value is calculated over three different energy ranges motivated by the shape of the NC energy spectrum. The values of $R$ are given in table 7.3, along with statistical and systematic uncertainties. For this calculation, the systematic uncertainties are taken to be a $15 \%$ normalisation on the CC background events and a 5.3\% normalisation on the NC signal events.

\begin{tabular}{c|c|c|c|c}
\hline \hline Energy $(\mathrm{GeV})$ & $R$-value & \pm syst & \pm stats & \pm total \\
\hline $0-40$ & 1.049 & 0.095 & 0.045 & 0.105 \\
$0-3$ & 1.100 & 0.073 & 0.061 & 0.095 \\
$3-40$ & 1.008 & 0.128 & 0.067 & 0.144 \\
\hline
\end{tabular}

Table 7.3: $R$-values calculated from the Far Detector neutral current neutrino energy spectrum.

The $R$-value is defined such that an exact agreement between data and the three-flavour prediction would yield $R=1$, and it can be seen in table 7.3 that the observed $R$-values are consistent with this. We can interpret this as no 
observed deficit of NC events driven by sterile neutrinos. Since this calculation makes no assumption about the number of sterile neutrinos, we can view this as a model-independent statement, not tied to the $3+1$ phenomenological model used elsewhere in this thesis.

The top panel of figure 7.6 compares the $\mathrm{CC}$ data at the FD to the predicted CC energy spectrum (using the Far/Near extrapolation), with table 7.2 showing the event counts. Whilst no model-independent $R$-value can be defined for these $\nu_{\mu}$ flavour-tagged events, we can qualitatively view the good agreement between data and prediction as an indicator that no anomalous sterile-neutrino-driven disappearance of CC events has been seen.

\subsection{Fitting with the Far-over-Near Ratio}

\subsubsection{Near Detector Oscillations}

The MINOS concept of extrapolation discussed in section 7.2.3 assumes that the $\mathrm{ND}$ is situated in a region of $L / E$ such that it is unaffected by neutrino oscillations. However, the analysis in this thesis considers a $3+1$ model where the additional mass splitting, $\Delta m_{41}^{2}$, is unknown, and could be anywhere in the range $10^{-3}-10^{2} \mathrm{eV}^{2}$. Once $\Delta m_{41}^{2}>1 \mathrm{eV}^{2}$, the neutrino oscillation probability becomes non-zero in the region of $L / E$ probed by the ND; thus both $\nu_{\mu}$ and $\bar{\nu}_{\mu}$ disappearance and sterile neutrino appearance could occur at the ND. Figure 7.7 shows the oscillation probability for muon (anti)neutrino disappearance (top panel) for increasing values of $\Delta m_{41}^{2}$. The bottom panel shows the sterile neutrino appearance probability (therefore disappearance of $\mathrm{NC}$ events) for various values of $\Delta m_{41}^{2}$. The possible appearance or disappearance of neutrinos at the ND makes the aforementioned procedures to extrapolate from the ND to create a FD prediction invalid.

To deal with oscillations in the $\mathrm{ND}$, the $\mathrm{F} / \mathrm{N}$ ratio for the data is fit directly 


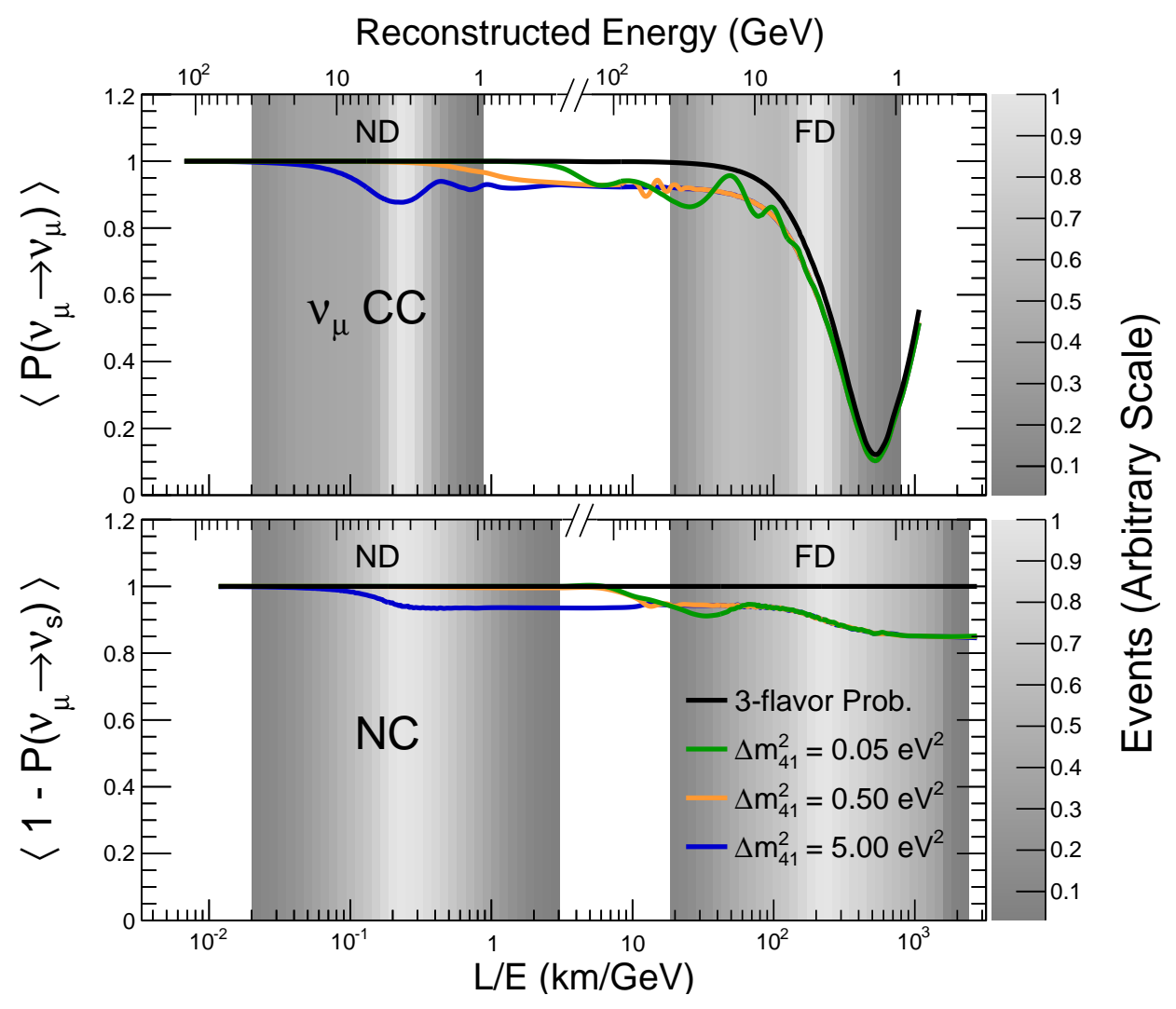

Figure 7.7: The $\nu_{\mu}$-CC (top) and NC (bottom) disappearance probabilities as a function of $L / E$ for various values of $\Delta m_{41}^{2}$. The figure illustrates how, for large values of $\Delta m_{41}^{2}$, the traditional FD extrapolation techniques used by MINOS in previous analyses will no longer work since oscillations begin to affect the ND. Values of $\Delta m_{21}^{2}, \Delta m_{32}^{2}, \theta_{23}, \theta_{13}$ and $\theta_{12}$ are taken from [28]. The sterile neutrino oscillation parameters used are $\theta_{24}=0.15, \theta_{14}=0.2$ and $\theta_{34}=0.5$, and all CP phases are set to zero. The triangular brackets on the probabilities indicate that the probabilities have been smeared to account for the energy resolution of the MINOS detectors.

against the simulated $\mathrm{F} / \mathrm{N}$ ratio, over the parameter space $\left\{\Delta m_{41}^{2}, \sin ^{2} \theta_{24}\right\}$. Figure 7.8 shows the $\mathrm{CC}$ and $\mathrm{NC} \mathrm{F} / \mathrm{N}$ ratios in the energy window $0-40 \mathrm{GeV}$ which is used in the fit. By fitting the $\mathrm{F} / \mathrm{N}$ ratio directly, the systematic uncertainties mis-modelled in both detectors are cancelled. However, the beam weights that are usually derived from the ND data can no longer be applied, since their derivation implicitly assumes that no sterile neutrinos are causing oscillations in the ND, and the untuned beam simulation must be taken as the nominal. The beam systematics largely cancel, and a hadron production error is assessed and included into the fit as was discussed in section 6.4. 


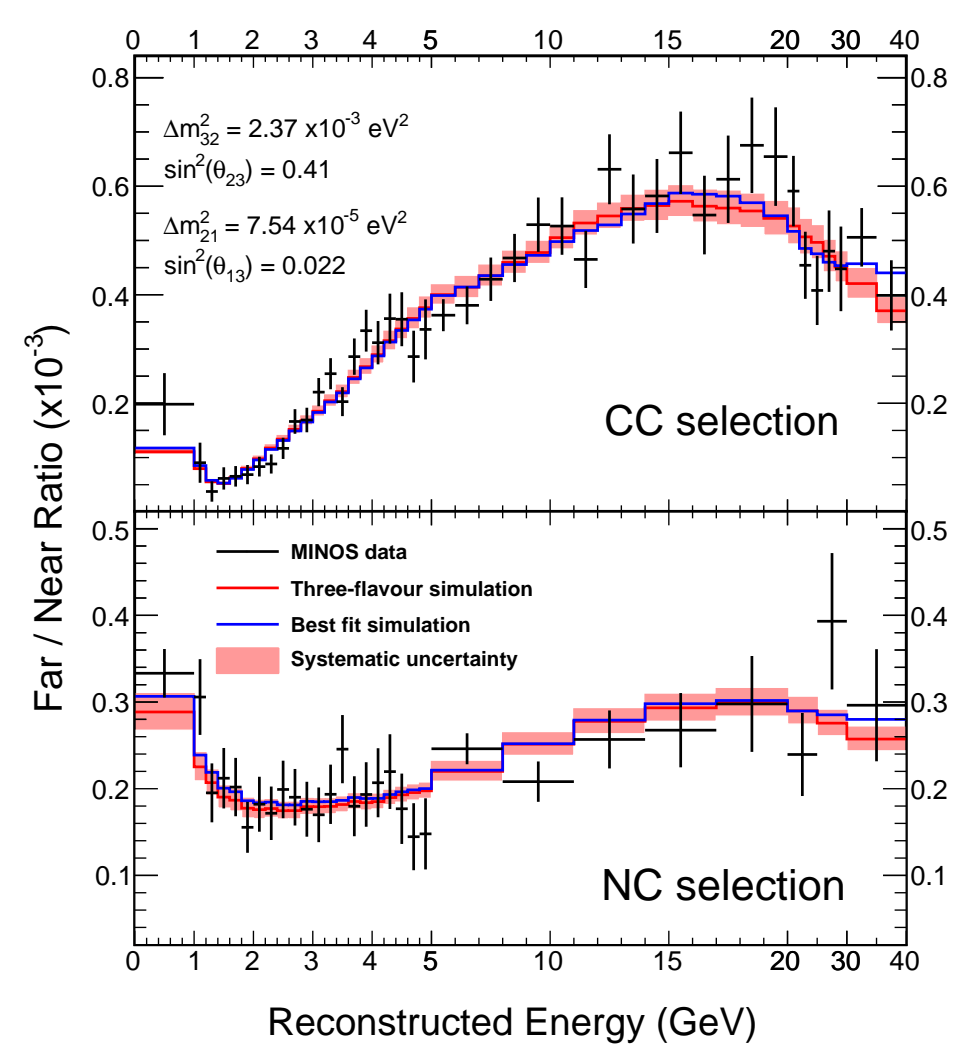

Figure 7.8: The F/N ratio for $\nu_{\mu}$-CC events (top panel) and for NC events (bottom panel). The black points are the MINOS data observed in the $10.56 \times 10^{20} \mathrm{POT}$ exposure. The red band is the systematic uncertainty on the $\mathrm{F} / \mathrm{N}$ ratio taken from the covariance matrix used in the fit as described in chapter 6 . The red line is the standard three-flavour oscillation simulation (the null hypothesis) with values of $\Delta m_{21}^{2}, \Delta m_{32}^{2}, \theta_{23}, \theta_{13}$ and $\theta_{12}$ taken from [28]. The blue line is the best-fit simulation from the analysis discussed in section 7.3.3.

In previous analyses the MINOS simulation would be oscillated using probabilities for the various components of the the FD spectrum as a function of neutrino energy $E$, where $L$ would be taken as a fixed value of $735 \mathrm{~km}$. Although the neutrino parents can decay anywhere from the production target up to the end of the $675 \mathrm{~m}$ decay pipe the maximum fractional variation in neutrino distance travelled to the FD is given as $\delta L / L \sim 1 \times 10^{-3}$. However, assuming oscillations at the ND, which is only a baseline of $1.04 \mathrm{~km}$, results in a maximum fractional variation in path length of $\delta L / L \sim 0.65$ that therefore needs to be accommodated. The neutrino oscillation probabilities are therefore calculated as a function of $L / E$, where the distance travelled by the neutrino from parent 


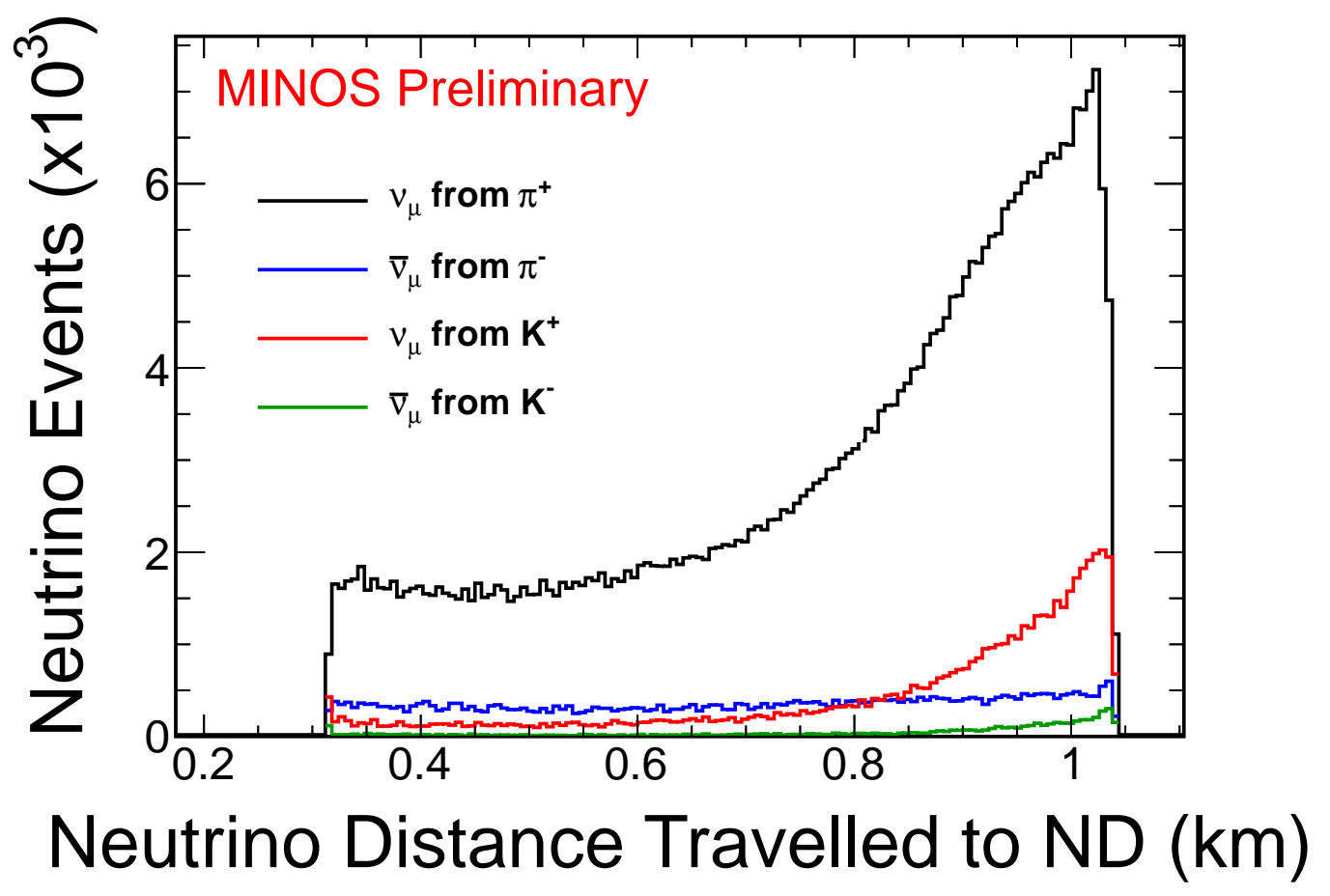

Figure 7.9: The distance travelled by neutrino that interact inside the ND. Note the target is at the far right and the end of the decay pipe is at the far left. To explain further, events that are produced at the target will have travelled the full $1.04 \mathrm{~km}$ to the ND, hence why the target is on the right of this plot.

decay point until interaction in the MINOS detectors is taken from simulation. Figure 7.9 shows the number of neutrinos and anti-neutrinos as a function of total distance travelled to the ND, where a significant number of events have travelled a distance shorter than the nominal $1.04 \mathrm{~km}$.

\subsubsection{The $\chi^{2}$ Expression}

Instead of incorporating systematics as nuisance parameters, this analysis elected to incorporate them as systematic uncertainties on the $\mathrm{F} / \mathrm{N}$ ratio in each bin of energy used in the fit using the covariance matrix described in chapter 6 . The value of the observed $\mathrm{F} / \mathrm{N}$ ratio is denoted by $\mathbf{x}$ and the simulated $\mathrm{F} / \mathrm{N}$ prediction as a function of the oscillation parameters is $\boldsymbol{\mu}$ with both being dimension $N$, where $N$ is the total number of reconstructed energy bins (48 bins for the CC 
$\mathrm{F} / \mathrm{N}$ and 24 bins for the $\mathrm{NC} \mathrm{F} / \mathrm{N})$. The assumption that the likelihood, $L$, of a measurement $\mathbf{x}$ given a prediction, $\boldsymbol{\mu}$, is distributed by a multivariate Gaussian expressed as

$$
L(\mathbf{x} ; \boldsymbol{\mu}, V)=\frac{1}{(2 \pi)^{N / 2}|V|^{1 / 2}} \exp \left[-\frac{1}{2}(\mathbf{x}-\boldsymbol{\mu})^{T} V^{-1}(\mathbf{x}-\boldsymbol{\mu})\right]
$$

where $V$ is the $N \times N$ covariance matrix containing the 26 combined statistical and systematic uncertainties and $|V|$ is the determinant of the covariance matrix $V$. The covariance matrix is defined as

$$
V=V^{\mathrm{stat}}+\sum_{i=1}^{26} V_{i}^{\mathrm{syst}}
$$

Chapter 6 describes in detail the creation of the systematic component of this matrix for the $\mathrm{NC}$ and $\mathrm{CC} \mathrm{F} / \mathrm{N}$ ratios. The statical component is by definition a diagonal matrix (statistical errors are not correlated bin-to-bin) with a statistical error $\sigma_{i}^{2}$ for an energy bin $i$,

$$
V^{\text {stat }}=\left(\begin{array}{ccc}
\sigma_{1}^{2} & \ldots & 0 \\
\vdots & \ddots & \vdots \\
0 & \cdots & \sigma_{N}^{2}
\end{array}\right)
$$

The statistical error in the $\mathrm{FD}$ and $\mathrm{ND}, \sigma_{F}$ and $\sigma_{N}$, are taken as square root of the predicted number of events for each bin, $\sqrt{F}$ and $\sqrt{N}$. Using error propagation the error on the $\mathrm{F} / \mathrm{N}$ ratio, $\sigma(F / N)$, becomes

$$
\begin{aligned}
\sigma^{2}(F / N) & =\left(\frac{F}{N}\right)^{2}\left[\left(\frac{\sigma_{F}}{F}\right)^{2}+\left(\frac{\sigma_{N}}{N}\right)^{2}\right] \\
& =\left(\frac{F}{N}\right)^{2}\left[\left(\frac{\sqrt{F}}{F}\right)^{2}+\left(\frac{\sqrt{N}}{N}\right)^{2}\right], \\
& =\frac{F}{N^{2}}+\frac{F^{2}}{N^{3}}
\end{aligned}
$$


In the limit of infinite statistics at the ND, the second term in equation 7.14 can be approximated to zero and the expression for the statistical uncertainty on the $\mathrm{F} / \mathrm{N}$ ratio becomes

$$
\sigma(F / N) \approx \frac{\sqrt{F}}{N}
$$

The likelihood $L$ is then computed and values of the oscillation parameters found that maximise this likelihood. The equivalent approach of minimising the $\chi^{2}$ distribution was taken as expressed below:

$$
\chi^{2}=(\mathbf{x}-\boldsymbol{\mu})^{T} V^{-1}(\mathbf{x}-\boldsymbol{\mu})+\frac{\left(N_{D}-N_{M C}\right)^{2}}{\sigma_{N D}^{2}}+\frac{\left(\left|\Delta m_{32}^{2}\right|-\Delta m^{2}\right)^{2}}{\sigma_{\Delta m^{2}}^{2}} .
$$

The second term in equation 7.16 provides a constraint on the absolute neutrino flux at the ND, where $N_{D}$ and $N_{M C}$ represent the integrated ND reconstructed neutrino energy spectrum for the $0-40 \mathrm{GeV}$ energy window used in the fit. Neutrino flux estimations have large uncertainties associated with them, and the error on the ND flux was conservatively set such that $\sigma_{N D}=50 \% N_{M C}$. The final term in equation 7.16 is a penalty term on the atmospheric mass splitting parameter $\Delta m_{32}^{2}$. This term provides a weak constraint such that within the fit, its value is centred around the standard three-flavour oscillation value. The justification for this penalty term is the that this analysis looks for any perturbative deviations from the three-flavour neutrino model, which can already describe the MINOS data well. Any large deviations due to the $3+1$ model that would require $\Delta m_{32}^{2}$ to have a significantly different value would have already shown up. The penalty term's central value $\Delta m^{2}=2.5 \times 10^{-3} \mathrm{eV}^{2}$ is taken from the reactor experiment Daya Bay using their three-flavour analysis results [168], and the uncertainty $\sigma_{\Delta m^{2}}^{2}=0.5 \mathrm{eV}^{2}$ corresponds to roughly twice the uncertainty on the Daya Bay result. The first two terms in equation 7.16 sum over the bins in both the CC and $\mathrm{NC}$ F/N ratios.

The fit minimises the quantity in equation 7.16 , which assumes that both the 
statistical and systematic fluctuations follow a Gaussian distribution. Neutrino physics experiment are mostly statistically limited in their results, following a more discrete Poisson nature for low $N$. For this analysis to ensure a good Gaussian approximation the binning of the FD energy spectrum used in the fit (the ND is assumed to have infinite statistics) is chosen such that all bins in the three-flavour prediction contain at least 15 events for the $10.56 \times 10^{20} \mathrm{POT}$ exposure. This allows one to treat the $\chi^{2}$ as a likelihood defined as $\chi^{2}=-2 \ln L$.

\subsubsection{Performing the Fit}

The fit was performed using the MINUIT package [169] to minimise the $\chi^{2}$ expression from equation 7.16. The fit is performed such that a two dimensional likelihood surface is constructed in the plane $\left\{\Delta m_{41}^{2}, \sin ^{2} \theta_{24}\right\}$. Therefore a grid is constructed in the range $\Delta m_{41}^{2} \in\left[10^{-4}, 10^{2}\right] \mathrm{eV}^{2}$ and $\theta_{24} \in[0, \pi / 2]$ radians. At each point in this grid the values of $\Delta m_{41}^{2}$ and $\theta_{24}$ are kept fixed. The remaining parameters that MINOS is sensitive to are $\Delta m_{32}^{2}, \theta_{23}$ and $\theta_{34}$; these are allowed to vary during the fit and the best fit value is chosen such that the $\chi^{2}$ is minimised. The fit enforces constraints on the mixing angles such that only physical values are allowed, abiding by the parameterisation of the PMNS matrix stated in section 7.1. The constraints imposed are $\left\{\theta_{23}, \theta_{34}\right\} \in[0, \pi / 2]$. All CP-violating phases are set to zero, as is $\theta_{14}$. The justification for this was described earlier. The number of $\mathrm{CC}-\nu_{e}$ interactions arising from $\nu_{e}$ appearance account for $2.9 \%$ of the NC sample at the FD, which is itself smaller than the statistical and systematic uncertainties on that sample. By setting $\sin ^{2} \theta_{14}=0.041$, the upper limit from reactor constraints [161], this $2.9 \%$ contribution decreases by $4 \%$. Overall, this becomes a negligible $0.14 \%$ change to the total NC sample, hence why this analysis can justify setting $\theta_{14}$ to zero. The parameter $\Delta m_{32}^{2}$ is varied but is constrained by the penalty term in equation 7.16. The solar parameters are fixed to global values [167] and $\theta_{13}$ is taken from the weighted average of Daya Bay, 
Double Chooz and RENO measurements $[164,165,166]$. A summary of the fit constraints and fixed values are displayed in table 7.4.

The fit takes into account both the normal and inverted mass orderings (the sign of $\Delta m_{32}^{2}$ ) along with both the lower and upper octants of $\theta_{23}$. This requires the fit to be performed four times for the same grid point in the plane $\left\{\Delta m_{41}^{2}, \sin ^{2} \theta_{24}\right\}$ with the lowest $\chi^{2}$ out of the four to be chosen as the best fit.

\begin{tabular}{c|c|c}
\hline \hline Variable & Free/Fixed & Constraint \\
\hline$\Delta m_{41}^{2}$ & Fixed & $\in\left[10^{-4}, 10^{2}\right] \mathrm{eV}^{2}$ \\
$\theta_{24}$ & Fixed & $\in[0, \pi / 2]$ \\
$\Delta m_{32}^{2}$ & Free & Penalty term, see Eq. 7.16 \\
$\left\{\theta_{34}, \theta_{23}\right\}$ & Free & $\in[0, \pi / 2]$ \\
$\left\{\theta_{14}, \delta_{13}, \delta_{14}, \delta_{14}\right\}$ & Fixed & 0 \\
$\Delta m_{21}^{2}$ & Fixed & $7.54 \times 10^{-5} \mathrm{eV}^{2}$ \\
$\theta_{12}$ & Fixed & 0.554 \\
$\theta_{13}$ & Fixed & 0.149
\end{tabular}

Table 7.4: Values and constraints on neutrino oscillations parameters in the $3+1$ fit.

\subsubsection{The Likelihood Surfaces}

Figure 7.10 shows the likelihood surface for the MINOS dataset from fitting a $3+1$ sterile neutrino model. Figure 7.11 shows the individual contributions from the $\mathrm{CC}$ and $\mathrm{NC}$ samples used in the fit. The global best fit is represented by a yellow star in both figures and the traditional method of producing $90 \%$ C.L. contours is performed using the global-scan method in a parameter space with two degrees of freedom. The contour contains all $\chi^{2}$ values such that $\chi^{2}-\chi_{\min }^{2}>4.61$ (the origin of 4.61 is explained in section 8.3 ), where $\chi_{\min }^{2}$ is the minimum $\chi^{2}$ value in that respective surface. A global minimum can be seen in figure 7.10. The values of the $3+1$ parameters at this best fit are shown in table 7.5. Figure 7.10 shows the best fit point at a high value of the sterile neutrino mass splitting, 
$\Delta m_{41}^{2}\left(\mathrm{eV}^{2}=64.58\right.$ and a non-zero value for the mixing angle $\theta_{24}$. Features in the $\mathrm{F} / \mathrm{N}$ ratio can give rise to a global minimum (best fit point) with non-zero sterile neutrino psicalltion parameter values. This could be contributed by to a small systematic shape change in reconstructed neutrino energy or a statistical fluctuation in the FD data. To access the significance of any such global minimum and the effect this has on the traditional confidence interval construction methods the Feldman-Cousins unified approach [170] is implemented and is discussed in detail in chapter 8.

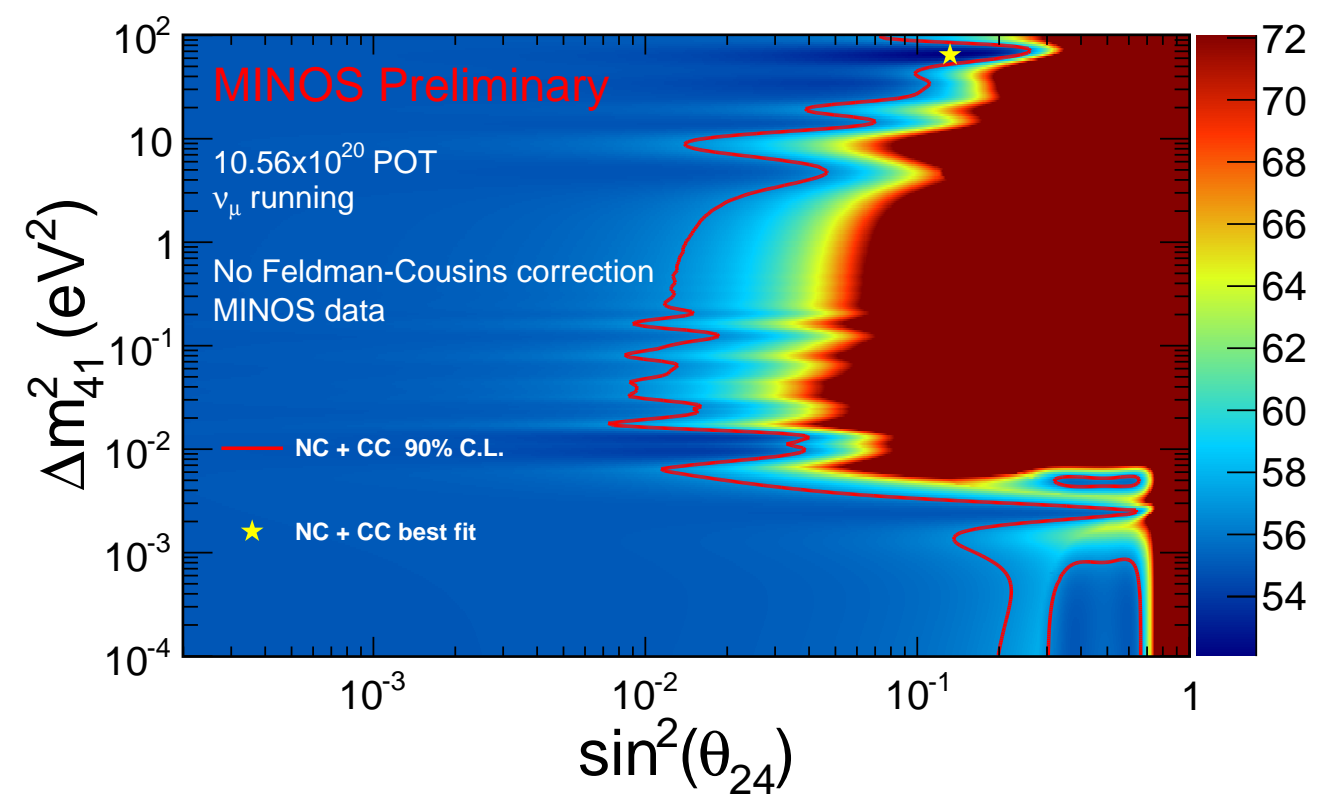

Figure 7.10: Likelihood surface for the MINOS NC and CC samples. The red line indicates a confidence contour using the global scan method for a $90 \%$ C.L. for two degrees of freedom, i.e. it is drawn at $\chi_{\min }^{2}+4.61$. The yellow star indicates the best fit for the parameters $\Delta m_{41}^{2}$ and $\theta_{24}$. The $z$-axis has been restricted to show a colour gradient for values of $\chi_{\min }^{2} \rightarrow \chi_{\min }^{2}+20$.

\subsubsection{Degeneracies in the $3+1$ Sterile Neutrino Model}

The likelihood surfaces in figures 7.10 and 7.11 show some interesting features that arise due to degenerate effects within the $3+1$ model. For particular sets of non-zero parameters in the $3+1$ model, the oscillations probabilities tend towards 

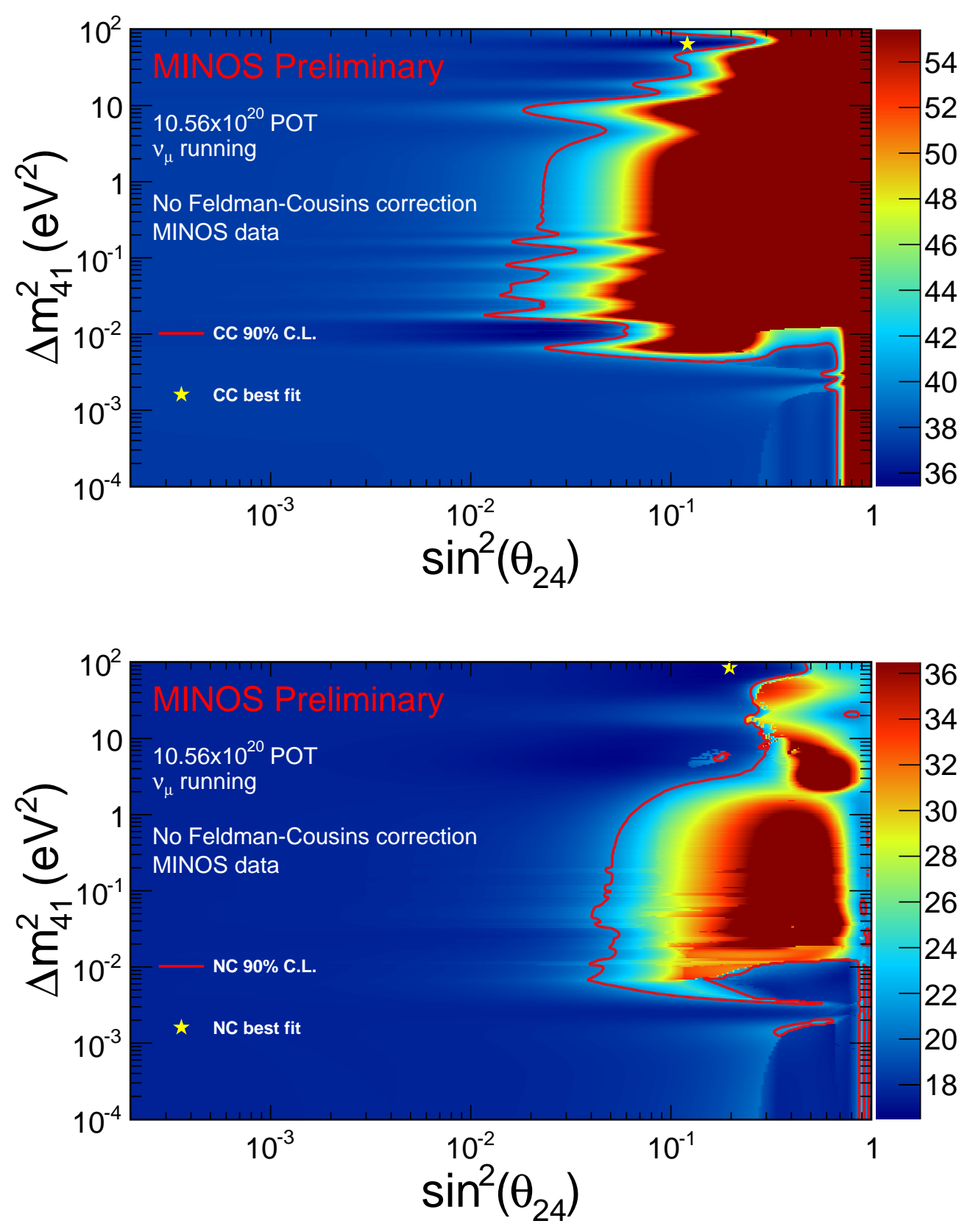

Figure 7.11: Likelihood surface from the MINOS CC (top) and NC (bottom) separately. The red lines indicate confidence contours using the global-scan method for a $90 \%$ C.L. for two degrees of freedom, i.e. it is drawn at $\chi_{\min }^{2}+4.61$. The yellow star indicates the best fit for the parameters $\Delta m_{41}^{2}$ and $\theta_{24}$. The $z$-axis has been restricted to show a colour gradient for values of $\chi_{\min }^{2} \rightarrow \chi_{\min }^{2}+20$.

values such that the model can become indistinguishable from the null hypothesis, where the null hypothesis is defined as all sterile parameters set to zero values. These degenerate features can be described as follows: 


\begin{tabular}{|c|c|}
\hline Parameter & Best Fit Value \\
\hline \hline$\Delta m_{32}^{2}\left(10^{-3} \mathrm{eV}^{2}\right)$ & 2.41 \\
$\Delta m_{41}^{2}\left(\mathrm{eV}^{2}\right)$ & 64.58 \\
$\theta_{23}$ & 0.724 \\
$\theta_{24}$ & 0.373 \\
$\theta_{34}$ & 0.0474 \\
\hline
\end{tabular}

Table 7.5: Summary of the best fit values at the combined charged-current and neutral-current global minimum fitting the Far-over-Near ratio against a $3+1$ model.

- $\Delta m_{41}^{2} \approx 2 \Delta m_{31}^{2}$. In this case, $\Delta m_{43}^{2}=\Delta m_{31}^{2}$ and so $\Delta m_{41}^{2}$ and $\Delta m_{31}^{2}$ can both contribute to the standard atmospheric scale oscillation dip. For values of $\theta_{24} \approx \pi / 4$ the fit will converge on values of $\left\{\theta_{34}, \theta_{23}\right\} \approx \pi / 2$, which allow for the probability distributions to mimic those of the three-flavour case.

- $\Delta m_{41}^{2} \approx \Delta m_{31}^{2}$. In this case $\Delta m_{43}^{2}=0$, and the mixing of muon neutrinos into sterile neutrinos is occurring at the same wavelength as the traditional three-flavour mixing of muon neutrinos into tau neutrinos. MINOS struggles to distinguish these two effects for $\theta_{24}<\pi / 4$ (lower octant). For $\theta_{24}>\pi / 4$ by looking at equation 7.2 one can see that the $\cos 2 \theta_{24}$ term becomes negative. Thus, as $\theta_{24}$ varies from $\pi / 4 \rightarrow \pi / 2$ the amount of sterile mixing decreases as well as suppressing the three-flavour mixing. The data shows a clear depletion of muon neutrinos at the FD and therefore rules out large values of $\theta_{24}$.

- $\Delta m_{41}^{2} \ll \Delta m_{31}^{2}$. In this case $\Delta m_{43}^{2}=\Delta m_{31}^{2}$. This is similar to the first condition, however now, any terms containing $\Delta m_{41}^{2}$ become very small. For values of $\theta_{24} \approx \pi / 4$ the fit will converge on values of $\left\{\theta_{34}, \theta_{23}\right\} \approx \pi / 2$, which allows for the probability distributions to mimic those of the threeflavour case. 


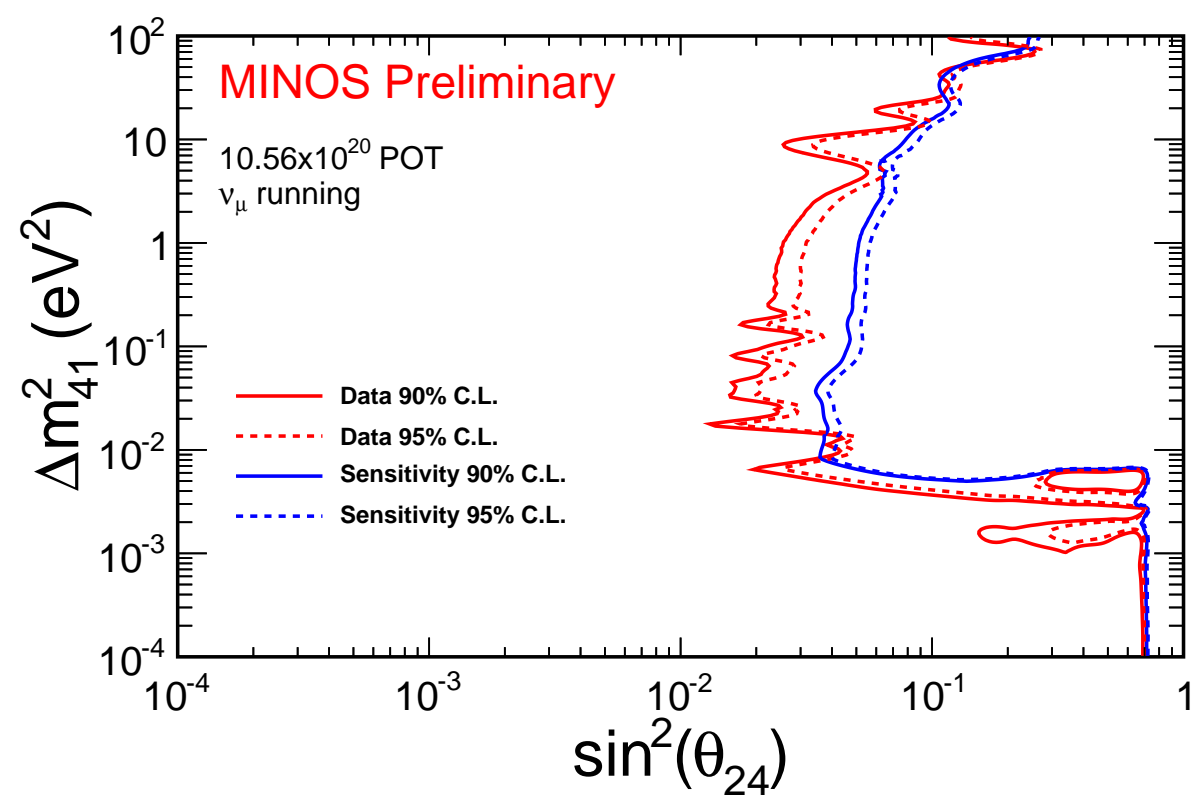

Figure 7.12: The $90 \%$ and $95 \%$ C.L. for the MINOS data and sensitivity in the plane $\left\{\Delta m_{41}^{2}, \sin ^{2} \theta_{24}\right\}$, calculated using the unified procedure of Feldman and Cousins and incorporates all sources of systematic uncertainty.

The conditions stated above come about due to the relationship between $\Delta m_{41}^{2}$ and $\Delta m_{31}^{2}$. In this analysis the fit has the freedom to vary $\Delta m_{31}^{2}$, and therefore to achieve the smallest $\chi^{2}$ possible that can satisfy the above conditions as often as possible. The penalty term on $\Delta m_{31}^{2}$ penalises any value of $\Delta m_{31}^{2}$ away from the central value of $\Delta m^{2}=2.5 \times 10^{-3} \mathrm{eV}^{2}$. This has the effect of only allowing these degenerate cases to occur at this mass splitting scale or below.

\subsubsection{Confidence Limits with Exact Coverage}

The contours shown in figures 7.10 and 7.11 are constructed such that $\chi^{2}-$ $\chi_{\min }^{2}>4.61$, where $\chi_{\min }^{2}$ is the minimum $\chi^{2}$ value in that respective surface. Under the assumption of Gaussian statistics, one would interpret these confidence intervals as 90\% C.L.. Chapter 8 will go into detail about why in this analysis this interpretation is invalid and provides incorrect limits. At each point in parameter space we interpret the significance of the $\Delta \chi^{2}$ with respect to the global minimum according to the unified procedure of Feldman and Cousins [170]. Figure 7.12 


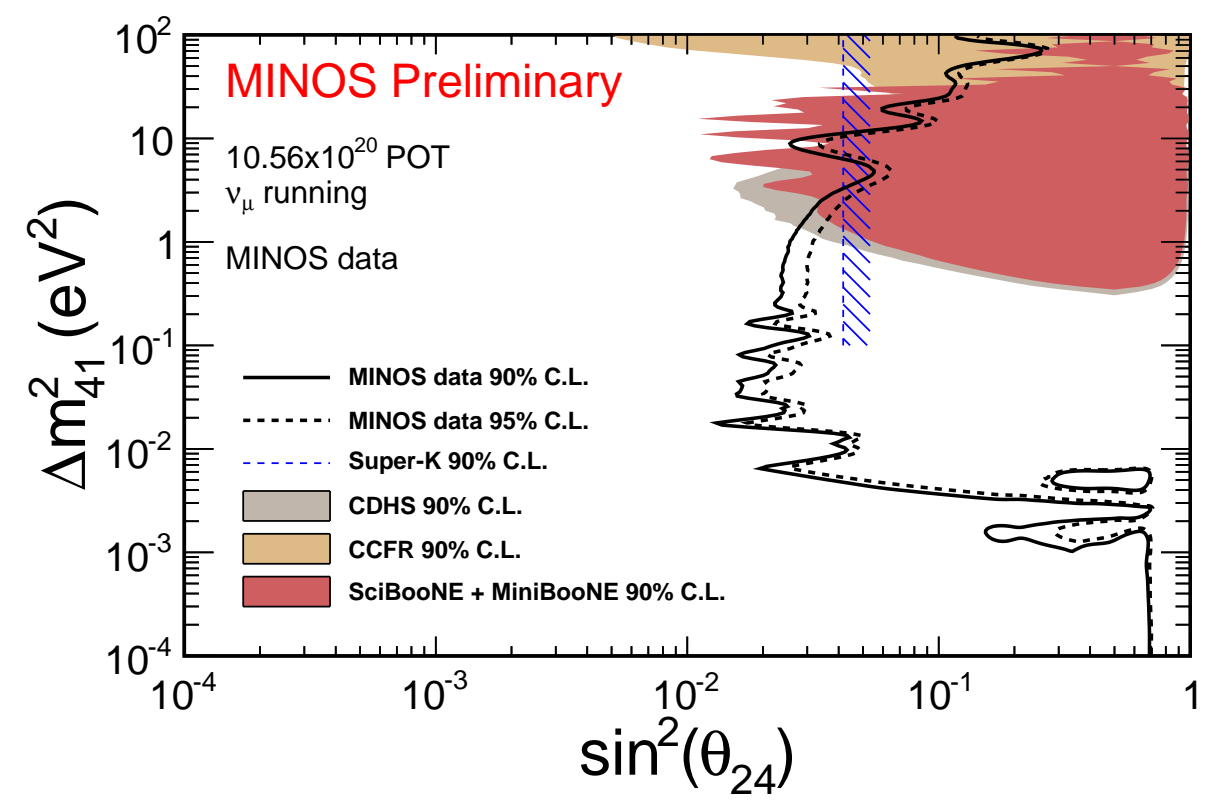

Figure 7.13: Showing the MINOS $90 \%$ and $95 \%$ C.L. compared to other $\nu_{\mu}$ disappearance measurements $[171,172,173,174]$. The MINOS limits here are produced using the unified procedure of Feldman and Cousins and incorporates all sources of systematic uncertainty.

shows the $90 \%$ and $95 \%$ C.L. for the MINOS data and sensitivity where both the CC and NC samples were fit together. The confidence limits in this figure have been produced according to the unified procedure of Feldman and Cousins and therefore provide exact coverage.

Figure 7.13 compares this result with similar previous experimental results to see how consistent the result is and how it contributes to the greater understanding of the topic of sterile neutrinos. Figure 7.13 shows the MINOS data for both $90 \%$ and $95 \%$ C.L in the plane $\Delta m_{41}^{2}-\sin ^{2} \theta_{24}$ compared to other experimental limits. The other experimental limits are obtained through looking at the $\nu_{\mu}$-CC disappearance channel and are therefore sensitive mainly to $\theta_{24}$, making these results a good comparison to the results in this thesis. The results shown in figure 7.13 are from Super-Kamiokande [171], CDHS [172], CCFR [173] and MiniBooNE and SciBooNE [174].

The limits shown in figure 7.13 are all obtained from measurements looking at 


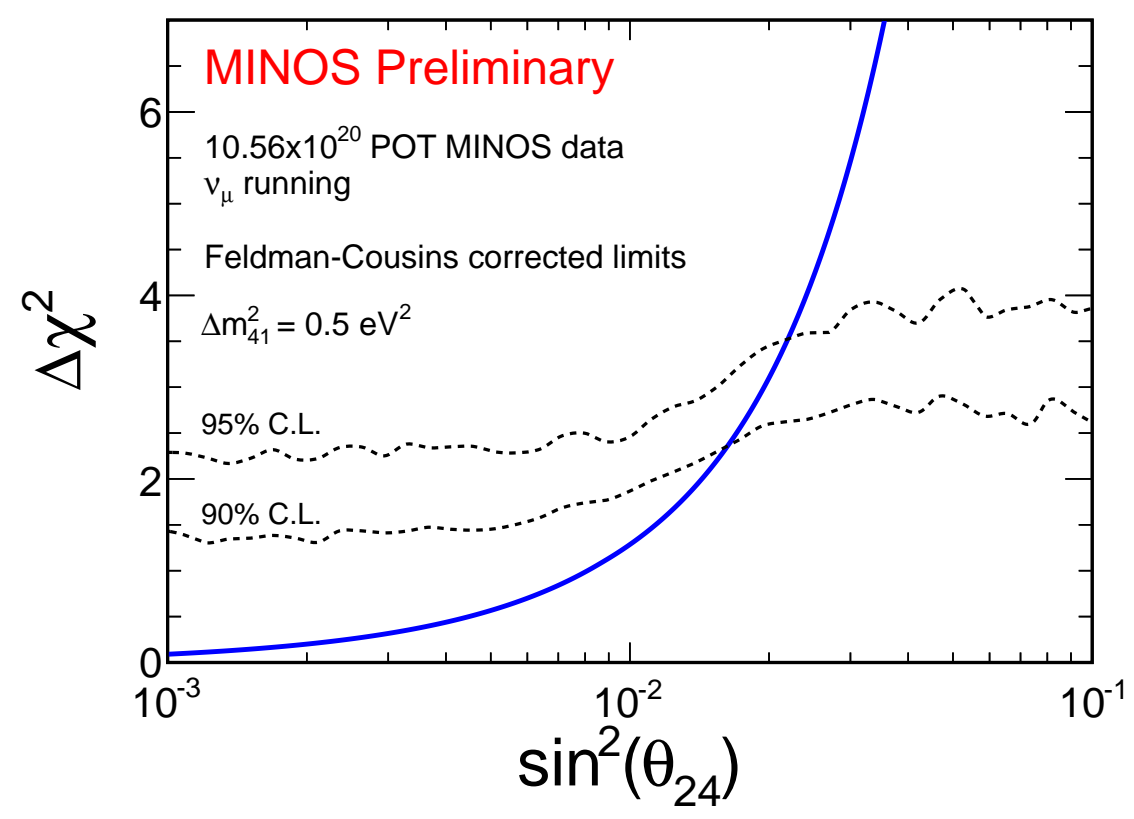

Figure 7.14: The $\chi^{2}$ surface for the sterile mixing angle $\theta_{24}$ for a fixed value of $\Delta m_{41}^{2}=0.5 \mathrm{eV}^{2}$. This limit is produced using the unified procedure of Feldman and Cousins and incorporates all sources of systematic uncertainty.

the disappearance channel. The MINOS and Super-Kamiokande results both use an exact $3+1$ oscillation model where Super-Kamiokande includes matter effects (necessary because of the much longer baseline in Super-Kamiokande) and MINOS takes the vacuum approximation. The other results assumed a large $\Delta m_{41}^{2}$ and therefore applied a simplified two-flavour approximation which has no sensitivity to the octant of $\theta_{24}$; therefore in order to display the experimental limits on an $x$-axis of $\sin ^{2} \theta_{24}$ these limits have been made symmetric about $\sin ^{2} \theta_{24}=0.5$ $\left(\theta_{24}=\pi / 4\right)$.

\subsubsection{Sterile Mixing Angle Limits with a Fixed $\Delta m_{41}^{2}$}

The sterile mixing angles and the sensitivity one has to them is determined by the value of $\Delta m_{41}^{2}$. Figures 7.14 and 7.15 place limits on the mixing angles $\theta_{24}$ and $\theta_{34}$ respectively by fixing the value of the mass splitting. The value of $\Delta m_{41}^{2}=0.5 \mathrm{eV}^{2}$ is chosen due to this being in the fast-oscillation region and in a 


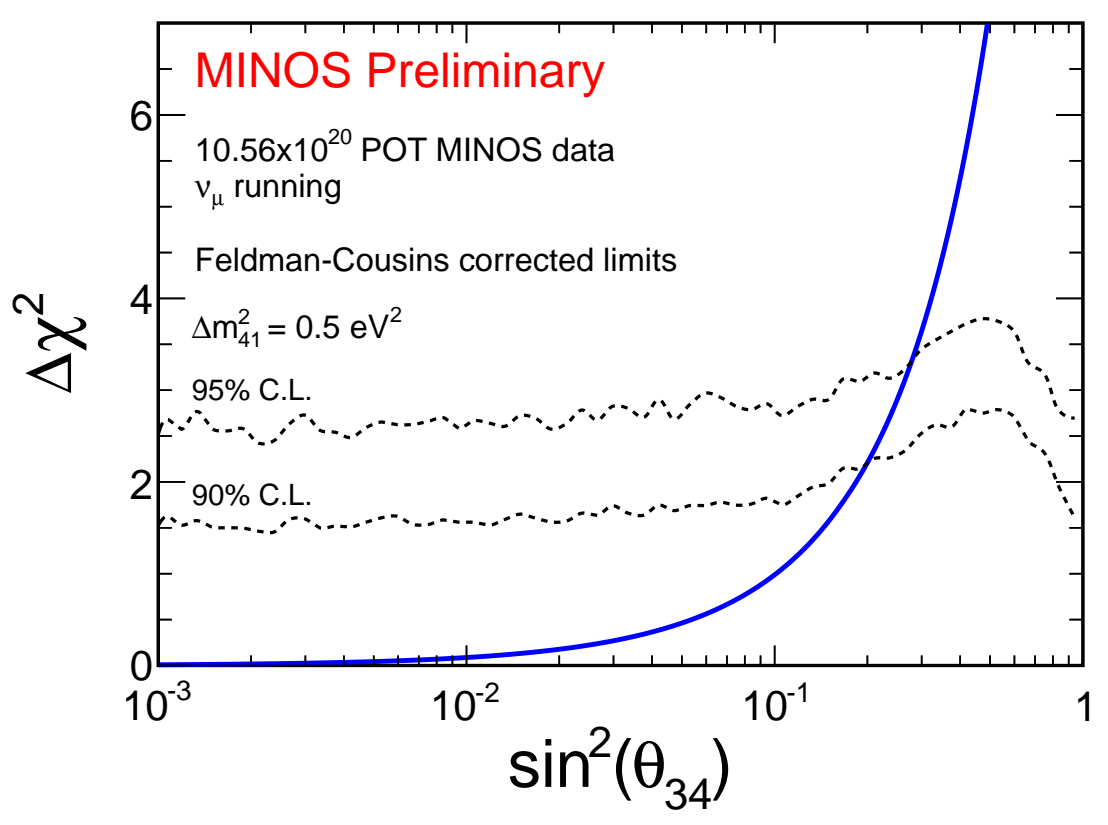

Figure 7.15: The $\chi^{2}$ surface for the sterile mixing angle $\theta_{34}$ for a fixed value of $\Delta m_{41}^{2}=0.5 \mathrm{eV}^{2}$. This limit is produced using the unified procedure of Feldman and Cousins and incorporates all sources of systematic uncertainty.

region where no oscillations occur at the MINOS ND so that the angle limits do not depend strongly on the mass splitting.

To produce the $1 \mathrm{D} \chi^{2}$ distributions for the sterile angles $\theta_{24}$ and $\theta_{34}$ a fit is performed that is similar to that which yielded the likelihood surface in figure 7.10. When fitting for $\theta_{24}$ the angle $\theta_{34}$ is allowed to vary. When fitting for $\theta_{34}$ the angle $\theta_{24}$ is allowed to vary. The confidence limits produced in figures 7.14 and 7.15 were created using the Feldman-Cousins method in a similar way to that of the production of the $2 \mathrm{D}$ confidence limits shown in figure 7.13 . This is explained in chapter 8 .

The upper limits placed on the mixing angles are: 


$$
\begin{aligned}
& \left|U_{\mu 4}\right|^{2}<\sin ^{2} \theta_{24}<0.01690 \% \text { C.L. } \\
& \left|U_{\mu 4}\right|^{2}<\sin ^{2} \theta_{24}<0.02295 \% \text { C.L. } \\
& \left|U_{\tau 4}\right|^{2}<\sin ^{2} \theta_{34}<0.20090 \% \text { C.L. } \\
& \left|U_{\tau 4}\right|^{2}<\sin ^{2} \theta_{34}<0.28090 \% \text { C.L. }
\end{aligned}
$$

\subsection{Effect of Systematic Uncertainties}

Figure 7.16 shows the effect on the $90 \%$ sensitivity C.L. of individually adding each systematic covariance matrix to the fit. Only the largest and most dominant systematics are included individually in comparison to the statistics only and total systematics contours. The largest systematics are labelled as Normalisation, Acceptance, and NC selection, with all other systematic uncertainties grouped as "other". For clarity, these systematic contours are related to the systematics discussed in chapter 6 in the following way. The contour labelled Normalisation refers to the relative normalisation systematic (section 6.2.1). The Acceptance contour refers to the acceptance and selection efficiency in the ND uncertainty (section 6.2.2) and the NC selection contour refers to the poorly-reconstructed events uncertainty (section 6.2.4).

The effect of cumulatively adding the systematic uncertainties together is shown in figure 7.17. For more information on these systematic uncertainties and their sizes see chapter 6. Both figures 7.16 and 7.17 contain confidence limits constructed using the Feldman-Cousins unified approach. 


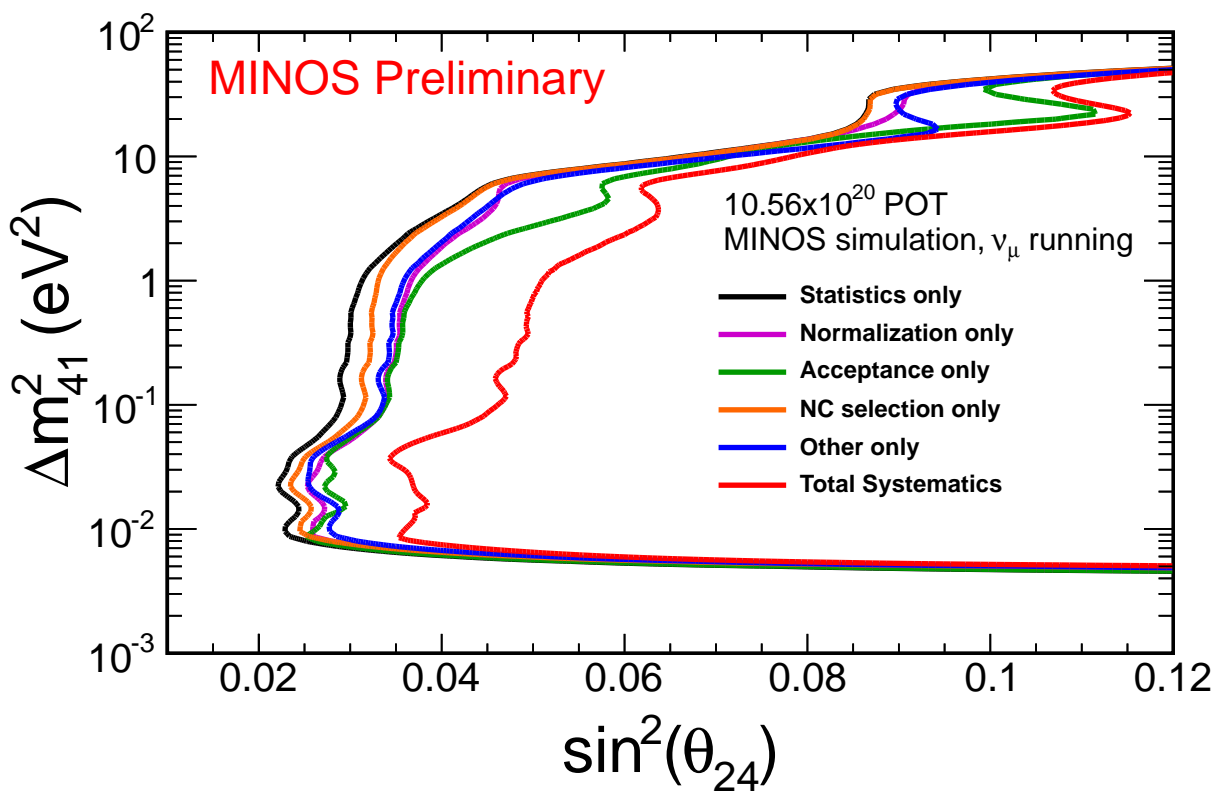

Figure 7.16: Individual effect of the sources of systematic uncertainty on the $90 \%$ C.L. sensitivity with the Feldman Cousins unified approach being used.

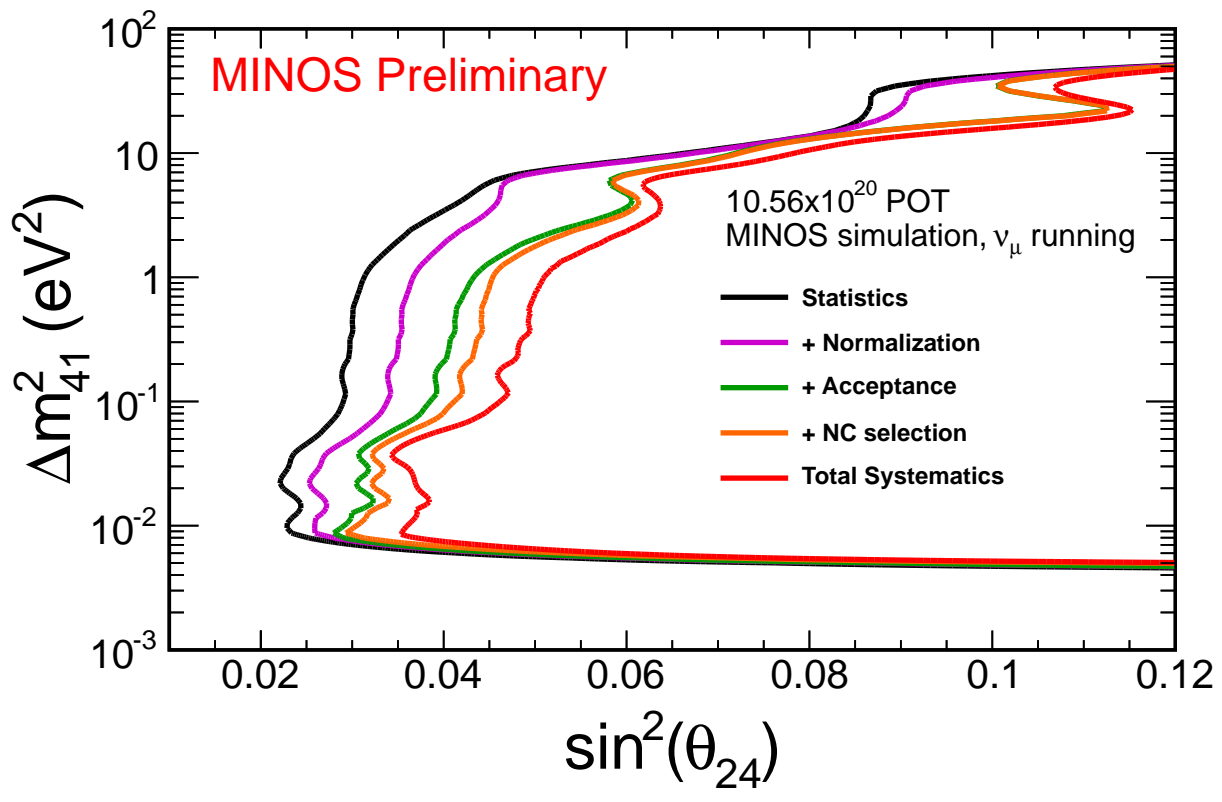

Figure 7.17: The effect of the largest systematic uncertainties used in the construction of the covariance matrix and how they affect the sensitivity of the $90 \%$ C.L. contour for MINOS. Each source of systematic uncertainity is added incrementally, finally obtaining the total systematic line. These contours have been constructed using the Feldman-Cousins procedure. 


\subsection{Combination with the Bugey-3 Reactor Ex- periment}

Appendix A shows that, in an experiment looking at $\nu_{e} / \bar{\nu}_{e}$ appearance in the limit of a high mass splitting such that $\Delta m_{41}^{2} \simeq \Delta m_{42}^{2} \simeq \Delta m_{43}^{2} \gg\left|\Delta m_{32}^{2}\right| \gg \Delta m_{21}^{2}$, the electron neutrino appearance probability from a muon neutrino beam can be approximated to

$$
\begin{aligned}
P_{\nu_{\mu} \rightarrow \nu_{e}} & \approx 4\left|U_{\mu 4}\right|^{2}\left|U_{e 4}\right|^{2} \sin ^{2}\left(\frac{\Delta m_{41}^{2} L}{4 E}\right) \\
& \approx \sin ^{2} \theta_{\mu e} \sin ^{2}\left(\frac{\Delta m_{41}^{2} L}{4 E}\right) .
\end{aligned}
$$

Therefore the experimental results from LSND and MiniBooNE can be interpreted as the magnitude of the mixing being governed by an effective mixing angle $\theta_{\mu e}$ that depends on the matrix elements $\left|U_{\mu 4}\right|^{2}$ and $\left|U_{e 4}\right|^{2}$. In terms of mixing angles this effective sterile mixing angle can be expressed as

$$
\sin ^{2} 2 \theta_{\mu e}=\sin ^{2} 2 \theta_{14} \sin ^{2} \theta_{24}
$$

As explained in the previous sections of this chapter, the MINOS experiment looks at muon neutrino survival and is sensitive primarily to the sterile mixing angle $\theta_{24}$. Throughout the analysis the assumption that $\theta_{14}=0$ is taken and therefore by placing limits on the mixing angle $\theta_{24}$ MINOS is placing constraints on the matrix element $\left|U_{\mu 4}\right|^{2}$. A reactor experiment such as Bugey-3 [175] looking at electron anti-neutrino disappearance from an electron anti-neutrino source will be analogous to the MINOS case and will have sensitivity primarily to $\theta_{14}$, and therefore the matrix element $\left|U_{e 4}\right|^{2}$ as shown in figure 7.18. Thus by combining the data from MINOS $\nu_{\mu}$-disappearance search with data from the Bugey-3 reactor $\bar{\nu}_{e}$-disappearance search [176], a direct comparison can be made to the LSND and 


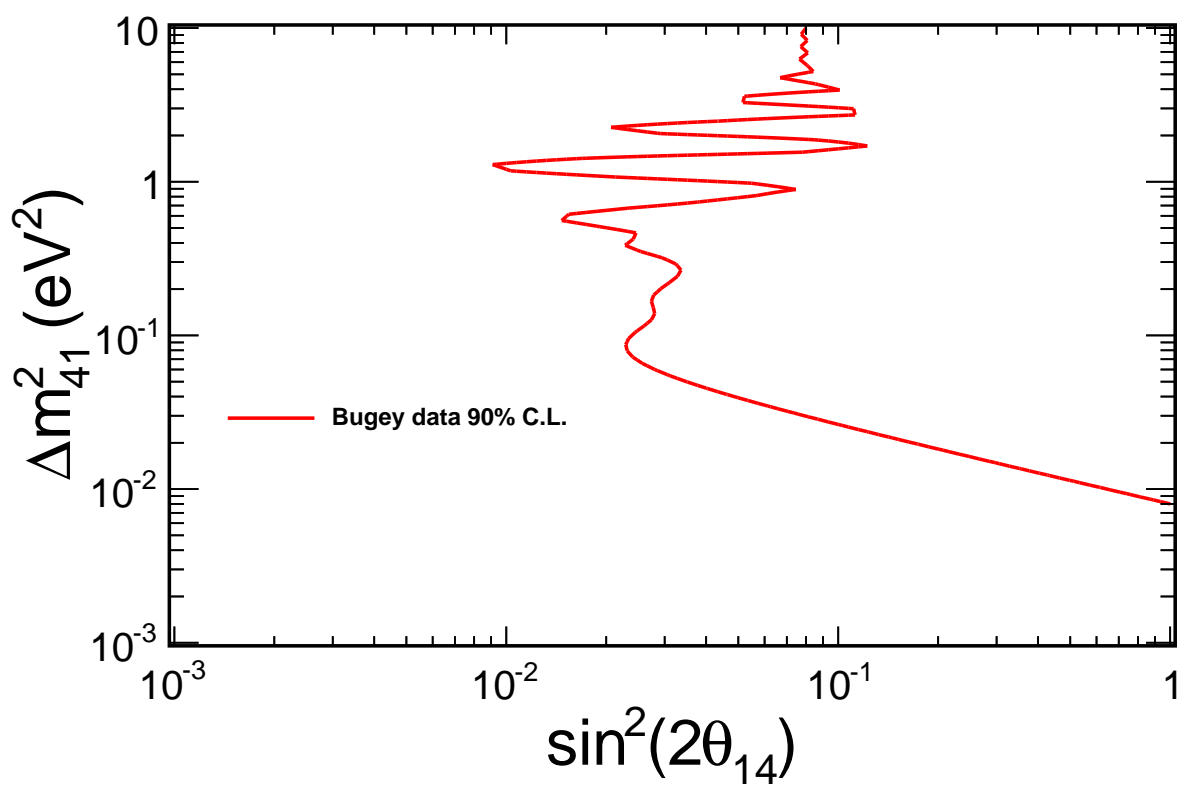

Figure 7.18: Bugey 90\% C.L. exclusion contour for the sterile mixing parameter $\sin ^{2}\left(2 \theta_{14}\right)$ by looking at electron anti-neutrino disappearance.

MiniBooNE appearance anomalies through the effective mixing angle $\theta_{\mu e}$.

Figure 7.19 shows the $90 \%$ C.L. from the combination between the MINOS and Bugey-3 experiments compared to the LSND [63] electron anti-neutrino appearance search, and several electron neutrino appearance searches: MiniBooNE [64], OPERA [177], ICARUS [74], and NOMAD [178].

The Bugey-3 experiment performed a measurement of the anti-electron neutrino energy spectrum using modules loaded with Lithium-6 scintillator at 15, 40 and 95 metres from the Bugey 2800 Megawatt reactor in France. To perform the combination, the Bugey-3 surface in the $\left\{\Delta m_{41}^{2} \sin ^{2} 2 \theta_{14}\right\}$ plane was obtained from the limit evaluated by Patrick Huber using the GLoBES software, incorporating the updated calculation of the reactor fluxes described in [179].

The combined limit is calculated by iterating through every value of $\Delta m_{41}^{2}$ and multiplying the correct combination of angles to obtain the effective mixing angle shown in equation 7.22. The corresponding $\chi^{2}$ value from that bin is summed from both the MINOS and Bugey surfaces. There are multiple combinations 


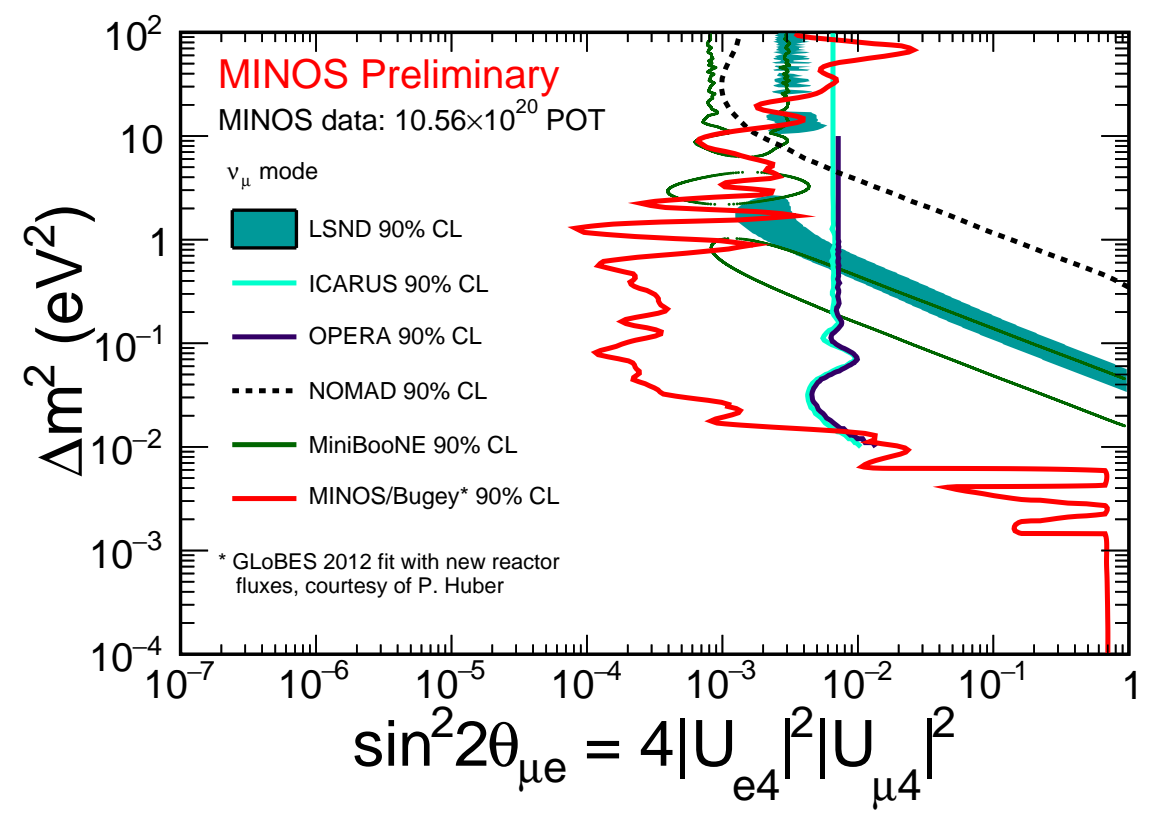

Figure 7.19: MINOS and Bugey-3 combined 90\% C.L. limit on the sterile mixing parameter $\sin ^{2} 2 \theta_{\mu e}=4\left|U_{e 4}\right|^{2}\left|U_{\mu 4}\right|^{2}$, obtained from the individual disappearance limits of each experiment on $\left|U_{\mu 4}\right|^{2}$ and $\left|U_{e 4}\right|^{2}$, respectively. Regions of parameter space to the right of the red contour are excluded at $90 \%$ C.L. The MINOS limits correspond to a $10.56 \times 10^{20} \mathrm{POT}$ exposure in neutrino running mode. The Bugey limits are computed accounting for the new calculation of reactor fluxes [179]. 
of $\sin ^{2} \theta_{24}$ and $\sin ^{2} 2 \theta_{14}$ that yield any particular value of $\sin ^{2} 2 \theta_{\mu e}$, therefore the combination with the lowest $\chi^{2}$ is taken to be the value for the combined likelihood surface.

This chapter has looked for $\nu_{\mu}$ disappearance and a deficit of NC events by performing a fit to the $\mathrm{F} / \mathrm{N}$ ratios for both the $\mathrm{CC}$ and $\mathrm{NC}$ samples. This analysis has set stringent limits on the existence of sterile neutrinos using the $3+1$ sterile neutrino model covering over five order of magnitude in $\Delta m_{41}^{2}$ as shown in figure 7.13. Figure 7.19 shows that in a combination with Bugey-3, a significant portion of the parameter space allowed by the LSND and MiniBooNE experiment has been rules out. 


\section{Chapter 8}

\section{Feldman-Cousins Confidence}

\section{Intervals}

This chapter discusses the process of constructing confidence limits in particle physics and the reason why they can become invalid for an analysis such as the one presented in this thesis. A study is performed to assess if the MINOS sterile neutrino analysis requires confidence intervals constructed using the Feldman Cousins unified approach. A detailed method of obtaining such confidence intervals is described.

\subsection{Introduction}

The Feldman-Cousins (FC) method [170] is frequentist in design. It allows an experiment to "calibrate" its actual statistical sensitivity at each point within a parameter space such that exact coverage is ensured when constructing a confidence limit (C.L.). This technique is one of brute force; by simulating both systematic and statistical fluctuations bin by bin to create an ensemble of fake data, the analysis framework is run using the thousands of fake experiments to manually calculate the degree of confidence that the truth lies within a confidence region in the parameter space of interest. 
A FC study is usually advised when the coverage of an experimental C.L. is suspected to be inaccurate (incorrect coverage) due to:

1. Limit construction under the assumption of Gaussian approximations within an analysis (Global and Raster scans, discussed later).

2. The treatment of best-fit points at an unphysical value.

3. Based on the data set measured, the user making the choice to give an upper limit on a null result or a two-sided confidence interval for a non-null result.

4. In neutrino physics the probability functions being sinusoidal. The $\chi^{2}$ distribution assumes a Gaussian probability density function, so when probing many orders of $\Delta m^{2}$ one can become susceptible to finding local and global minima due to fluctuations, the significances of which are highly non-Gaussian.

The FC method is designed to solve problems with constructing a C.L. in the cases described above. This credible method is a desirable approach when constructing confidence intervals, however the interpretations can be non-trivial and the procedure can be very CPU intensive often making it impractical.

\subsection{Some Statistical Theory}

\subsubsection{It began with Neyman the Frequentist}

Traditionally, high-energy particle physics experiments have taken the classical confidence interval approach. These intervals can be obtained by using what is known as the Neyman construction [180]. A classical confidence interval under this construction has a statement of the form: The unknown parameter $\theta$ lies 
within the region $\left[\theta_{1}, \theta_{2}\right]$ at a given confidence $\alpha$. This can be expressed mathematically [170] as

$$
P\left(\theta \in\left[\theta_{1}, \theta_{2}\right]\right)=\alpha
$$

where $\theta_{1}$ and $\theta_{2}$ are functions of the data set $x$. This is not a statement about the true value $\theta_{t}$ but more of a statement about the confidence intervals $\theta_{1}$ and $\theta_{2}$. For each value of $\theta$ one can calculate $P(x \mid \theta)$, and from this one selects the interval $\left[x_{1}, x_{2}\right]$ such that

$$
P\left(x \in\left[x_{1}, x_{2}\right] \mid \theta\right)=\alpha .
$$

As an example, picture an experiment with a measured dataset $x_{0}$. One can then obtain the lower limit $\theta_{1}$ and upper limit $\theta_{2}$ from calculating the probability of your dataset for a given $\theta$. By taking this frequentist approach one is hypothetically assuming a large ensemble experiments, in where each experiment will construct a different limit using an identical analysis framework but different observed dataset. One can then make the statement the unknown true value $\theta_{t}$ will be within a fraction $\alpha$ of the experiments' confidence limits. A strong emphasis is stressed to the reader that this is NOT the same as the true value $\theta_{t}$ has a probability $\alpha$ of being within an experiment's confidence limits. The main point from a frequentist point of view is that the unknown true parameter $\theta_{t}$ is at a fixed value; it is the shape of the C.L. that will vary due to statistical and systematic fluctuations between experiments, not the position of the true value $\theta_{t}$.

\subsubsection{Why not take a Bayesian Approach?}

Bayesian approaches are becoming more popular. The inferences made from a Bayesian credible interval are considered to be how many people think; people are hardwired to construct questions in a Bayesian manner. It can be intuitive 
for a person to think about the probability of some true value $\theta_{t}$ and how likely this is given a fixed dataset $x_{0}$, unlike a frequentist who considers $P\left(\theta_{t}\right)=1$ or 0 (a fixed value). An experiment will usually produce a likelihood surface, therefore a Bayesian approach makes inferences using a posterior probability density function (pdf) given as

$$
P\left(\theta_{t} \mid x_{0}\right)=P\left(x_{0} \mid \theta_{t}\right) P\left(\theta_{t}\right) / P\left(x_{0}\right) \text {. }
$$

The function $P\left(\theta_{t}\right)$ is known as the prior, and is the degree of belief in the true parameter $\theta_{t}$. This prior is based on theory and can be based on past experimental data and is often referred to as a subjective prior. A Bayesian approach is appealing in situations where the frequentist approach gives confusing results; such an example occurs when a best-fit point is calculated at a boundary (an artificial constraint implemented by the user) or within an unphysical region (e.g. $\sin (\theta)>1$ ). A Bayesian approach is not brute force and so by assuming the dataset $x_{0}$ is fixed there is no requirement for many thousands of fake experiments, unlike the Neyman construction.

\subsection{Global and Raster Scans}

Some argue that an experimental result should be completely independent of past measurements (or as independent as one can get). Therefore a frequentist approach can be desirable. However, frequentist limits via the Neyman construction can be CPU intensive and time consuming. There are several approximations one can make in an analysis such that the fundamentals of statistical analysis assuming Gaussian statistics hold true (most of the time); this allows for a more graphical method of constructing confidence intervals known as Global and Raster scans.

Global and Raster scan methods are traditionally based on log-likelihood ra- 
tios. The general approach is to create a likelihood surface $L(\boldsymbol{\theta} \mid \mathbf{x})$ that quantifies how likely it is that the observed data, $\mathbf{x}$, describes the theory given by a set of underlying parameters $\boldsymbol{\theta}=\left(\theta_{1}, \ldots, \theta_{N}\right)$ with some unknown true values. The value of $\boldsymbol{\theta}$ that is the most probable (most likely) value is expressed as $\boldsymbol{\theta}=\hat{\boldsymbol{\theta}}$, such that $L(\hat{\boldsymbol{\theta}})=L_{\mathrm{Max}}$, otherwise known as having the maximum likelihood. The likelihood is usually expressed as $-2 \ln L$; the function is monatomic, meaning its first derivative does not change sign, so the logarithmic operation does not affect the statistical interpretation of the likelihood. However, due to the minus sign the function now needs to minimised to find the most probable value of the prediction $\boldsymbol{\mu}(\boldsymbol{\theta})$ where $\boldsymbol{\mu}$ depends on the underlying set of parameters $\boldsymbol{\theta}$. An expression for the log-likelihood for binned data is given as

$$
-2 \ln L=2 \sum_{i=1}^{N}\left[\mu_{i}(\boldsymbol{\theta})-x_{i}+x_{i} \ln \frac{x_{i}}{\mu_{i}(\boldsymbol{\theta})}\right]+\sum_{j=1}^{M} \frac{\epsilon_{j}^{2}}{\sigma_{j}^{2}},
$$

where $\mu_{i}(\boldsymbol{\theta})$ is the prediction and $x_{i}$ is the data from the $i^{\text {th }}$ bin. The final term includes the $M$ systematic uncertainties, $j$, and treats them as nuisance parameters with values $\epsilon_{j}$ and uncertainties $\sigma_{j}$ whilst minimising the likelihood. One should note that the penalty term derives from Bayesian statistics; by adding a penalty term one is providing a prior pdf for the distribution of that systematic variable.

As $\mu_{i}$ becomes large the likelihood distribution converges onto a $\chi^{2}$ distribution as seen in equation 8.5,

$$
\chi^{2}=(\mathbf{x}-\boldsymbol{\mu}(\boldsymbol{\theta}))^{T} V^{-1}(\mathbf{x}-\boldsymbol{\mu}(\boldsymbol{\theta})),
$$

where the observed measurement is $\mathbf{x}$ and the expected value from simulation is $\boldsymbol{\mu}$, with $V^{-1}$ being the inverse of the covariance matrix. The likelihood and $\chi^{2}$ quantities can be related by $L \propto e^{-\chi^{2} / 2}$.

Both $-2 \ln L$ and $\chi^{2}$ are minimised by finding a set of parameters, $\boldsymbol{\theta}=\hat{\boldsymbol{\theta}}$, 
such that the first order derivative of the $\chi^{2}$ or log-likelihood is zero (a stationary point). A Global (Raster) scan is a graphical representation of the construction of confidence limits and assumes the $\chi^{2}$ and log-likelihood approximate to a quadratic around the minimum point $\boldsymbol{\theta}=\hat{\boldsymbol{\theta}}$. This becomes clear if one performs a Taylor expansion of the likelihood surface around the minimum point $\hat{\theta}$ for a one dimensional $\ln [L(\theta)]$ distribution:

$$
\ln L[(\theta)]=\ln [L(\hat{\theta})]+\left[\frac{\partial \ln L}{\partial \theta}\right]_{\theta=\hat{\theta}}(\theta-\hat{\theta})+\frac{1}{2 !}\left[\frac{\partial^{2} \ln L}{\partial \theta^{2}}\right]_{\theta=\hat{\theta}}(\theta-\hat{\theta})^{2}+\ldots
$$

It is known that at the point $\theta=\hat{\theta}, \ln L(\hat{\theta})=\ln L_{\max }$ and therefore the second term in equation 8.6 is at a stationary point and must be zero. If one assumes that, in this example, $\theta$ follows a Gaussian distribution, the second derivative term can be expressed as the uncertainty on the parameter $\theta$ as shown in equation 8.7:

$$
-\frac{1}{{\hat{\sigma^{2}}}_{\hat{\theta}}}=\left[\frac{\partial^{2} \ln L}{\partial \theta^{2}}\right]_{\theta=\hat{\theta}}
$$

Equation 8.7 is only true in the Gaussian limit. Higher order terms in the Taylor expansion are ignored, although note that they are non-zero and become increasingly non-negligible as $\mu_{i} \rightarrow 0$, which is why for a low number of events a likelihood surface becomes non-symmetric around $\ln L_{\max }$.

Using the definition of equation 8.7 one can simplify equation 8.6 to the following:

$$
\ln L(\theta)=\ln L_{\max }-\frac{(\theta-\hat{\theta})^{2}}{2 \sigma_{\hat{\theta}}^{2}} .
$$

By inserting the parameter $\hat{\theta} \pm \hat{\sigma}_{\hat{\theta}}$ into equation 8.8, it then follows that

$$
\ln L\left(\hat{\theta} \pm \hat{\sigma}_{\hat{\theta}}\right)=\ln L_{\max }-\frac{1}{2}
$$

It is from equation 8.9 that one can see how this method makes use of the likelihood ratios. A change in the parameter $\theta$ by one standard deviation $\sigma$ from its 
maximum likelihood value equates to an increase in the likelihood by half a unit, which is an equivalent statement to saying that the probability decreases by $e^{-\frac{1}{2}}$. One can rewrite equation 8.9 in terms of a $\chi^{2}$ :

$$
-2 \ln L\left(\hat{\theta} \pm \hat{\sigma}_{\hat{\theta}}\right) \approx \chi^{2}\left(\hat{\theta} \pm \hat{\sigma}_{\hat{\theta}}\right) \approx \chi_{\min }^{2}+1
$$

This one dimensional example can be generalised for $\alpha$ parameters and expressed for $\xi$ number of sigma for a given confidence level (C.L.) as given by equation 8.11:

$$
\chi^{2}(\hat{\theta} \pm \xi \hat{\sigma})=\chi_{\min }^{2}+\chi_{\alpha}^{2}(\text { C.L. }) .
$$

Table 8.1 shows the possible values of $\chi_{\alpha}^{2}$ (C.L.) for the number of degrees of freedom, $\alpha$.

\begin{tabular}{ccccc}
\hline \hline$\chi_{\alpha}^{2}$ & $68 \%$ & $90 \%$ & $95 \%$ & $99 \%$ \\
\hline$\chi_{1}^{2}$ & 1.00 & 2.71 & 3.84 & 6.63 \\
$\chi_{2}^{2}$ & 2.28 & 4.61 & 5.99 & 9.21 \\
\hline
\end{tabular}

Table 8.1: $\chi^{2}$ quantile values for various confidence intervals for different numbers of degrees of freedom, $\alpha$.

The common approach is for an experiment to produce a confidence region using equation 8.11. However, one must take care since the approximations used to derive this expression are not always valid. The main problem is a question of how Gaussian the statistics of the analysis are. The central limit theorem [181] states that under certain conditions, the distribution of a parameter becomes Gaussian as $\mu_{i} \rightarrow \infty$, however this is not a guarantee.

Equation 8.6 shows that for a Global or Raster scan approach to creating confidence intervals to be valid the likelihood surface must be a parabola around the most likely value of the parameter of interest $\theta$. However, this does not have to be the case; the maximum likelihood estimator of $\theta$ is invariant, meaning that in- 
stead of estimating the parameter $\theta$ one can perform a transformation of variables and come to the same conclusions for the most likely value and confidence intervals [182]. Thus, a non-parabolic likelihood function $L(\theta)$, through a transformation of variables $\theta \rightarrow g(\theta)$, will result in a new likelihood surface with parabolic shape. One can then define a confidence interval in terms of $\left[g\left(\theta_{1}\right), g\left(\theta_{2}\right)\right]$ and by the invariance property $\ln L[\hat{\theta}]=\ln L[g(\hat{\theta})]$, obtain the confidence interval values for $\left[\theta_{1}, \theta_{2}\right]$. Therefore, due to this invariance property it is possible to make inferences about a parameter with a non-parabolic likelihood function without actually finding a transformation of variables to obtain a parabolic solution.

A Global scan is used on a two-dimensional surface by defining a confidence region by increasing the $\chi^{2}$ by $\chi_{2}^{2}$ (C.L.) around the most likely point. A Raster scan takes a more conservative approach and for a fixed value of one parameter finds the local minimum for the second. This method is considered the "least powerful" out of the two. Feldman and Cousins [170] extensively compare these two methods.

\subsection{Does the MINOS Sterile Neutrino Analysis need an FC correction?}

A mock-data study was conducted on the MINOS sterile neutrino analysis at an early stage. This mock data study was conducted by creating likelihood surfaces in the plane $\left\{\Delta m_{41}^{2}, \sin ^{2} \theta_{24}\right\}$ using equation 8.4 but only considering statistics (setting the final term to zero).

To test how suitable a Global or Raster scan approach would be many threeflavour FD fake-data predictions were created for both the CC and NC reconstructed neutrino energy spectra. Each fake data prediction was created under the assumption of the null-hypothesis (i.e. no sterile neutrinos); the contents of each bin were statistically fluctuated according to a Poisson distribution. For 


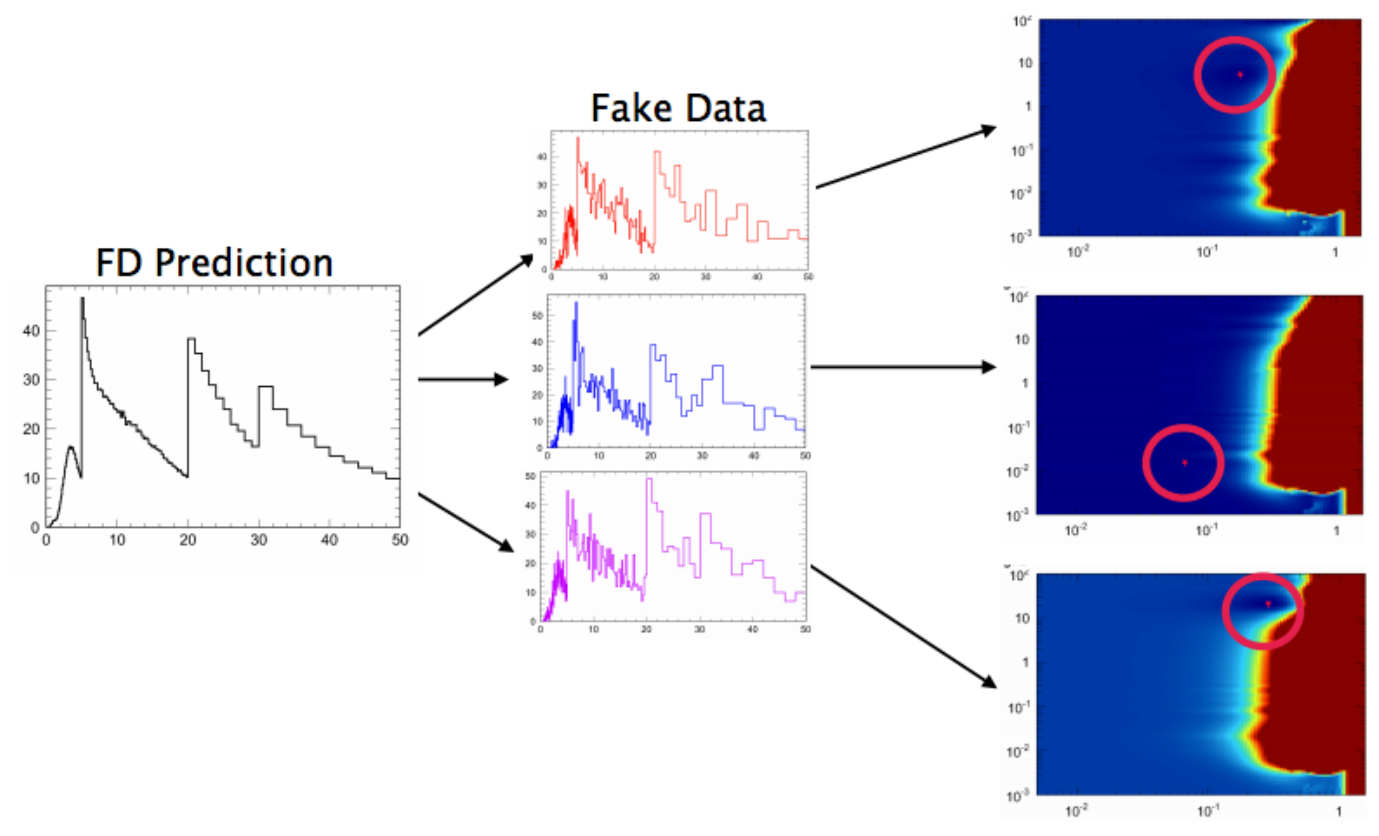

Figure 8.1: An example of a mock-data study with statistically fluctuated Far Detector fake data. We can see how the global minimum (pink star) moves around.

each three-flavour energy spectrum the MINOS sterile neutrino analysis framework was used to create a likelihood surface. A conceptual diagram is shown in figure 8.1.

For each surface the smallest $\chi^{2}$ value was defined as $\chi_{\text {bestfit. }}^{2}$ Each set of fake data was also compared to a three-flavour-only model (the null hypothesis, setting all sterile parameters to zero). The null-hypothesis $\chi^{2}$ value is defined as $\chi_{\text {null }}^{2}$. The difference between these two variables is defined as $\Delta \chi^{2}=\chi_{\text {bestfit }}^{2}-\chi_{\text {null }}^{2}$; the distribution of this test-statistic is shown in figure 8.2. Under Gaussian approximations one would expect this to follow a $\chi^{2}$ distribution due to Wilks' theorem [183].

Figure 8.2 shows the distribution of $\Delta \chi^{2}$ for 133 fake experiments (this study was CPU intensive). A global scan method is chosen and $90 \%$ confidence intervals were constructed using equation 8.11, with the values of $\chi_{\alpha}^{2}$ from table 8.1 for a two-degree-of-freedom parameter space (i.e. $\Delta \chi^{2}=4.61$ ). One can see that only 


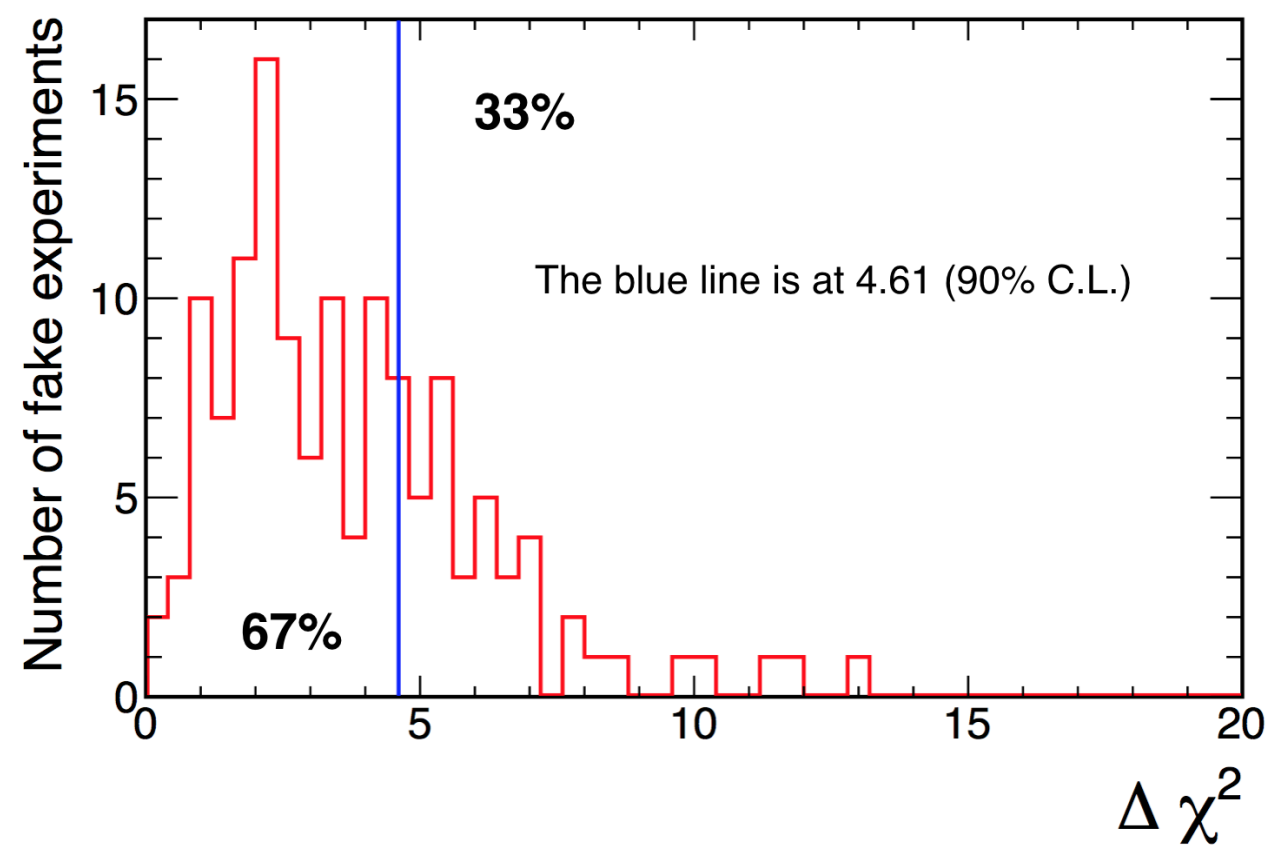

Figure 8.2: The $\Delta \chi^{2}$ distribution for 133 fake experiments. The blue line indicates the value of $\chi_{2}^{2}$ for constructing a $90 \%$ confidence limit on a two dimensional plane using the Global scan method. $67 \%$ of fake experiments have a $\Delta \chi^{2}$ to the left of the blue line, $33 \%$ have a $\Delta \chi^{2}$ to the right of the blue line. 
$67 \%$ of experiments have a 90\% C.L interval that includes the null hypothesis (the true hypothesis in this study). This implies that the Global scan approach for this analysis "under covers" the parameter space, implying that using the prescribed method of likelihood ratio confidence limit construction would yield incorrect confidence intervals for estimating the statistical error. This strongly suggests that a different method has to be used for creating confidence intervals.

\subsection{The Feldman-Cousins Procedure}

The proposed technique is one of brute force. At each point in the parameter space $\mathbf{T}$, a large number of fake experiments are produced. The fake data are generated with a signal at each point (i.e. values of $\Delta m_{41}^{2}$ and $\sin ^{2} \theta_{24}$ ) and are fluctuated using the covariance matrix used in the fit; both statistical and systematic fluctuations are applied to the data. For more details on this procedure see section 8.6.

Each point in the parameter space will have $N$ experiments. For each of the fake experiments, a test statistic, $R$, is defined as

$$
R=\frac{P\left(\mathbf{x} \mid \mathbf{T}, \boldsymbol{\xi}^{\text {best }}\right)}{P\left(\mathbf{x} \mid \mathbf{T}^{\text {best }}, \boldsymbol{\xi}^{\text {best }}\right)}
$$

where $\mathbf{x}$ is the simulated fake dataset for point $\mathbf{T}$ with nuisance parameters $\boldsymbol{\xi}$. The highest probability for $P\left(\mathbf{x} \mid \mathbf{T}, \boldsymbol{\xi}^{\text {best }}\right)$ is given by $\mathbf{T}^{\text {best }}$ and $\boldsymbol{\xi}^{\text {best }}$, where the superscript "best" implies the parameters have been allowed to vary within the fit to provide the highest probability for the simulated fake dataset $\mathbf{x}$.

Assuming the probability distribution is Gaussian, $\chi^{2}=-2 \ln (P)$. The test 
statistic $R$ is equivalent to using the difference in $\chi^{2}$ between $\mathbf{T}$ and $\mathbf{T}^{\text {best }}$ :

$$
\begin{aligned}
R^{\prime} \equiv \Delta \chi^{2} & =\chi^{2}\left(\mathbf{T}, \boldsymbol{\xi}^{\text {best }}\right)-\chi^{2}\left(\mathbf{T}^{\text {best }}, \boldsymbol{\xi}^{\text {best }}\right) \\
& =\chi_{\text {profile }}^{2}-\chi_{\text {best }}^{2} .
\end{aligned}
$$

At each point in the parameter space $\mathbf{T}$ one can obtain a distribution of $R^{\prime}$ over the $N$ fake experiments generated at $\mathbf{T}$. From this distribution one can determine the value $\Delta \chi_{c}^{2}$ such that $\alpha$ of the simulated experiments have $\Delta \chi^{2}<\Delta \chi_{c}^{2}$. One might naively expect that $\Delta \chi_{c}^{2}=4.61$ for the $90 \%$ C.L. values for a $\chi^{2}$ distribution with two degrees of freedom but this often not be the case. One can then perform the standard procedure of constructing a contour from the data by including points that satisfy $\Delta \chi_{\text {data }}^{2}<\Delta \chi_{c}^{2}$.

\subsubsection{Applying the Feldman-Cousins procedure to the Ster- ile Neutrino Analysis}

The MINOS analysis fits the $\mathrm{CC}$ and $\mathrm{NC} \mathrm{F} / \mathrm{N}$ ratios as functions of reconstructed energy. The analysis considers the parameter space $\mathbf{T}=\left\{\Delta m_{41}^{2}, \sin ^{2} \theta_{24}\right\}$ resulting in a likelihood surface with two degrees of freedom. The analysis has several nuisance parameters defined as $\boldsymbol{\xi}=\left\{\Delta m_{32}^{2}, \sin ^{2} \theta_{23}, \sin ^{2} \theta_{34}\right\}$; their values are profiled during the fit. No systematic uncertainties are fit directly, instead they are incorporated into a covariance matrix. The fit procedure is identical to the analysis presented in chapter 7 .

At each point on the plane $\mathbf{T}=\left\{\Delta m_{41}^{2}, \sin ^{2} \theta_{24}\right\}, N=4000$ fake experiments were produced for both the $\mathrm{CC}$ and $\mathrm{NC} \mathrm{F/N}$ ratios. Each fake experiment was produced with sterile parameters $\Delta m_{41}^{2}$ and $\theta_{24}$ at the point $\mathbf{T}$ with the nuisance parameters $\theta_{23}$ and $\theta_{34}$ chosen as the best fits to data at the point T. MINOS has limited sensitivity to the octant of $\theta_{23}$, meaning it is not necessary to create an equal proportion of fake experiments for both octants of $\theta_{23}$. 
At every point $\mathbf{T}$ an equal number of fake data sets is computed for both the normal and inverted mass ordering of $\Delta m_{32}^{2}$. The absolute value of $\Delta m_{32}^{2}$ is taken as the best fit to the real dataset used in the fit in chapter 7 and then the sign is reversed for half of the experiments. The MINOS analysis profiles over both the normal and inverted mass hierarchies of $\Delta m_{32}^{2}$ within the fit.

Varying the nuisance parameter values for the fake data experiments is technically a more accurate representation of generating fake data. However, this requires the user to provide priors for these variables. By keeping the values fixed one can avoid having to justify priors, which is of particular of concern for the value of $\theta_{34}$. A study was performed at an arbitrary point $\mathbf{T}$, where the nuisance parameters $\Delta m_{32}^{2}$ and $\theta_{23}$ (belonging to $\boldsymbol{\xi}$ ) were represented by Gaussian priors with mean values

$$
\begin{aligned}
{\overline{\Delta m_{32}}}_{32}^{2} & =2.41 \times 10^{-3} \mathrm{eV}^{2} \text { (normal ordering) } \\
\bar{\theta}_{23} & =0.695 \text { (lower octant) } .
\end{aligned}
$$

These values were taken from [79]. The widths of the Gaussian priors were intentionally large, in both cases $2 \sigma$ of the errors calculated in [79]. For the same point $\mathbf{T}$ this procedure was also performed with fake data generated at fixed values of $\Delta m_{32}^{2}$ and $\theta_{23}$ using the mean values quoted above. The outcome of the test statistic $R^{\prime}$ was not significantly different whether the values of the nuisance parameters were fixed or allowed to vary. The MINOS sterile neutrino analysis is least sensitive to the nuisance parameter $\theta_{34}$, therefore if the nuisance parameters $\Delta m_{32}^{2}$ and $\theta_{23}$ can be kept fixed one can justify keeping $\theta_{34}$ fixed. The rest of this chapter will address the creation of the Feldman-Cousins confidence intervals where all $N$ experiments at a point $\mathbf{T}$ use fixed values of $\boldsymbol{\xi}$ for the creation of fake data, with values obtained from the best fit to the MINOS data.

The test statistic $R^{\prime}$ is evaluated at each computed point $\mathbf{T}$, according to 
equation 8.13. An example of two $R^{\prime}$ distributions are presented in figures 8.3 and 8.4 for determining the $\Delta \chi_{c}^{2}$ value for a $90 \%$ C.L. for two degrees of freedom, along with the best fit distributions of the nuisance parameters $\boldsymbol{\xi}^{\text {best }}$.

Figure 8.3 is calculated using the true values $\Delta m_{41}^{2}=4 \mathrm{eV}^{2}, \theta_{24}=0.32$, $\theta_{23}=0.78, \theta_{34}=0.4$ and $\left|\Delta m_{32}^{2}\right|=0.00232 \mathrm{eV}^{2}$, which are values obtained from the fit to the real MINOS data at this point $\mathbf{T}$. For this example, $\Delta \chi_{c}^{2}=$ 4.63, which is close to the value one would assume if using a Global scan in a region where Gaussian statistics holds true (4.61). The $\Delta \chi^{2}$ looks like a typical $\chi^{2}$ distribution for two degrees of freedom and the best fit parameters have a Gaussian distribution around the true values, except $\theta_{23}$ and $\Delta m_{32}^{2}$ due to the ambiguities of the mass ordering and octant of $\theta_{23}$.

Figure 8.4 is calculated using the true values $\Delta m_{41}^{2}=0.03 \mathrm{eV}^{2}, \theta_{24}=0.18$, $\theta_{23}=0.78, \theta_{34}=0.4$ and $\left|\Delta m_{32}^{2}\right|=0.00232 \mathrm{eV}^{2}$, which are values obtained from the fit to the real MINOS data at this point $\mathbf{T}$. For this example, $\Delta \chi_{c}^{2}=6.32$, which is significantly different from the assumption of Gaussian statistics. The $\Delta \chi^{2}$ follows a $\chi^{2}$ distribution of more than two degrees of freedom and the best fit distribution for $\Delta m_{41}^{2}$ in particular is not a Gaussian distribution. This is an example of the Feldman-Cousins unified approach adjusting the confidence interval compared to the traditional method of using a Global scan. To ensure exact coverage the value of $\Delta \chi_{c}^{2}$ has been significantly increased. The main driver of this is the sinusoidal nature of the neutrino oscillations probabilities, such that multiple values of $\Delta m_{41}^{2}$ give a good fit to the statistical fluctuations in the data due to such a large range of values being probed.

Ideally the test statistic $R^{\prime}$ would be computed at each point in the parameter space T. Due to CPU constraints and time this brute force method could not realistically achieve this. However, it is only necessary to know $R^{\prime}$ at points in the parameter space $\mathbf{T}$ where the confidence intervals are likely to fall. Although the Global scan method does not provide good coverage for this analysis is can 

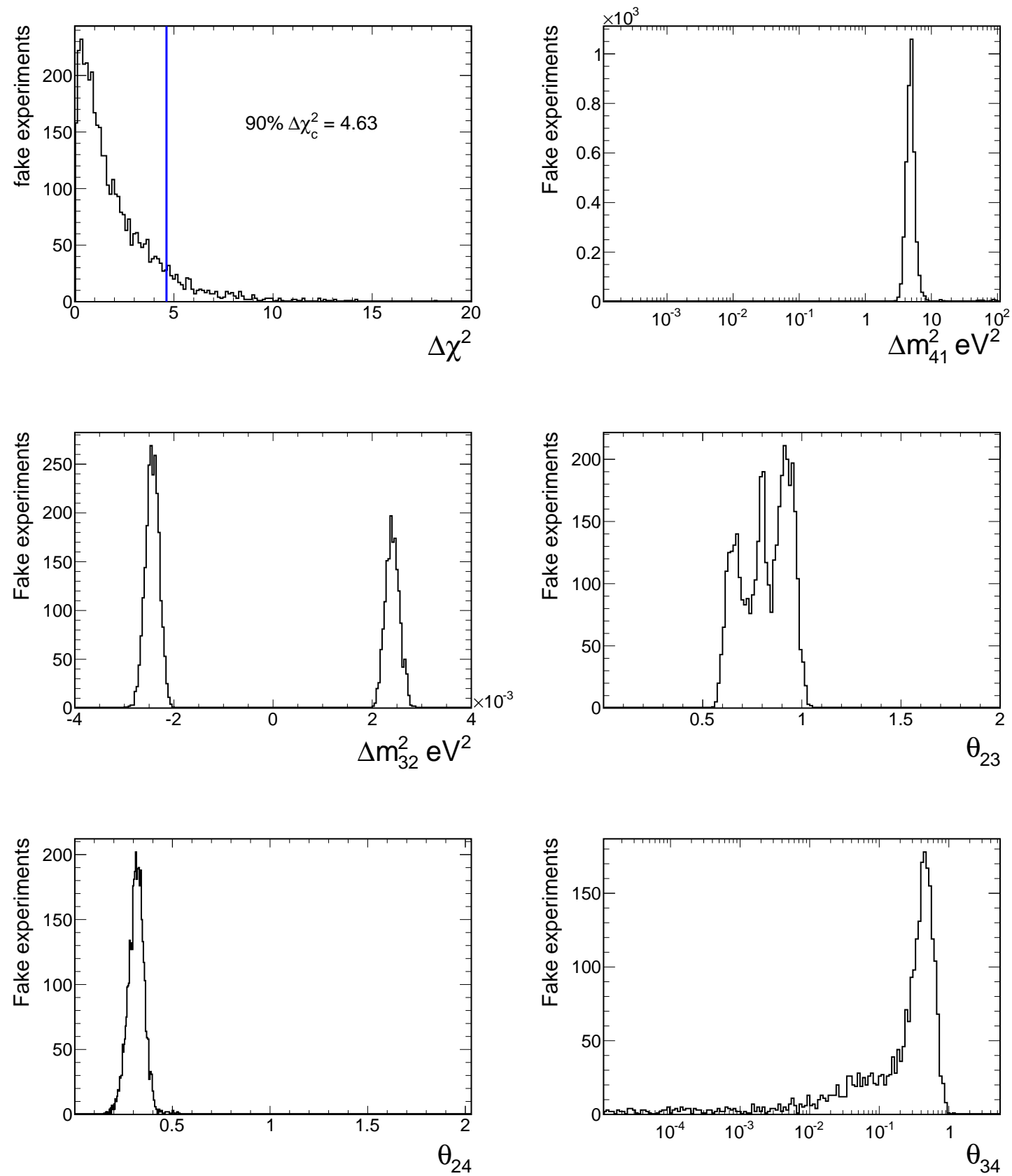

Figure 8.3: The $\Delta \chi^{2}$ distribution at a point $\mathbf{T}$ for $N=4000$ fake experiments using the underlying true values of $\Delta m_{41}^{2}=4 \mathrm{eV}^{2}, \theta_{24}=0.32, \theta_{23}=0.78, \theta_{34}=0.4$ and $\left|\Delta m_{32}^{2}\right|=0.00232 \mathrm{eV}^{2}$. The best fit distributions of the nuisance parameters $\boldsymbol{\xi}^{\text {best }}$ are also shown. 

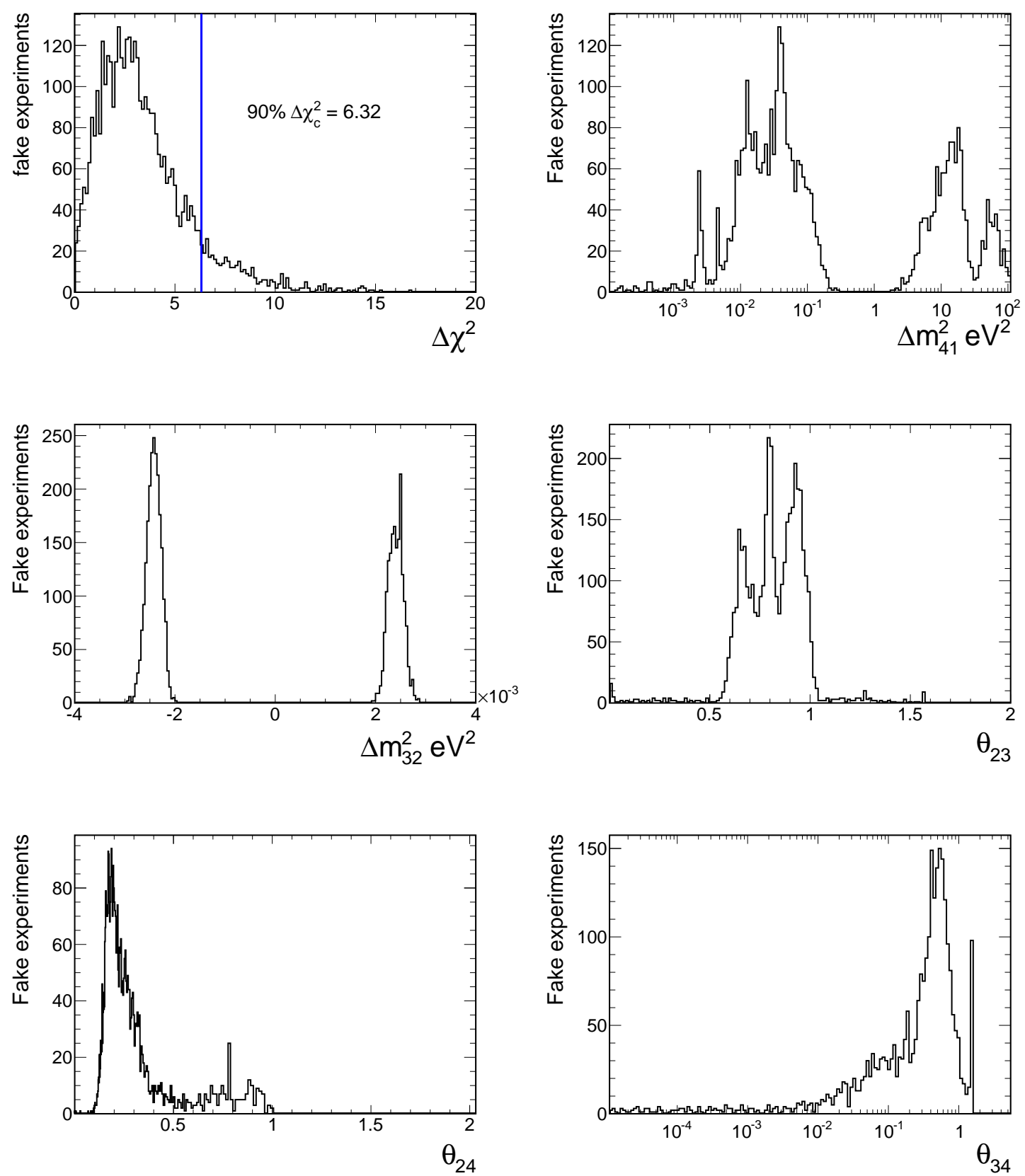

Figure 8.4: The $\Delta \chi^{2}$ distribution at a point $T$ for $N=4000$ fake experiments using the underlying true values of $\Delta m_{41}^{2}=0.03 \mathrm{eV}^{2}, \theta_{24}=0.18, \theta_{23}=0.78$, $\theta_{34}=0.4$ and $\left|\Delta m_{32}^{2}\right|=0.00232 \mathrm{eV}^{2}$. The best fit distributions of the nuisance parameters $\boldsymbol{\xi}^{\text {best }}$ are also shown. 
be used to find the approximate regions of the required confidence interval. This is then refined using the Feldman-Cousins procedure until the final confidence limits have been located. Figure 8.5 shows the $R^{\prime}$ test statistic calculated in the regions of $\left\{\Delta m_{41}^{2}, \sin ^{2} \theta_{24}\right\}$ parameter space necessary for calculating the $90 \%$ C.L. contour. The blue regions shown in figure 8.5 have a $\Delta \chi_{c}^{2}$ value similar to that for Gaussian statistics. All other regions require the $\Delta \chi_{c}^{2}$ to be modified.

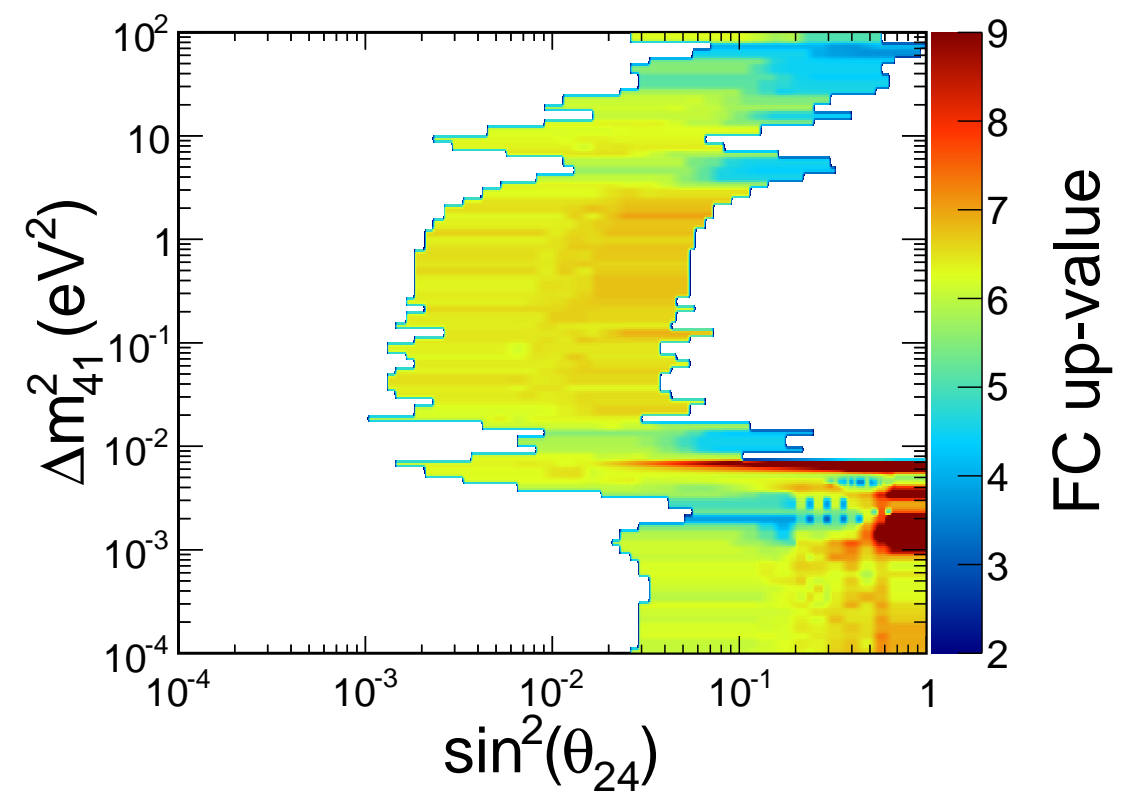

Figure 8.5: Feldman-Cousins correction surface used to construct a 90\% C.L. contour.

Figure 8.6 shows a comparison between the $90 \%$ confidence intervals obtained through using a Global scan and the Feldman-Cousins unified approach for both the data and sensitivity. It can be seen that the Gaussian assumption would result in limits that are too strong. 

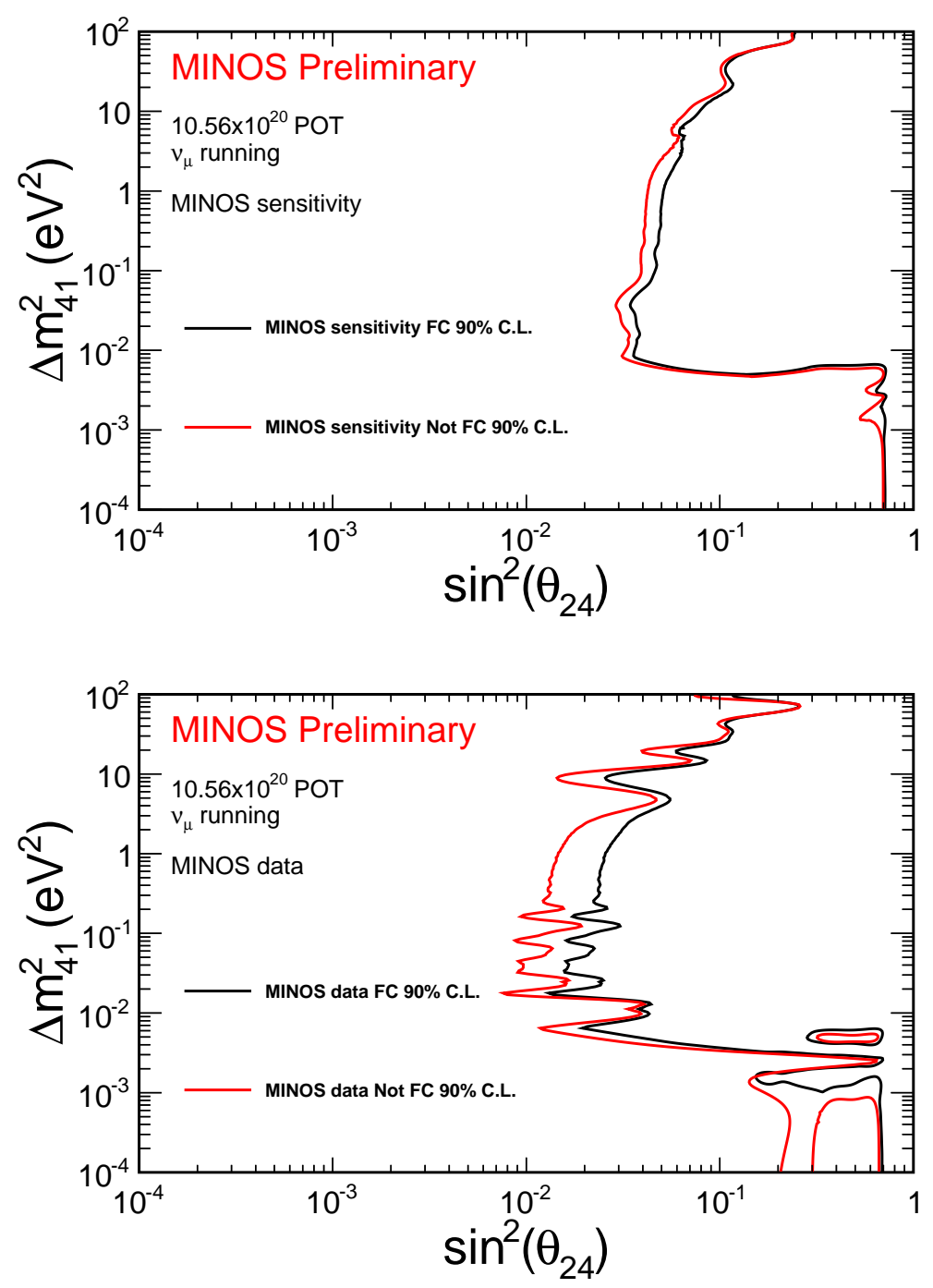

Figure 8.6: Comparison of the 90\% C.L. exclusion contour from simulation (top) and data (bottom) before (red) and after (black) the application of the FeldmanCousins unified approach.

\subsection{Statistical and Systematic Fluctuations in Fake Data}

The MINOS analysis uses a covariance matrix $V$ when fitting the MC and data Far over Near $(\mathrm{F} / \mathrm{N})$ ratios. This matrix contains the bin-to-bin correlations in reconstructed neutrino energy due to the 26 systematics and the statistical uncertainties. 


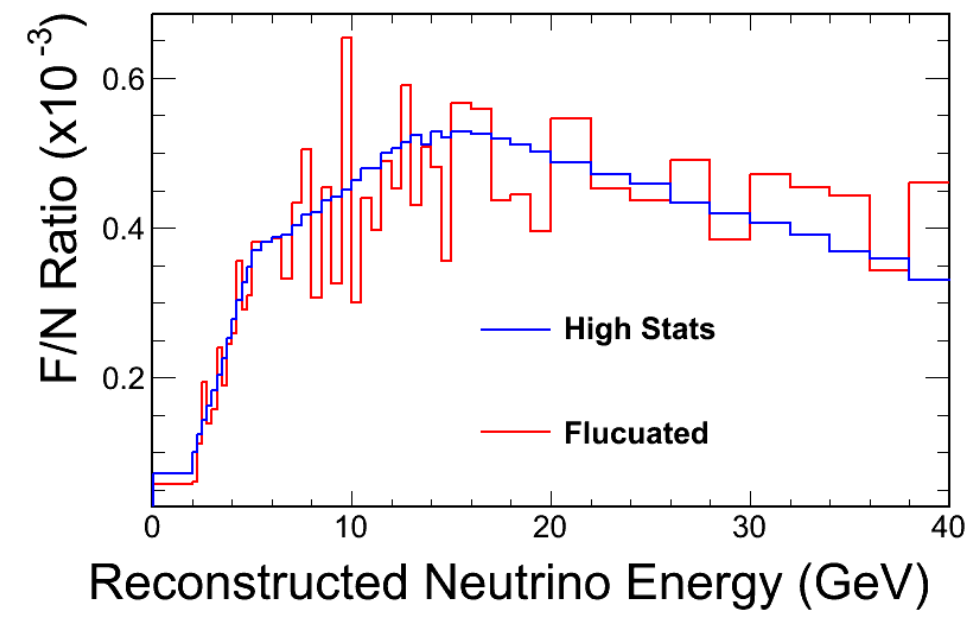

(a) Charged Current F/N Ratio

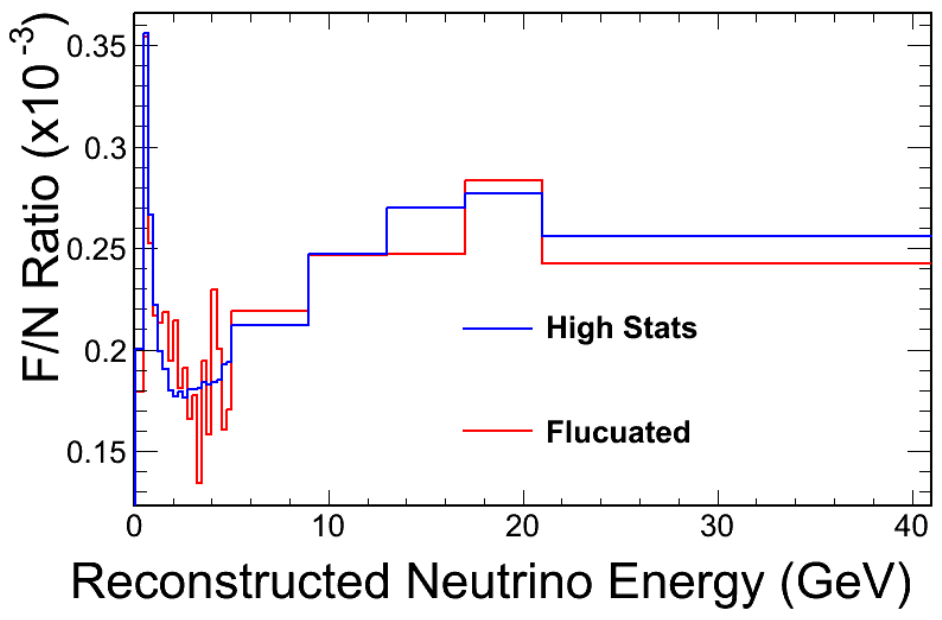

(b) Neutral Current F/N Ratio

Figure 8.7: Figure (a) shows a a statistically and systematically fluctuated CC $\mathrm{F} / \mathrm{N}$ ratio (red) for reconstructed neutrino energy. Figure (b) shows a statistically and systematically fluctuated $\mathrm{NC} \mathrm{F} / \mathrm{N}$ ratio (red) using equation 8.15. The blue lines are high statistics $\mathrm{MC} \mathrm{F} / \mathrm{N}$ ratios produced with standard three flavour parameters along with $\Delta m_{41}^{2}=2.5 \mathrm{eV}^{2}, \theta_{24}=0.2$ radians and $\theta_{34}=\theta_{14}=0$.

The information from this matrix was used to create statistically and systematically fluctuated fake data as shown in figure 8.7. A high-statistics F/N MC ratio is produced $\left(\mathbf{R}^{\mathbf{M C}}\right)$. The covariance matrix is Hermitian and positive-definite, which allows us to decompose it into a product of an upper triangular matrix $\mathbf{L}$ and its transpose as seen in equation 8.14. Think of this as finding the square root of a matrix. 


$$
V=L^{T} L
$$

For every bin, $i$, in $\mathbf{R}^{\mathrm{MC}}$ a number is randomly selected from a normal distribution with $\mu=0$ and $\sigma=1$ known as $\mathbf{y}=\left\{y_{i}\right\}$. The triangular matrix $\mathbf{L}$ is used to transform the random variables $\mathbf{y}$ into a set of correlated numbers. The set of fluctuated variables can be expressed as

$$
\mathbf{R}^{\text {fluc }}=L \mathbf{y}+\mathbf{R}^{\mathrm{MC}} .
$$

Therefore a set of fluctuated fake data can be created by using the correlations between energy bins from the combined systematic and statistical covariance matrix. This process can be performed $N$ times, to produce the fake data used in the construction of the confidence intervals for the Feldman-Cousins unified approach. 


\section{Chapter 9}

\section{Near Detector Data Quality}

\section{Monitoring}

This chapter will discuss the data quality monitoring performed at the Near Detector for the $10.56 \times 10^{20} \mathrm{POT}$ dataset used in this thesis as well as the data quality checks performed during the first two periods of data taking with MINOS+. An intensity effect becomes apparent and any impact on the MINOS sterile neutrino analysis is quantified, in order to justify if action needs to be taken.

\subsection{Data Quality Monitoring at the ND}

It is important to ensure the data being collected is of the highest quality possible in order to reduce any bias and improve energy resolution to ensure a correct understanding of the observed data. The ND, with its proximity to the NuMI target, receives ample statistics for daily data quality checks to ensure the NuMI facility is producing a consistent neutrino beam and that the ND is fully operational. The NuMI beam has its own monitoring devices that monitor the POT delivered per spill, twenty-four capacitative beam position monitors (BPMs) that monitor the batch intensity per spill, and muon stations (ionisation chambers) 


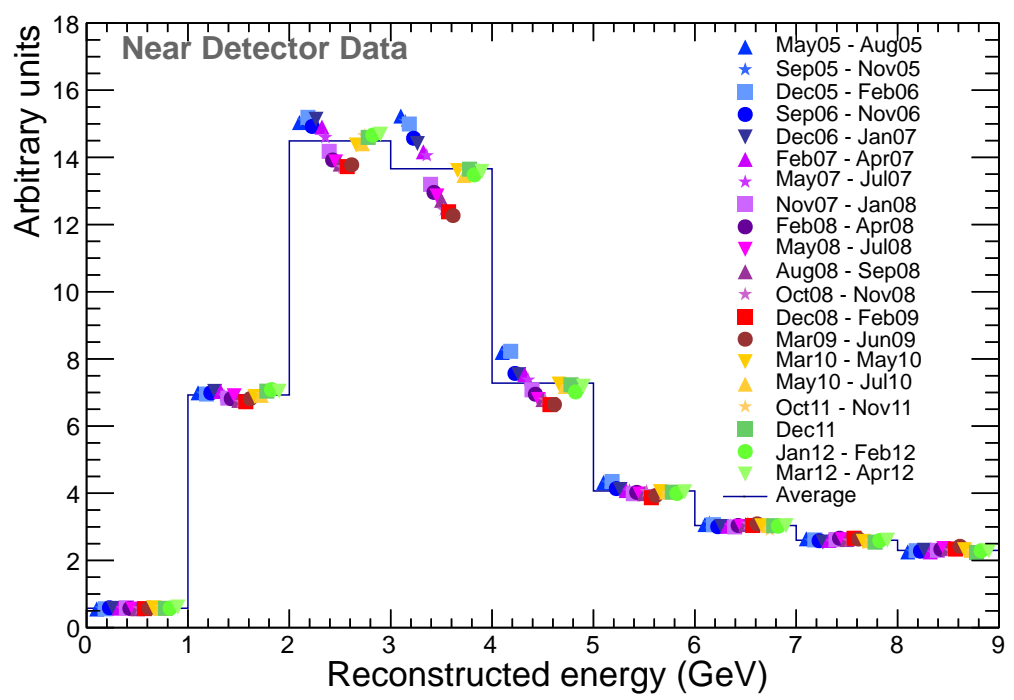

Figure 9.1: The reconstructed neutrino energy spectrum for charged-current events at the ND for runs 1,2, 3, 5, 6 and 10. The histograms are normalised according to their respective POT exposures over time.

that allow measurements of the stability of the neutrino beam.

\subsubsection{Data Quality for the MINOS Era}

Figures 9.1 and 9.2 show the stability of the CC and NC energy spectra for the entire $10.56 \times 10^{20}$ POT dataset used in the sterile neutrino analysis. The data is split up into arbitrary time periods to show how the energy spectrum changes over time.

One can observe a clear distortion in the peak of the energy spectra for both CC and NC events which occurred across Run Periods 2 and 3. This has been discovered to be due to decay of the NuMI target; the high intensity proton beam over time has caused the target to break up and become less dense. This effect can be modelled in Monte Carlo (MC) and is shown to agree with a deficit in the peak of the energy spectrum [85]. The target was replaced for the later runs and the event rate jumps back to a higher value, however not as high as in the original Run 1 due to the inclusion of helium in the decay pipe for health and safety concerns of having a vacuum in such a large space. The effect of helium 


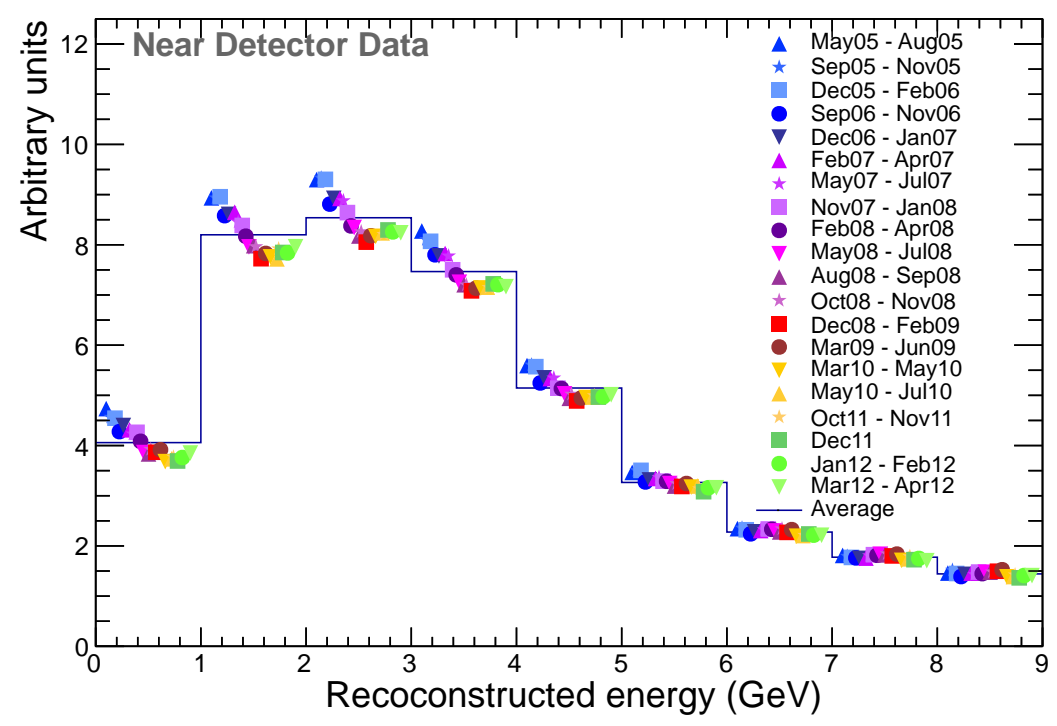

Figure 9.2: The reconstructed neutrino energy spectrum for neutral current events at the ND for runs 1, 2, 3, 5, 6 and 10. The histograms are normalised according to their respective POT exposures over time.

in the decay pipe will reduce the neutrino flux in the peak due to additional collisions of mesons with the helium.

The energy spectra from both the CC and NC samples in figures 9.1 and 9.2 are integrated across two energy windows, $0-6 \mathrm{GeV}$ and $6-200 \mathrm{GeV}$ to produce figures 9.3 and 9.4. This allows the event rate per day to be calculated for each energy window as is shown for both the $\mathrm{CC}$ and $\mathrm{NC}$ samples in figures 9.3 and 9.4. These data quality plots are useful for observing any migration of events from low energies (the peak) to higher energies (past the falling edge of the peak in the CC energy spectrum specifically). A problem with calibration or event reconstruction would shift the energy spectrum during a particular time period and the event rates in the two energy windows would vary; this is a particularly useful tool for observing any day-to-day problems with the quality of the ND data, such as hot channels.

The effect of target decay can also be observed in figures 9.3 and 9.4 showing a gradual reduction in the low-energy event rate over time and a much smaller rise in the high energy window. On a smaller scale, looking at variations in 

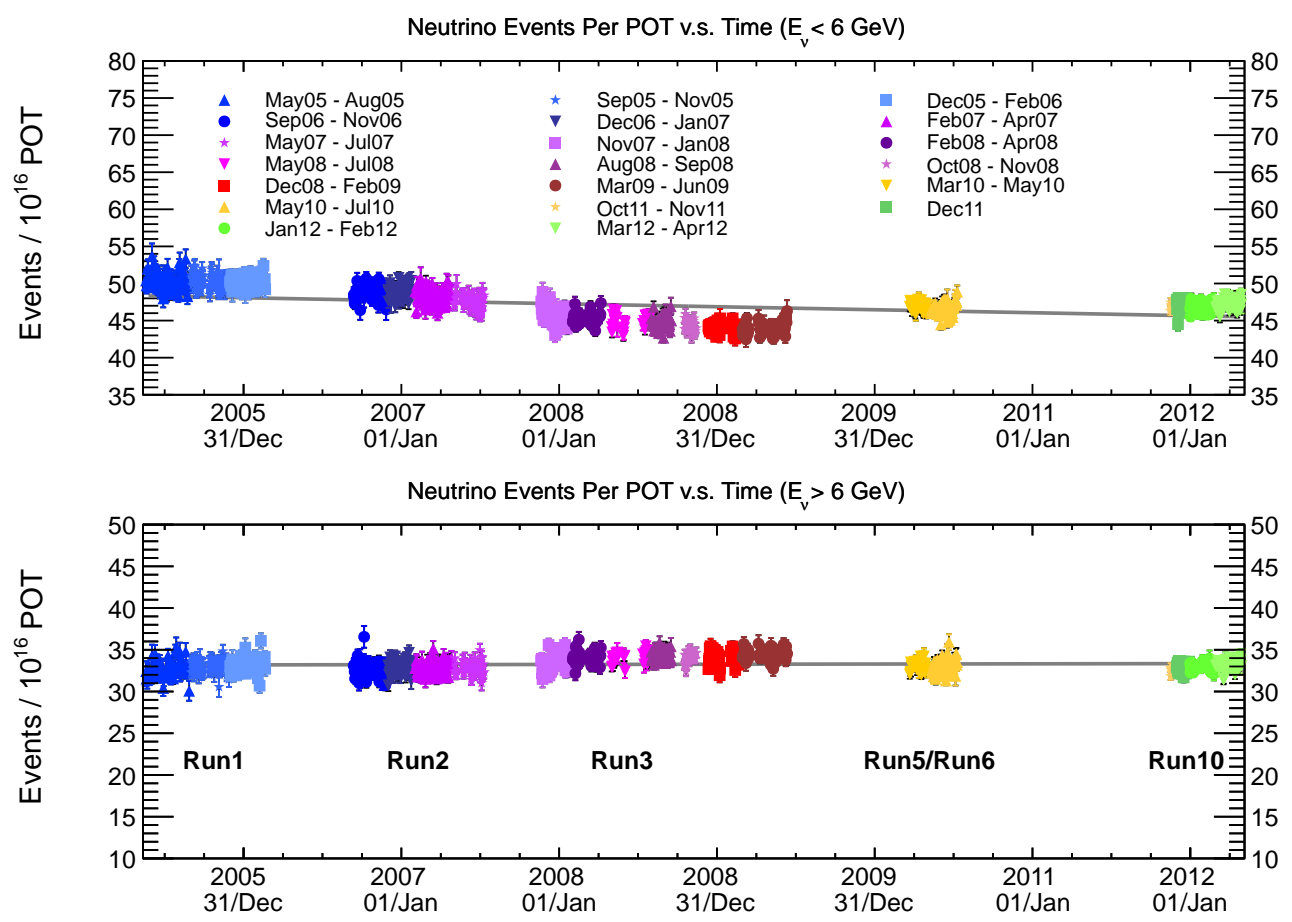

Figure 9.3: Observed rate of CC-like events per POT in the Near Detector binned as a function of time for Run Periods 1, 2, 3, 5, 6 and 10. The top panel shows the event rate for reconstructed energies below $6 \mathrm{GeV}$; the bottom panel shows the event rate for energies above $6 \mathrm{GeV}$. The grey is a straight-line fit to the data.

event rate across the individual runs it is observed that the data is stable. It is worth pointing out to the reader than any effects of target decay do not affect the analysis presented in this thesis due to the use of the $\mathrm{F} / \mathrm{N}$ ratio in the fit: the effect of target decay would affect both detectors identically, and is cancelled out in the $\mathrm{F} / \mathrm{N}$ ratio.

\subsubsection{Data Quality for the MINOS+ Era}

MINOS+ began taking data in September 2012 after the upgrade to the NuMI facility to produce a neutrino beam in the medium-energy configuration. The data quality monitoring plots must be altered by shifting the energy windows for the event rate vs time graphs to $0-8 \mathrm{GeV}$ and $8-200 \mathrm{GeV}$ due to the shift in the beam peak. This thesis will show the data stability for the MINOS+ experiment for the first two years of running denoted as Runs 11 and 12. Figure 9.5 shows 

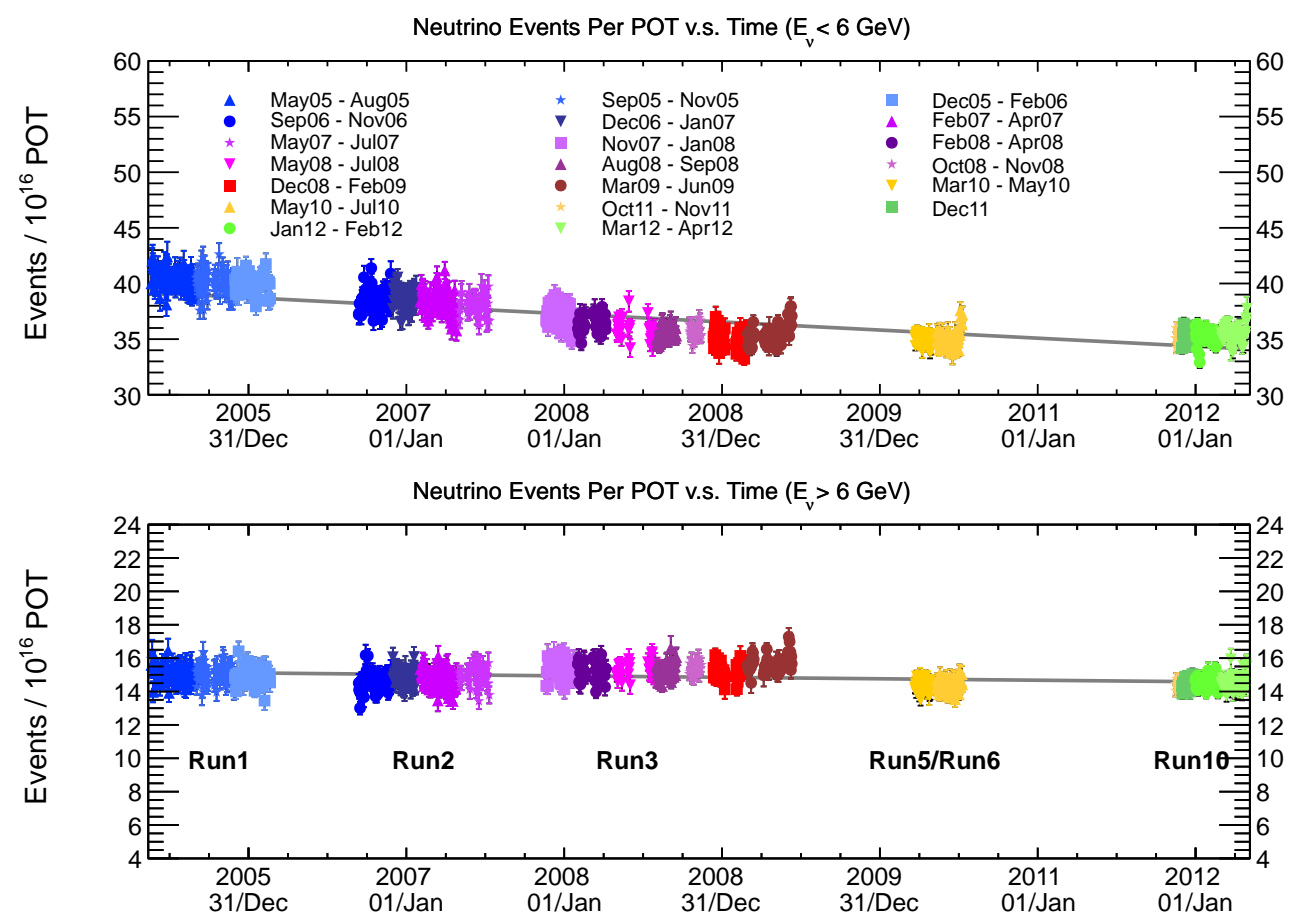

Figure 9.4: Observed rate of NC-like events per POT in the Near Detector binned as a function of time for Run Periods 1, 2, 3, 5, 6 and 10. The top panel shows the event rate for reconstructed energies below $6 \mathrm{GeV}$; the bottom panel shows the event rate for energies above $6 \mathrm{GeV}$. The grey is a straight-line fit to the data.

the CC energy stability plot for the MINOS+ ND during Run Period 11. The corresponding event rate vs time metric is displayed in figure 9.6. The data quality for MINOS+ Run Period 11 shows a stable period of data with no signs of target decay or problems with calibration or reconstruction.

Figures 9.7 and 9.8 show the data quality monitoring plots for Run Period 12. During Run Period 12 in the month of March 2015 slip stacking was in operation at the NuMI facility (see chapter 3). This correlated with a decrease in the event rate per POT, particularly in the peak of the CC energy spectrum (similar effects for the NC spectrum were seen but are not shown here). Figure 9.8 is overlaid with the average intensity of the NuMI beam for each day; a correlation can be seen between intensity and event rate per POT. This is indicative of pile-up at the ND due to a saturation in events resulting in the reconstruction software failing to reconstruct all potential events. Some low-energy events are possibly 


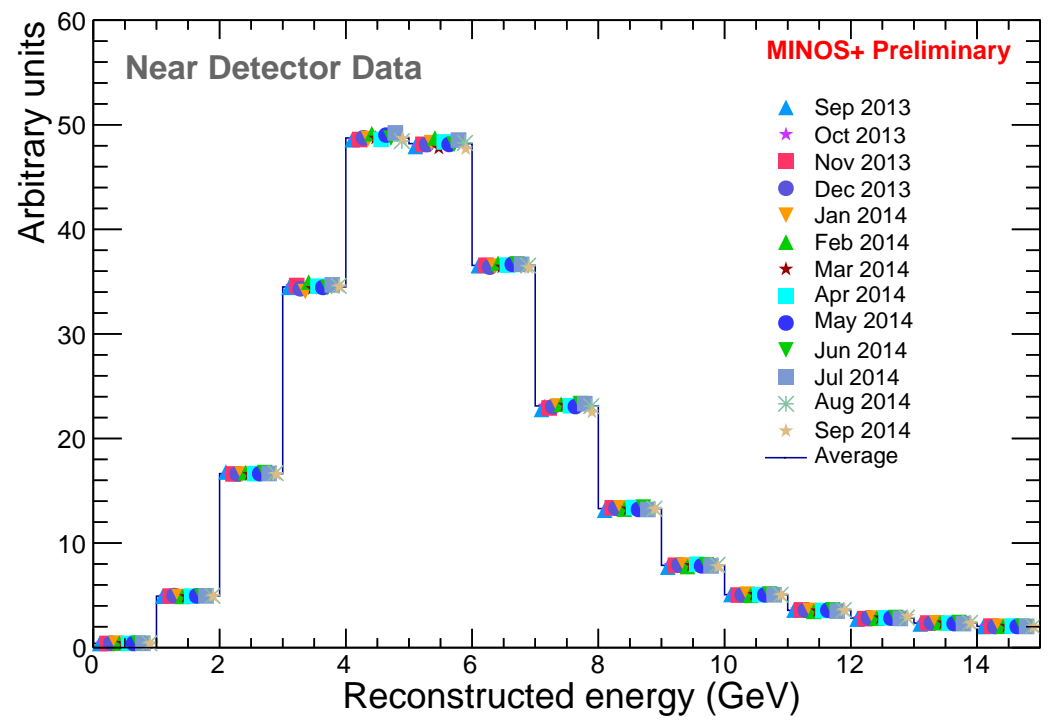

Figure 9.5: The reconstructed neutrino energy spectrum for charged current events at the ND for Run 11 (MINOS+). The histograms are normalised according to their respective POT exposure over time.
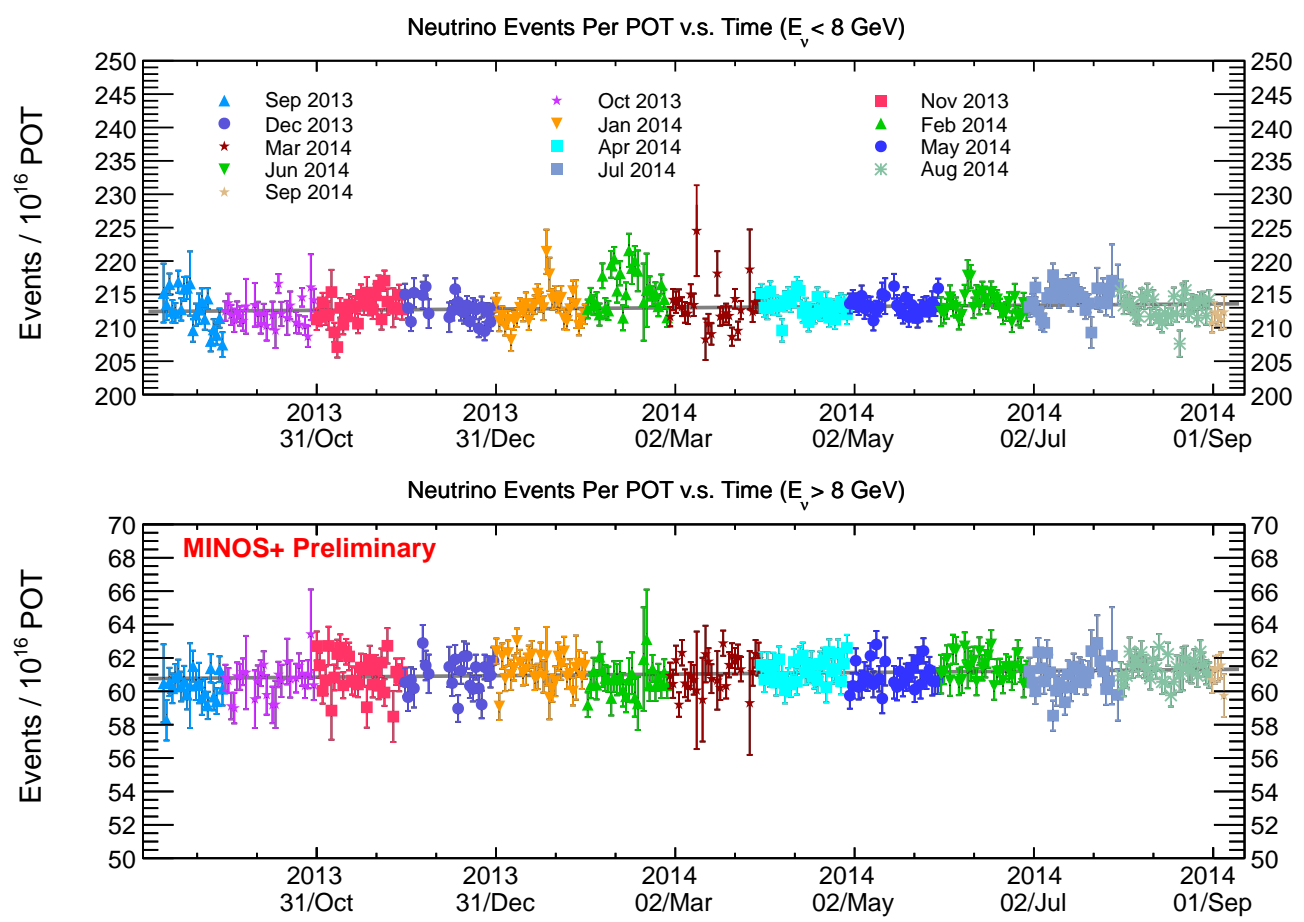

Figure 9.6: Observed rate of CC-like events per POT in the Near Detector binned as a function of time for Run 11. The top panel shows the event rate for reconstructed energies below $8 \mathrm{GeV}$; the bottom panel shows the event rate for energies above $8 \mathrm{GeV}$. 


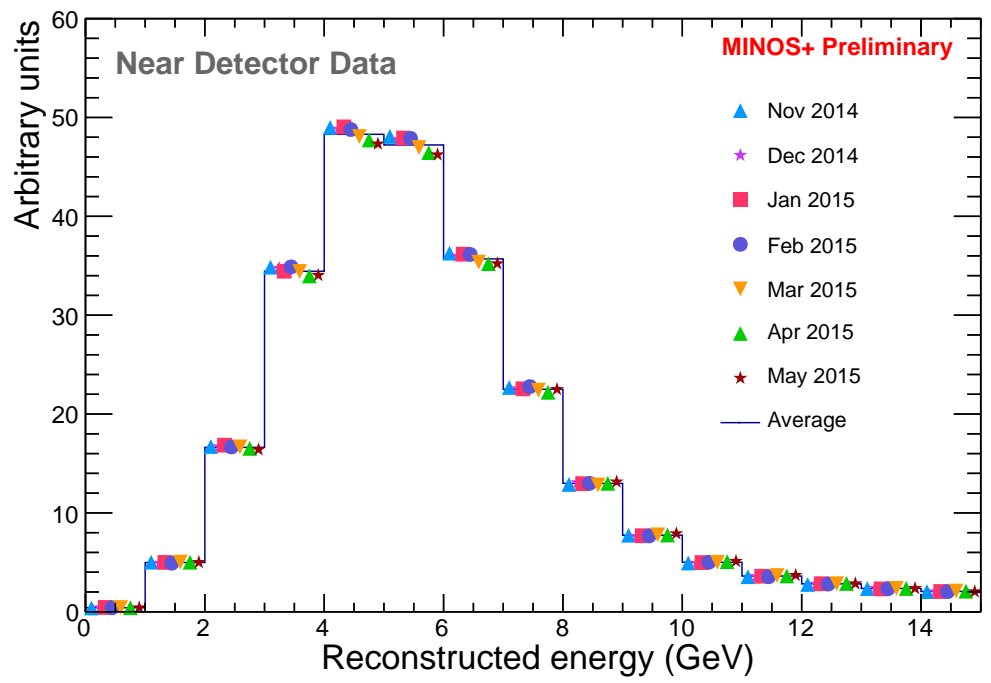

Figure 9.7: The reconstructed neutrino energy spectrum for charged current events at the ND for Run 12 (MINOS+). The histograms are normalised according to their respective POT exposure over time.

being merged with high-energy events, giving rise to the slight increase in the high-energy window.

Unlike target decay the effect of pile up due to a higher intensity NuMI beam affects only the ND which will bring about a bias in the $\mathrm{F} / \mathrm{N}$ ratio for future sterile neutrino analyses at MINOS+. This effort of addressing pile-up at the ND for MINOS+ is ongoing. A study to assess any intensity effects for the MINOS-era data is presented in the next section. 

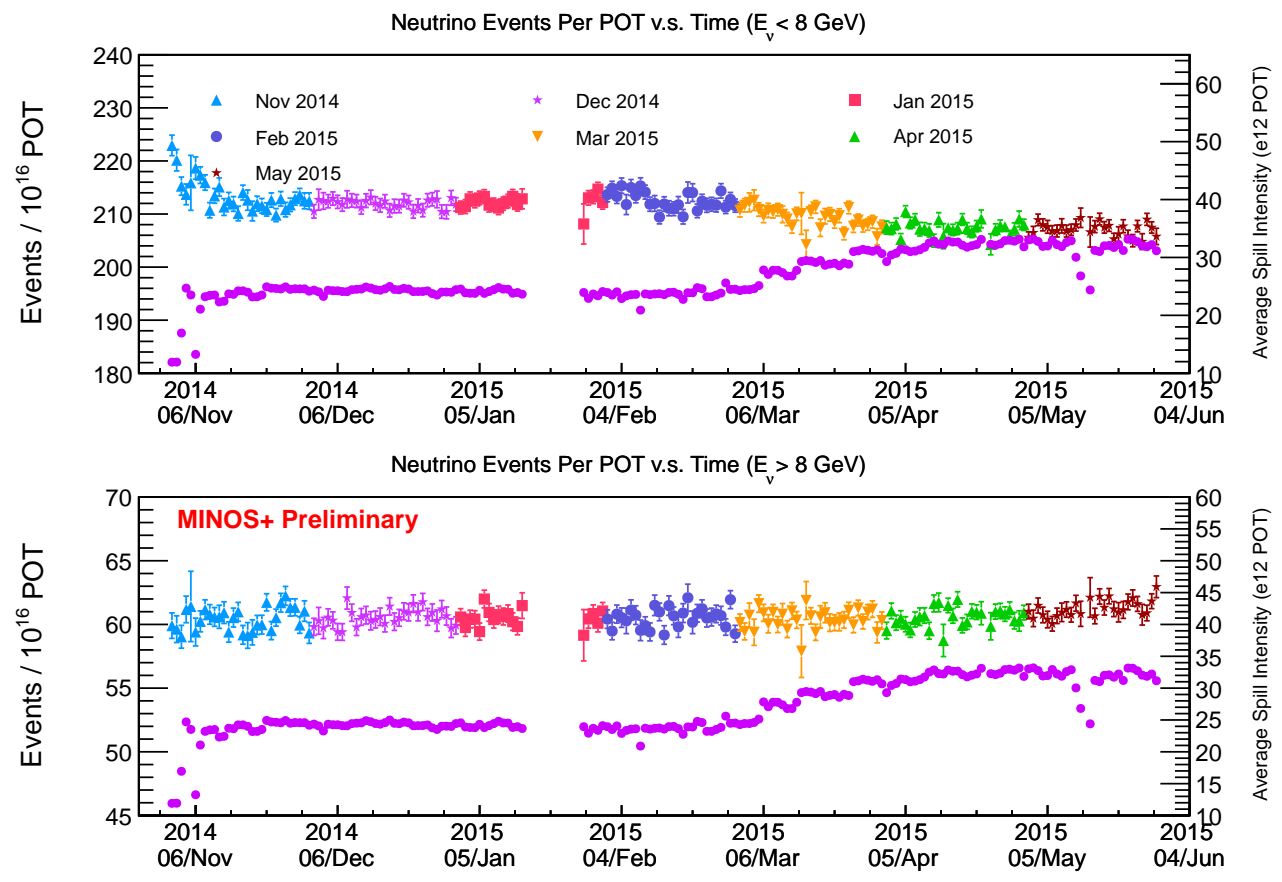

Figure 9.8: Observed rate of CC-like events per POT in the Near Detector binned as a function of time for Run 12. The top panel shows the event rate for reconstructed energies below $8 \mathrm{GeV}$; the bottom panel shows the event rate for energies above $8 \mathrm{GeV}$. The purple points overlaid are the average intensity (defined as the number of protons per pulse) of the NuMI beam for each day. 


\subsection{Does MINOS need an Intensity Correction?}

There have been previous attempts to quantify how sensitive the reconstruction software is to the intensity at the ND [138]; these studies have shown that no significant effect can be observed that correlates the intensity to the number of reconstructed events in a spill that is not already modelled by MC.

Recent data quality plots for MINOS+ have highlighted that there is an intensity effect and it is believed to originate from the batch structure within a beam spill. The MC at MINOS did not take into account the batch structure of an individual spill shown earlier in figure 3.9; instead an average is taken to be constant throughout the spill. That is not to say that a Run will not have different average spill intensities; this will be discussed later.

The shapes of the ND energy spectra depend on the instantaneous proton intensity. Although this effect is well-modelled it could potentially pose a problem for the sterile analysis since the MC was not matched on a batch-by-batch level (a potential problem due to slip-stacked batches). For example a spill with an intensity of $36 \times 10^{12} \mathrm{ppp}$ (protons-per-pulse) with six batches of equal intensity would give each batch an intensity of $6 \times 10^{12} \mathrm{ppb}$ (protons-per-batch). However slip-stacking may result in some batches having double the intensity of others as shown in figures $9.9-9.14$. To deal with this, a set of corrective weights need to be calculated that would be applied to the MC such that it corresponds to the instantaneous intensity of the data more accurately.

Figures $9.9-9.14$ show the batch structure for the $10.56 \times 10^{20}$ POT dataset used in the sterile neutrino analysis. The data is taken in either a five-batch mode (the sixth batch would of been delivered to the Tevatron) or a six-batch mode. 

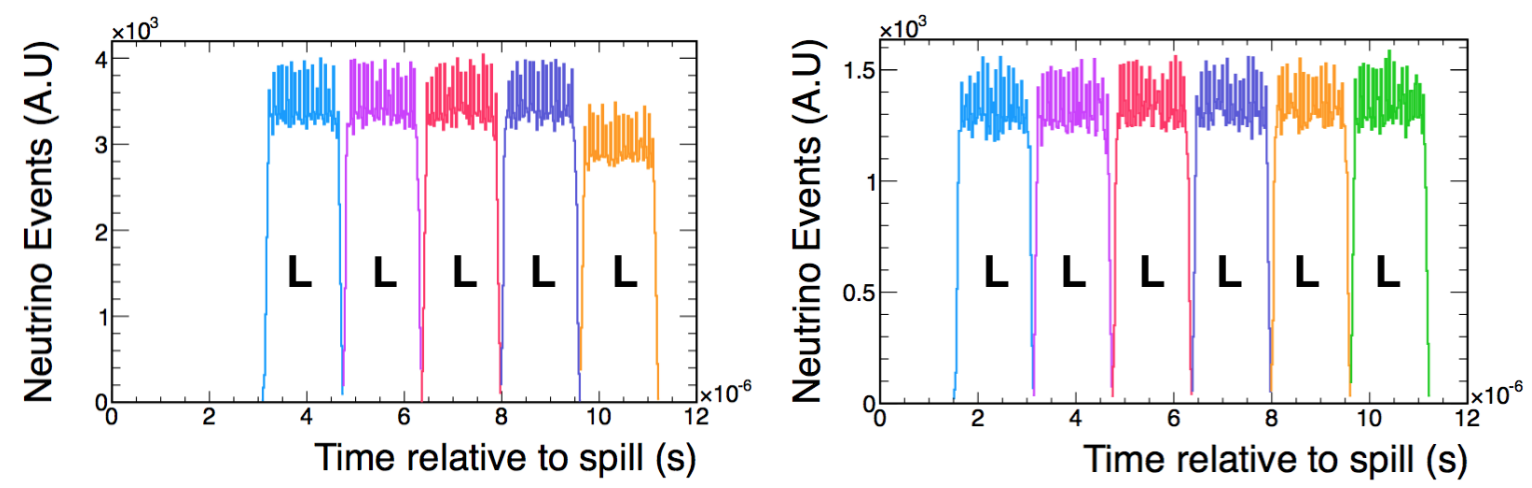

Figure 9.9: MINOS Run Period 1 batch structure for five-batch mode (left) and six-batch mode (right). Each batch is labelled as either a high-intensity batch $(\mathrm{H})$ or a low-intensity batch $(\mathrm{L})$.
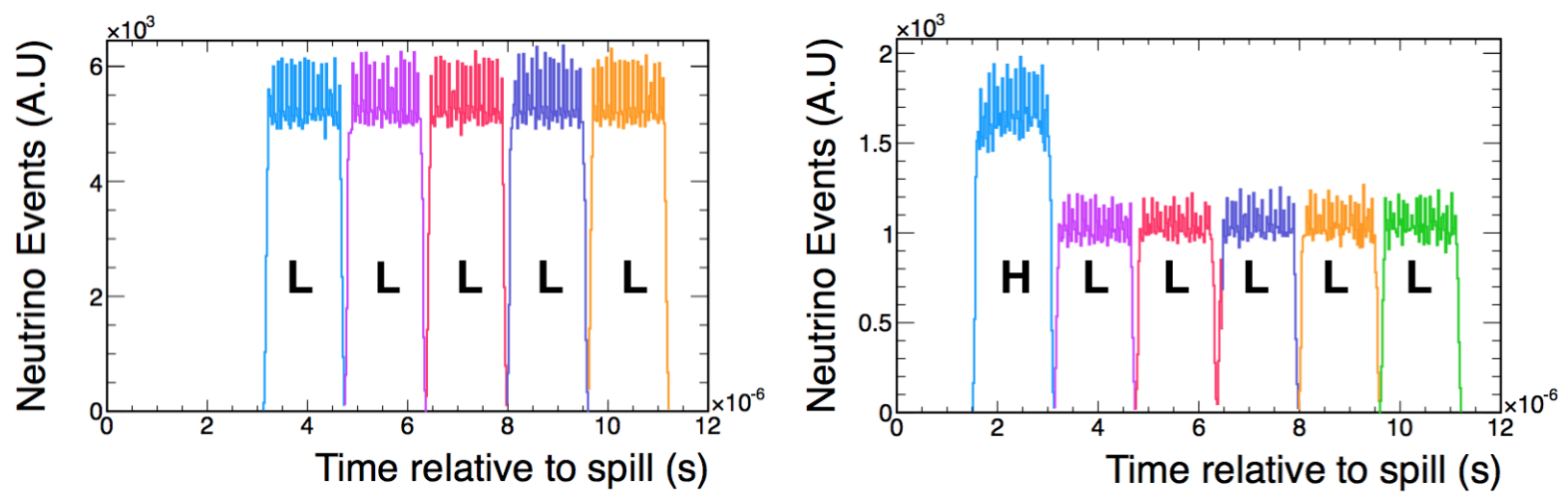

Figure 9.10: MINOS Run Period 2 batch structure for five-batch mode (left) and six-batch mode (right). Each batch is labelled as either a high-intensity batch $(\mathrm{H})$ or a low-intensity batch (L).
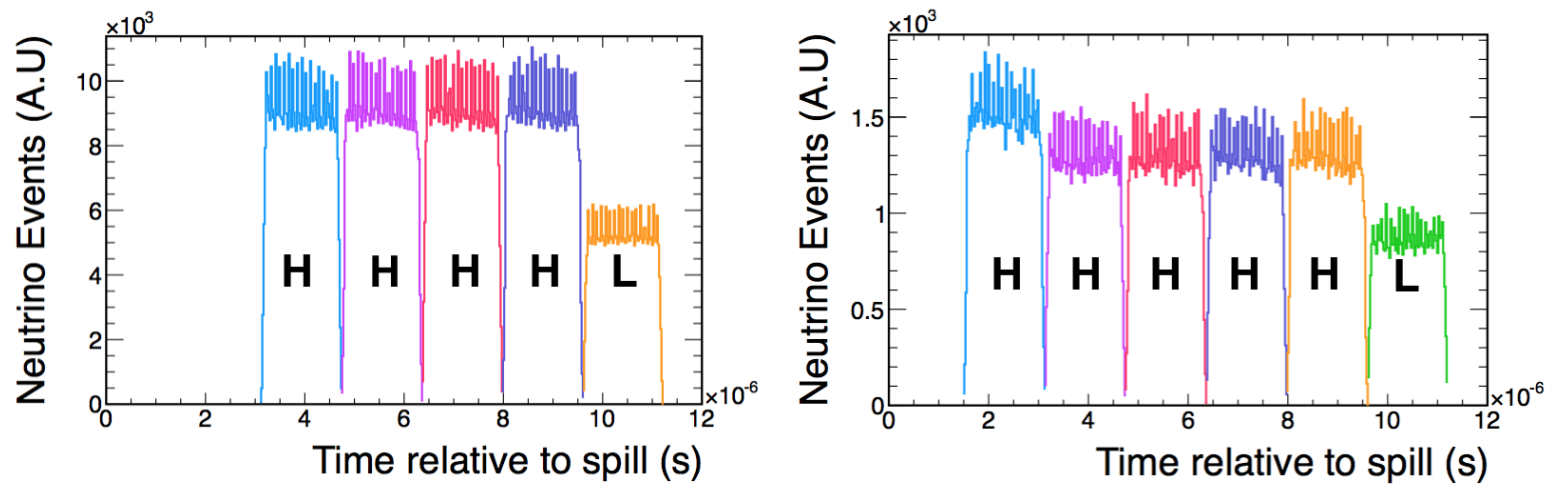

Figure 9.11: MINOS Run Period 3 batch structure for five-batch mode (left) and six-batch mode (right). Each batch is labelled as either a high-intensity batch (H) or a low-intensity batch (L). 

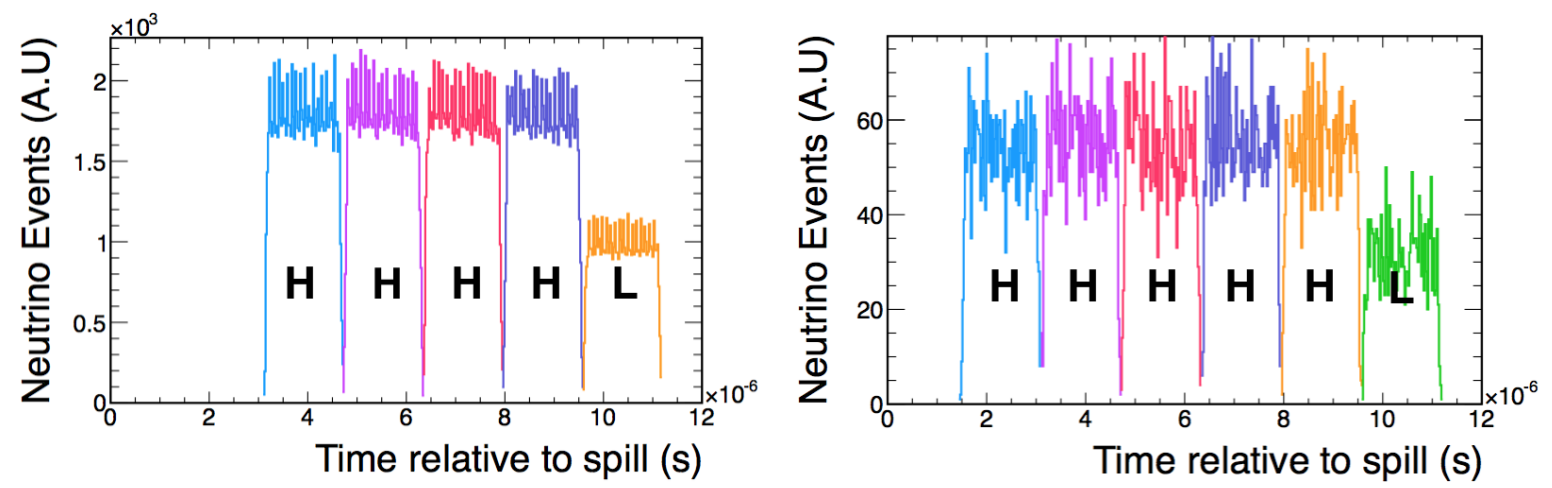

Figure 9.12: MINOS Run Period 5 batch structure for five-batch mode (left) and six-batch mode (right). Each batch is labelled as either a high-intensity batch $(\mathrm{H})$ or a low-intensity batch (L).
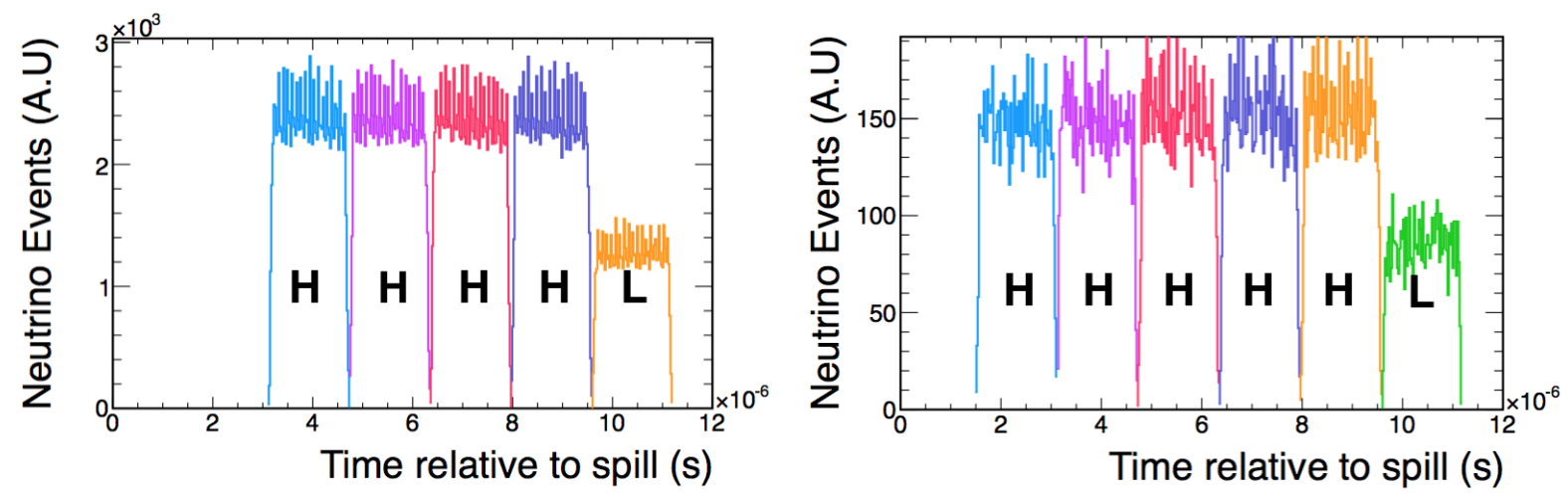

Figure 9.13: MINOS Run Period 6 batch structure for five-batch mode (left) and six-batch mode (right). Each batch is labelled as either a high-intensity batch (H) or a low-intensity batch (L).
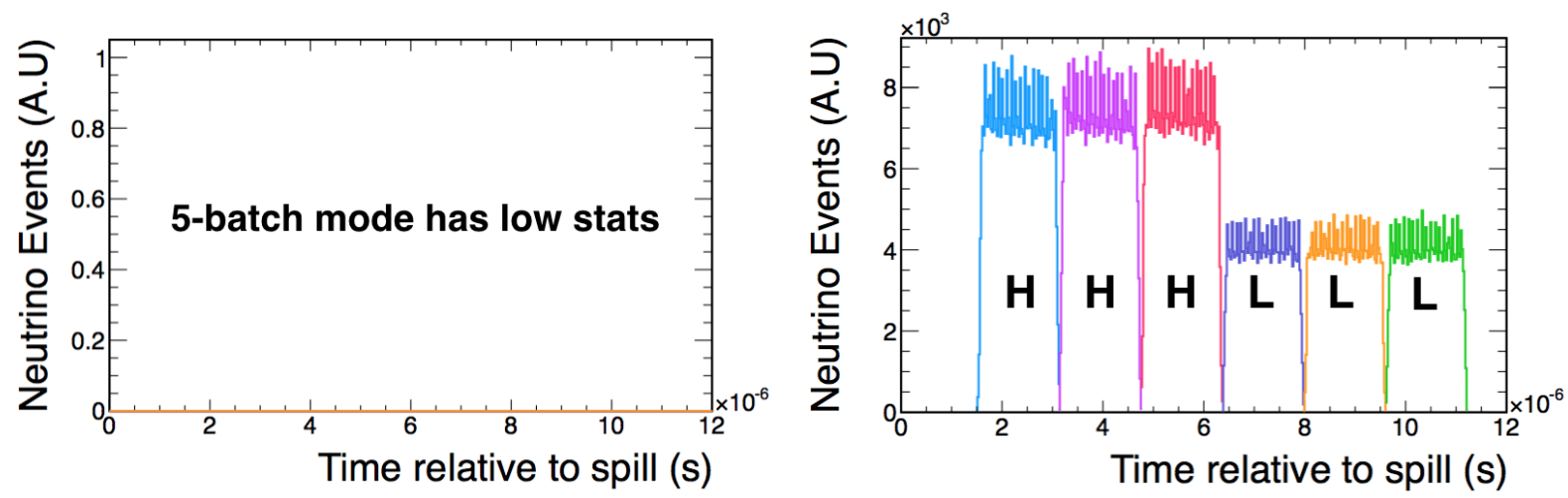

Figure 9.14: MINOS Run Period 10 batch structure for five-batch mode (left) and six-batch mode (right). Each batch is labelled as either a high-intensity batch $(\mathrm{H})$ or a low-intensity batch $(\mathrm{L})$.

A summary of what proportion of the data is in each Run for a high-intensity batch (slip stacked) or a low-intensity batch (nominal) is shown in figure 9.15. It 


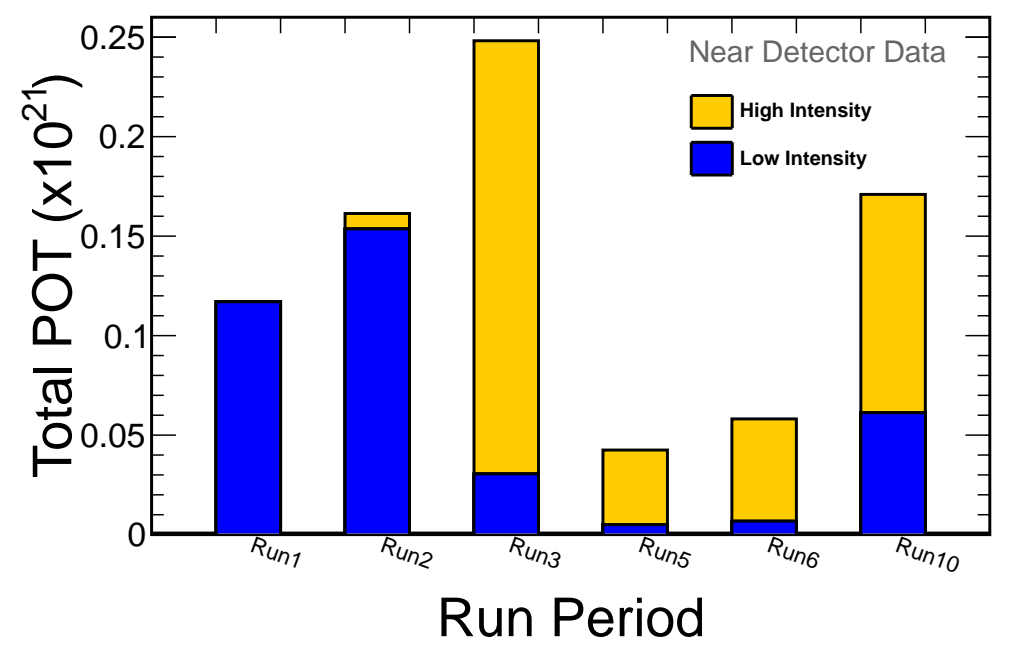

Figure 9.15: A summary of the composition of the MINOS data for all lowenergy neutrino running showing the division of each Run period into high- and low-intensity batches.

is clear that much of the data is a combination of both high- and low-intensity batches particularly in Run 10. This motivates a correction to the MC such that the intensity models the data more accurately.

\subsection{Calculating the Intensity Corrections}

In order to obtain the correct spectral shape for any intensity correction to the ND MC, several MC samples at different intensities were produced. The intensities chosen were: $24,30,48$, and $60 \times 10^{12} \mathrm{ppp}$. The simulation was processed and reconstructed identically to data. Figure 9.16 shows the ND reconstructed neutrino energy spectra produced from MC for both CC and NC selected events. The MC produced used the Run Period 3 conditions, however the choice was arbitrary as the corrections will be derived and applied to the $\mathrm{F} / \mathrm{N}$ ratios; any differences between each Run Period are effectively cancelled out. Figure 9.16 shows that the MC simulates the intensity effect as the number of events situated within the energy peak decreases as the ND experiences pile-up with increased intensity. 

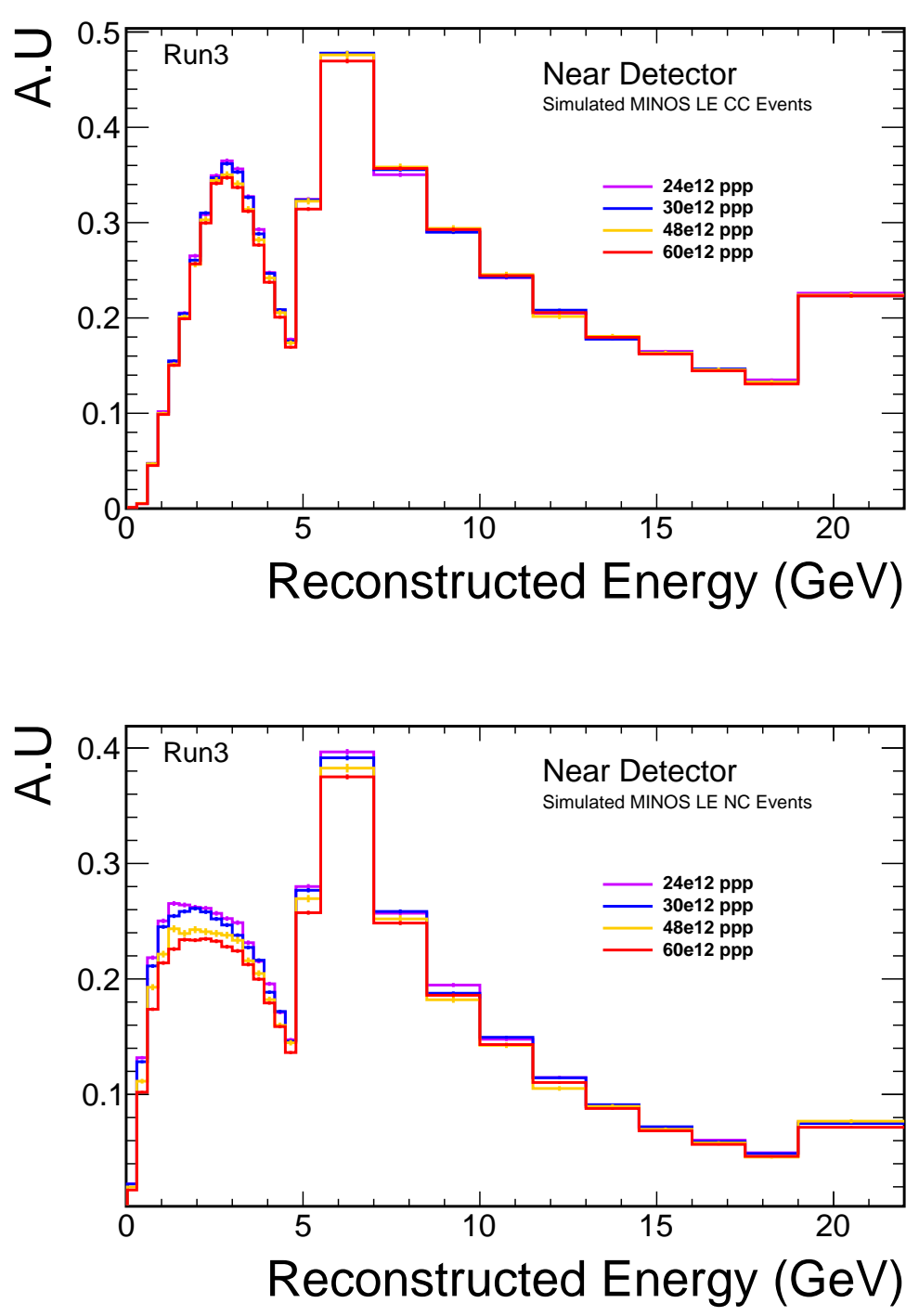

Figure 9.16: Simulated energy spectra of charged- (top) and neutral-current (bottom) events (Run 3) for four different intensities: 24, 30, 48, and $60 \times 10^{12} \mathrm{ppp}$. 

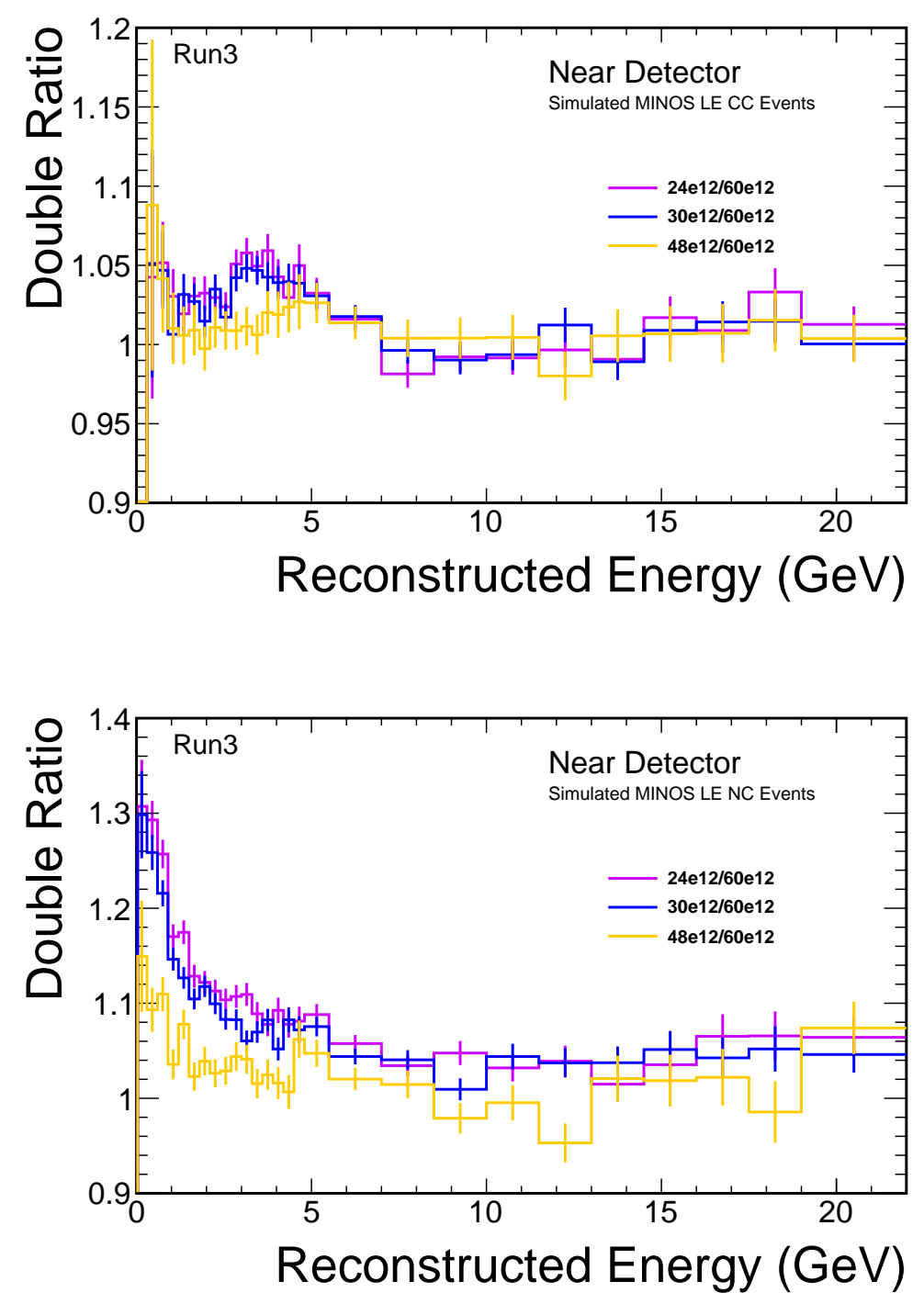

Figure 9.17: Ratios of simulated Near Detector energy spectra between high and low intensities, for both charged- (top) and neutral-current (bottom) events.

The essence of this method is that the ratio of a high-intensity MC sample to a low-intensity MC sample, $R$, tells us how the ND energy spectrum varies as a function of intensity for both CC and NC samples. Figure 9.17 shows the ratio of the three lower intensities to the highest intensity for both the $\mathrm{CC}$ and $\mathrm{NC}$ samples. This ratio gives the energy-dependent shape of the intensity effect and will form the basis of the corrections to MC.

In order to correctly scale the corrections in figure 9.17 for each Run Period $j$, the difference is taken between the mean intensity of the nominal MC for that 
Run Period, $I_{\mathrm{MC}, j}$, shown in figure 9.18, and the mean intensity of the data, $I_{\text {Data }, j}$, obtaining the mean intensity shift for that Run, $S_{j}=I_{\text {Data }, j}-I_{\mathrm{MC}, j}$. The difference between a high- and low-intensity pair in figure 9.17 has the fixed value $D$. The correction factor, $R_{\text {corr }, j}$, is computed by scaling the spectral ratio $R$ for this high- and low-intensity pair from figure 9.17 by $S_{j} / D$. This scaled ratio will then be applied to the energy spectrum of the nominal $\mathrm{MC}$ that goes into the analysis for each Run Period $j$.

The mean intensity of each MC Run Period is calculated from the histograms given in figure 9.18; the histograms show for each Run Period the proportion of spills at a given intensity.

Table 9.1 summarises the mean per-batch intensity in the data for each Run Period, $I_{\text {Data }, j}$. The per-batch intensity is given in units of protons per batch (ppb). To construct this table, a histogram of the ppb in every batch of the Run Period $j$ is made, as shown in Figure 9.19. These histograms each show either two or three peaks. The mean intensities of each of these peaks, along with the POT fraction of the data in each peak, is tabulated in table 9.2.

The mean per-batch intensity of the data, $I_{\text {Data }, j}$, in each Run Period must now be compared with the intensity of the $\mathrm{MC}$ used for that Run Period, $I_{\mathrm{MC}, j}$. The number used to quantify the intensity of the $\mathrm{MC}$ must use the same definition as the data: it must be the instantaneous intensity, i.e. the number of protons arriving in a given time interval. Each proton batch in the data lasts for 81 buckets of $18.83 \mathrm{~ns}$ in length, giving a total batch length of $1525.23 \mathrm{~ns}$. In the MC, the protons in the spill are spread evenly over a time window of 8098 ns. Therefore, to convert the $\mathrm{MC}$ intensity from ppp into ppb units that can be compared to the data intensity numbers in Table 9.1, the MC ppp number must be multiplied by $1525.23 / 8098=1 / 5.3093$.

The second and third columns of Table 9.2 compare the mean data intensity to the mean $\mathrm{MC}$ intensity in each Run Period. These mean ppp numbers are 


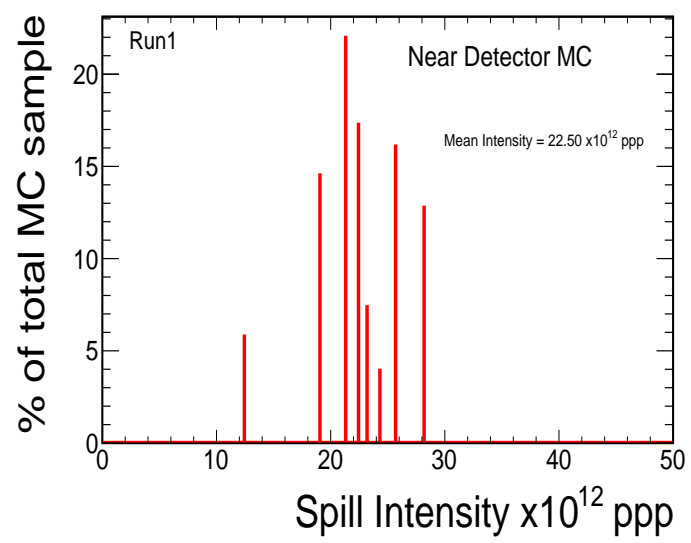

(a) Run 1

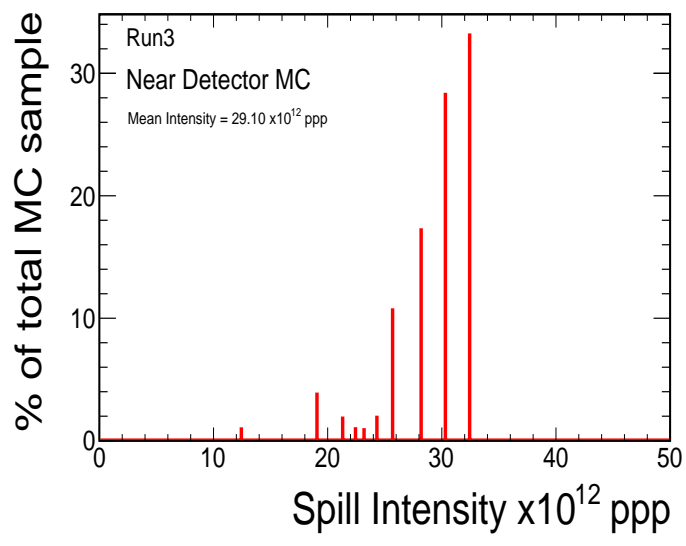

(c) Run 3

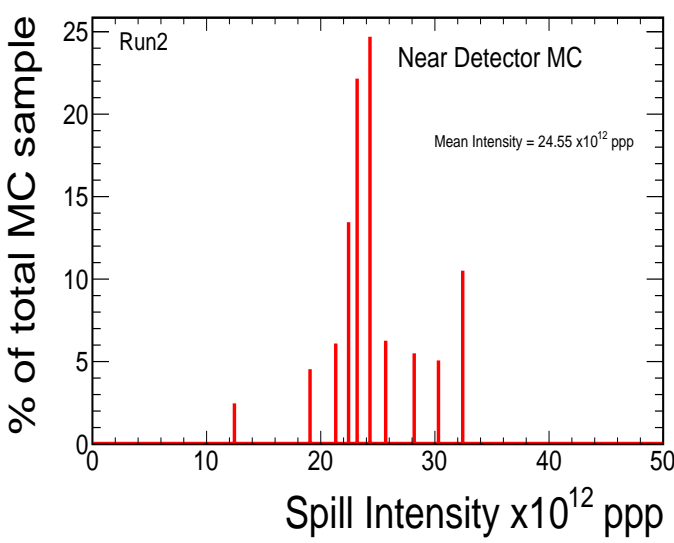

(b) Run 2

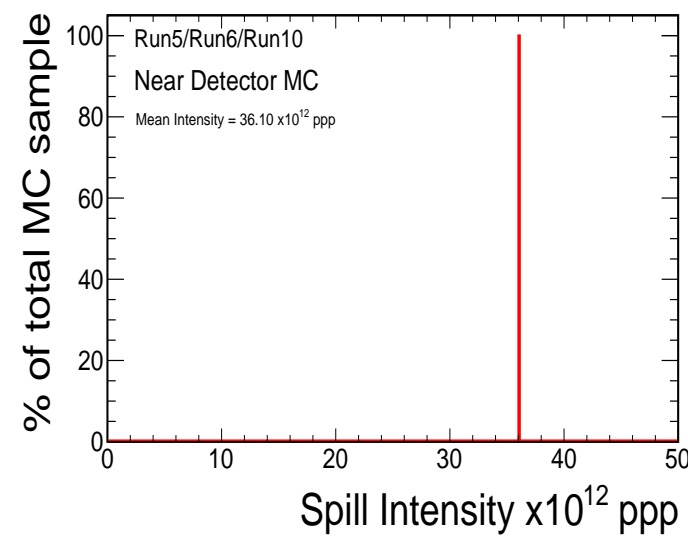

(d) Runs 5, 6 and 10

Figure 9.18: Proportions of intensities used in the Near Detector Monte Carlo for all low-energy neutrino running. 


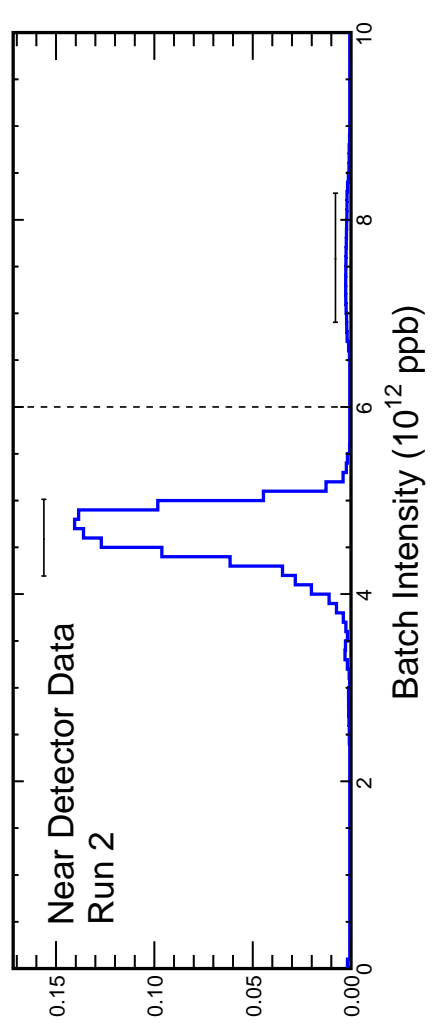

(เ2 $\mathrm{Ol}$ ) $\perp \mathrm{Od}$

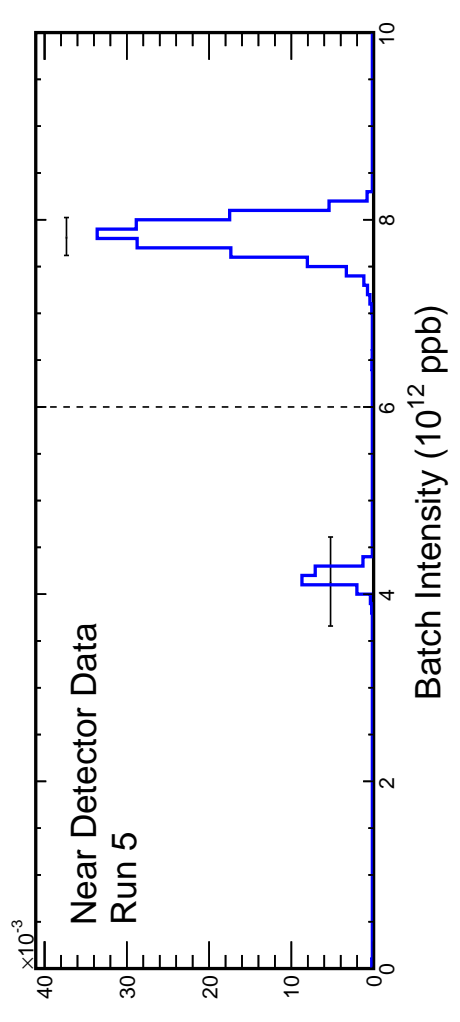

(เzOt) $\perp \mathrm{Od}$

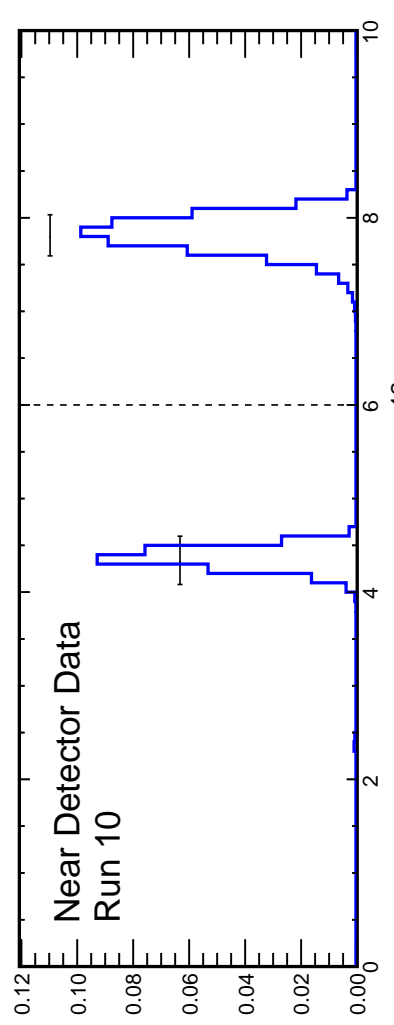

(란) $\perp$ Od

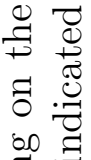
$\stackrel{\circ}{\exists}$.

을 융

$\therefore$ 웜

त

क

离 虽

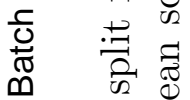

. 5 ह

焉

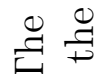

을

当

인

过

Ð
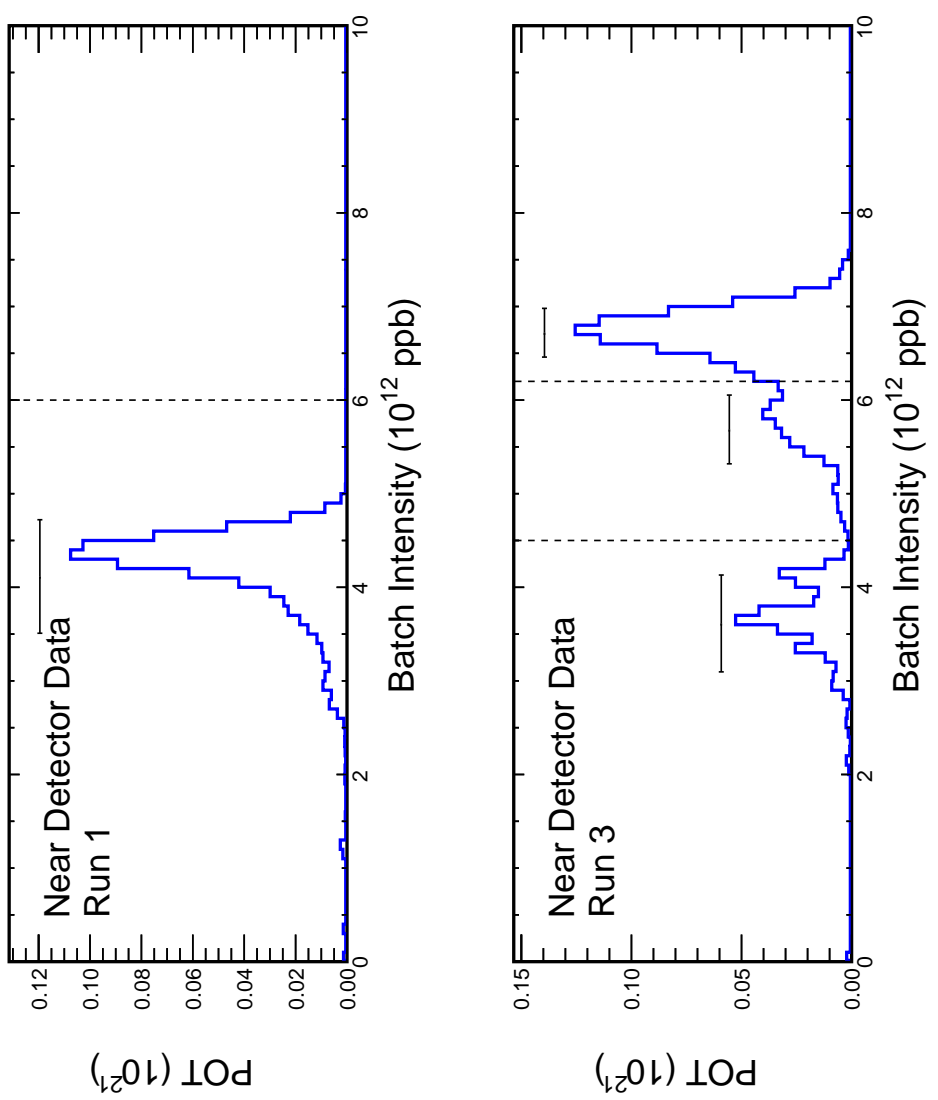


\begin{tabular}{|c|c|c|c|}
\hline $\begin{array}{c}\text { Run period, } \\
j\end{array}$ & $\begin{array}{l}\text { Per-batch } \\
\text { intensity } \\
/ 10^{12} \mathrm{ppb}\end{array}$ & PoT fraction & $\begin{array}{c}\text { Mean per-batch } \\
\text { intensity for run period, } \\
I_{\text {Data }, j} / 10^{12} \mathrm{ppb}\end{array}$ \\
\hline Run I & $\begin{array}{l}4.1154 \\
6.7571\end{array}$ & $\begin{array}{l}0.9998 \\
0.0002\end{array}$ & 4.1159 \\
\hline Run 2 & $\begin{array}{l}4.6036 \\
7.5945\end{array}$ & $\begin{array}{l}0.9518 \\
0.0482\end{array}$ & 4.7478 \\
\hline Run 3 & $\begin{array}{l}3.6182 \\
5.6924 \\
6.7198\end{array}$ & $\begin{array}{c}0.234 \\
0.2173 \\
0.5487\end{array}$ & 5.7708 \\
\hline Run 5 & $\begin{array}{c}4.135 \\
7.8214\end{array}$ & $\begin{array}{l}0.1229 \\
0.8771\end{array}$ & 7.3683 \\
\hline Run 6 & $\begin{array}{l}3.9111 \\
4.8755 \\
7.6565\end{array}$ & $\begin{array}{l}0.1244 \\
0.0678 \\
0.8078\end{array}$ & 7.0020 \\
\hline Run 10 & $\begin{array}{l}4.3398 \\
7.8125\end{array}$ & $\begin{array}{c}0.3653 \\
06347\end{array}$ & 6.5439 \\
\hline
\end{tabular}

Table 9.1: In the data, each Run Period consists of proton batches that divide into either two or three groups of similar intensity, as shown in figure 9.19. The central two columns show the mean ppb of these groupings and the POT fraction, within the Run Period, of data in that grouping. The right-hand column takes a weighted average of the per-batch intensities to give the mean per-batch intensity of the data in that Run Period, $I_{\text {Data }, j}$. 


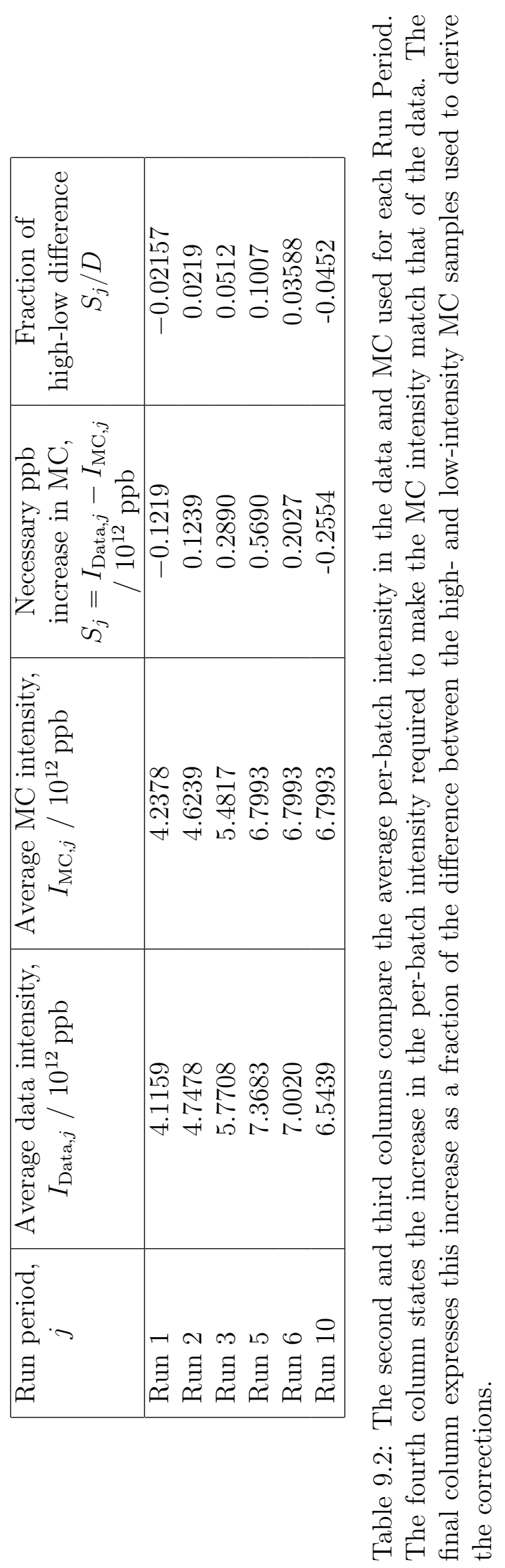


scaled by $1 / 5.3093$ to obtain the mean MC ppb numbers given in Table 9.2. The fourth column then shows by how much the mean MC ppb must be increased to match the data ppb. This number is the variable $S_{j} / D$ referred to earlier.

To obtain the variable $D$, high-intensity and low-intensity MC samples are required. The two samples used have per-spill intensities of $60 \times 10^{12} \mathrm{ppp}$ and $30 \times 10^{12} \mathrm{ppp}$. These correspond to $11.30 \times 10^{12} \mathrm{ppb}$ and $5.64 \times 10^{12} \mathrm{ppb}$ respectively (obtained by scaling the per-spill numbers by $1 / 5.3093$ ). The number $D$ is then the difference between these two per-batch intensities, or $5.64 \times 10^{12} \mathrm{ppb}$. Therefore for each Run Period $j$, the spectral ratios for CC and NC samples corresponding to their two intensities shown in figure 9.17 (the blue lines) were scaled by $S_{j} / D$ to form the actual corrections. These corrections are shown in figure 9.20 .

The corrections in figure 9.20 would be applied to the MC for each Run Period, however the magnitude of these corrections is small. In comparison to the systematic error band (see chapter 6) the corrections can be considered negligible. This is not a surprising result as the original method used to generate the MC by taking the average spill intensity for each run for MINOS is a good approximation to the intensity profile of the data. It was therefore decided to not apply these corrective factors to the MINOS analysis as the final result would not be significantly affected due to the relativity large size of the systematics assessed for the MC. However, the technique presented here will be vital in the MINOS+ era where the pile-up effects are much bigger.

\subsection{Proof of Linearity}

This method of calculating the correction factors relies on the assumption that the intensity change is approximately linear over the range of intensities in which we are working $\left(4.12 \times 10^{12} \mathrm{ppb}-11.30 \times 10^{12} \mathrm{ppb}\right)$. It is this assumption of 

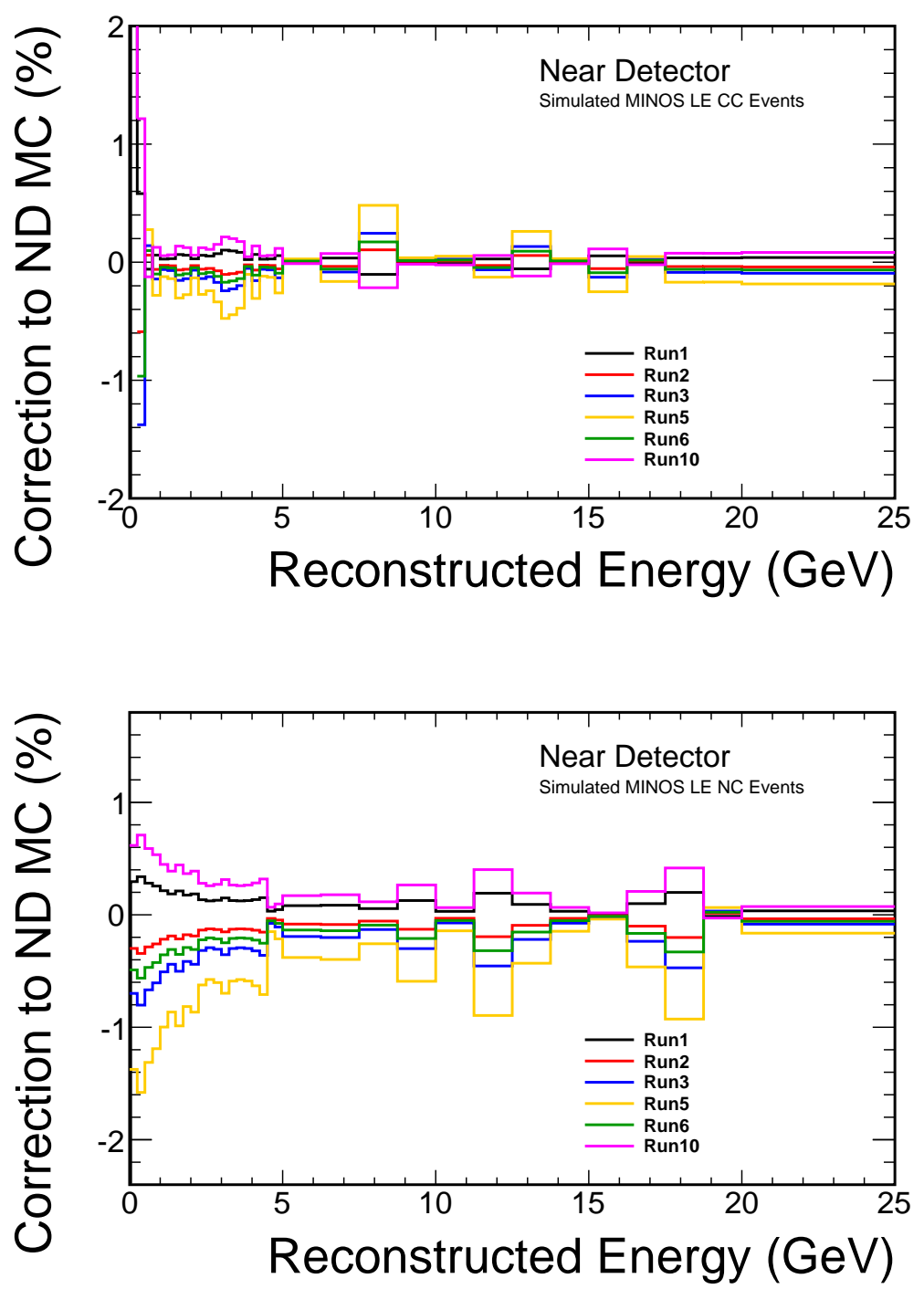

Figure 9.20: The total intensity corrections (in per cent) to be applied to the ND MC for charged- (top) and neutral-current (bottom) samples for all low-energy neutrino running. 
linearity that allows us to take a single $\mathrm{MC}$ ratio and scale it to correct for any intensity change.

To assess this assumption of linearity, figure 9.17 shows the ratios of the highintensity $\mathrm{MC}$ sample to of a number of MC samples of different intensities. It can be seen that the shape of all the ratios is similar, and the relative scale factor of each ratio is broadly in line with the intensity. To assess the linearity of this scale factor more quantitatively, in each bin of energy for both the NC and CC ratios from figure 9.17 a linear fit of the ratio as a function of intensity is performed. An example is shown in figure 9.21 for the $3 \mathrm{GeV}$ energy bin in the CC energy spectrum. The statistical error bands are derived from the MC samples and the nominal MC (Run 3 was used for these corrections, although this choice was arbitrary) is overlaid for comparison. A linear relationship is apparent for the MC ratios, however the data must also show a linear relationship against intensity. Figures 9.22 and 9.23 show the linear fit (MC points involved in the fit are removed for clarity) overlaid with the data from Run Period 3 across the energy bins 2, 4, 6, 10, 16 and $20 \mathrm{GeV}$. A good linear relationship is observed for both data and MC, thus justifying the assumption of linearity in the correction calculated above. 


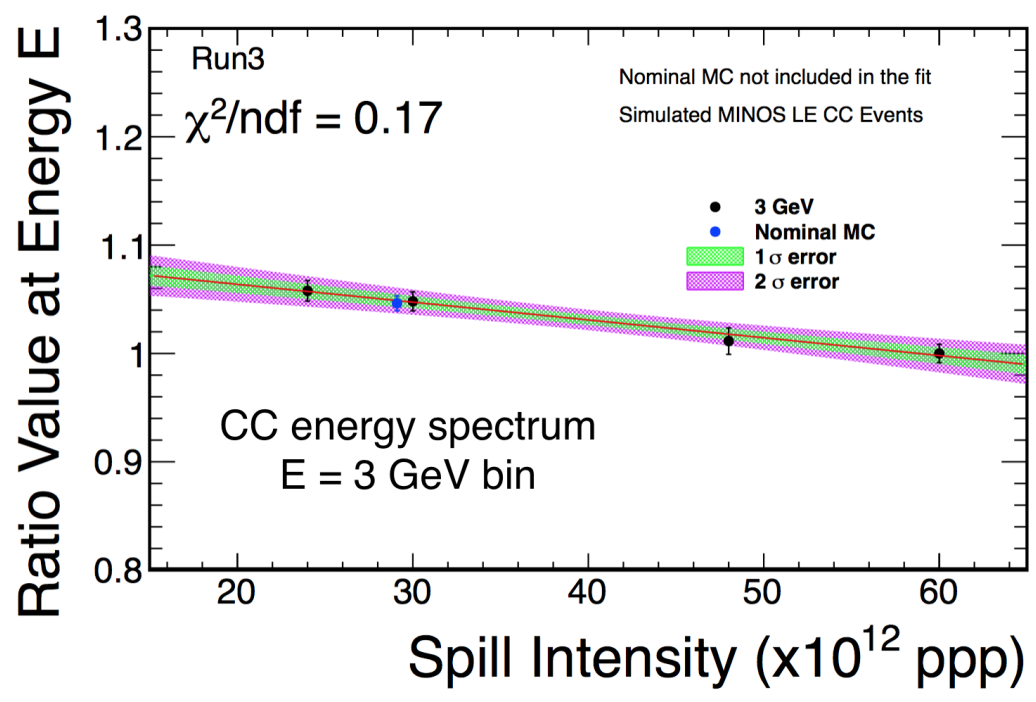

Figure 9.21: Linear fits to the Monte Carlo event rate as a function of intensity for Run Period 3 in the $3 \mathrm{GeV}$ energy bin. Note that the statistical error band is derived from the error on the black points. 


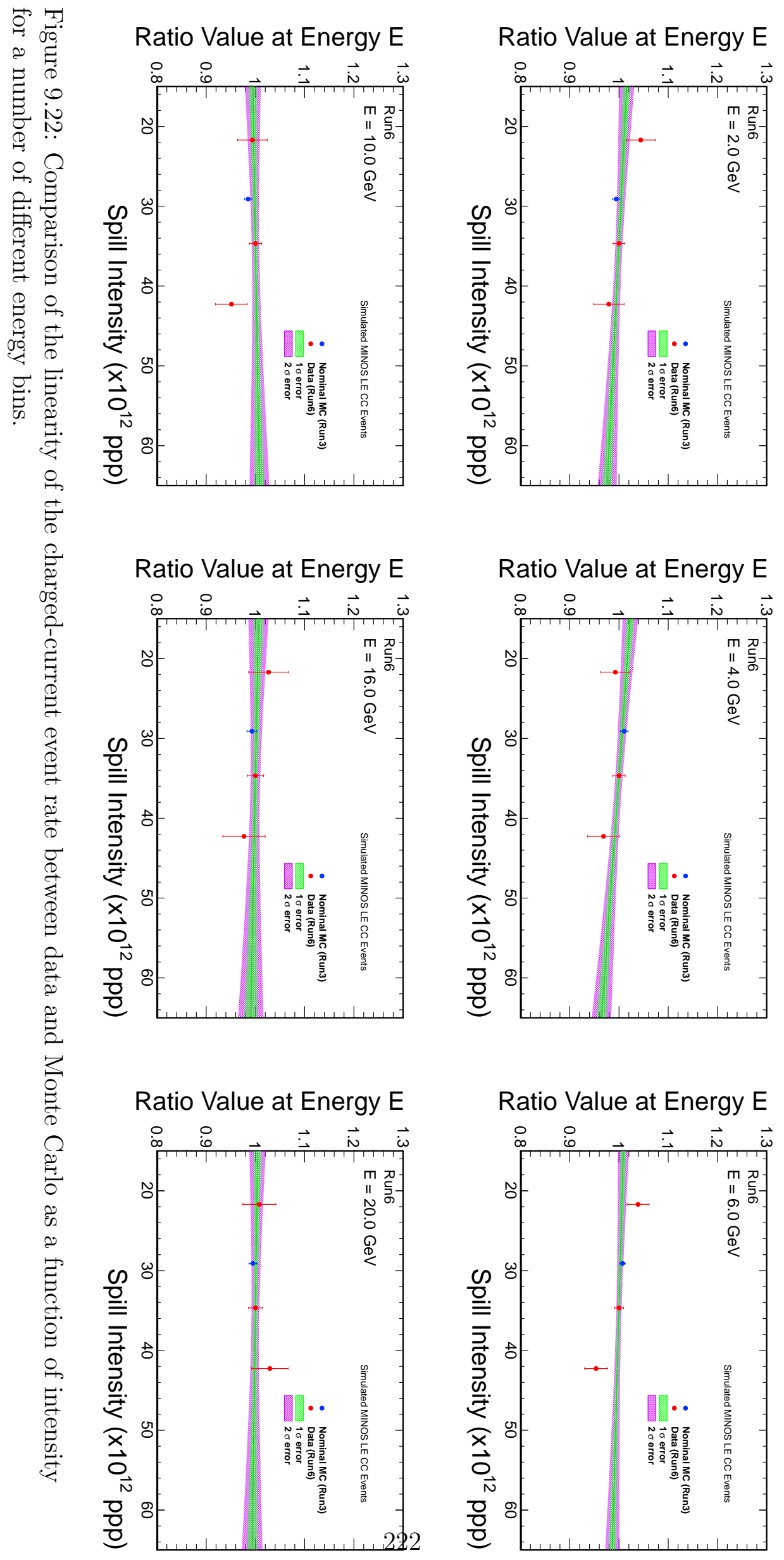



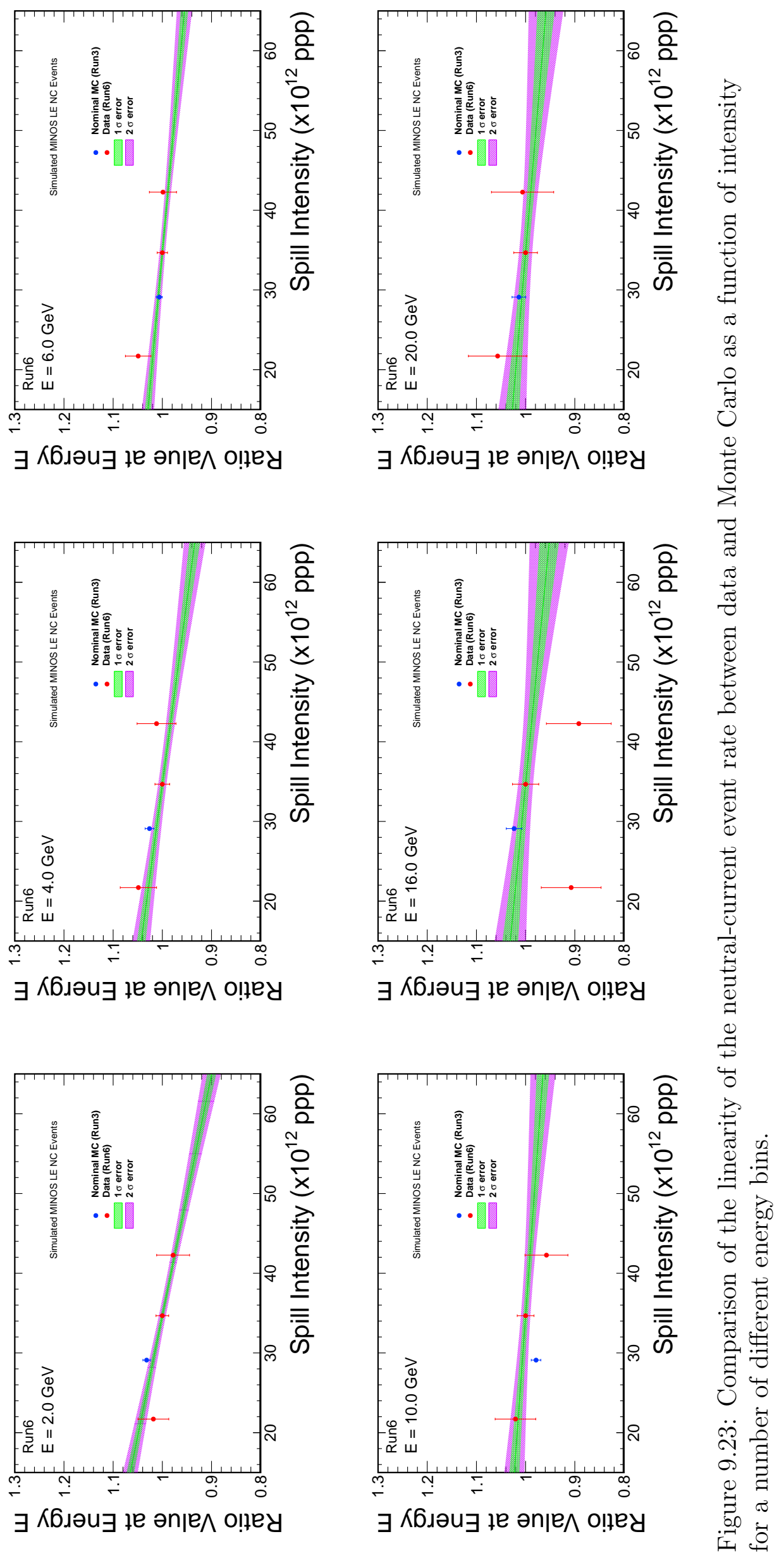


\section{Chapter 10}

\section{Conclusions and Future Outlook}

\subsection{Summary}

This thesis has used the MINOS data taken from 2005-2012 in the low-energy, $\nu_{\mu^{-}}$ dominated beam configuration. Using both neutrino events and the anti-neutrino background and assuming CPT conservation throughout, a sterile neutrino analysis has been performed looking for both the disappearance of charged current and neutral current events.

Using the standard MINOS extrapolation technique to produce a predicted FD energy spectrum, a model-independent approach was investigated by quantifying the amount of NC disappearance. Any deviations from the standard threeflavour model in the NC energy spectrum would be indicative of sterile neutrinos regardless of the model chosen (e.g. $3+1$ ). No obvious deviations can be seen in the data and the defined metric $R$ shows complete agreement within statistical and systematic errors with the null hypothesis.

This thesis then went on to look at the $3+1$ sterile neutrino model across a wide range of the parameter space $\left\{\Delta m_{41}^{2}, \sin ^{2}\left(\theta_{24}\right)\right\}$. This analysis explores parameter space in which oscillations can occur in both the Near and Far Detectors. Therefore a novel technique was developed by which both charged current 
and neutral current events are fit using the Far-over-Near energy spectra ratios directly. This technique allows ND oscillations to be incorporated. This thesis also reassess the associated systematic uncertainties and incorporates them into a covariance matrix to be used within the analysis.

The Far-over-Near fit saw no significant deviations from the three-flavour model and allowed this thesis to present strong constraints on the existence of sterile neutrinos spanning many orders of magnitude in $\Delta m_{41}^{2}$. The confidence limits computed were produced using the Feldman-Cousins unified approach and the non-Gaussianity of this analysis is discussed at length resulting in a large modification of the standard confidence limits one would obtain using Gaussian statistics.

\subsection{Future Outlook}

The MINOS+ experiment [80] began taking data in September 2013 and continued until the end of the summer 2016. MINOS+ was a continuation of the MINOS detectors but received a higher energy neutrino beam (the medium energy configuration). The neutrino beam in the MINOS+ era produced a neutrino energy spectrum with a peak at $\sim 6 \mathrm{GeV}$; combining this with the increased statistics will allow MINOS+ to improve on the sterile neutrino analysis presented in this thesis. Figure 10.1 shows the sensitivity of the MINOS+ data when combined with the MINOS data set used in this thesis.

Both MINOS and MINOS+ can distinguish between $\nu_{\mu}$ and $\bar{\nu}_{\mu}$ events due to the direction of the curved track from the muon in the final state and a dedicated sample with $\bar{\nu}_{\mu}$-enhanced beam beam is available for analysis. From this a sample of neutrinos or anti-neutrinos can be isolated. Future analyses can be performed, looking at only an anti-neutrino sample. Figure 10.2 shows the sensitivity of a $3+1$ sterile anti-neutrino analysis using an anti-neutrino sample from MINOS 


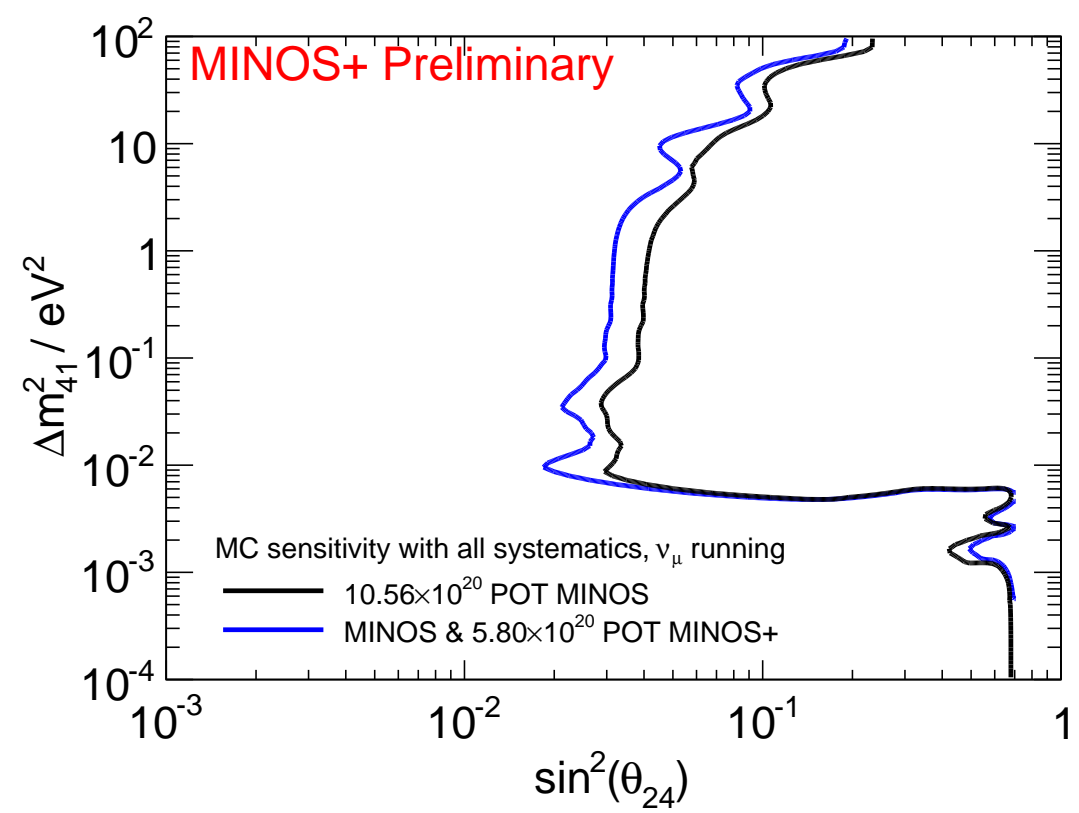

Figure 10.1: $90 \%$ C.L. sensitivity of the $\nu_{\mu}$-disappearance sterile neutrino search, comparing MINOS data to the combination of MINOS and MINOS+ data. The Feldman-Cousins unified approach has not been used to obtain these confidence intervals.

alone and from MINOS combined with possible MINOS $+\bar{\nu}_{\mu}$-enhanced running.

\subsection{Conclusion}

The new results presented in this thesis have contributed significantly to our understanding of sterile neutrinos. By looking at both charged-current and neutralcurrent events in the MINOS data sample no significant indication of sterile neutrinos has been found. A confidence limit has been placed in the parameter space $\left\{\Delta m_{41}^{2}, \sin ^{2} \theta_{24}\right\}$. By combining electron anti-neutrino disappearance search from Bugey-3 a confidence limit is placed on $\left\{\Delta m_{41}^{2}, \sin ^{2} 2 \theta_{\mu e}\right\}$, the parameter favoured by LSND and MiniBooNE. The results presented add to the tension between the anomalous results found in the neutrino appearance searches and the null results found by disappearance experiments. 


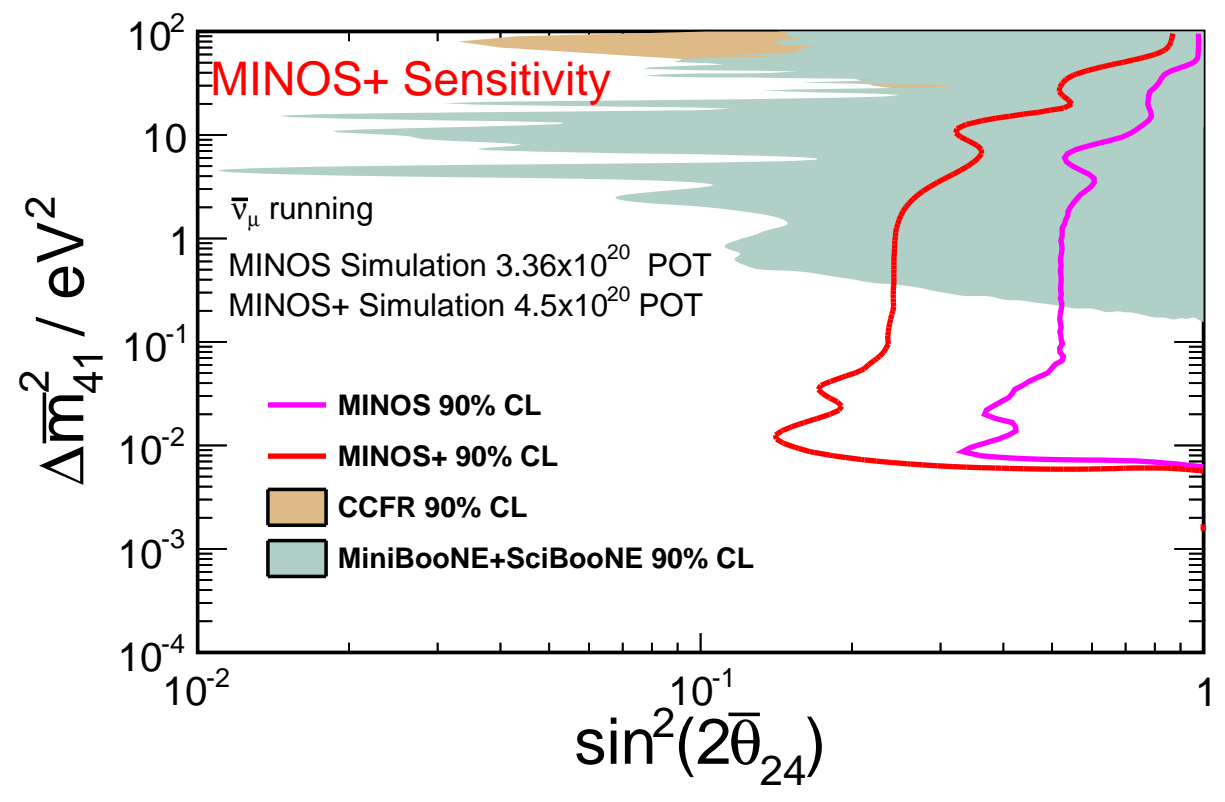

Figure 10.2: $90 \%$ C.L. sensitivity of the MINOS data to a sterile anti-neutrino search using $\bar{\nu}_{\mu}$ interactions. The sensitivity obtained through the addition of one year of MINOS $+\bar{\nu}_{\mu}$-enhanced running is also shown. The Feldman-Cousins unified approach has not been used to obtain these confidence intervals. The sensitivities are compared to previous $\bar{\nu}_{\mu}$-disappearance measurements from their experiments $[184,185]$. 
Appendices 


\section{Appendix A}

\section{Neutrinos Oscillations in a $3+1$}

\section{Model}

\section{A.1 PMNS Matrix for a 3+1 Model}

Mixing between neutrino mass states and flavour states is represented by a $4 \times 4$ rotation matrix, $U$ :

$$
U=\left(\begin{array}{cccc}
U_{e 1} & U_{e 2} & U_{e 3} & U_{e 4} \\
U_{\mu 1} & U_{\mu 2} & U_{\mu 3} & U_{\mu 4} \\
U_{\tau 1} & U_{\tau 2} & U_{\tau 3} & U_{\tau 4} \\
U_{s 1} & U_{s 2} & U_{s 3} & U_{s 4}
\end{array}\right)
$$

The PMNS matrix is a unitary matrix such that

$$
\sum_{k=1}^{4} U_{a k} U_{b k}^{*}=\delta_{a b}
$$


The matrix elements can be expressed as a function of the mixing angles and phases and are listed below using the notation $c_{i j}=\cos \theta_{i j}$ and $s_{i j}=\sin \theta_{i j}$ :

$$
\begin{aligned}
U_{e 1}= & c_{12} c_{13} c_{14}, \quad U_{e 2}=c_{13} c_{14} s_{12}, \quad U_{e 3}=c_{14} s_{13} e^{-i \delta_{13}}, \quad U_{e 4}=s_{14} e^{-i \delta_{14}}, \\
U_{\mu 1}= & c_{12}\left(-c_{24} s_{13} s_{23} e^{-i \delta_{13}}-c_{13} s_{14} s_{24} e^{-i\left(\delta_{24}-\delta_{14}\right)}\right)-c_{23} c_{24} s_{12}, \\
U_{\mu 2}= & c_{12} c_{23} c_{24}+s_{12}\left(-e^{i \delta_{13}} c_{24} s_{13} s_{23}-e^{-i\left(\delta_{24}-\delta_{14}\right)} c_{13} s_{14} s_{24}\right) \\
U_{\mu 3}= & c_{13} c_{24} s_{23}-s_{13} s_{14} s_{24} e^{-i\left(\delta_{13}-\delta_{14}+\delta_{24}\right)} \\
U_{\mu 4}= & c_{14} s_{24} e^{-i \delta_{24}}, \\
U_{\tau 1}= & -s_{12}\left(-c_{34} s_{23}-e^{i \delta_{24}} c_{23} s_{24} s_{34}\right) \\
& +c_{12}\left(-e^{i \delta_{14}} c_{13} c_{24} s_{14} s_{34}-s_{13} e^{i \delta_{13}}\left(c_{23} c_{34}-e^{i \delta_{24}} s_{23} s_{24} s_{34}\right)\right), \\
U_{\tau 2}= & -c_{12}\left(-c_{34} s_{23}-e^{i \delta_{24}} c_{23} s_{24} s_{34}\right) \\
& +s_{12}\left(-e^{i \delta_{14}} c_{13} c_{24} s_{14} s_{34}-s_{13} e^{i \delta_{13}}\left(c_{23} c_{34}-e^{i \delta_{24}} s_{23} s_{24} s_{34}\right)\right) \\
& +s_{12}\left(-e^{i \delta 24} c_{13} c_{24} c_{34} s_{14}-e^{i \delta_{13}} s_{13}\left(-e^{i \delta_{24}} c_{34} s_{23} s_{24}-c_{23} s_{34}\right)\right) \\
U_{s 3}= & -e^{-i\left(\delta_{13}-\delta_{14}\right)} c_{24} c_{34} s_{13} s_{14}+c_{13}\left(-e^{i \delta_{24}} c_{34} s_{23} s_{24}-c_{23} s_{34}\right) \\
U_{s 2}= & c_{12}\left(-e^{i \delta_{24}} c_{23} c_{34} s_{24}+s_{23} s_{34}\right) \\
U_{\tau 1}= & e^{-i\left(\delta_{13}-\delta_{14}\right)} c_{24} s_{13} s_{14} s_{34}+c_{13}\left(c_{23} c_{34}-e^{i \delta_{24}} s_{23} s_{24} s_{34}\right) \\
\left.U_{\tau 4} c_{13} c_{24} c_{34} s_{14}-e^{i \delta_{13}} s_{13}\left(-e^{i \delta_{24}} c_{34} s_{23} s_{24}-c_{23} s_{34}\right)\right) & -s_{12}\left(-e^{i \delta_{24}} c_{23} c_{34} s_{24}+s_{23} s_{34}\right) \\
&
\end{aligned}
$$




\section{A.2 Neutrino Oscillation Probabilities}

The general expression for a neutrino of initial flavour $\alpha$ oscillating within a vacuum into final flavour $\beta$ as derived in chapter 2 is

$$
\begin{aligned}
P\left(\nu_{\alpha} \rightarrow \nu_{\beta}\right)=P_{\alpha \beta}= & \delta_{\alpha \beta} \\
& +2 \sum_{i(>j)} \sum_{j} \mathfrak{I m}\left[U_{\beta j}^{*} U_{\beta i} U_{\alpha i}^{*} U_{\alpha j} \sin \left(\frac{\Delta m_{i j}^{2} L}{2 E}\right)\right] \\
& -4 \sum_{i(>j)} \sum_{j} \mathfrak{R e}\left[U_{\beta j}^{*} U_{\beta i} U_{\alpha i}^{*} U_{\alpha j} \sin ^{2}\left(\frac{\Delta m_{i j}^{2} L}{4 E}\right)\right] .
\end{aligned}
$$

Throughout this thesis in order to explain the sterile neutrino $3+1$ model several approximations are taken when presenting the mathematical expressions for the probability of neutrino oscillations. The actual analysis results use an exact form for the probabilities. These, however are long and non-trivial. For completeness they are presented in this appendix along with a simplified form using the approximation that the solar neutrino oscillation terms can be neglected.

\section{A.2.1 Muon neutrino survival: $P\left(\nu_{\mu} \rightarrow \nu_{\mu}\right)$}

For convenience one can redefine $\frac{\Delta m_{i j}^{2} L}{4 E}=\Delta_{i j}$. The first term (the Kronecker delta) becomes $\delta_{\mu \mu}=1$. The second term equates to zero as there is no imaginary component: $\sum_{i(>j)} \sum_{j} \mathfrak{I m}\left[U_{\mu j}^{*} U_{\mu i} U_{\mu i}^{*} U_{\mu j}\right]=0$. The muon survival probability in the $3+1$ model can now be expressed as

$$
P_{\mu \mu}=1-4 \sum_{i(>j)} \sum_{j}\left|U_{\mu j}\right|^{2}\left|U_{\mu i}\right|^{2} \sin ^{2} \Delta_{i j}
$$


where the summation can be expanded:

$$
\begin{aligned}
\sum_{i(>j)} \sum_{j}\left|U_{\mu j}\right|^{2}\left|U_{\mu i}\right|^{2} \sin ^{2} \Delta_{i j}= & \sum_{i>1}\left|U_{\mu 1}\right|^{2}\left|U_{\mu i}\right|^{2} \sin ^{2} \Delta_{i 1}+ \\
& \sum_{i>2}\left|U_{\mu 2}\right|^{2}\left|U_{\mu i}\right|^{2} \sin ^{2} \Delta_{i 2}+ \\
& \sum_{i>3}\left|U_{\mu 3}\right|^{2}\left|U_{\mu i}\right|^{2} \sin ^{2} \Delta_{i 3} .
\end{aligned}
$$

With all terms expanded the probability $P_{\mu \mu}$ is expressed, showing the dependence of all the mass-splitting terms as

$$
\begin{array}{r}
P_{\mu \mu}=1-4\left\{\left|U_{\mu 1}\right|^{2}\left|U_{\mu 2}\right|^{2} \sin ^{2} \Delta_{21}+\left|U_{\mu 1}\right|^{2}\left|U_{\mu 3}\right|^{2} \sin ^{2} \Delta_{31}+\right. \\
\left|U_{\mu 1}\right|^{2}\left|U_{\mu 4}\right|^{2} \sin ^{2} \Delta_{41}+\left|U_{\mu 2}\right|^{2}\left|U_{\mu 3}\right|^{2} \sin ^{2} \Delta_{32}+ \\
\left.\left|U_{\mu 2}\right|^{2}\left|U_{\mu 4}\right|^{2} \sin ^{2} \Delta_{42}+\left|U_{\mu 3}\right|^{2}\left|U_{\mu 4}\right|^{2} \sin ^{2} \Delta_{43}\right\} .
\end{array}
$$

From here the approximation can be made that all solar terms are neglected due to the $L / E$ probed by the MINOS detectors, such that $\Delta_{41} \cong \Delta_{42}$ and $\Delta_{32} \cong \Delta_{31}$. This simplifies the expression of $P_{\mu \mu}$ to

$$
\begin{aligned}
P_{\mu \mu} \approx & -4\left(1-\left|U_{\mu 3}\right|^{2}-\left|U_{\mu 4}\right|^{2}\right)\left|U_{\mu 3}\right|^{2} \sin ^{2} \Delta_{31} \\
& -4\left(1-\left|U_{\mu 3}\right|^{2}-\left|U_{\mu 4}\right|^{2}\right)\left|U_{\mu 4}\right|^{2} \sin ^{2} \Delta_{41} \\
& -4\left|U_{\mu 3}\right|^{2}\left|U_{\mu 4}\right|^{2} \sin ^{2} \Delta_{43},
\end{aligned}
$$

where the unitarity constraint $\sum_{k=1}^{4}\left|U_{\mu k}\right|^{2}=1$ has been used such that the muon neutrino survival probability can be expressed in terms of $\left|U_{\mu 3}\right|^{2}$ and $\left|U_{\mu 4}\right|^{2}$. 


\section{A.2.2 Sterile neutrino appearance: $P\left(\nu_{\mu} \rightarrow \nu_{s}\right)$}

The following is the expression for sterile neutrino appearance where $\delta_{\mu s}=0$ :

$$
\begin{aligned}
P_{\mu s}= & +2 \sum_{i(>j)} \sum_{j} \mathfrak{I m}\left[U_{s j}^{*} U_{s i} U_{\mu i}^{*} U_{\mu j}\right] \sin 2 \Delta_{i j} \\
& -4 \sum_{i(>j)} \sum_{j} \mathfrak{R e}\left[U_{s j}^{*} U_{s i} U_{\mu i}^{*} U_{\mu j}\right] \sin ^{2} \Delta_{i j} .
\end{aligned}
$$

\section{A.2.2.1 The $\mathfrak{I m}$ term}

First we consider the first term in equation A.10:

$$
2 \sum_{i(>j)} \sum_{j} \mathfrak{I m}\left[U_{s j}^{*} U_{s i} U_{\mu i}^{*} U_{\mu j}\right] \sin 2 \Delta_{i j}
$$

which can be expanded and expressed as

$$
\begin{gathered}
2\left[\mathfrak{I m}\left[U_{s 1}^{*} U_{s 2} U_{\mu 2}^{*} U_{\mu 1}\right] \sin 2 \Delta_{21}+\mathfrak{I m}\left[U_{s 1}^{*} U_{s 3} U_{\mu 3}^{*} U_{\mu 1}\right] \sin 2 \Delta_{31}+\right. \\
\mathfrak{I m}\left[U_{s 1}^{*} U_{s 4} U_{\mu 4}^{*} U_{\mu 1}\right] \sin 2 \Delta_{41}+\mathfrak{I m}\left[U_{s 2}^{*} U_{s 3} U_{\mu 3}^{*} U_{\mu 2}\right] \sin 2 \Delta_{32}+ \\
\left.\mathfrak{I m}\left[U_{s 2}^{*} U_{s 4} U_{\mu 4}^{*} U_{\mu 2}\right] \sin 2 \Delta_{42}+\mathfrak{I m}\left[U_{s 3}^{*} U_{s 4} U_{\mu 4}^{*} U_{\mu 3}\right] \sin 2 \Delta_{43}\right] .
\end{gathered}
$$

To further simplify this expression one can assume that the solar neutrino oscillation terms are negligible such that $\Delta_{42} \cong \Delta_{41}$ and $\Delta_{32} \cong \Delta_{31}$, resulting in

$$
\begin{aligned}
& 2\left[\mathfrak{I m}\left[U_{s 1}^{*} U_{s 3} U_{\mu 3}^{*} U_{\mu 1}+U_{s 2}^{*} U_{s 3} U_{\mu 3}^{*} U_{\mu 2}\right] \sin 2 \Delta_{31}+\right. \\
& \mathfrak{I m}\left[U_{s 1}^{*} U_{s 4} U_{\mu 4}^{*} U_{\mu 1}+U_{s 2}^{*} U_{s 4} U_{\mu 4}^{*} U_{\mu 2}\right] \sin 2 \Delta_{41}+ \\
& \left.\quad \mathfrak{I m}\left[U_{s 3}^{*} U_{s 4} U_{\mu 4}^{*} U_{\mu 3}\right] \sin 2 \Delta_{43}\right] .
\end{aligned}
$$


Using the following unitarity relation,

$$
U_{s 1}^{*} U_{\mu 1}+U_{s 2}^{*} U_{\mu 2}+U_{s 3}^{*} U_{\mu 3}+U_{s 4}^{*} U_{\mu 4}=0
$$

one can express everything in terms of the matrix elements $U_{s 3}, U_{s 4}, U_{\mu 3}$ and $U_{\mu 4}$ to give

$$
\begin{gathered}
-2\left[\mathfrak{I m}\left[U_{s 3} U_{\mu 3}^{*}\left(U_{s 3}^{*} U_{\mu 3}+U_{s 4}^{*} U_{\mu 4}\right)\right] \sin 2 \Delta_{31}+\right. \\
\mathfrak{I m}\left[U_{s 4} U_{\mu 4}^{*}\left(U_{s 3}^{*} U_{\mu 3}+U_{s 4}^{*} U_{\mu 4}\right)\right] \sin 2 \Delta_{41}- \\
\left.\mathfrak{I m}\left[U_{s 3}^{*} U_{s 4} U_{\mu 4}^{*} U_{\mu 3}\right] \sin 2 \Delta_{43}\right] .
\end{gathered}
$$

Several terms are zero due to $\mathfrak{I m}\left[U_{\alpha i} U_{\alpha i}^{*}\right]=0$. Like terms can be collected and, by using the following relation,

$$
\mathfrak{I m}\left[U_{s 3} U_{\mu 3}^{*} U_{s 4}^{*} U_{\mu 4}\right]=-\mathfrak{I m}\left[U_{s 3}^{*} U_{\mu 3} U_{s 4} U_{\mu 4}^{*}\right]
$$

the final expression for the imaginary term is

$$
\approx 2 \mathfrak{I m}\left[U_{\mu 3} U_{s 3}^{*} U_{\mu 4}^{*} U_{s 4}\right]\left(\sin 2 \Delta_{31}-\sin 2 \Delta_{41}+\sin 2 \Delta_{43}\right) .
$$

\section{A.2.2.2 The $\mathfrak{R e}$ term}

Next, the real component term is considered:

$$
-4 \sum_{i(>j)} \sum_{j} \mathfrak{R e}\left[U_{s j}^{*} U_{s i} U_{\mu i}^{*} U_{\mu j}\right] \sin ^{2} \Delta_{i j}
$$

The procedure is similar to that of evaluating the imaginary term. The same approximation is used that the solar neutrino oscillation terms are negligible such that $\Delta_{42} \cong \Delta_{41}$ and $\Delta_{32} \cong \Delta_{31}$. However, a different relation is used for the 
PMNS matrix elements:

$$
\mathfrak{R e}\left[U_{s 3} U_{\mu 3}^{*} U_{s 4}^{*} U_{\mu 4}\right]=\mathfrak{R e}\left[U_{s 3}^{*} U_{\mu 3} U_{s 4} U_{\mu 4}^{*}\right] .
$$

This results in an expression for the real term:

$$
\begin{aligned}
& 4\left|U_{s 3}\right|^{2}\left|U_{\mu 3}\right|^{2} \sin ^{2} \Delta_{31}+4\left|U_{s 4}\right|^{2}\left|U_{\mu 4}\right|^{2} \sin ^{2} \Delta_{41} \\
& +4 \Re \mathfrak{R e}\left[U_{\mu 3} U_{s 3}^{*} U_{\mu 4}^{*} U_{s 4}\right]\left(\sin ^{2} \Delta_{41}-\sin ^{2} \Delta_{43}+\sin ^{2} \Delta_{31}\right) .
\end{aligned}
$$

\section{A.2.2.3 The total expression}

Using the expressions derived in equations A.7 and A.8, the probability for sterile neutrino appearance under the assumption that the $L / E$ of the experiment is such that the solar neutrino terms can be considered negligible is

$$
\begin{aligned}
P_{\mu s} \approx & 4\left|U_{s 3}\right|^{2}\left|U_{\mu 3}\right|^{2} \sin ^{2} \Delta_{31}+4\left|U_{s 4}\right|^{2}\left|U_{\mu 4}\right|^{2} \sin ^{2} \Delta_{41} \\
& +4 \mathfrak{R e}\left[U_{\mu 3} U_{s 3}^{*} U_{\mu 4}^{*} U_{s 4}\right]\left(\sin ^{2} \Delta_{31}+\sin ^{2} \Delta_{41}-\sin ^{2} \Delta_{43}\right) \\
& +2 \mathfrak{I m}\left[U_{\mu 3} U_{s 3}^{*} U_{\mu 4}^{*} U_{s 4}\right]\left(\sin 2 \Delta_{31}-\sin 2 \Delta_{41}+\sin 2 \Delta_{43}\right) .
\end{aligned}
$$

\section{A.2.3 Electron neutrino appearance: $P\left(\nu_{\mu} \rightarrow \nu_{e}\right)$}

The following is the expression for electron neutrino appearance where $\delta_{\mu e}=0$ :

$$
\begin{aligned}
P_{\mu e}= & +2 \sum_{i(>j)} \sum_{j} \mathfrak{I m}\left[U_{e j}^{*} U_{e i} U_{\mu i}^{*} U_{\mu j}\right] \sin 2 \Delta_{i j} \\
& -4 \sum_{i(>j)} \sum_{j} \mathfrak{R e}\left[U_{e j}^{*} U_{e i} U_{\mu i}^{*} U_{\mu j}\right] \sin ^{2} \Delta_{i j} .
\end{aligned}
$$


Similar to the previous derivations, the imaginary term is considered first. The summation is expanded:

$$
\begin{gathered}
2\left[\mathfrak{I m}\left[U_{e 1}^{*} U_{e 2} U_{\mu 2}^{*} U_{\mu 1}\right] \sin 2 \Delta_{21}+\mathfrak{I m}\left[U_{e 1}^{*} U_{e 3} U_{\mu 3}^{*} U_{\mu 1}\right] \sin 2 \Delta_{31}+\right. \\
\mathfrak{I m}\left[U_{e 1}^{*} U_{e 4} U_{\mu 4}^{*} U_{\mu 1}\right] \sin 2 \Delta_{41}+\mathfrak{I m}\left[U_{e 2}^{*} U_{e 3} U_{\mu 3}^{*} U_{\mu 2}\right] \sin 2 \Delta_{32}+ \\
\left.\mathfrak{I m}\left[U_{e 2}^{*} U_{e 4} U_{\mu 4}^{*} U_{\mu 2}\right] \sin 2 \Delta_{42}+\mathfrak{I m}\left[U_{e 3}^{*} U_{e 4} U_{\mu 4}^{*} U_{\mu 3}\right] \sin 2 \Delta_{43}\right]
\end{gathered}
$$

To relate this to the expression used for electron neutrino appearance for shortbaseline experiments (LSND and MiniBooNE) the approximation is taken that the sterile neutrino mass state, $\nu_{4}$, is much heavier than the active neutrino mass states, $\nu_{1}, \nu_{2}$ and $\nu_{3}$. As such the approximation is made such that $\Delta_{41} \cong \Delta_{42} \cong$ $\Delta_{43}$ with all other mass splitting terms being considered negligible. This simplifies the expression:

$$
2 \mathfrak{I m}\left[U_{e 4} U_{\mu 4}^{*}\left(U_{e 1}^{*} U_{\mu 1}+U_{e 2}^{*} U_{\mu 2}+U_{e 3}^{*} U_{\mu 3}\right)\right] \sin 2 \Delta_{41}
$$

which can be further simplified using the unitary relation

$$
U_{e 1}^{*} U_{\mu 1}+U_{e 2}^{*} U_{\mu 2}+U_{e 3}^{*} U_{\mu 3}+U_{e 4}^{*} U_{\mu 4}=0
$$

such that the imaginary term can be expressed as

$$
-2 \mathfrak{I m}\left[\left|U_{e 4}\right|^{2}\left|U_{\mu 4}\right|^{2}\right] \sin 2 \Delta_{41}=0
$$

The same procedure is then performed on the real term. Skipping the algebra, the expression becomes

$$
4 \mathfrak{R e}\left[\left|U_{e 4}\right|^{2}\left|U_{\mu 4}\right|^{2}\right] \sin ^{2} \Delta_{41}=4\left|U_{e 4}\right|^{2}\left|U_{\mu 4}\right|^{2} \sin ^{2} \Delta_{41}
$$


The final expression for the electron neutrino appearance probability for a large mass splitting between the sterile and active neutrinos can therefore be written as

$$
P_{\mu e} \approx 4\left|U_{e 4}\right|^{2}\left|U_{\mu 4}\right|^{2} \sin ^{2} \Delta_{41}=\sin ^{2} 2 \theta_{\mu e} \sin ^{2} \Delta_{41}
$$

where $\sin ^{2} 2 \theta_{\mu e}=4\left|U_{e 4}\right|^{2}\left|U_{\mu 4}\right|^{2}$. 


\section{Bibliography}

[1] W. Pauli, Letter, addressed to participents of Tübingen conference on radioactivity 1930, Available from the CERN Document server: http://cdsweb.cern.ch/record/83282.

[2] J. Chadwick, The intensity distribution in the magnetic spectrum of beta particles from radium (B + C), Verh. Phys. Gesell. 16, 383 (1914).

[3] J. Chadwick, The Existence of a Neutron, Proc. Roy. Soc. Lond. A136, 692 (1932).

[4] J. Chadwick, Possible Existence of a Neutron, Nature 129, 312 (1932).

[5] F. Perrin, Possibilité d'émission de particules neutres de masse intrinsèque nulle dans les radiactivités $\beta$. (The possibiliy of the emission of neutral particles of zero mass during $\beta$ decay.), Comptes Rendus des Séances de l'Académie des Sciences 197, 1625 (1933).

[6] E. Fermi, Versuch einer Theorie der $\beta$-Strahlen. I. (Attempt at a Theory of $\beta$-Rays. I.), Z. Phys. 88, 161 (1934).

[7] F. L. Wilson, Fermi's Theory of Beta Decay, Am. J. Phys. 36, 1150 (1968).

[8] C. Cowan, F. Reines, F. Harrison, E. Anderson, and F. Hayes, Large Liquid Scintillation Detectors, Phys. Rev. 90, 493 (1953).

[9] F. Reines and C. Cowan, Detection of the Free Neutrino, Phys. Rev. 92, $830(1953)$. 
[10] F. Reines and C. Cowan, The Neutrino, Nature 178, 446 (1956).

[11] F. Reines and C. Cowan, A Proposed Experiment to Detect the Free Neutrino, Phys. Rev. 90, 492 (1953).

[12] F. Reines, C. Cowan, F. Harrison, A. McGuire, and H. Kruse, Detection of the Free Antineutrino, Phys. Rev. 117, 159 (1960).

[13] B. Pontecorvo, Electron and Muon Neutrinos, Sov. Phys. JETP 10, 1236 (1960).

[14] C. D. Anderson and S. H. Neddermeyer, Cloud Chamber Observations of Cosmic Rays at 4300 Meters Elevation and Near Sea-Level, Phys. Rev. 50, 263 (1936).

[15] S. H. Neddermeyer and C. D. Anderson, Note on the Nature of Cosmic-Ray Particles, Phys. Rev. 51, 884 (1937).

[16] G. Danby et al., Observation of High-Energy Neutrino Reactions and the Existence of Two Kinds of Neutrinos, Phys. Rev. Lett. 9, 36 (1962).

[17] M. L. Perl et al., Evidence for Anomalous Lepton Production in $e^{+}-e^{-}$ Annihilation, Phys. Rev. Lett. 35, 1489 (1975).

[18] SLD Electroweak Group, DELPHI, ALEPH, SLD, SLD Heavy Flavour Group, OPAL, LEP Electroweak Working Group, L3 Collaboration: S. Schael et al., Precision electroweak measurements on the $Z$ resonance, Phys. Rept. 427, 257 (2006).

[19] DONUT Collaboration: K. Kodama et al., Observation of Tau Neutrino Interactions, Phys. Lett. B504, 218 (2001).

[20] T. D. Lee and C. N. Yang, Question of Parity Conservation in Weak Interactions, Phys. Rev. 104, 254 (1956). 
[21] C. S. Wu, E. Ambler, R. W. Hayward, D. D. Hoppes, and R. P. Hudson, Experimental Test of Parity Conservation in Beta Decay, Phys. Rev. 105, 1413 (1957).

[22] M. Goldhaber, L. Grodzins, and A. W. Sunyar, Helicity of Neutrinos, Phys. Rev. 109, 1015 (1958).

[23] B. Pontecorvo, Mesonium and anti-mesonium, Sov. Phys. JETP 6, 429 (1957).

[24] B. Pontecorvo, Inverse beta processes and nonconservation of lepton charge, Sov. Phys. JETP 7, 172 (1958).

[25] Z. Maki, M. Nakagawa, and S. Sakata, Remarks on the unified model of elementary particles, Prog. Theor. Phys. 28, 870 (1962).

[26] R. Davis, D. S. Harmer, and K. C. Hoffman, Search for neutrinos from the sun, Phys. Rev. Lett. 20, 1205 (1968).

[27] J. N. Bahcall, N. A. Bahcall, and G. Shaviv, Present status of the theoretical predictions for the ${ }^{37} \mathrm{Cl}$ solar neutrino experiment, Phys. Rev. Lett. 20, 1209 (1968).

[28] Particle Data Group Collaboration: K. A. Olive et al., Review of Particle Physics, Chin. Phys. C38, 090001 (2014).

[29] J. Bahcall, Neutrino Astrophysics (Cambridge University Press, 1989).

[30] J. N. Bahcall and M. H. Pinsonneault, What do we (not) know theoretically about solar neutrino fluxes?, Phys. Rev. Lett. 92, 121301 (2004).

[31] J. N. Bahcall, A. M. Serenelli, and S. Basu, New solar opacities, abundances, helioseismology, and neutrino fluxes, Astrophys. J. 621, L85 (2005). 
[32] K. Lande et al., Solar neutrino observations with the Homestake ${ }^{37} \mathrm{Cl}$ detector, AIP Conf. Proc. 243, 1122 (1992).

[33] Kamiokande-II Collaboration: K. S. Hirata et al., Observation of ${ }^{8}$ B solar neutrinos in the Kamiokande-II detector, Phys. Rev. Lett. 63, 16 (1989).

[34] A. I. Abazov et al., Search for neutrinos from the Sun using the reaction ${ }^{71} \mathrm{Ga}\left(\nu_{e}, e^{-}\right){ }^{71} \mathrm{Ge}$, Phys. Rev. Lett. 67, 3332 (1991).

[35] GALLEX Collaboration: P. Anselmann et al., Solar neutrinos observed by GALLEX at Gran Sasso., Phys. Lett. B285, 376 (1992).

[36] SAGE Collaboration: J. Abdurashitov et al., Measurement of the Solar Neutrino Capture Rate by the Russian-American Gallium Solar Neutrino Experiment During One Half of the 22-Year Cycle of Solar Activity, ZhETP 122, 1 (2002).

[37] GALLEX Collaboration: W. Hampel et al., GALLEX Solar Neutrino Observations: Results for GALLEX IV, Phys. Lett. B447, 127 (1999).

[38] GNO Collaboration: M. Altmann et al., Complete results for five years of GNO solar neutrino observations, Phys. Lett. B616, 174 (2005).

[39] Y. Fukuda et al., Solar Neutrino Data Covering Solar Cycle 22, Phys. Rev. Lett. 77, 1683 (1996).

[40] K. S. Hirata et al., Real-time, directional measurement of ${ }^{8} \mathrm{~B}$ solar neutrinos in the Kamiokande II detector, Phys. Rev. D44, 2241 (1991).

[41] Super-Kamiokande Collaboration Collaboration: J. Hosaka et al., Solar neutrino measurements in Super-Kamiokande-I, Phys. Rev. D73, 112001 (2006).

[42] SNO Collaboration: B. Aharmim et al., Electron energy spectra, fluxes, and day-night asymmetries of ${ }^{8} \mathrm{~B}$ solar neutrinos from measurements with $\mathrm{NaCl}$ 
dissolved in the heavy-water detector at the Sudbury Neutrino Observatory, Phys. Rev. C72, 055502 (2005).

[43] Kamiokande-II Collaboration: K. S. Hirata et al., Experimental Study of the Atmospheric Neutrino Flux, Phys. Lett. B205, 416 (1988).

[44] R. Becker-Szendy et al., Electron-and muon-neutrino content of the atmospheric flux, Phys. Rev. D46, 3720 (1992).

[45] W. W. M. Allison et al., Measurement of the atmospheric neutrino flavour composition in Soudan-2, Phys. Lett. B391, 491 (1997).

[46] KamLAND Collaboration: K. Eguchi et al., First results from KamLAND: Evidence for reactor anti-neutrino disappearance, Phys. Rev. Lett. 90, $021802(2003)$.

[47] K2K Collaboration: M. H. Ahn et al., Measurement of Neutrino Oscillation by the K2K Experiment, Phys. Rev. D74, 072003 (2006).

[48] MINOS Collaboration: D. G. Michael et al., Observation of muon neutrino disappearance with the MINOS detectors and the NuMI neutrino beam, Phys. Rev. Lett. 97, 191801 (2006).

[49] MINOS Collaboration: P. Adamson et al., New constraints on muonneutrino to electron-neutrino transitions in MINOS, Phys. Rev. D82, $051102(2010)$.

[50] N. Agafonova et al., The detection of neutrino interactions in the emulsion/lead target of the OPERA experiment, JINST 4, P06020 (2009).

[51] K. Matsuoka et al., Design and performance of the muon monitor for the T2K neutrino oscillation experiment, Nucl. Instrum. Meth. A624, 591 $(2010)$ 
[52] NO $\nu$ A Collaboration: P. Adamson et al., First measurement of electron neutrino appearance in NO $\nu$ A, Phys. Rev. Lett. 116, 151806 (2016).

[53] NO $\nu$ A Collaboration: P. Adamson et al., First measurement of muon-

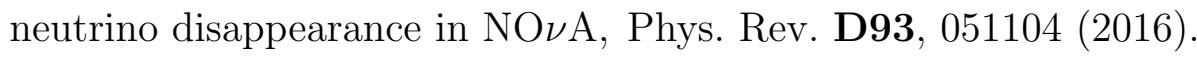

[54] S. E. Kopp, Accelerator-based neutrino beams, Phys. Rept. 439, 101 (2007).

[55] B. Kayser, On the quantum mechanics of neutrino oscillation, Phys. Rev. D24, 110 (1981).

[56] C. Giunti, C. W. Kim, and U. W. Lee, When do neutrinos really oscillate? Quantum mechanics of neutrino oscillations, Phys. Rev. D44, 3635 (1991).

[57] L. Wolfenstein, Neutrino Oscillations in Matter, Phys. Rev. D17, 2369 (1978).

[58] S. P. Mikheev and A. Yu. Smirnov, Resonance Amplification of Oscillations in Matter and Spectroscopy of Solar Neutrinos, Sov. J. Nucl. Phys. 42, 913 (1985).

[59] A. Yu. Smirnov, The MSW effect and matter effects in neutrino oscillations, Phys. Scripta T121, 57 (2005).

[60] F. Capozzi et al., Status of three-neutrino oscillation parameters, circa 2013, Phys. Rev. D 89, 093018 (2014).

[61] V. N. Gribov and B. Pontecorvo, Neutrino astronomy and lepton charge, Phys. Lett. B28, 493 (1969).

[62] K. N. Abazajian et al., Light Sterile Neutrinos: A White Paper, (2012), arXiv:1204.5379. 
[63] LSND Collaboration: A. Aguilar et al., Evidence for neutrino oscillations from the observation of $\bar{\nu}_{e}$ appearance in a $\bar{\nu}_{\mu}$ beam, Phys. Rev. D64, 112007 (2001).

[64] MiniBooNE Collaboration: A. A. Aguilar-Arevalo et al., Improved Search for $\bar{\nu}_{\mu} \rightarrow \bar{\nu}_{e}$ Oscillations in the MiniBooNE Experiment, Phys. Rev. Lett. 110, $161801(2013)$.

[65] P. Vogel, Analysis of the anti-neutrino capture on protons, Phys. Rev. D29, 1918 (1984).

[66] G. Mention et al., Reactor Anti-neutrino Anomaly, Phys. Rev. D83, 073006 (2011).

[67] T. A. Mueller et al., Improved Predictions of Reactor Antineutrino Spectra, Phys. Rev. C83, 054615 (2011).

[68] D. Abdurashitov et al., The Russian-American gallium experiment (SAGE) Cr neutrino source measurement, Phys. Rev. Lett. 77, 4708 (1996).

[69] J. N. Abdurashitov et al., Measurement of the response of a Ga solar neutrino experiment to neutrinos from an Ar-37 source, Phys. Rev. C73, $045805(2006)$.

[70] GALLEX Collaboration: W. Hampel et al., Final results of the ${ }^{51} \mathrm{Cr}$ neutrino source experiments in GALLEX, Phys. Lett. B420, 114 (1998).

[71] F. Kaether, W. Hampel, G. Heusser, J. Kiko, and T. Kirsten, Reanalysis of the GALLEX solar neutrino flux and source experiments, Phys. Lett. B685, 47 (2010).

[72] C. Giunti and M. Laveder, Statistical Significance of the Gallium Anomaly, Phys. Rev. C83, 065504 (2011). 
[73] B. Armbruster et al., Search for $\bar{\nu}_{\mu} \rightarrow \bar{\nu}_{e}$ oscillations with KARMEN2, Nucl. Phys. A663, 803 (2000).

[74] ICARUS Collaboration: M. Antonello et al., Search for anomalies in the $\nu_{e}$ appearance from a $\nu_{\mu}$ beam, Eur. Phys. J. C73, 2599 (2013).

[75] D. Ayres et al., P-875: A Long Baseline Neutrino Oscillation Experiment at Fermilab, MINOS Proposal, NuMI-L-63.

[76] MINOS Collaboration: D. G. Michael et al., The magnetized steel and scintillator calorimeters of the MINOS experiment, Nucl. Instrum. Meth. A596, 190 (2008).

[77] P. Adamson et al., The NuMI Neutrino Beam, Nucl. Instrum. Meth. A806, $279(2016)$.

[78] MINOS Collaboration: P. Adamson et al., Measurements of atmospheric neutrinos and antineutrinos in the MINOS Far Detector, Phys. Rev. D86, 052007 (2012).

[79] MINOS Collaboration: P. Adamson et al., Combined analysis of $\nu_{\mu}$ disappearance and $\nu_{\mu} \rightarrow \nu_{e}$ appearance in MINOS using accelerator and atmospheric neutrinos, Phys. Rev. Lett. 112, 191801 (2014).

[80] MINOS+ Collaboration: G. Tzanankos et al., MINOS+: a Proposal to FNAL to run MINOS with the medium energy NuMI beam, (2011), FERMILAB-PROPOSAL-1016.

[81] MINOS Collaboration: P. Adamson et al., Search for flavor-changing nonstandard neutrino interactions by MINOS, Phys. Rev. D88, 072011 (2013).

[82] N. Arkani-Hamed, S. Dimopoulos, and G. R. Dvali, The Hierarchy problem and new dimensions at a millimeter, Phys. Lett. B429, 263 (1998). 
[83] K. Anderson et al., The NuMI Facility Technical Design Report, (1998), FERMILAB-DESIGN-1998-01.

[84] S. Shukla et al., Slip stacking for the Fermilab luminosity upgrade, Conf. Proc. C970512, 144 (1997).

[85] A. Radovic, Measuring the Disappearance of Muon Neutrinos with the MINOS Detector., PhD thesis U. Coll. London 2013, FERMILAB-THESIS2013-24.

[86] MINOS Collaboration: R. K. Plunkett and J. A. Thomas, Proposal for a Cosmic Ray Veto Shield for the MINOS Far Detector, (2002), FERMILABPROPOSAL-0934.

[87] MINOS Collaboration: I. Ambats et al., The MINOS Detectors Technical Design Report, (1998), FERMILAB-DESIGN-1998-02.

[88] MINOS Collaboration: J. K. Nelson and J. Kilmer, MINOS far-detector coil design, (1999), FERMILAB-TM-2408-E.

[89] MINOS Collaboration: J. K. Nelson and J. Kilmer, MINOS near-detector coil design, (1999), FERMILAB-TM-2407.

[90] MINOS Collaboration: P. Adamson et al., First direct observation of muon antineutrino disappearance, Phys. Rev. Lett. 107, 021801 (2011).

[91] MINOS Collaboration: P. Adamson et al., Improved search for muonneutrino to electron-neutrino oscillations in MINOS, Phys. Rev. Lett. 107, 181802 (2011).

[92] S. Avvakumov et al., Spontaneous light emission from fibers in MINOS, Nucl. Instrum. Meth. A545, 145 (2005). 
[93] J. Oliver, N. Felt, G. Feldman, A. Lebedev, and R. Lee, Design and performance of the readout system of the MINOS Far Detector, IEEE Trans. Nucl. Sci. 51, 2193 (2004).

[94] T. Cundiff et al., The MINOS near detector front end electronics, IEEE Trans. Nucl. Sci. 53, 1347 (2006).

[95] MINOS Collaboration: A. Cabrera et al., Comparisons of the MINOS Near and Far Detector Readout Systems at a Test Beam, Nucl. Instrum. Meth. A609, 106 (2009).

[96] A. Cabrera, Systematic Comparison of the MINOS Near and Far Detector Readout Systems, PhD thesis U. of Oxford 2005, FERMILAB-THESIS2005-50.

[97] J. A. Formaggio and G. P. Zeller, From eV to EeV: Neutrino Cross Sections Across Energy Scales, Rev. Mod. Phys. 84, 1307 (2012).

[98] MINERvA Collaboration: P. A. Rodrigues et al., Identification of nuclear effects in neutrino-carbon interactions at low three-momentum transfer, Phys. Rev. Lett. 116, 071802 (2016).

[99] J. J. Hartnell, Measurement of the calorimetric energy scale in MINOS, PhD thesis U. of Oxford 2005, FERMILAB-THESIS-2005-51.

[100] N. Tagg et al., Performance of Hamamatsu 64-anode photomultipliers for use with wavelength-shifting optical fibres, Nucl. Instrum. Meth. A539, $668(2005)$.

[101] K. Lang et al., Characterization of 1600 Hamamatsu 16-anode photomultipliers for the MINOS Far detector, Nucl. Instrum. Meth. A545, 852 (2005). 
[102] P. Adamson et al., The MINOS light injection calibration system, Nucl. Instrum. Meth. A492, 325 (2002).

[103] C. B. Smith, Calibration of the MINOS detectors and extraction of neutrino oscillation parameters, $\mathrm{PhD}$ thesis U. Coll. London 2002, FERMILABTHESIS-2002-58.

[104] P. A. Symes, Preliminary Measurement of Neutrino Oscillation Parameters By NuMI/MINOS and Calibration Studies for Improving this Measurement, PhD thesis U. of Sussex 2005, FERMILAB-THESIS-2005-76.

[105] P. Adamson et al., The MINOS calibration detector, Nucl. Instrum. Meth. A556, 119 (2006).

[106] D. Boehnlein et al., Steel Mass and Meltcode Distribution in the MINOS Far Detector, (2004), NuMI-NOTE-STEEL-1061.

[107] M. A. Kordosky, Hadronic interactions in the MINOS detectors, PhD thesis U. of Texas at Austin 2004, FERMILAB-THESIS-2004-34.

[108] P. L. Vahle, Electromagnetic interactions in the MINOS detectors, $\mathrm{PhD}$ thesis U. of Texas at Austin 2004, FERMILAB-THESIS-2004-35.

[109] M. Campanella, A. Ferrari, P. R. Sala, and S. Vanini, First Calorimeter Simulation with the FLUGG Prototype, (1999), ATL-SOFT-99-004.

[110] M. Campanella, A. Ferrari, P. R. Sala, and S. Vanini, Reusing Code from FLUKA and GEANT4 Geometry, (1998), ATL-SOFT-98-039.

[111] GEANT4 Collaboration: S. Agostinelli et al., GEANT4-A Simulation toolkit, Nucl. Instrum. Meth. A506, 250 (2003).

[112] F. Ballarini et al., Nuclear models in FLUKA: Present capabilities, open problems and future improvements, AIP Conf. Proc. 769, 1197 (2005), $[1197(2004)]$. 
[113] R. P. Feynman, Very High-Energy Collisions of Hadrons, Phys. Rev. Lett. 23, 1415 (1969).

[114] Z. Pavlovic, Observation of Disappearance of Muon Neutrinos in the NuMI Beam, PhD thesis U. of Texas at Austin 2008, FERMILAB-THESIS-200859.

[115] M. Dorman, Beam Fit Position Paper, MINOS-doc 7146 (2010).

[116] H. Gallagher, The NEUGEN neutrino event generator, Nucl. Phys. Proc. Suppl. 112, 188 (2002).

[117] C. H. Llewellyn Smith, Neutrino Reactions at Accelerator Energies, Phys. Rept. 3, 261 (1972).

[118] D. Rein and L. M. Sehgal, Coherent pi0 Production in Neutrino Reactions, Nucl. Phys. B223, 29 (1983).

[119] D. Rein and L. M. Sehgal, Neutrino Excitation of Baryon Resonances and Single Pion Production, Annals Phys. 133, 79 (1981).

[120] A. Bodek and U. K. Yang, Modeling deep inelastic cross-sections in the few GeV region, Nucl. Phys. Proc. Suppl. 112, 70 (2002), [,70(2002)].

[121] T. Sjostrand, S. Mrenna, and P. Z. Skands, PYTHIA 6.4 Physics and Manual, JHEP 05, 026 (2006).

[122] Z. Koba, H. B. Nielsen, and P. Olesen, Scaling of multiplicity distributions in high-energy hadron collisions, Nucl. Phys. B40, 317 (1972).

[123] T. Yang, C. Andreopoulos, H. Gallagher, K. Hoffmann, and P. Kehayias, A Hadronization Model for Few-GeV Neutrino Interactions, Eur. Phys. J. C63, 1 (2009). 
[124] R. Merenyi et al., Determination of pion intranuclear rescattering rates in $\nu_{\mu}$-Ne versus $\nu_{\mu}$-D interactions for the atmospheric neutrino flux, Phys. Rev. D45, 743 (1992).

[125] R. Hatcher and S. Kasahara, The (Attempt at a Semi-) Definitive Guide to MINOS Geometry, MINOS-doc 7828 (2010).

[126] C. Zeitnitz and T. A. Gabriel, The GEANT - CALOR interface and benchmark calculations of ZEUS test calorimeters, Nucl. Instrum. Meth. A349, 106 (1994).

[127] J. J. Evans, Measuring Antineutrino Oscillations with the MINOS Experiment, PhD thesis U. of Oxford 2008, FERMILAB-THESIS-2009-14.

[128] R. Frühwirth, Application of Kalman filtering to track and vertex fitting, Nucl. Instrum. Meth. A262, 444 (1987).

[129] J. Marshall, A study of muon neutrino disappearance with the MINOS detectors and the NuMI neutrino beam, PhD thesis Cambridge U. 2008, FERMILAB-THESIS-2008-20.

[130] C. Smith, Beam Fit Position Paper, MINOS-doc 2821 (2007).

[131] C. J. Backhouse, Measuring neutrino oscillation parameters using $\nu_{\mu}$ disappearance in MINOS, PhD thesis U. of Oxford 2011, FERMILAB-THESIS2011-17.

[132] J. Evans, Absolute (calorimetric) shower energy uncertainty, MINOS-doc $7173(2010)$.

[133] T. M. Raufer, A study of neutrino oscillations in MINOS, PhD thesis U. of Oxford 2007, FERMILAB-THESIS-2009-14.

[134] G. Tinti, The ND Preselection for the NC analysis, MINOS-doc 7127 (2010). 
[135] R. Ospanov, A measurement of muon neutrino disappearance with the MINOS detectors and NuMI beam, PhD thesis U. of Texas at Austin 2008, FERMILAB-THESIS-2008-04.

[136] J. S. Ratchford, Identifying Muons for Neutrino Oscillation and Cross Section Experiments, PhD thesis U. of Texas at Austin 2012, FERMILABTHESIS-2012-40.

[137] P. Rodrigues, Notes on the Normalization Systematic, MINOS-doc 6636 (2009).

[138] T. H. Osiecki, A search for sterile neutrinos in MINOS, PhD thesis U. of Texas at Austin 2007, FERMILAB-THESIS-2007-77.

[139] The MINOS Calibration Group in 2009 Collaboration, Position Paper on Calibration of Runs, MINOS-doc 6717 (2010).

[140] M. Kordosky, H. Gallagher, and S. Dytman, Shower Energy Scale Uncertainty for the Run I+II CC Analysis, MINOS-doc 4287 (2008).

[141] G. M. Tinti, Sterile neutrino oscillations in MINOS and hadron production in $p C$ collisions, PhD thesis U. of Oxford 2010, FERMILAB-THESIS-201044.

[142] C. Andreopoulos et al., Updated Cross Section Model Uncertainties for the Charged Current Analysis, MINOS-doc 2989 (2007).

[143] V. Bernard, L. Elouadrhiri, and U.-G. Meissner, Axial structure of the nucleon: Topical Review, J. Phys. G28, R1 (2002).

[144] R. Gran, QE cross-section systematics and MINOS oscillation results, MINOS-doc 2972 (2007). 
[145] MINOS Collaboration: P. Adamson et al., Study of quasielastic scattering using charged-current ?? -iron interactions in the MINOS near detector, Phys. Rev. D91, 012005 (2015).

[146] J. Evans, K. Lang, and R. Nichol, Position paper on the 2010 analysis of charged current events from runs I, II and III, MINOS-doc 7218 (2010).

[147] MINOS Collaboration: P. Adamson et al., Search for sterile neutrino mixing in the MINOS long baseline experiment, Phys. Rev. D81, 052004 (2010).

[148] P. Rodrigues and L. Hsu, Charged current background in the neutral current sample, MINOS-doc 3878 (2007).

[149] J. Long, Position Paper for the Runs I-III FD NC Cleaning, MINOS-doc 7165 (2010).

[150] J. S. Conway, Incorporating Nuisance Parameters in Likelihoods for Multisource Spectra, in Proceedings, PHYSTAT 2011 Workshop on Statistical Issues Related to Discovery Claims in Search Experiments and Unfolding, CERN, Geneva, Switzerland 17-20 January 2011, pages 115-120 2011.

[151] NA49 Collaboration: C. Alt et al., Inclusive production of charged pions in $\mathrm{p}+\mathrm{C}$ collisions at $158 \mathrm{GeV} / \mathrm{c}$ beam momentum, Eur. Phys. J. C49, 897 (2007).

[152] M. Bonesini, A. Marchionni, F. Pietropaolo, and T. Tabarelli de Fatis, On particle production for high-energy neutrino beams, Eur. Phys. J. C20, 13 (2001).

[153] A. Radovic and M. Kordosky, A Position Paper Describing a Hadron Production Uncertainty Covariance Matrix, MINOS-doc 10569 (2014). 
[154] MINER $\nu$ A Collaboration: L. Aliaga et al., Design, Calibration, and Performance of the MINER $\nu$ A Detector, Nucl. Instrum. Meth. A743, 130 (2014).

[155] MINER $\nu$ A Collaboration: L. Aliaga et al., Neutrino Flux Predictions for the NuMI Beam, Submitted to: Phys. Rev. D (2016).

[156] J. Biggs, K. Bourkland, J. Hylen, and M. Kucera, Calibration of NuMI horn current readout, MINOS-doc 1303 (2006).

[157] R. Zwaska et al., Beam-Based Alignment of the NuMI Target Station Components at FNAL, Nucl. Instrum. Meth. A568, 548 (2006).

[158] S. Kopp and L. Loiacono, Proton Beam Quality, MINOS-doc 2854 (2007).

[159] MINOS Collaboration: P. Adamson et al., Search for active neutrino disappearance using neutral-current interactions in the MINOS long-baseline experiment, Phys. Rev. Lett. 101, 221804 (2008).

[160] MINOS Collaboration: P. Adamson et al., Active to sterile neutrino mixing limits from neutral-current interactions in MINOS, Phys. Rev. Lett. 107, 011802 (2011).

[161] A. Palazzo, An estimate of $\theta_{14}$ independent of the reactor antineutrino flux determinations, Phys. Rev. D85, 077301 (2012).

[162] J. Huang, Sterile Neutrino Searches in MINOS/MINOS+Experiment, PhD thesis U. of Texas at Austin 2015, FERMILAB-THESIS-2015-06.

[163] A. V. Devan, Sterile Neutrino Search with MINOS, PhD thesis Coll. William and Mary 2015, FERMILAB-THESIS-2015-12.

[164] Daya Bay Collaboration: F. P. An et al., New Measurement of Antineutrino Oscillation with the Full Detector Configuration at Daya Bay, Phys. Rev. Lett. 115, 111802 (2015). 
[165] RENO Collaboration: S.-H. Seo, New Results from RENO and The $5 \mathrm{MeV}$ Excess, AIP Conf. Proc. 1666, 080002 (2015).

[166] Double Chooz Collaboration: Y. Abe et al., Improved measurements of the neutrino mixing angle $\theta_{13}$ with the Double Chooz detector, JHEP 02, 074 (2015).

[167] G. L. Fogli et al., Global analysis of neutrino masses, mixings and phases: entering the era of leptonic CP violation searches, Phys. Rev. D86, 013012 (2012).

[168] Daya Bay Collaboration: F. P. An et al., Spectral measurement of electron antineutrino oscillation amplitude and frequency at Daya Bay, Phys. Rev. Lett. 112, 061801 (2014).

[169] F. James and M. Roos, Minuit: A System for Function Minimization and Analysis of the Parameter Errors and Correlations, Comput. Phys. Commun. 10, 343 (1975).

[170] G. J. Feldman and R. D. Cousins, A Unified approach to the classical statistical analysis of small signals, Phys. Rev. D57, 3873 (1998).

[171] Super-Kamiokande Collaboration: K. Abe et al., Limits on sterile neutrino mixing using atmospheric neutrinos in Super-Kamiokande, Phys. Rev. D91, $052019(2015)$.

[172] CDHSW Collaboration: F. Dydak et al., A Search for $\nu_{\mu}$ Oscillations in the $\Delta m^{2}$ Range $0.3-90 \mathrm{eV}^{2}$, Phys. Lett. B134, 281 (1984).

[173] CCFR Collaboration: I. E. Stockdale et al., Limits on $\nu_{\mu}$ Oscillations in the Mass Range $30<\Delta m^{2}<1000 \mathrm{eV}^{2}$, Phys. Rev. Lett. 52, 1384 (1984). 
[174] SciBooNE, MiniBooNE Collaboration: K. B. M. Mahn et al., Dual baseline search for muon neutrino disappearance at $0.5 \mathrm{eV}^{2}<\Delta m^{2}<40 \mathrm{eV}^{2}$, Phys. Rev. D85, 032007 (2012).

[175] M. Abbes et al., The Bugey-3 neutrino detector, Nucl. Instrum. Meth. A374, 164 (1996).

[176] Y. Declais et al., Search for neutrino oscillations at 15, 40, and 95 metres from a nuclear power reactor at Bugey, Nucl. Phys. B434, 503 (1995).

[177] OPERA Collaboration: N. Agafonova et al., Search for $\nu_{\mu} \rightarrow \nu_{e}$ oscillations with the OPERA experiment in the CNGS beam, JHEP 07, 004 (2013), [Addendum: JHEP07,085(2013)].

[178] NOMAD Collaboration: P. Astier et al., Search for $\nu_{\mu} \rightarrow \nu_{e}$ oscillations in the NOMAD experiment, Phys. Lett. B570, 19 (2003).

[179] P. Huber, Erratum: determination of anti-neutrino spectra from nuclear reactors, Phys. Rev. C85, 029901 (2012).

[180] J. Neyman, Outline of a Theory of Statistical Estimation Based on the Classical Theory of Probability, Trans. Cambridge Phil. Soc A236, 333 (1937).

[181] W. Feller, The fundamental limit theorems in probability, Bull. Amer. Math. Soc. 51, 800 (1945).

[182] F. James, Statistical methods in experimental physics (World Scientific, 2006).

[183] S. S. Wilks, The Large-Sample Distribution of the Likelihood Ratio for Testing Composite Hypotheses, Annals Math. Statist. 9, 60 (1938). 
[184] SciBooNE, MiniBooNE Collaboration: G. Cheng et al., Dual baseline search for muon antineutrino disappearance at $0.1 \mathrm{eV}^{2}<\Delta m^{2}<100 \mathrm{eV}^{2}$, Phys. Rev. D86, 052009 (2012).

[185] CCFR Collaboration: I. E. Stockdale et al., Search for $\nu_{\mu}$ and $\bar{\nu}_{\mu}$ oscillations in the mass range $15<\Delta m^{2}<1000 \mathrm{eV}^{2}$, Z. Phys. C27, 53 (1985). 